\title{
Sound of the Drum, Energy of the Dance - Making the Lake Huron Treaty Atlas the Anishinaabe Way
}

by

Stephanie Anne Pyne

A thesis submitted to the Faculty of Graduate and Postdoctoral Affairs in partial fulfillment of the requirements for the degree of

Doctor of Philosophy

in

Geography with Specialization in Political Economy

Carleton University

Ottawa, Ontario

(C2013 Stephanie Pyne 


\begin{abstract}
This thesis takes a reflexive narrative approach to critically interpreting the iterative processes involved in the making of the Lake Huron Treaty Atlas (the Atlas). The Atlas is an interactive, multimedia, geospatial web product that reflects an inclusive approach to telling the story of the Robinson Huron Treaty relationship process over time and across space, bringing together a variety of historical and geographical perspectives. Both the thesis and the Atlas interpret Anishinaabe perspectives and incorporate them in their style and approach; they participate in the current trend in critical cartography to engage in mapping in new ways by reflecting the multiple dimensions of socioeconomic, political and cultural 'reality'. Making contributions in many areas, including in the conceptual and practical spheres of cartography, this thesis comments on some deep trends such as the spatial turn to performance and the critical turn to interpretation. In addition, it participates in the seventh fire project of reconciliation reflected in the significant Anishinaabe Teaching, the Seven Fires Prophecy, by promoting a holistic and emergent approach to development, and by emphasizing the possibility of bridging perspectives to build intercultural awareness through the collaborative creation of the Atlas as a reconciliation tool.

This thesis asserts that through holistic, reflexive and critical cartographic practice, it is possible not only to acknowledge Anishinaabe perspectives in mapping processes, but to integrate them as well, in a manner that (1) preserves their inherent meaning and value, and (2) augments the meaning of the cybercartographic mapping processes. I aim to demonstrate how this type of practice has been employed in the Lake Huron Treaty Atlas project by providing
\end{abstract}


critically reflexive narrative accounts of the Travels in the Making of the Atlas Map and the Map of Maps, each of which functions as a narrative portal to the other maps in the Atlas. 


\section{Acknowledgements}

Chi-Miigwetch first and foremost to the Ancestors, who are many and who come from many places; to the Rock People, who have helped and guided me to come to know who I am; who inspired me, supported me, guided me, and danced with me - who have urged me to tell this story.

My first thoughts on writing this Acknowledgements section were to include the names of all of the Lake Huron Treaty Atlas contributors together with a brief summary of their contributions - that was, until I realized that would practically comprise an entire thesis length and tire readers out before they reached the Table of Contents! Instead, I extend my most heartfelt gratitude to all those mentioned in this thesis, which also describes your contributions to various extents; in addition to the many people I have not had the opportunity to mention. To these people, and to those mentioned in the thesis, I can say that I intend to write another piece once this thesis is completed that provides further details and stories concerning the involvement and contributions of everyone I can possibly remember. This piece will present a broad view of "community" and illuminate the reality of the (Algonquin) Anishinaabemowin phrase “Dawnoweh-ma-geneduc (All My Relatives)". In addition, excerpts from this future writing and associated media will be uploaded to the various maps in the Lake Huron Treaty Atlas.

I am grateful that the work to produce the working Lake Huron Treaty Atlas and to complete this thesis were generously supported first, in phase I, by an Inukshuk Grant and second, in phases II and III, by a SSHRC Standard Research Grant and a SSHRC Outreach Grant, respectively. I am also grateful for the 
ongoing financial and related support of Carleton University, in particular the Department of Geography and Environmental Studies over the past seven years.

Special acknowledgement and thanks to the Late Dr. William Commanda for his Teachings, spiritual support and prayers for the Lake Huron Treaty Atlas (and thesis) project; to the Late Elder Blaine Belleau for sharing his unique, inspiring and challenging insights with me over the years; to my good friend the Late Murray Stephens whose transcriptions of the Dennis Field Notes remain on the agenda to be mapped one day; to the Late Grandmother Louise Wawatie who inspired and encouraged me with her gift for visioning and her living commitment to healing and the land; and finally, to the Late Shirley Firth, whose lasting impact I only realized during the writing of this thesis - all of whom passed during the course of this thesis project. The rate at which the Elders are passing makes now the time to seek to understand their critical insights.

From time to time over the past seven years, I have estimated the number of people I have engaged with in discussions and collaborations related to this thesis and various aspects of the atlas development process. Each year, the number of people grows, as does awareness of the Lake Huron Treaty in a broad historical and geographical context. For this, I am happy and thankful to each person who took the time to listen, share their views, and contribute in other ways.

Apart from thanking my supervisor Dr. Fraser Taylor for being the most ideal doctoral supervisor and atlas project principal investigator that I could imagine, I thank my family, which extends to all of my relations. 


\section{Contents}

$\begin{array}{ll}\text { Abstract } & \text { ii }\end{array}$

Acknowledgements $\quad$ iv

List of Tables $\quad x$

List of Figures $\quad$ xi

List of Appendices $\quad$ xvii

$\begin{array}{ll}\text { Foreword } & \text { xviii }\end{array}$

$\begin{array}{ll}\text { Preface } & \text { xxvii }\end{array}$

I Atlas Development in a Reconciliation Context: 1 Introduction

1 Reconciliation in a Seven Fires Prophecy Context 11

1.1 The Seven Fires Prophecy and the Wampum Belt 14

1.2 Reconciliation in a Seven Fires Prophecy Context 16

1.3 The Seven Fires Prophecy, Reconciliation and the 25

Lake Huron Treaty (Atlas) - Concluding Remarks

2 Reconciliation and Development as Bimaadiziwin 26

2.1 The Call to Spatialize History in the Name of Reconciliation 27

$2.2 \quad$ Working Toward a Holistic Approach to Development 31

2.3 Applying a Holistic Approach to Development as Bimaadiziwin 43 in the Making of the Lake Huron Treaty Atlas - Concluding Remarks

$3 \quad$ Crystallization in the Seventh Moment: Thesis Method 47

3.1 Qualitative Inquiry and Crystallization 49

3.2 Reflexive Methods 51

3.3 Critical Interpretive Method 54

3.4 Reflexive Narrative Analysis 55

3.5 Critical Conceptual Analysis 57

3.6 The Reflexive Relationship between the Thesis and the Atlas 57

3.7 Reflexivity and the Two-pronged approach to the Lake Huron Treaty 59 Atlas - Concluding Remarks 


\section{Re-visioning Cartography in A Growing Community}

$4 \quad$ The Spatial Turn to Performance: Critical Perspectives in Cartography

4.1 Cartography as Deconstruction

4.2 The Spatial Turn to Performance in Critical Cartography

4.3 Cartography and Community

4.4 Maps as Narratives of Experience $\quad 79$

4.5 Critical Cartography and Policy - Concluding Remarks 80

$5 \quad$ Adding Voice to Mapped Silences: Critical Indigenous Cartographies

5.1 The Land as Map

5.2 Early Colonial Mapping Encounters 90

5.3 Mapping with the Newcomers in a Reconciliation Context 92

5.4 Links to Critical Cartography - Concluding Remarks 97

\section{Developing an Atlas for Development 98}

6 Cybercartography: Growing an Atlas Vision in Good Soil 99

6.1 Defining the Map and Cartography 100

6.2 Why an Atlas? Why not just a Map? 102

6.3 Cybercartographic Atlas Design and Development 104

6.4 The Cyber in Cybercartography 110

6.5 Emerging Development and the Lake Huron Treaty Atlas -- Charting 110 New Territory in New Ways using the Cybercartographic Approach

6.6 The Lake Huron Treaty Atlas as a Bridging Tool - Concluding Remarks 117

$7 \quad$ Tracing the Iterative Dimensions of the Lake Huron Treaty 118 Atlas Process

7.1 The "Stuff" of Iterative Processes: Talk, Templates and Tradition 119

7.2 Iterative Processes in the Design and Development of the 121 Lake Huron Treaty Atlas

7.3 Multiple Dimensions: Inter-dimensional Iterative Processes 123

$\begin{array}{ll}7.4 & \text { Collaborative Relationships } \\ 7.5 & 126\end{array}$

$\begin{array}{ll}7.5 & \text { Iterative Processes and Geonarrative } \\ 7.61\end{array}$

7.6 Iterative Processes and the Anishinaabe Perspective - 132

IV Waasaa-inaabidaa: We Look In All Directions 134 
8 Reflexive Tracking of the Travels in the Making of the Atlas Map

8.1 The Story of How I Became the Treaties Module Research Assistant 137

8.2 Getting my Feet Wet - Swimming in the Seas of Cybercartography ... 140 and History

8.3 Land in Sight - Lake Huron Treaty Region Aho(y) 143

8.4 Post-epiphany Focus 144

8.5 Story Circle - Patricia Kennedy and the Governance Paper Trail Map 145

8.6 The 43rd Algonkian Conference, Toronto, Ontario, October 18-20, 2007146

8.7 Reaching Out - Early Collaborator Contacts 147

8.8 An Unexpected Mapping Epiphany 148

8.9 First Visit to the Lake Huron Treaty Region - 151 An Adopted Homecoming

8.10 Work at the Lab - Winter $2008 \quad 168$

8.11 The "Visiting the Circle - Enhancing the Relationship" 169 Conference, Parry Sound, March 24, 2007

8.12 Work at the Lab - Pre-launch "Festivities" 172

8.13 Community Launch at Shawanosowe School, April 23, $2008 \quad 173$

8.14 The Ottawa launch of the fledgling Atlas of Indigenous 176 Perspectives and Knowledge

$\begin{array}{lll}8.15 & \text { Post-launch Requirements } & 179\end{array}$

8.16 Travels, Work at the Lab and Funding Proposal Preparation: 181 Spring - Summer 2008

$9 \quad$ Navigating the Narrative Logic of the Map of Maps 184

9.1 The Map of Maps 185

9.2 The Travels in the Making of the Atlas Map 187

$\begin{array}{lll}9.3 & \text { The Treaties Module } & 189\end{array}$

9.4 The Survey Journeys Map 191

9.5 The Nations not Surveyed between 1851 and 1853 Map 196

9.6 The Marlatt Commentaries Map 198

9.7 The Treaties, Agreements and Proposals Map 199

9.8 The Bagone-giizhig (Hole in the Day) Biography Map 200

9.9 The J.W. Keating Biography Map 203

9.10 The J.S. Dennis Biography Map 205

9.11 The History According to Blaine Belleau Map 207

9.12 The War of 1812 Map 209

9.13 The Vidal Anderson Investigative Commission of 1849 Map 211

9.14 The Governance Paper Trail Map 213

$\begin{array}{ll}9.15 & \text { The Gathering Places Map } \\ 9.16 & 215\end{array}$

9.16 The Community Stories Map 216

9.17 The Residential Schools Map 217

9.18 The E.F. Wilson Biography Map 219

9.19 The Carl Beam Map 221

9.20 The Nanboozhoo Mindemoye Story Map 223

9.21 The Language and Culture Map 224 
9.22 The Current Affairs in the Lake Huron Region Map 225

9.23 The Treaty and Consultation Current Affairs Map 226

9.24 Works in Progress - Concluding Remarks 227

V Dancing into the Future - Building Awareness to Bridge Relationships

10. Intercultural Bridging - Iterative Development and 230 Emergent Mapping Practices

10.1 Reflexivity 230

10.2 Performance 231

$\begin{array}{lll}10.3 & \text { Relational Space } & 232\end{array}$

$\begin{array}{lll}10.4 & \text { Narrative } & 233\end{array}$

10.5 Inclusivity and Experience $\quad 234$

10.6 Bridging the Gap - Concluding Remarks 239

11. Atlas Development Reflecting the Past, Reconciling the Future 241

11.1 Future Implementation - Potential and Challenges 244

$\begin{array}{lll}11.2 & \text { Turtle Speed } & 245\end{array}$

11.3 Shifting the Research Focus 246

11.4 Benefits of Atlas Framework for Research 247

11.5 The Lake Huron Treaty Atlas and Development 248

11.6 The Lake Huron Treaty Atlas and the Seven Fires Prophecy: 250 A Map Proposal

11.7 Energy of the Dance and Performativity - Concluding Remarks 255

Afterword: Initial Reflections on the 259 Thesis-writing Process

$\begin{array}{ll}\text { Bibliography } & 263\end{array}$

$\begin{array}{ll}\text { Appendices } & 286\end{array}$

Appendix A Full Text of the Seven Fires Prophecy 287

Appendix B List of Presentations 290

Appendix C Phase II and Phase III Selected Fieldwork Summary 292

Appendix D Selected Media 307

Appendix E Thesis-related Publications 309 


\section{List of Tables}

10.1 Table 10.1. Common characteristics of epiphanies 237 (McDonald, 2008), and two Lake Huron Treaty Atlas epiphanies. 


\section{List of Figures}

F.1 Screenshot showing first pane of Spirit Sub-module in $\quad$ xxi Treaties Module of the Atlas of Indigenous Perspectives and Knowledge.

F.2 Screenshot showing second pane of Spirit Sub-module in xxii Treaties Module of the Atlas of Indigenous Perspectives and Knowledge.

F.3 Screenshot showing third pane of Spirit Sub-module in xxiii Treaties Module of the Atlas of Indigenous Perspectives and Knowledge.

F.4 Screenshot showing fourth pane of Spirit Sub-module in xxiv Treaties Module of the Atlas of Indigenous Perspectives and Knowledge.

F.5 Screenshot showing fifth pane of Spirit Sub-module in $\quad x x v$ Treaties Module of the Atlas of Indigenous Perspectives and Knowledge.

1.1 William Commanda with the Seven Fires Prophecy Belt (Prayer Vigil for the Earth website, http://oneprayer4.zenfolio.com/?q=commanda).

2.1 The Atlas Drum - Hand drum made for me by Isabelle Meawasige and Blain Commanda (both from Serpent River First Nation) and hand painted by Stan Panamick (from $M^{\prime}$ Chigeeng First Nation.

4.1 Screenshot showing 1849 Settlement Map in the Treaties Module of the Atlas of Indigenous Perspectives and Knowledge as background for Surveyor Dennis's journey out of " organized territory", and into "unorganized" unsettled Anishinaabe Territories; illustrating both deconstructive and constructive - reflexive - approaches to critical cartography.

5.1 Screenshot showing Jacob Wawatie teaching through the Turtle Island and the Elephant map story, which touches on many dimensions of existence on Mother Earth.

8.1 Looking out at Lake Nipissing with Daniel Gibson, morning, February 15, 2008 (photo by Stephanie Pyne). 
8.2 Photo from Dokis: Since Time Immemorial (2006) taken many years ago of the dock at Dokis First Nation.

8.3 One branch of the French River on Dokis First Nation territory, 155 afternoon, February 15, 2008 (photo by Stephanie Pyne).

8.4 View of lake from Atikameksheng Anishnawbek Roadside, afternoon, February 16, 2008 (photo by Stephanie Pyne).

8.5 Entering $\mathrm{M}^{\prime}$ Chigeeng First Nation Territory, morning, February 18, 2013 (photo by Stephanie Pyne).

8.6 Initial conceptual design diagram for the Treaties Module allowing for potential expansion into an Atlas.

8.7 Whitefish Falls, one location that was, but no longer is, included within Wigwaasminising (Whitefish River, Reserve No. 4) surveyed boundaries, morning, February 19, 2008 (photo by Stephanie Pyne).

8.8 Stephanie Pyne in front of Shawanosowe School, February 19, 2008 (photo by Gimaah Shining Turtle).

8.9 The new site of the Bell Rocks after the La Farge company removed them from the flat rock where they once stood, morning, February 19, 2008 (photo by Stephanie Pyne).

8.10 Screenshot showing point for Bawating, September 5-9, 1850, 166 during the Robinson Treaties signing ceremonies in the Marlatt Commentaries Map of the Lake Huron Treaties Atlas.

8.11 According to the late Blaine Belleau, Surveyor Dennis likely stood on this small mountain to make one of his readings, afternoon, February 20, 2008 (photo by Stephanie Pyne).

8.12 North Channel Lake Huron, afternoon, February 21, 2008 (photo by Stephanie Pyne).

8.13 Driving through Algonquin Park on the way to the 169 conference in Parry Sound, afternoon, March 23, 2008 (photo by Jesse Young). 
8.14 Driving through Algonquin Park on the way to the

conference, Parry Sound, evening, March 23, 2008

(photo by Jesse Young).

8.15 Sample of Bill Allen's photography. This image is included

171 in the Survey Journeys Maps. Looking toward Sandy Island (Isles aux Sables), the island that was originally referred to in the Robinson Huron Treaty reserve description for Wausauksing First Nation (photo by Bill Allen).

8.16 Looking south toward Wigwaasminising, afternoon, April 23, 2008 (photo by Stephanie Pyne).

8.17 Looking north toward the LaCloche Mountains from Wigwaasminising, afternoon, April 23, 2008 (photo by Stephanie Pyne).

8.18 Whitefish Falls, afternoon, April 23, 2008 (photo by Stephanie Pyne).

8.19 Screenshot showing first pane of Survey Journeys Maps in the Treaties Module.

8.20 Group photo of some Atlas contributors and launch guests, evening, April 23, 2008. Front: Late Dr. William Commanda Second Row (left to right: Stephanie Pyne, Gimaah Wilmer Noganosh, Magnetawan First Nation, the Late Blaine Belleau (Gitigaan-ziibing or Garden River First Nation), Brad Henry and Christina Moore (People of the Longhouse).

Back Row: Bill Allen (photo by Alan Corbiere).

8.21 Group photo of some Atlas contributors and launch guests, evening, April 23, 2008. Left to right: Stephanie Pyne, Gimaah Wilmer Noganosh, Magnetawan First Nation, the Late Blaine Belleau (Gitigaan-ziibing Garden River First Nation), Amos Hayes, and Alan Corbiere (photo by Bill Allen).

8.22 Atlas demonstrations and discussions (photo by Alan Corbiere).

9.1 Screenshot of the Map of Maps showing points for all of the 185 maps in the Lake Huron Treaty Atlas.

9.2 Screenshot of Travels in the Making of the Atlas Map showing 
some of the stops in my atlas-making travels; with the point for Manitowaning highlighted and the associated media and media descriptions visible along with the pop-up window displaying different map viewing options.

9.3 Screenshot showing first pane of Treaties Module of the Atlas of Indigenous Perspectives and Knowledge.

9.4 Screenshot showing Season Two, In the Field of the Survey Journeys Maps set against Vidal-Anderson Commission, 1849 proposed reserves sketch map and mining lots (in black) surveyed prior to the Robinson Huron Treaty signing.

9.5 Screenshot showing the Season One, Enroute Map with 193 Bridgland's travel stops set against Commercial Map of Canada, 1834.

9.6 Screenshot showing the 'Season Two', 'In the Field' Map with pale green dots representing Bristow's camp stops and yellow dots representing Dennis' camp stops.

9.7 Screenshot showing the point for Atikameksheng Anishinawbek highlighted in blue and the video clip of Gimaah Art Petahtegoose talking about the consequences for his community of not surveying the reserve.

9.8 Screenshot showing point for the Robinson Huron Treaty Signing Ceremony at Bawating (Sault Ste. Marie), September 9, 1850, with video clip of the Late Blaine Belleau discussing the difference between "surrender" and "share".

9.9 Screenshot highlighting the point in blue for the Treaty of Niagara, 1764, signing ceremony at Niagara Falls, with a video clip of Gimaah Isadore Day (Wiindawtegowinini) speaking about the significance of this treaty for future agreements between the Anishinaabek and the Crown, including the Bondhead Treaty; and with Alan Corbiere (mentioned above), walking in the foreground.

9.10 Screenshot showing the point for June 12-16, 1820 at Sault Ste. Marie, Michigan where Bagone-giizhig Sr. helped prevent the British from crossing onto the American side.

9.11 Screenshot highlighting point for Bkejwanong (where the 
waters divide) or Walpole Island, where Keating was an Indian Agent.

9.12 Screenshot highlighting the location of Dennis' childhood home in Weston (now part of Toronto). The home, which is featured in the pop-up image, was later sold and converted into a sanatorium.

9.13 Screenshot showing the scope of the History According to Blaine Belleau Map, with a pop-up image of the Late Blaine Belleau speaking about the mythical foundations of the 1493 Bull Inter Caetera by Pope Alexander VI and the Doctrine of Discovery.

9.14 Screenshot showing sites of some key battles, skirmishes and events in the War of 1812.

9.15 Screenshot showing some of the stops Commissioner Vidal made on his way to the Commission meetings in the Lake Superior region.

9.16 Screenshot showing some of the stops Commissioner Vidal made on his way to the Commission meetings in the Lake Superior region, with a pop-up image of the Vidal-Anderson Commission frame in the Background section of the Treaties Module.

9.17 Screenshot showing most of the points on the Governance Paper Trail Map.

9.18 Screenshot highlighting the point in blue for Bawating, a traditional gathering place with a long history; with pop-up image of Mouth of the Gitigaan-ziibing River (near Bawating).

9.19 Screenshot highlighting point for the Bell Rocks with audio recordings of meetings with the Late Arthur J. (Archie) McGregor and voice-recorded translations by Perry Bebamash reading from Archie's "Sinmedwe'ek", showing pop-up window for Archie telling a story about the Bell Rocks.

9.20 Screenshot showing most of the points on the Residential Schools Map for North America, highlighting the point for Spanish Residential School, which - although in the Lake Huron Treaty region - was attended by children as far east 
as Quebec.

9.21 Screenshot showing point for Kitiganzibi (Garden River) in blue, with pop-up image of digital scan from E.F. Wilson's Journal, in addition to points for many of the places E.F. Wilson lived and visited.

9.22 Screenshot showing points for places lived and travelled by Carl and (later) Ann Beam, with pop-up image of Carl as a young boy with his mother. Based on biographical essay by Ann Beam.

9.23 Screenshot showing two stops in the journey of Nanboozho 223 when he threw his grandmother into the lake and she became Mindemoye Island.

9.24 Screenshot showing the point in blue for the location of 224 Miioodigaazhiwebak (Alligator Rock). Photo by Alan Corbiere.

9.25 Screenshot showing points for news and current affairs in, 225 around and about the Lake Huron Region.

9.26 Screenshot showing broad scope of interrelated treaty and 226 consultation related current affairs.

11.1. Lisa Odjig performing the hoop dance at the Wikwemikong 256 First Nation 2012 Pow Wow. 


\section{List of Appendices}

Appendix A Full Text of the Seven Fires Prophecy 286

Appendix B List of Presentations 289

Appendix C Phase II and Phase III -- Selected Fieldwork Summary 291

Appendix D Selected Digital Media Samples Referenced in Thesis 306

$\begin{array}{ll}\text { Appendix E Thesis-related Publications } & 309\end{array}$ 


\section{Foreword}

Boozhoo. Gaa-bge-bagoonse Kwe ndizhinikaaz. Giwaydin ndojeba. Chi Migizi dodem.

Boozhoo is a traditional Anishinaabe greeting and means, "have you seen him", referring to the Trickster, Nenboozhoo. My Anishinaabe Spirit name is Gaa-bge-bagoonse Kwe, which means Little Prince's Pine Woman; it is essentially the same name given to me in English when I was born. My clan is Gichi-migizi, the Great Eagle; and, I am from the North where I first became aware.

I come from a variety of long lines of people of the land. My ancestry is pervaded by the theme of people from different cultures working together and loving each other through family connections. My Anishinaabe ties were once traced through my paternal Grandmother's lineage to the north shore of the St. Lawrence, west of Montreal where she married her French Huguenot husband. I am a multicultural person with many roots. My father's father came From China to North America with his brother in 1910 when he was a young man. On my mother's side, I have Scottish, Irish and English roots, in addition to possible Roma heritage. Variations of such multicultural heritage are not uncommon in the histories of many people on Turtle Island, something that John Ralston Saul pointed out in A Fair Country: Telling Truths about Canada (2008).

As I have grown - especially since I began university in 1984 - my work has been in the area of healing Western approaches to knowledge that are inconsistent with Whole ways of being. Part of my motivation lies in my awareness of and connection to my Indigenous roots, not only on Turtle Island, 
but on the Elephant (Eurasia, for the most part, Jacob Wawatie (Mowegun or Wolf), personal communication, May 24, 2009) as well.1

The reference to "the sound of the drum" is inspired by the story of the drum told to me by Algonquin Anishinaabe Elder, Jacob Wawatie (Mowegun) from Kokomville, in the Quebec region of Anishinaabe Territory2: In the beginning, there was silence and darkness. With creation came the first sound, the sound of the drum, the heartbeat. To this day, when the Anishinaabek gather for ceremonies from powwows to traditional gatherings, the drum is present, bringing with it the presence of that long distant past and all the times since unified by the drumbeat, the heartbeat. It is the same for the atlas project I have been working on, which is inscribed with my heartbeat. It is with an attitude of care and conscientiousness that I have approached this project; one way I have done this is to integrate it with my life, something that has seemed to flow naturally anyway.

The phrase "energy of the dance" was inspired after a recent drive I took to Mnidoo Mnising, when "the Atlas is a hoop dance" came into my mind as I

${ }^{1}$ The story of Turtle Island (North America) and the Elephant (Eurasia, the body; and, South America, the head) was told to Jacob Wawatie by his Grandmother, Dr. Kokom Lena Jerome Nottaway, and by other Elders; it is intergenerational knowledge that goes back a long way. According to the story, the inhabitants of the Elephant "cut off the head of their God", with the head becoming South America. There are many dimensions to this story, including physical (topographical), spiritual, ecological, historical, social and economic dimensions.

2 Jacob Wawatie (Mowegun) is the founder and director of Kokomville Academy; a knowledgesharing institute committed to combining theory and practice and to integrating Anishinaabe with Western approaches to knowledge. First acknowledged as an Elder at the age of 28 for his knowledge of Anishinaabe culture and language, Jacob has been a teacher since the age of 16 . Through his studies of linguistics at McGill University, Jacob developed a writing system for the Algonquin language, which he later applied in his teaching. With a special interest in curriculum development, he has participated in the design of a number of learning programs geared to integrating the traditional Anishinaabe approaches to education with western approaches. In 1984 , Jacob took what turned out to be a 12-year sabbatical from his position as director of the Rapid Lake School to begin living on the land and learning the traditional knowledge and education system from his grandmother, Dr. Kokom Lena Jerome Nottaway, and from other Elders. Lena was raised living on the land with little usage of European resources, and received an honorary doctorate from Carleton University (Ottawa, Canada) in 1992 for her knowledge of traditional law, language, and culture. 
took a detour before arriving at my first destination to drive through Sheguiandah First Nation, the place where some of the oldest ancestral remains have been located. The significance of this phrase is rooted in its link to "performance", as this thesis will elaborate on.

Finally, the phrase "Making the Lake Huron Treaty Atlas the Anishinaabe Way" refers to the many different ways particular Anishinaabe individuals were involved in the project. It also refers to the many ways I was influenced by my interactions with these individuals, and how this translated into my own Anishinaabe ways.

I begin with a story that came to me in the Niitsitapi ${ }^{3}$ way in September 2004 when I was living in Banff, Alberta: all at once, with complete energy, form and Life, in answer to questions I was pursuing in my master's studies in philosophy. The stories behind that story will be unfolded as this thesis progresses. For now, it is important to say that the Elder I told that story to, Dave Melting Tallow, requested that I include it as the beginning of my Master's thesis. However, it was not the right time or place for that then. Maybe I still needed time to sit, learn, move and grow with the story.

In May 2008, however, I found a place for the story, "Rainbow Raven and the Fly in the Bottle", in the Spirit section of the Treaties Module of the Living Cybercartographic Atlas of Indigenous Perspectives and Knowledge, where it continues to live today as the original spirit, intent and method of the atlas project. Although I have included the text of the story below, I encourage readers to interact with it online instead by clicking on the link in footnote number 4 .

3 "Niitsitapi" is the Blackfoot word for "real people" (Thomas Manyguns, personal communication, December 11, 2004). 


\section{Rainbow Raven and the Fly in the Bottle ${ }^{4}$}

The philosopher's job is to show the fly the way out of the fly bottle. (adapted from Ludwig Wittgenstein, 1953, 2009).5

\section{The Fly}

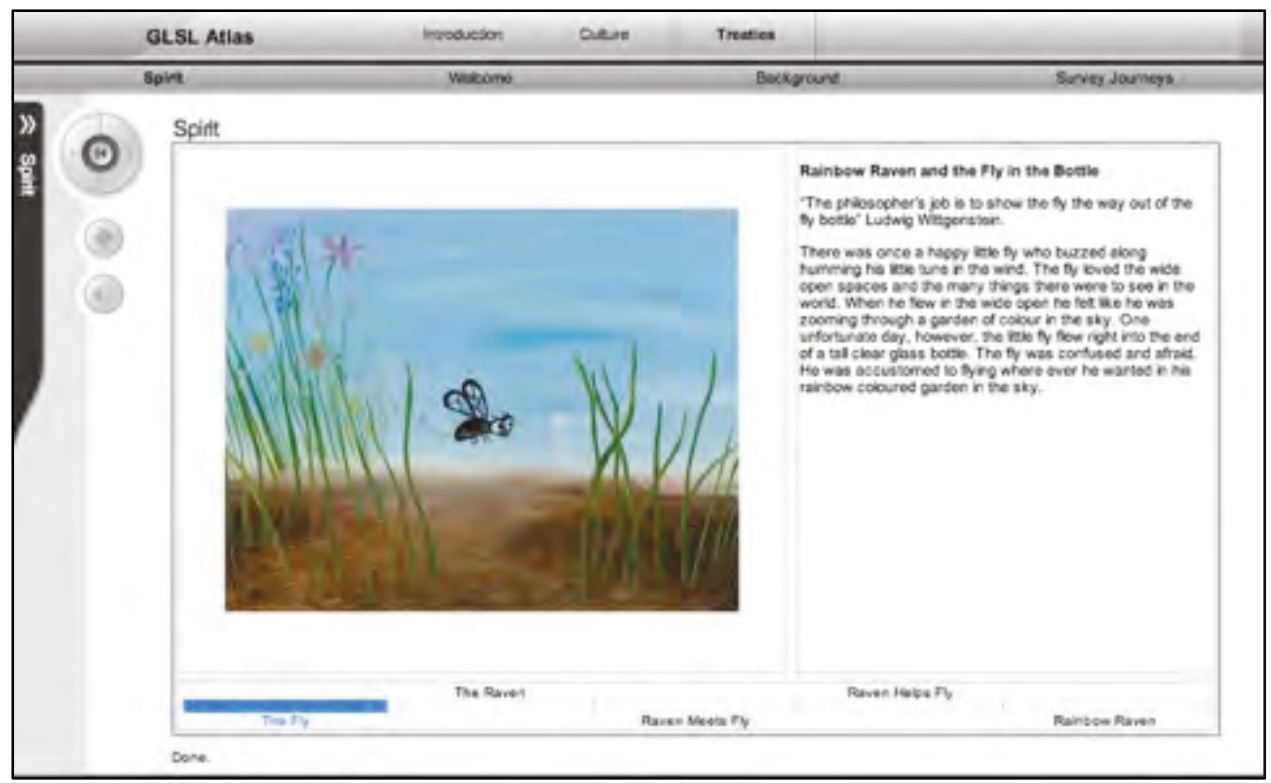

Figure F1. Screenshot showing first pane of Spirit Sub-module in Treaties Module of the Atlas of Indigenous Perspectives and Knowledge.

There was once a happy little fly who buzzed along humming his little tune in the wind. The fly loved the wide open spaces and the many things there were to see in the world. When he flew in the wide open he felt like he was zooming through a garden of colour in the sky. One unfortunate day, however, the little fly flew right into the end of a tall clear glass bottle. The fly was confused and afraid. He was accustomed to flying where ever he wanted in his rainbow coloured garden in the sky.

4 It is best to view the story in either the Safari or Chrome browser at http:/ / atlas.gcrc.carleton.ca/glsl/treaties/treaties_spirit.xml.html - Treaties_Spirit

5 The exact quotation is: " 309 . What is your aim in philosophy? To show the fly the way out of the fly-bottle" $(1953,2009,110)$. 


\section{The Raven}

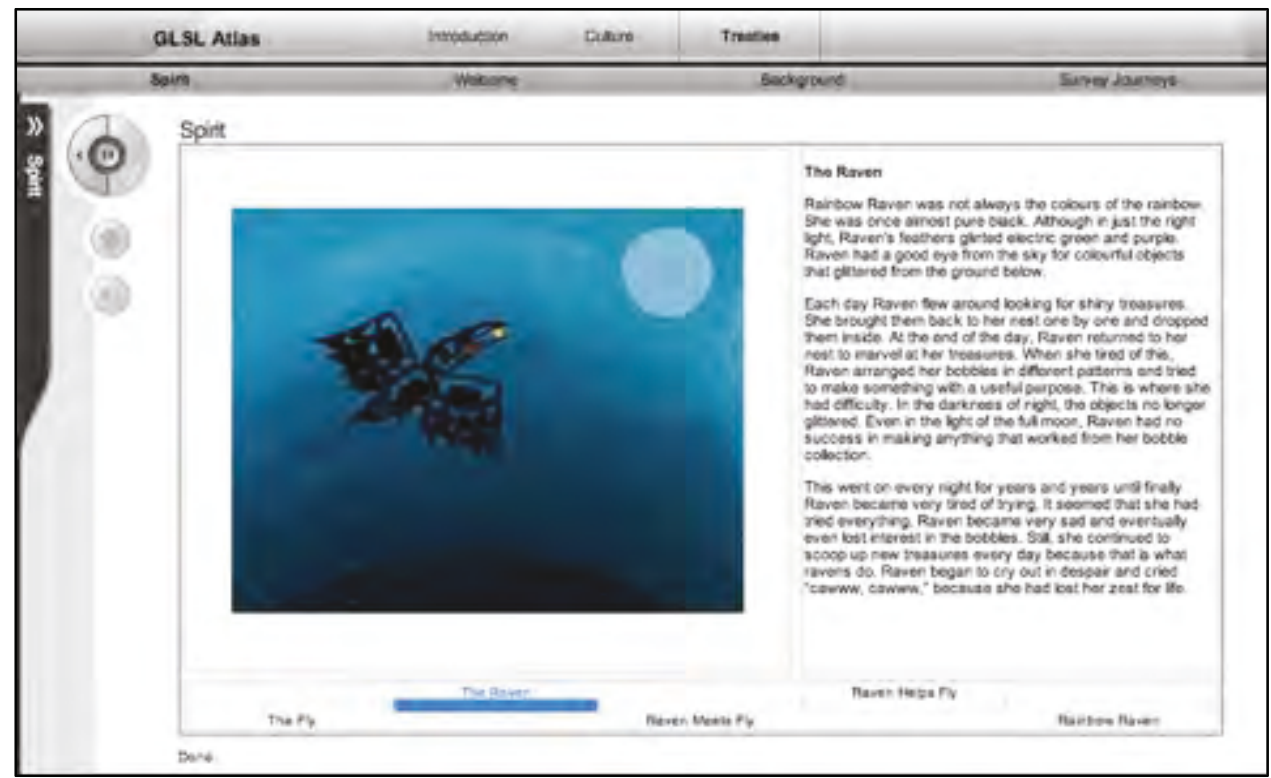

Figure F2. Screenshot showing second pane of Spirit Sub-module in Treaties Module of the Atlas of Indigenous Perspectives and Knowledge.

Rainbow Raven was not always the colours of the rainbow. She was once almost pure black. Although in just the right light, Raven's feathers glinted electric green and purple. Raven had a good eye from the sky for colorful objects that glittered from the ground below.

Each day Raven flew around looking for shiny treasures. She brought them back to her nest one by one and dropped them inside. At the end of the day, Raven returned to her nest to marvel at her treasures. When she tired of this, Raven arranged her bobbles in different patterns and tried to make something with a useful purpose. This is where she had difficulty. In the darkness of night, the objects no longer glittered. Even in the light of the full moon, Raven had no success in making anything that worked from her bobble collection.

This went on every night for years and years until finally Raven became very tired of trying. It seemed that she had tried everything. Raven became very 
sad and eventually even lost interest in the bobbles. Still, she continued to scoop up new treasures every day because that is what ravens do. Raven began to cry out in despair and cried "cawww, cawww", because she had lost her zest for life.

\section{Raven Meets Fly}

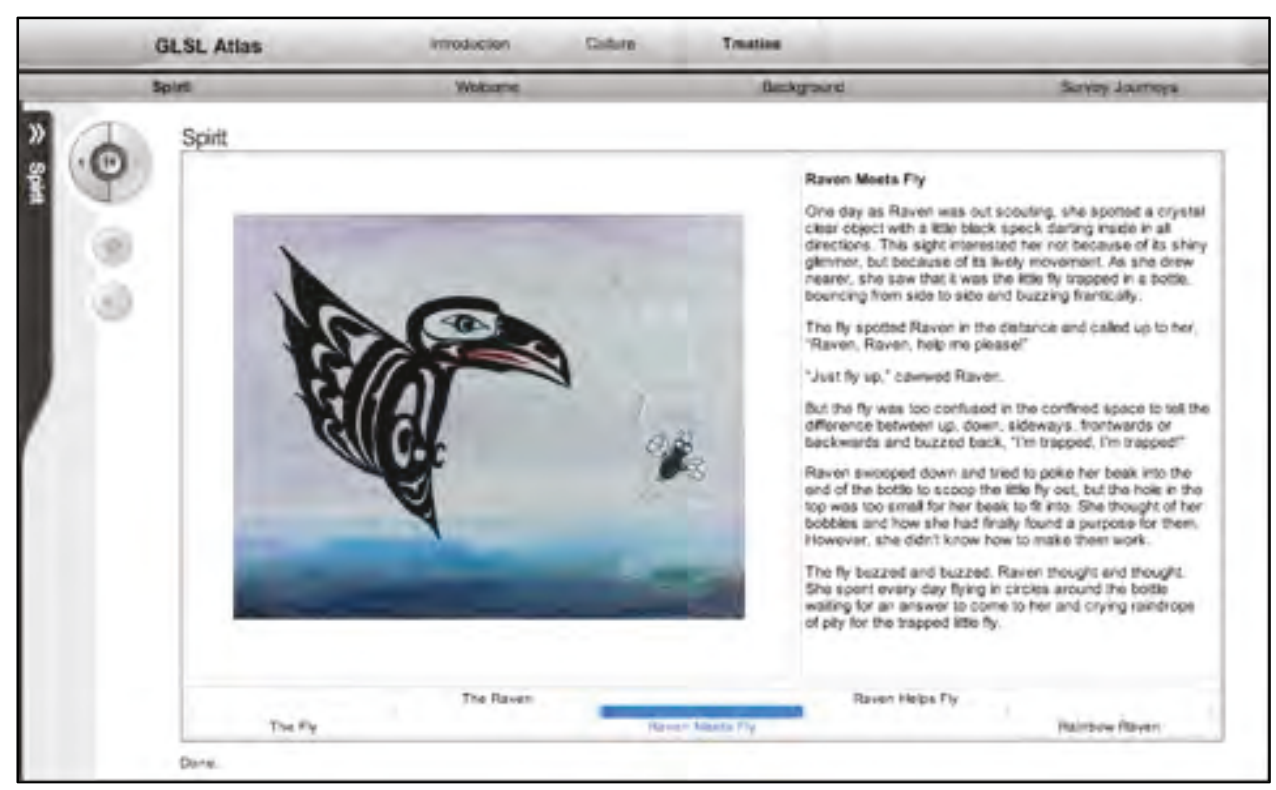

Figure F3. Screenshot showing third pane of Spirit sub-module in Treaties Module of the Atlas of Indigenous Perspectives and Knowledge.

One day as Raven was out scouting, she spotted a crystal clear object with a little black speck darting inside in all directions. This sight interested her not because of its shiny glimmer, but because of its lively movement. As she drew nearer, she saw that it was the little fly trapped in a bottle, bouncing from side to side and buzzing frantically.

The fly spotted Raven in the distance and called up to her, "Raven, Raven, help me please!"

"Just fly up," cawwed Raven. 
But the fly was too confused in the confined space to tell the difference between up, down, sideways, frontwards or backwards and buzzed back, "I'm trapped, I'm trapped!"

Raven swooped down and tried to poke her beak into the end of the bottle to scoop the little fly out, but the hole in the top was too small for her beak to fit into. She thought of her bobbles and how she had finally found a purpose for them. However, she didn't know how to make them work.

The fly buzzed and buzzed. Raven thought and thought. She spent every day flying in circles around the bottle waiting for an answer to come to her and crying raindrops of pity for the trapped little fly.

\section{Raven Helps Fly}

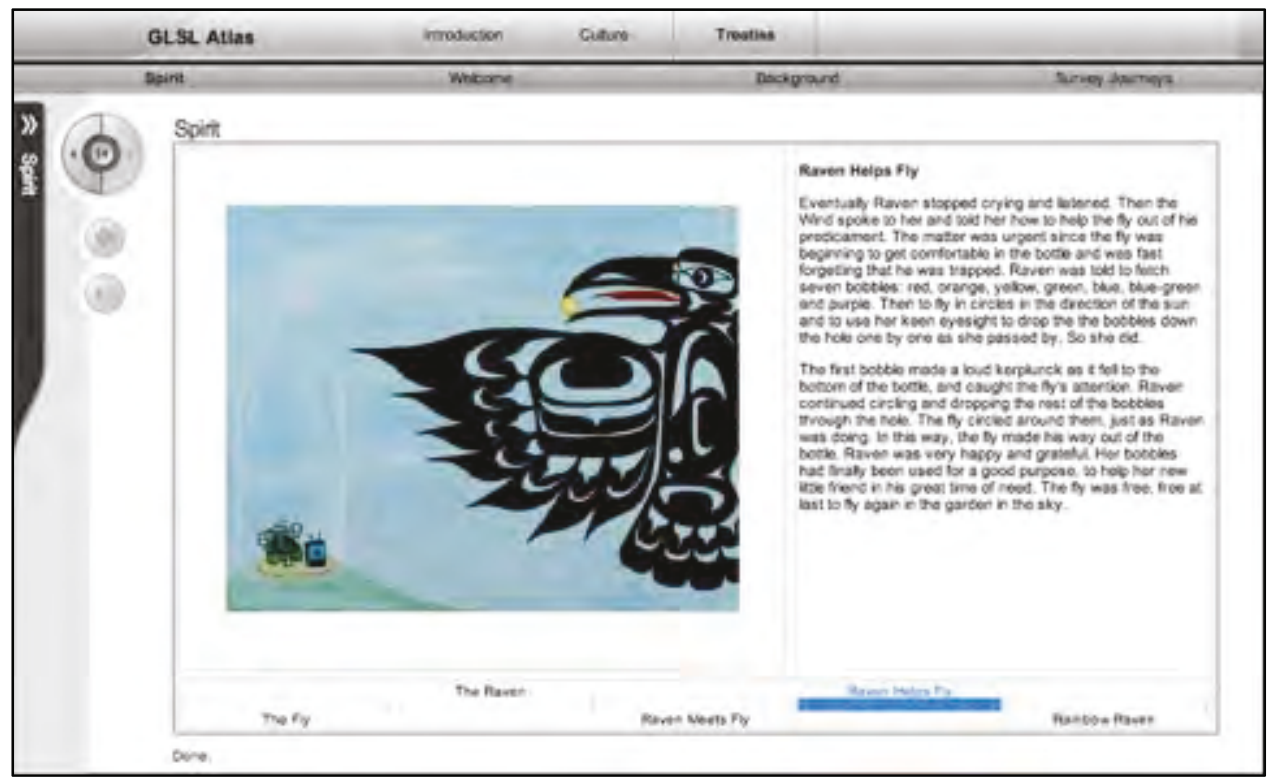

Figure F4. Screenshot showing fourth pane of Spirit Sub-module in Treaties Module of the Atlas of Indigenous Perspectives and Knowledge.

Eventually Raven stopped crying and listened. Then the Wind spoke to her and told her how to help the fly out of his predicament. The matter was urgent since the fly was beginning to get comfortable in the bottle and was fast 
forgetting that he was trapped. Raven was told to fetch seven bobbles: red, orange, yellow, green, blue, blue-green and purple. Then to fly in circles in the direction of the sun and to use her keen eyesight to drop the bobbles down the hole one by one as she passed by. So she did.

The first bobble made a loud kerplunck as it fell to the bottom of the bottle, and caught the fly's attention. Raven continued circling and dropping the rest of the bobbles through the hole. The fly circled around them, just as Raven was doing. In this way, the fly made his way out of the bottle. Raven was very happy and grateful. Her bobbles had finally been used for a good purpose, to help her new little friend in his great time of need. The fly was free, free at last to fly again in the garden in the sky.

\section{Rainbow Raven}

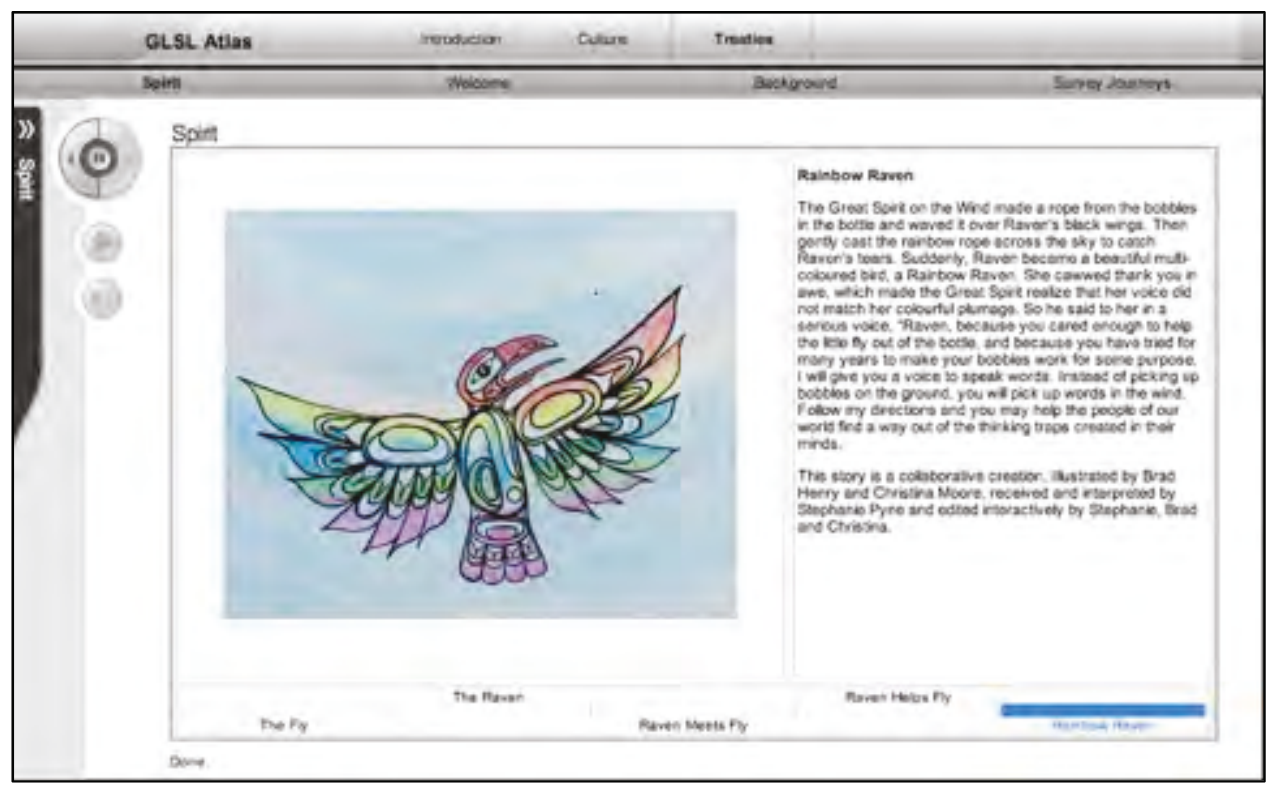

Figure F5. Screenshot showing firth pane of Spirit Sub-module in Treaties Module of the Atlas of Indigenous Perspectives and Knowledge.

The Great Spirit on the Wind made a rope from the bobbles in the bottle and waved it over Raven's black wings. Then gently cast the rainbow rope across 
the sky to catch Raven's tears. Suddenly, Raven became a beautiful multicoloured bird, a Rainbow Raven. She cawwed thank you in awe, which made the Great Spirit realize that her voice did not match her colourful plumage. So he said to her in a serious voice, "Raven, because you cared enough to help the little fly out of the bottle, and because you have tried for many years to make your bobbles work for some purpose, I will give you a voice to speak words. Instead of picking up bobbles on the ground, you will pick up words in the wind. Follow my directions and you may help the people of our world find a way out of the thinking traps created in their minds. 


\section{Preface}

"Let him who would move the world first move himself" - Socrates

Writing this thesis has sometimes been a daunting task, but in the process I have not been without support. Approaching this work from a narrative perspective is not a new endeavour. There are many people who have gone before me in this regard. In true transdisciplinary fashion, I draw on thoughts from many different people engaged in a diverse range of thinking and activities. Illustrating the relations between these thoughts and how they can be used to work together is both a central aim of this project, and I hope, a significant contribution to the work that needs to be done in this world as we find ourselves in it today. An important goal of this thesis and the atlas project it interprets has been stated time and time again by Elder Jacob Wawatie (Mowegun), when he is asked why he has devoted his life to actively sharing the knowledge he carries from his Elders: "I'm doing it for the children", Jacob says, "so that they may be able to experience the world as I have been able to".

Through the Atlas-making process my perspective has been informed by my interactions with Anishinaabek from the Lake Huron Treaty region and the broader Anishinaabek regions.

The relationship between narrative, journeying and mapping written about by David Turnbull (2007) has become clear over and over again through my Atlas-making and thesis writing journey: Story is central to understanding, story is journeying, and there is always a story behind the Story.

Through my journey in becoming a cybercartographer, I have heard many stories, told many stories, shared many stories and mapped many stories. In 
cybercartographic language these stories are referred to as geonarratives, and it is partly the task of this thesis to recount the ways in which the project to make the Atlas has both drawn on the cybercartographic concept of geonarrative, and contributed to it.

This thesis draws on a combination of previously published peer-reviewed papers, unpublished papers and chapters, and previous academic papers that I have written; it ties together and extends these works in various ways. The series of published papers, especially, mirrors the evolution of the atlas project from its inception in the fall of 2007. The purpose of this thesis is not to repeat previously completed work, but to present and extend it. Therefore, in cases where ideas from these works have been expressed with sufficient clarity, and where it is possible to smoothly weave them into the main narrative, I have included sometimes rather lengthy quotations.

During the dissertation writing process, Alan Corbiere provided me with a list of some place names in Anishinaabemowin, which I use in various places throughout the thesis. 


\section{Part I}

\section{Atlas Development in a Reconciliation Context: Introduction}

This thesis is part of a broader collaborative project to create the Lake Huron Treaty Atlas - a project that participates in the current trend in critical cartography to engage in mapping in new ways. This involves incorporating a variety of perspectives and acknowledging the situated and socially constructed nature of cartographic - and other - knowledge (Crampton, 2001; Harley, 1988, 1989, 1990, 2001; Harvey, 1973, 2000; Harvey and Chrisman, 2004; Kitchin and Dodge, 2007; Pickles, 1995a and b; Turnbull, 2007; Wood and Fels, 2008). The Atlas is becoming an effective knowledge dissemination tool through the participation of a diverse array of individuals. It is part of a new movement in public participatory GIS to mobilize knowledge by connecting, disseminating, exchanging and co-creating knowledge to meet the need for knowledge dissemination and exchange in a digital geospatial form (Goodchild, 2007a and b; Seeger, 2008).

Critical cartographers seek to rethink and redo mapping by acknowledging and transcending a colonial past (Kitchin and Dodge, 2007; Pickles, 2004; Turnbull, 2007) in which maps were used by nation-states primarily to assert and reinforce their territorial claims. Today, mapping practices are increasingly being engaged in as central components of the solutions to complex social and economic challenges (Taylor and Pyne, 2010;

\footnotetext{
6 This treaty is otherwise "officially" referred to as the Robinson Huron Treaty. However, 'Lake Huron Treaty" is used predominantly in this thesis to reflect the preference of the Lake Huron Treaty Commission to refer to the Treaty as the Lake Huron Treaty.
} 
Taylor and Caquard, 2006). The movement began with an emphasis on decolonizing history by Brian Harley (1988, 1989, 1990, 2001), who focused on deconstructing colonial maps to understand the ways they were used to exercise and promote colonial authority. Over time, critical cartographers began to look more deeply into the ontological status of maps themselves. They came to view maps as processes that give rise to emergent, "ontogenetic" knowledge and are never complete (Kitchin and Dodge, 2007). They considered epistemological issues as well, such as overcoming incommensurability in mapping Indigenous perspectives and knowledge (Turnbull, 2007).

One of the main issues in critical approaches to cartography and GIS is that geospatial technologies (Kwan, 2007) are not yet fully capable of accurately presenting multiple understandings of space, environment, and culture that are necessary in a reconciliation context (Pyne and Taylor, 2012). Concerns have been raised within the critical GIS literature with respect to the need to address the tensions that exist between GPS and GIS technologies on the one hand, and Indigenous worldviews and approaches to mapping on the other. Specifically, the positivist assumptions that are considered to underlie GPS and GIS technologies are seen by some to be incommensurable with Indigenous and postfoundationalist approaches to knowledge (Pearce, 2008; Sparke, 1998, 2005; Pyne and Taylor, 2012); such incommensurability in turn presents great challenges to the ability of geospatial technologies to reflect multiple ontologies of space (Johnson, Louis and Pramono, 2006; Pearce, 2008; Turnbull, 2007; Pyne and Taylor, 2012). There are some who reject the use of geospatial technologies in favour of mapping practices such as sketch mapping that are "personal and centered on the exploration of emotional meanings in the landscape" (Pearce, 
2008 , 1); while there are others who acknowledge the benefits of geospatial technologies but who feel that it is necessary to change the way we think about cartography (Pickles, 2004; Caquard et al., 2009; Johnson, Louis and Pramono, 2006; Pearce, 2008; Turnbull, 2007; Pyne and Taylor, 2012).

The collaborative project to create the working Lake Huron Treaty Atlas shares critical cartography's concerns with deconstruction and ontological skepticism, and aims to put some of the abstract ideas, such as "relational space" and "performativity", that are circulating in the critical cartographic (and related) literature into practice in the context of its broadest aim: Building awareness to bridge understanding by acknowledging and incorporating Anishinaabe ways in a contemporary context. This deeper aim is reflected in the Rainbow Raven and the Fly in the Bottle story presented in the Foreword, included in the Treaties Module of the Atlas of Indigenous Perspectives and Knowledge, and carried forward as part of the introduction to the Treaty Module's successor, the Lake Huron Treaty Atlas.

Phase I of the atlas project involved the production of the prototype Treaties Module under an Inukshuk Grant from May 2007-2008. The initial expansion of the Treaties Module into an atlas occurred in phase II between 2009 and 2012 under a three-year SSHRC Standard Research Grant, with the addition of 27 new working maps to complement the 3 prototype maps in the Treaties Module; and, improvements are continuing in phase III under a 2012-2014 SSHRC Outreach Grant.

The production of the Lake Huron Treaty Atlas responds to Brenna Bhandar's call to 'spatialize' history in a nonlinear, nonteleological way, [which] 
could open up possibilities for political change and transformation" $(2004,831) .7$ The project is a broad approach to reconciliation, a process that requires inclusion and involves both forward and backward looking elements, in addition to other important criteria (Dwyer, 1999). In the case of the Lake Huron Treaty process, successful reconciliation involves "revisiting" the historical geography of Lake Huron Treaty-based relationships (including their political economic dimensions) from a variety of perspectives, and identifying links between the stories of today, tomorrow and yesterday. The project to create the Lake Huron Treaty Atlas provides a way to gather these perspectives and present them in a way that questions the epistemological and ontological assumptions associated with modernism, including - but not limited to - the colonial "world view".

As a virtual geospatial public outreach and education interface, the Atlas is being designed and developed to shed light on a variety of themes over time and across space, including treaties, institutional processes, biographies and Residential Schools. In addition to the inaugural Survey Journeys Maps, which provide the basis for critically tracking the survey portion of the Robinson Huron Treaty process, the Atlas includes biography maps and other maps intended to reflect contextual details relevant to understanding the treaty story. The Residential Schools Map is a significant example of a context map that has taken on a life of its own in terms of usage and relevance to reconciliation processes that are part of the Truth and Reconciliation Commission's mandate. For example, the map is currently being used in the implementation of plans to carry out the 2013-2014 joint Aboriginal Healing Fund-Assembly of First Nations'

\footnotetext{
7 The concepts related to "spatializing history", such as "non-linear" and "non-teleological are discussed in more detail in chapters 1 and 2.
} 
National Indian Residential Schools Commemoration Project, and could serve as an innovative interface to the digital archives of the National Research Centre on Residential Schools, providing geospatially organized access to various collections.

Over the past seven years, the Atlas has been developing in an iterative manner along a variety of dimensions (Brauen et. al 2011; Pyne and Taylor, 2012; Pyne, 2013). Iterative processes give rise to emergent knowledge (Turnbull, 2007; Kitchin and Dodge, 2007; Pyne and Taylor, 2012) and represent a non-linear, cyclical view of development where past elements can be incorporated, new things can be brought forward, and certain elements can be left behind, with the possibility of re-integrating them into the development process after any number of project iterations (Cowen and Shenton, 1996; Neverdeen Pieterse, 2009; Brauen et al., 2011; Pyne, 2013). Collaborative relationships are central aspects of the types of iterative processes that give rise to the Atlas, and iterative processes occur along at least four interrelated dimensions: conceptual; financial; technological; and narrative. The iterative interplay between theory (or concepts) and practice is a hallmark of the cybercartographic atlas-making framework, which has guided the atlas project.

The central purpose of both cybercartography and the collaborative project to design and develop the cybercartographic Lake Huron Treaty Atlas is to contribute to enhanced understanding and improvement in relationships between people and between people and the land. In the case of cybercartography, it is the broader goal of achieving a new awareness of social political and economic processes through cartographic narrative; and, in the case of this specific atlas project, it is the more focused goal of enhancing awareness 
and mutual understanding in Lake Huron Treaty relationship processes. This consistency in fundamental goals has made cybercartography an ideal theoretical and methodological framework for the Lake Huron Treaty Atlas, which has involved the emergence of critical, holistic and reflexive mapping practices: seeking, presenting and reflecting knowledge in a way that incorporates Anishinaabe and critical academic perspectives.

Cybercartography is a set of concepts and tools that provides an effective atlas building framework for approaching complex social, political and economic phenomena that include reconciliation processes (Taylor, 1997, 2003, 2005, 2009, 2013; Taylor and Caquard, 2006; Taylor and Pyne, 2010; Pyne and Taylor, 2012). Cybercartography participates in the critical turn in cartography in terms of theory and application, and represents a processual (Kitchin and Dodge, 2007; Turnbull, 2007) approach to mapping and the politics of space. The cybercartographic framework makes mapping practices possible that are both responsive to and part of reconciliation processes along a variety of dimensions. The approach to maps as practices is consistent with the "ontogenetic" conception of mapping practices put forward by Robert Kitchin and Martin Dodge (2007) who "propose a radical departure in ontological thinking concerning maps: a shift from ontology (how things are) to ontogenesis (how things become), or from the nature of maps to the practices of mapping ... they are not ontologically secure representations, but, rather, a set of unfolding practices" (Kitchin, 2008, 213).

Understanding the evolution and existence of the Lake Huron Treaty Atlas with reference to "a set of unfolding practices" is not only the most apt way to describe and explain the Atlas, it is also an example of spatializing history 
(Bhandar, 2004; Brown, 2001). In addition, both the cybercartographic atlas making framework and the atlas project it supports emphasize interactivity and broad community participation (Taylor and Pyne, 2010; Pyne and Taylor, 2012). They are transdisciplinary and holistic in nature, with an emphasis on storytelling, knowledge sharing, and enhancing awareness of different perspectives. The Inuktitut name of the software developed to create atlas modules - "Nunaliit", which means "settlement", "community", or "habitat"illustrates the community orientation of the project. This name was given to the cybercartographic framework to emphasize the community based approach that was driving the development of the software in different domains: (1) open specification approaches; (2) modularity; (3) "live" data; (4) geospatial storytelling; and (5) audio-visual mapping (Caquard et al., 2009).

This thesis makes contributions in a variety of areas, including critical cartography and critical Indigenous cartographies, primarily through its critical reflections on the collaborative project to construct the Lake Huron Treaty Atlas, an online interactive multimedia or cybercartographic atlas that is continually in the making. These reflections highlight consistencies between the emergent and reflexive approach to the design and development of the Lake Huron Treaty Atlas and Anishinaabe ways that emphasize the discipline of following our hearts and of knowing and telling people who we are and where we come from.

Research, education and deliberations are occurring more frequently in web-based forums and there is an increasing amount of user-generated geographic data on the Internet (Goodchild, 2007a and b; Sieber, 2006, 2007). Volunteered Geographic Information projects are aimed at promoting broadbased education and democratic participation on issues related to culture, 
environment and sociopolitical and economic issues (Goodchild, 2007a and b; Schlossberg and Shuford, 2005; Sieber, 2006, 2007). These map-based web forums enable people to share their knowledge and perspectives on topics and issues linked to places and events, and to learn about the knowledge and perspectives of others (Craig et al., 2002; Harris and Weiner, 1998, 2002; Obermeyer, 1998a and 1998b; Sanford and Rose, 2007).

Participatory projects involving the mapping of traditional knowledge and place names are one example of the potential of these initiatives to contribute to the empowerment of Indigenous communities (Laidler et al., 2010; Tobias, 2000). The Lake Huron Treaty Atlas is another example of a geospatial, knowledge gathering and dissemination tool that is unique in the way it reaches out to a diversity of Anishinaabe and non-Anishinaabe contributors and users, giving voice to the previously marginalized and sharing knowledge across disciplines and specialty areas by mapping histories in new ways. Connecting with this diverse audience is important for enriching education, including Indigenous education; renewing relationships between youth and elders; reviving traditional culture and language; and, fostering intercultural mutual understanding.

This thesis contrasts with empirical social science research modeled on the scientific method, which tends to take a reductionist and linear approach to knowledge acquisition. Instead, I suggest shifting the focus and taking a broader, holistic view. The cybercartographic atlas project essentially comprises the field research component of this thesis. In this sense, it provides the evidence to support my thesis statement. This evidence is presented through the telling of two narratives (one in chapter 8 and the other in chapter 9), each of which derives its narrative structure and logic from the respective atlas map it narrates around, 
in addition to providing new potential content for the map. In this way, the thesis is part of the Atlas and the Atlas is part of the thesis; together, they exist in a mutually interdependent and mutually reinforcing relationship with one another.

This thesis asserts that through holistic, reflexive and critical cartographic practice, it is possible not only to acknowledge Anishinaabe perspectives in mapping processes, but to integrate them as well, in a manner that (1) preserves their inherent meaning and value, and (2) augments the meaning of the cybercartographic mapping processes. I aim to demonstrate how this type of practice has been employed in the Lake Huron Treaty Atlas by providing critically reflexive narrative accounts of the Travels in the Making of the Atlas Map and the Map of Maps, each of which functions as a narrative portal to the other maps in the Atlas.

Part I sets this project in the reconciliation context of the Seven Fires Prophecy, which is explained in chapter 1 . Chapter 2 considers the relationship between reconciliation and development in order to provide a better idea of the holistic and reflexive approach to development taken in the atlas project, and to draw attention to some of the ways this Atlas "does development"; while chapter 3 discusses in more detail the nature of the reflexive approach employed in this thesis.

Part II provides a review of some of the critical cartographic and critical Indigenous cartographic literature.

Part III examines the particular cartographic approach that frames the atlas project, cybercartography, in chapter 6 . In addition to providing a description of the atlas project that highlights its cybercartographic 
characteristics, this part provides an overview of the iterative processes that comprise the atlas project in chapter 7.

The narratives presented in part IV are inextricably intertwined with the atlas maps they interpret insofar as they draw on the narrative logic of the maps they interpret, in addition to interpreting those maps and providing new material and ideas to further populate these same maps.

Part V offers some reflections that elaborate on key bridging concepts that have been developed through this thesis research, in addition to comments regarding ongoing challenges and possibilities for the future. 


\section{Reconciliation in a Seven Fires Prophecy Context}

These are exciting and challenging times. According to the Seven Fires Prophecy of the Anishinabek (Anishinaabe people), these are times when people are beginning to seek healing and greater understanding in their relationships with each other, the land and themselves. The Seven Fires Prophecy is a Anishinaabe ${ }^{8}$ teaching that has been carried forward both through oral tradition and in the form of a wampum belt handed down across many generations. The Seven Fires Prophecy Belt was in the care of the late Elder, Dr. Grandfather William Commanda, from Kitigan Zibi, near Maniwaki, Quebec, for about 40 years until the time of his passing on August 3, 2011. During this time, Grandfather William also carried the message of intercultural peace building and reconciliation that is reflected in the Seven Fires Prophecy belt he carried.

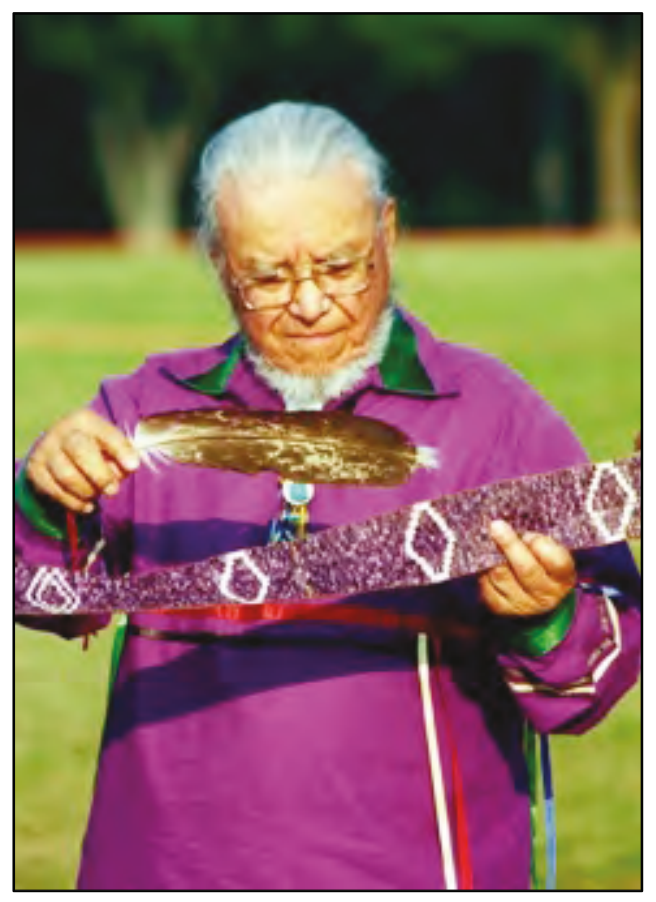

8 The first letter "a" in the word "Anishinaabe" is pronounced as a breath, so I have chosen to preface the word with the article "a" instead of "an". 
Figure 1.1. William Commanda with the Seven Fires Prophecy Belt (Prayer Vigil for the Earth web site, http: / / oneprayer4.zenfolio.com/ ?q=commanda).

The following brief description from "A Report on the Vision for the Asinabka National Indigenous Centre" (Circle of All Nations, 2010), provides an idea of the way William Commanda carried out the vision that was inspired by the Seven Fires Prophecy:

He has promoted environmental stewardship and respect for Mother Earth passionately for many decades. He conducted pipe ceremonies for the Pre-Rio Earth Summit Conference hosted by President Mitterand of France in 1991, and his prayers lie behind Agenda 21. He participated in the United Nations first Indigenous Cry of the Earth conference. He served as spiritual guide to the 1995 seven and a half month Sunbow Five Walk from the Atlantic Coast to the Pacific, to raise awareness of the growing environmental crisis; received the Bill Mason River Conservation Award in 2004; hosted workshops on water stewardship in 2004 and 2006, and 2009; is honorary chair of the Ottawa Heritage River Designation Committee; and offers interventions on current environmental issues such as the identification of the American Eel as a Species at Risk, the building of a mega dump on Danford Lake and the Navigable Waters Act (50-51).

Many have said this is the era of the seventh fire. Some even say that the eighth fire has already been lit. If this is the case, it has taken many years to gather enough kindling to get the eighth fire lit, and it could take many more to get it burning brightly. In June 2008, I told Elder Martin Assinewe from Sagamok Anishinawbek (Spanish River First Nation, Reserve No. 5) about the project to map the Lake Huron Treaty story in new ways that were critical of the colonial approach. I talked about how the project was part of the seventh fire. After listening to me, Martin responded with what I interpreted as an implicit reference to the negative, destructive - even violent - tendencies of certain worldviews when he said: "It's not only wood we have to burn in the fire" (personal communication, June 7, 2008). 
In the academic world, the kindling that will light the eighth fire of renewed relationships is a metaphor for the thoughts, ideas, concepts and approaches of people like Ludwig Wittgenstein, who stood up against the essentialism of the logical positivist movement by advocating a family resemblance approach to meaning and a therapeutic role for philosophy as conceptual clarification (Pyne, 2006). It was Wittgenstein who said:

Getting hold of the difficulty deep down is what is hard. Because it is grasped near the surface it simply remains the difficulty it was. It has to be pulled out by the roots; and that involves our beginning to think in a new way. The change is as decisive as, for example, that from the alchemical to the chemical way of thinking. The new way of thinking is what is so hard to establish.

Once the new way of thinking has been established, the old problems vanish; indeed they become hard to recapture. For they go with our way of expressing ourselves and, if we clothe ourselves in a new form of expression, the old problems are discarded along with the old garment $(1980,48)$.

In a recent conversation with Elder Lewis Debassige from $\mathrm{M}^{\prime}$ Chigeeng First Nation, Lewis pointed out how he has been seeing more and more Anishinaabe youth starting to return to traditional Anishinaabe ways, wanting to learn their language and beginning to seek knowledge from the Elders (personal communication, June 9, 2013). This trend is consistent with the message of the seventh fire prophet to be discussed below. At the same time, challenges remain, as noted by Elder Gordon Waindubence who expressed concerns about the continued influence of colonial ways in Anishinaabek Nation governance processes (personal communication, June 13, 2013). In this regard, the opening preamble of the Anishinaabe Chi-Naaknigewin (the Anishinaabe Constitution), the "Ngo Dwe Waangizid", is written in Anishinaabemowin reflecting the Dodemaag System (clan-based system of governance) and the Seven 
Grandfathers Teachings, which are also the basis for the Constitution. However, the Chi-Naaknigewin also reflects aspects of colonial governance in the form and style of (English) language used in the provisions that follow the opening words of the Chi-Naaknigewin. Gordon would like to see more chiefs learning Anishinaabemowin and incorporating "the Language" into their governancerelated proceedings. Being of the Turtle Clan, Gordon takes his time and considers many things. With this said, fundamental change takes time. Incorporating the "Ngo Dwe Waangizid" as the preamble is a good start, especially since representatives of the UCCMM Anishinabek Elder Council of Mnidoo Mnising, including Evelyn Roy, Leona Nahwegahbow and Gordon Waindubance, drafted it in Anishnaabemowin. In addition, the content of the Constitution includes specifications regarding the Dodemaag: “ ... the traditional Anishinaabe clan system of governing which is based on the Seven Sacred Gifts and informs the roles and responsibilities of the Dodemaag represented by Deer, Eagle, Crane, Turtle, Loon, Bear and Marten".10

\subsection{The Seven Fires Prophecy and the Wampum Belt}

The Seven Fires Prophecy of the Anishinaabek involves a nonlinear telling of relationships and choices across time and space. It interacts with the world and the people in it by both informing and effecting thought and action. The prophecy encoded in the wampum belt functions in significant part as a 'living' conceptual tool for interpreting and understanding history in the context of the

\footnotetext{
${ }^{9}$ See the following for more information: http://www.uccmm.ca/elders-council.html 10 See the following for more information: http:/ / www.anishinabek.ca/roj/anishinaabe-chinaaknigewin.asp
} 
present and the future. The Prophecy as written by Benton Banai (1988) tells of the messages of seven prophets at seven distinct fires, which roughly translate into epochs (Benton-Banai, 1988). This prophecy is about choices and provides guidance regarding how to interpret and understand an unfolding 'reality', including where to go and why. The Prophecy and the belt it lives through function as a map along many dimensions, including the geophysical, with geomorphological descriptions of rivers, turtle islands and flora such as manoomin (wild rice). ${ }^{11}$

The first three fires involve a prophet associated with each fire coming to the people and telling them about events that would transpire and the actions they should take (see Appendix A for the full text of the Seven Fires Prophecy, as quoted in Benton Banai, (1988)). Specifically, the people were told to continue moving west, from their initial home on the eastern edge of Turtle Island, and to continue, following the Mide (the spiritually wise people) and their interpretations of the signs indicated in the prophecies.

At the time of the fourth fire, a prophet came to the people in the form of twins, offering two sets of scenarios and choices:

The Fourth Fire was originally given to the people by two prophets. They came as one. They told of the coming of the Lightskinned Race.

One of the prophets said, "You will know the future of our people by what face the Light-skinned Race wears. If they come wearing the face of nee-kon'-nis-i-win (brotherhood), then there will come a time of wonderful change for generations to come. They will bring new knowledge and articles that can be joined with the knowledge of this country. In this way two nations will join to make a mighty nation. This new nation will be joined by two more so that the four will form the mightiest nation of all. You will know the face of brotherhood if the Light-skinned Race comes carrying no

\footnotetext{
11 The Language and Culture Map in the Lake Huron Treaty Atlas contains a series of points from
} east to west that reflect the Anishinaabek migration based on the Seven Fires Prophecies. 
weapons, if they come bearing only their knowledge and a handshake".

The other prophet said, "Beware if the Light-skinned Race comes wearing the face of ni-boo-win' (death). You must be careful because the face of brotherhood and the face of death look very much alike. If they come carrying a weapon ... beware. If they come in suffering ... they could fool you. Their hearts may be filled with greed for the riches of this land. If they are indeed your brothers, let them prove it. Do not accept them in total trust. You shall know that the face they wear is one of death if the rivers run with poison and the fish become unfit to eat. You shall know them by these many things" (Benton Banai 1988, 89-90).

The path taken at this juncture would affect the nature of the choices based on the messages of the future fires, at which the prophets foretold of the hardships the people would face over time as a result of broken relationships with the newcomers to Turtle Island (North America).

At the time of the seventh fire, which many interpret as being the current "epoch", people would begin to emerge who would be concerned with healing the broken relationships born of the past; and to do so, they would seek the wisdom of Elders in order to learn how to re-engage in relationships at all scales in healthy and balanced ways. Many of the choices spoken of at the various fires involved choices being made not only by the Anishinaabek, but by the newcomers as well, making the Seven Fires Prophecy a valuable interpretative framework for understanding how to reconcile relationships between the Anishinaabek and the newcomers to Turtle Island.

\subsection{Reconciliation in a Seven Fires Prophecy Context}

As it has turned out most of the colonial powers have loosened their grip on many of their previous "holdings" over the past century, motivated more by a desire to reduce liability rather than the motivation to promote empowerment and self-determination in "the colonies"; with the newly liberated "states" often 
left in a social, cultural, political and economic shambles. This was the case when Britain pulled out of India in 1947 with its plan to partition the country into separate states based on religious difference. As Suvir Kaul recounts in The Partitions of Memory: The Afterlife of the Division of India (2001):

Three years ago we celebrated, with a mixed sense of pride and misgiving, fifty years of national Independence. We proclaimed once again, in a number of fora, the grand narratives of anticolonial struggle and the coming of Independence, but we did not particularly engage with the political and social cataclysm that followed upon the creation of the nation-states of India and Pakistan. There is a simple and powerful reason for this: Our memories of Partition are fragmented and painful. Yet partition and its known and unknown legacies have played, and continue to play, important roles in the constitution of collective identity and thinking in India. In spite of the efforts of a number of writers and filmmakers and the work of some scholar and analysts, we remain, as a national culture, uncertain and anxious about the place of Partition in our recent history. In many ways, Partition remains the unspoken horror of our time (3).

In Canada's case, the British Crown's decision to loosen its hold by "confederating" Canada did nothing to improve Nation-to-Nation relations between "the Crown" and the Anishinaabek. For with the Constitution Act, 1867 came the division of powers between the federal and provincial "levels" of government. The opening words of section 91 assign to the Parliament of Canada "the exclusive Legislative Authority" extending to "all Matters not coming within the Classes of Subjects by this Act assigned exclusively to the Legislatures of the Provinces"; and subsection 91(24) identifies "Indians, and Lands reserved for the Indians" as one of the "Classes of Subjects" falling under this federal authority. While subsection 92(5) authorizes the provinces to enact laws concerning "the Management and Sale of the Public Lands belonging to the Province and of the Timber and Wood thereon"; and, section 109 asserts: 
All Lands, Mines, Minerals, and Royalties belonging to the several Provinces of Canada, Nova Scotia, and New Brunswick at the Union, and all Sums then due or payable for such Lands, Mines, Minerals, or Royalties, shall belong to the several Provinces of Ontario, Quebec, Nova Scotia, and New Brunswick in which the same are situate or arise, subject to any Trusts existing in respect thereof, and to any Interest other than that of the Province in the same.

The late Blaine Belleau from Gitigaan-ziibing (Garden River First Nation, Reserve No. 7) drew attention to the significance of these provisions a number of times in the many conversations I had with him since November 2007. As Blaine re-iterated a number of times, the Anishinaabek had entered into Treaty with the British and not the Canadian "Crown". They were not consulted in the several years of political jostling and maneuvering that preceded Confederation, and they certainly did not consider themselves magically to be "subjects of the Crown" upon the enactment of the Constitution Act, 1867. According to Belleau, subsection 91(24) has since been interpreted and implemented with the assumption that "Indians" are wards of the state and subjects of Canada, rather than treaty partners and members of distinctive nations. ${ }^{12}$

According to Dan Shaule (2002, 2013), also a member of Gitigaan-ziibing (Garden River First Nation), the Anishinaabek concerned with the Williams Treaty found themselves being perennially bounced back and forth between federal and provincial jurisdictions over many years in their efforts to finalize the Treaty with the Canadian government; this is because the federal government had agreed to a "basket clause", which included "other lands" that were not immediately described in the Treaty, but that were also within provincial

\footnotetext{
12 The History According to Blaine Belleau Map and the Travels in the Making of the Atlas Map in the Lake Huron Treaty Atlas contains several video clips with Blaine describing the impacts of Confederation on the Anishinaabek.
} 
jurisdiction under the Constitution Act, 1867, and therefore not negotiable by the federal government.

The legislative authority in relation to "Indians and lands reserved for Indians" that is bestowed upon the Canadian government by virtue of subsection 91(24) has "mystical foundations" (Borrows, 1999, 558, quoted in Bhandar, 2004) and is based on national "myths" concerning the Original Peoples of this land and their relationship to "Canada" (Bhandar, 2004; Borrows, 1999, 2002). These same myths have justified the Residential Schools project, a joint effort between the government and the churches, which spanned a period of at least 100 years.

On 11 June 2008, when the prime minister issued an official apology to "the former students of Indian Residential Schools," he stated the following:

The burden of this experience has been on your shoulders for far too long. The burden is properly ours as a Government, and as a country. There is no place in Canada for the attitudes that inspired the Indian Residential Schools system to ever prevail again. You have been working on recovering from this experience for a long time and in a very real sense, we are now joining you on this journey. The Government of Canada sincerely apologizes and asks the forgiveness of the Aboriginal peoples of this country for failing them so profoundly (Harper, 2011).

The statement, "there is no place in Canada for attitudes that inspired the Indian Residential Schools system to ever prevail again" (Harper, 2011), is an important statement, since it implies the desire to change attitudes. According to Brenna Bhandar (2004), such change involves letting go of the linear, teleological attitudes toward history, which she claims underlie Canadian constitutional jurisprudence, and replacing these attitudes with non-linear, non-teleological attitudes. In order to adopt a non-linear (or holistic) and non-teleological (or balance-oriented) approach to history, it is necessary to approach knowledge in a way that dispenses with many of the binary distinctions associated with the 
western worldview underlying colonialism. Important examples include the tendency in western thought to categorically separate reason and emotion, man and nature, space and place, and materiality and process (Pyne, 2006; Dower, 2000, Massey, 2004; Del Casino and Hanna, 2006).

There is increasing agreement that Indigenous peoples worldwide have much to contribute to informing the change in attitudes that needs to take place. For example, Peterson (1999) recommends adopting a both-and attitude rather than the either-or attitudes that predominate in western cultures, following the lessons he learned from central African peoples. The 730-page United Nations Environment Program (UNEP) document, “Cultural and Spiritual Values of Biodiversity" (1999), which contains Peterson's reflections, is based on knowledge that was shared at two major sets of meetings between scholars and individuals representing Indigenous communities worldwide. The document recommends not only including, but also following Indigenous ways in order to mitigate the extreme and growing crisis with respect to both species diversity and traditional Indigenous languages. The words of Klaus Topfer, UNEP's Executive Director at the time, summarize the context at the turn of the century, which remains the context of today:

As we approach the next millennium, 'globalization' has become the dominating tendency. Technology and communication systems are dissolving geographical distance and political boundaries. The positive aspects of such a trend are numerous ... However, the trade-offs are less well understood, and among these the impacts of the predominant development model on the global environment should be of major concern for us all. Climate change, loss of biological diversity, depletion of the ozone layer, pollution, exhaustion of water resources, and conflicts over shared resources are some of the most pressing problems faced by mankind.

There is strong evidence that the life support systems on which our economies depend are being overloaded, and unless a shift is made towards sustainable development we might face 
severe irreversible damage to the environment. Besides the profound ethical and aesthetic implications, it is clear that the loss of biodiversity has serious economic and social costs. The genes, species, ecosystems and human knowledge that are being lost represent a living library of options available for preventing and / or adapting to local and global change. Biodiversity is part of our daily lives and livelihoods, and constitutes the resources on which families, communities, nations and future generations depend.

\section{$[\ldots]$}

The separation of spirit from matter seems to be the prevailing philosophical approach in recent times. A re-evaluation of this precept is being shaped by the major religions of the world, in response to the global environmental crisis. This may have a profound repercussion on the way individuals and their societies perceive the environment, leading to more responsible actions.

Most indigenous and/or other traditional populations inhabit areas of mega-biodiversity. This illustrates the inextricable link between cultural and biological diversity. The very origins of environmental conservation lie buried in ancient cultures found throughout the world. Modern environmental movements express various ideologies of these original belief systems, yet do not always realize their debt to their forebears, nor towards those who still embody these ideals. Learning and respecting the ways of today's indigenous and traditional peoples, and integrating them into environmental and developmental considerations, will prove indispensable for the survival of diversity (UNEP, 1999, xi).

Norman Denzin and Yvonna Lincoln (2008) echo this view in their opening words to the Handbook of Critical and Indigenous Methodologies:

We seek a productive dialogue between indigenous and critical scholars. This involves a re-visioning of critical pedagogy, a regrounding of Paulo Friere's (2000) pedagogy of the oppressed in local, indigenous contexts. We call this merger of indigenous and critical methodologies critical indigenous pedagogy (CIP). It understands that all inquiry is both political and moral. It uses methods critically, for explicit social justice purposes. It values the transformative power of indigenous, subjugated knowledges. It values the pedagogical practices that produce these knowledges (Semali and Kincheloe, 1999, 15), and it seeks forms of praxis and inquiry that are emancipatory and empowering. It embraces the commitment by indigenous scholars to decolonize Western methodologies, to criticize and demystify the ways in which Western science and the modern academy have been a part of the colonial apparatus. This revisioning of critical pedagogy understands with Paulo Friere and Antonio Faundez $(1989,46)$ that 
"indigenous knowledge is a rich social resource for any justicerelated attempt to bring about social change" (Semali and Kincheloe, 1999, 15) (2).

The need for all people to seek the guidance of the Elders is reflected clearly in the words of the seventh prophet to the Anishinaabek:

The Seventh Prophet that came to the people long ago was said to be different from the other prophets. He was young and had a strange light in his eyes. He said, " In the time of the Seventh Fire, New People will emerge. They will retrace their steps to find what was left by the trail. Their steps will take them to the Elders who they will ask to guide them on their journey. But many of the Elders will have fallen asleep. They will awaken to this new time with nothing to offer. Some of the Elders will be silent out of fear. Some of the Elders will be silent because no one will ask anything of them. The New People will have to be careful in how they approach the Elders. The task of the New People will not be easy.

If the New People will remain strong in their Quest, the Water Drum of the Midewiwin Lodge will again sound its voice. There will be a Rebirth of the Anishinabe Nation and a rekindling of old flames. The Sacred Fire will again be lit.

It is at this time that the Light Skinned race will be given a choice between two roads. If they choose the right road, then the Seventh Fire will light the Eighth and final Fire, an eternal Fire of peace, love, brotherhood and sisterhood. If the light skinned race makes the wrong choice of roads, the destruction which they brought with them in coming to this country will come back at them and cause much suffering and death to all the Earth's people (Benton-Banai, 1988, 91).

It is becoming more and more clear that attitudes associated with colonialism have led to actions that have not only inflicted damage and hardship on the Anishinaabek, but on others as well. The words spoken at the fourth fire have come to pass: The rivers have started to "run with poison", and the fish have "become unfit to eat".

There is a need for reconciliation not only "between peoples" (intercultural reconciliation), but also between people and the land, which could be referred to as 'environmental reconciliation'. At a deeper supporting level, there seems to be a growing movement toward what might be termed 
'epistemological and ontological reconciliation'. This 'deeper' level is often concomitant with intercultural and environmental reconciliation, and achieving epistemological and ontological reconciliation is often necessary for these other forms of reconciliation to be successful (Wittgenstein, 1980). This is the space where the assumptions underlying colonialism exist, the space where critical academic thinkers spend much of their time.

The need to reconcile current and future thinking with past approaches in western thought began with a renewed and growing interest in social justice. Precipitated by the writing of thinkers like philosopher and political theorist John Rawls (1957, 1963, 1968, 1971), academics in both philosophy and the social sciences began to consider moral and ethical concerns in contrast to the strict empiricism of the logical positivism that dominated the first half of twentieth century thought. In geography, David Harvey (1973) was a leader in bringing social justice issues to the fore. Since then, critical geography has worked through the birth and development of feminist thinking, and finds expression today through such concepts as performativity (del Casino and Hanna, 2006; Thrift, 1996, 2000, 2004b; Turnbull, 2007), and relational space (Allen, 2004, Amin, 2004, Massey, 2004, Thrift, 2004a) - all in an aggregate attempt to reconcile our current and future attitudes and worldviews with those we have inherited from the past and to a great extent carry with us, unnoticed. Work in critical cartography/GIS (and in critical geography more broadly) provides an important example of attempts to achieve reconciliation in all of these areas:

Historically, scientific maps have been used by nation-states primarily to assert their territorial rights and reinforce their power over Indigenous and other communities. In contrast, various alternative maps have more recently become 'a vehicle of resistance, a language whereby rights to place may be asserted or through 
which non-dominatory representations might be cultivated" (Pickles 2004, 113, quoted in Caquard et al., 2009).

Today, after the dawn and development of thinking in critical cartography (Harley, 1988, 1989) and critical GIS, mapping practices are being pursued increasingly in the spirit of reconciliation processes with a new focus on freeing maps and mapping from the dim utilitarian light of their colonial past (Kitchin and Dodge, 2007; Pickles, 2004; Turnbull, 2007). The critical cartography literature is full of conceptual transformational thinking (for example, Kitchin and Dodge, 2007; Perkins, 2003; Crampton 2001, 2009; Turnbull, 2007; Pearce, 2008; and Wood and Fels, 2008). Being on the edge of a novel epistemological and ontological stance with respect to maps and mapping, many of the discussions in this literature are either lacking in exemplary detail or are calling on others to begin mapping from this new epistemological and ontological perspective. The Lake Huron Treaty Atlas, which includes this thesis, is a study in applying this transformational thinking to the map design and development processes. An important strand of this thinking is an approach to social science research broadly known as decolonizing Indigenous methodologies, which was coined by Linda Tuhiwai Smith (1999), and is evident in the movement toward critical Indigenous methodologies discussed in chapter 5 .

In this age of rapid biodiversity loss coinciding with equally rapid the extinction of Indigenous languages worldwide, in this age of rapid climate change and increasingly devastating "weather events", in this age of growing stratification between "rich" and "poor", in this age of impoverished water quality and declining water quantity, in this age when many changes are occurring along many dimensions, there are some in the academic community 
who have begun to incorporate Indigenous perspectives into their approaches seeking to turn things around through their research practices.

\subsection{The Seven Fires Prophecy, Reconciliation and the Lake Huron Treaty (Atlas) - Concluding Remarks}

Although, the general storyline of the Seven Fires Prophecy is quite well known, there is no substitute for the Belt carrier reading or interpreting the belt. In this case, the late William Commanda read the Seven Fires Prophecy Belt for a small group of atlas contributors, including then Chief of Magnetawan Nation, Wilmer Noganosh, Bill Allen from Burk's Falls on the Magnetawan River and me, the day after the launch of the Treaties Module in April 2008.

Elder Commanda's particular reading of the belt on that day provided an important context within which to interpret the significance of the ongoing project to tell the story of the Lake Huron Treaty process in a broad, multidimensional, cartographic context. The reading of a wampum belt is a teaching in this respect, one that is alive and responsive to the needs of the learners, and that involves subtle knowledge and insights that are distinct from (but related to) past and future teachings.

What I remember most clearly from that reading is Elder Commanda saying that people need to go back to the fourth fire and work their way back up, this time making different choices, expressing different attitudes, a direction that is consistent with the aims and approach of the Lake Huron Treaty Atlas, a project born and raised in Anishinaabe country. 


\section{Reconciliation and Development as Bimaadiziwin}

Reconciliation is "a widely accepted objective and guiding principle in attempts to deal with the aftermath of painfully repressive regimes around the world" (Bhandar, 2004, 834). It is also a process or a set of processes geared toward achieving this objective, making it an inherently reflexive concept bending back on itself and demanding a holistic approach to its implementation. Reconciliation initiatives such as commissions, investigations and hearings often include projects aimed at producing an "official truth" or "official history (Bhandar, 2004, 834). However, the 'official' nature of fact-finding commissions with their Crown appointed commissioners and their rather formal protocol for providing testimony has been critiqued as a further manifestation of colonial attitudes in the guise of a quest for reconciliation. Bhandar expresses difficulties with the extent to which a "single universe of comprehensibility" can be created through the official production of such knowledge (834); and, she asks some very relevant questions: "Who actually produces the knowledge? Who collects and interprets the data that come to form the official history? Are the data rendered through testimonials of the victims? Are they rendered through the testimony of those in power? What is left out?" (834). Bhandar questions whether or not these circumstances will lead to a merely "partial" reconciliation.

This chapter considers the relationship between reconciliation and development in order to provide a better idea of the holistic and reflexive approach to development taken in the atlas project. 


\subsection{The Call to Spatialize History in the Name of Reconciliation}

In “Anxious reconciliation(s): unsettling foundations and spatializing history" (2004), Brenna Bhandar considers the relationships between law, history, and narrative in her analysis of the mythical foundations of subsection 35(1) of the Constitution Act, 1982, which (following Derrida, 2002) she interprets as being a particularly violent constitutional provision. In the context of deconstructing the meaning, origins and basis of subsection 35(1), Bhandar provides examples of narrow Supreme Court of Canada interpretations of "reconciliation" that continue to reinforce unequal relations between the Canadian government and First Peoples, and that thereby fail to achieve reconciliation in a broad, rich and meaningful way. An important example is the Court's "long-awaited judgment in Delgamuuwk v British Columbia (3 SCR 1010, 1997)," which concerned "the right to aboriginal title under section $35(1)^{\prime \prime}$ and included a limited definition of "reconciliation":

In delineating the right, the Court stated that the purpose of section 35 (1) is to reconcile the prior presence of aboriginal peoples on the land with the assertion of Crown sovereignty (Delamuukw v British Columbia 1997, paragraph 141). The Supreme Court of Canada reiterated earlier judgments in positing 'reconciliation' as one of the main purposes behind the enactment of section 35(1). In $\mathrm{R} v$ Gladstone, the Court reaffirmed Van der Peet with respect to the objects of this reconciliation: "first, the means by which the Constitution recognizes the fact that prior to the arrival of the Europeans in North America the land was already occupied by distinctive aboriginal societies, and as, second, the means by which that prior occupation is reconciled with the assertion of Crown sovereignty over Canadian territory" (R v Gladstone 2 SCR, 723, 1996, paragraph 72) (Bhandar, 2004, 833).

Bhandar joins John Borrows in his concern that the act of defining "reconciliation" in this manner "risks undermining the very purpose of subsection 35(1) by perpetuating the historical injustice suffered by aboriginal 
people at the hands of colonizers who failed to respect the distinctive cultures of preexisting aboriginal societies" (Borrows, 1999, 548); thereby failing to "question the legitimacy of the founding violence of colonial settlement" (Bhandar 2004, 833) - which, in effect, amounts to sidestepping the heart of the matter requiring reconciliation when it comes to Nation-to-Nation relations.

Brian Egan (2012) tells the same story with different details in his discussion of the modern treaty-making process in Hul'qumi'num territory, where he regards the Crown's reconciliation process as a weak one. And, a little closer to home, in Pyne and Taylor (2012), we open with a similar commentary emphasizing the economic implications of this situation:

In today's reconciliation context, governments are publicly acknowledging their roles in past wrongs toward the Original Peoples of colonized lands and promising new approaches for the future (Dwyer 1999; Bhandar 2004). However, the continuing political and economic struggles faced by First Nations striving to create healthy treaty-based relationships with the federal and provincial governments of Canada demonstrate that achieving this objective is easier said than done. In the words of Isadore Day, Wiindawtegowinini, Lake Huron Regional Chief and Chief of Serpent River First Nation, "The truth is that one dish is empty and one is full; our treaty partner the Crown has all control and access to the wealth of our lands; and we struggle to obtain a share of the inherent wealth left to us by the Creator" ("Robinson Huron Treaty First Nations"', 2010) (92).

According to Asmal et al. (1996), a richer and more meaningful approach to reconciliation involves facing "unwelcome truths in order to harmonize incommensurable world views so that inevitable and continuing conflicts and difference stand at least within a single universe of comprehensibility" (Asmal et al. 1996, 9, quoted in Hayner, 2000, 189).

Hayner (2000) expands on what is meant by this:

In countries where simmering conflict and violence have returned in cycles, a root problem has sometimes been a fundamental 
difference in perceptions of the past. Such stark differences in understanding may keep reconciliation superficial. There is never just one truth: we each carry our own distinct memories, and they sometimes contradict each other; but debunking lies and challenging dishonest denial can go far in allowing a country to settle on one generally accurate version of history. There are some facts that are fundamental enough that broad acceptance of their truth is necessary before any reconciliation can take place (189).

The legal system, which is based on and includes the Constitution, both affects how people live and is perpetuated by their worldviews, beliefs and values. Those who speak for a full, rich and meaningful reconciliation all point to the need to include more stories and perspectives from more people, especially those who have been negatively affected by colonial processes, in order to enhance awareness and enrich the general worldview of the society in which the law exists:

In playing with and constructing different stories and strands of fiction, the objective becomes the production of "different strands intertwined in constructive friction, rather than in mere conflict and mutual strangulation" (Asmal et al., 1996, 9). The power of historical narrative in constituting the nation (and nationalism) should not be undermined. The project of reconstituting and decolonizing a colonial settler state is reliant upon the creation of new histories, in which certain strands and stories of colonial injustice and dispossession become the predominant ones (Bhandar, 2004, 834).

Pointing out the "power" of myth, Bhandar follows Borrows (1999; 2002) in recognizing the extent to which the law is founded ultimately on collective myths and worldviews:

Exploring the "mystical foundations" of sovereignty, Borrows speculates as to "what alchemy transmutes the basis of Aboriginal possession into the golden bedrock of Crown title" (1999, page 558; 2002, page 96). Alchemy seems an appropriate description for the words conjured in the form of papal bulls, royal declarations, and the proclamations of states to justify European colonialism in most, if not all, of the colonies around the world (Bhandar, 2004, 839). 
Bhandar further contends that the assertion of Crown sovereignty is based on myths that reflect a "linear", "teleological" approach to history. In contrast, she advocates "spatializing history", which means "letting go of a linear, teleological understanding of progress as moving from a point of origin to an absolute end point. The images and narratives of the past that we create or construct need to be carried into the present not as relics or artefacts to be acknowledged and observed but as living, active memory that shapes the contours of present relations between individuals and communities" (Bhandar, $2004,842-843)$.

Spatializing history involves adopting a nonlinear, holistic approach to knowledge in which the ultimate value is balance. This stands in contrast to the linear, teleological approach, which tends to be deterministic and reductionist, valuing development as progress in amassing wealth as an end in itself, and viewing history as a progression from the primitive to the more sophisticated or 'civilized'. Spatializing history means being inclusive, not only with respect to the people, voices and stories of today, but also with respect to the people, voices and stories of yesterday and tomorrow as well: "Reconceptualizing history so that the past conditions the possibility for justice in the present and future requires that the past not be contained or closed off, that images of the past are, in the words of Brown, "conceptually wrenched from temporal ordering" (Brown, 2001, page 116, quoted in Bhandar, 2004, 842).

In order to adopt a nonlinear non-teleological approach to history, it is necessary to approach knowledge in a manner that dispenses with many of the binary distinctions associated with the western worldview that underlie colonialism. In order for reconciliation to be accomplished in any meaningful 
way, colonialism itself must be transformed, not only in its approach to history, but to "development" as well.

I agree with Brenna Bhandar that a linear view of development as progress underlies the history of law in Canada, and I go further in more explicitly drawing attention to the links between the law, the land, the people and their lives. Bhandar describes how the legal basis for the Court's upholding an internally inconsistent interpretation of reconciliation is a weak, thin, even false, historical foundation: a "myth". One of the assumptions that underlies this colonial myth is the view of development as progress, and the concomitant view of development as a linear progression from the primitive to the civilized, a view with a tendency not to look back, but to surge ever onward and upward toward increasing 'sophistication' and 'proficiency'.

In contrast with the linear approach to history and development as progress, which does not look back, the "non-linear, non-teleological" approach preferred by Bhandar is mirrored in Anishinaabe understandings. For example, Algonquin Anishinaabe Elder Jacob Wawatie once shared the story his Elders told him about the Europeans with their flat-back unidirectional ships having to turn full circle in order to turn the ship around; while the Anishinaabe canoe could go in both directions with equal ease (personal communication, July 1, 2009).

\subsection{Working Toward a Holistic Approach to Development}

Michael Cowen and Robert Shenton (1996) trace the history of "development's" development. They tell the story of how it began as a holistic, lifecycle based concept in Europe, and then came to be conceived in a linear manner with the dawning of the "scientific revolution". According to Cowen and 
Shenton, this modern view of "development" included the idea of trusteeship: "Those who took themselves to be developed could act to determine the process of development for those who were deemed to be less-developed"(1996, 4). It is important to note that the view of development as trusteeship has been rejected and condemned by most (Cowen and Shenton, 1996); it is equally important to note how this approach to development was alive and well in the nineteenth century around the time of the Lake Huron Treaty signing and survey processes, which comprise the thematic entry point for the atlas project.

The development as economic growth approach emerged from the trusteeship model of development throughout the twentieth century and has been highly influential in determining economic and sociopolitical policy at all scales for many years. I referred to this approach as "bad development" in my MA thesis "Full Responsible Reason and Good Development" (Pyne, 2006), and suggested that "good" development must be based on (and involve) a holistic approach to rationality as the discipline of balancing emotional and intellectual processes, rather than the utilitarian view of rationality as the maximization of self-interest, which tends to exclude ethical considerations, and is assumed by development as economic growth approaches (Sen, 1999). In addition, these approaches often assume a scientific model of rationality that privileges prediction and control, and that further assumes neutral and objective knowledge is not only possible, but desirable as well:

By the forties and fifties "development" had come to mean economic growth and progress through exploration and innovation. The sky was the limit. Vast leaps in scientific knowledge fed technological expansion and influenced people's lifestyles. Man could fly-well and far. Life was getting easier with electric stoves, refrigerators, and television sets, not to mention such "automatic" conveniences as washing machines. At least this 
was coming to be the case in [...] societies that were lucky enough to be considered "civilized". Being "civilized" was coming to mean being modernized and being able to have all of the things that go with it. While the "civilized" societies were extracting the resources necessary to fuel this new electric lifestyle of cars, restaurants, record players, grocery stores and movies, they began to notice that the "less-civilized" societies did not seem to have as much "stuff" and were consequently less well off than they were. Development aid often arose in this context, and the term "development" itself tended to refer to resource development, economic growth and the expansion of technology (Pyne, 2006, 2).

According to the utilitarian and positivistic thinking that dominated economics at that time, development was considered primarily in terms of profits, productivity, industrial expansion and resource exploitation. Guided by this perspective, research in development economics in turn shaped the domestic and foreign policies of the "developed" nations. In The Stages of Economic Growth: A Non-Communist Manifesto (1971), W.W. Rostow describes the four stages of economic growth - the preconditions for take-off, the take-off, the drive to maturity, and the age of high mass-consumption - characterized by industrial expansion and increased production and consumption. Rostow advised "underdeveloped" countries to undergo the four stages in order to become "developed", something that would require moves toward resource exploitation and industrial expansion that were similar to what had been occurring in the more 'developed' nations:

Most of the presently underdeveloped nations, in the stage of preconditions or early take-off, must allocate much of their resources to building up and modernizing the three non-industrial sectors required as the matrix for industrial growth: social overhead capital; agriculture; and foreign-exchange-earning sectors, rooted in the improved exploitation of natural resources $(1971,139)$.

This sort of unidirectional, top-down, paternalistic and thin view of development is the same approach that has guided the Crown in its approach to 
treaty relationships with the Anishinaabek and others, making it especially important to be aware of these assumptions to ensure they are not operating in the development of the Lake Huron Treaty Atlas.

In response to the negative effects of the narrowly construed definition of "development" as economic growth (including the perpetuation of poverty, human rights violations, environmental degradation and non-sustainable business and economic practices), critics began to discuss normative theories of democracy, culture, rationality and identity in the context of development ethics (Sen, 1999; Crocker, 2003), a field of study that originated in the 1960s with Denis Goulet who stressed the importance of considering ethical as well as economic aspects of development (Crocker, 1991).

The human development paradigm, the predominant research paradigm in the field of development ethics, takes the aim of development to be "human flourishing" and focuses on how to promote and encourage this aim. The capability approach of Amartya Sen (1999) and the capabilities approach of Martha Nussbaum (2000) are both examples of conceptual frameworks within the human development paradigm. Mahbub ul Haq (1995), an important originator of the human development movement, assumes a fairly broad conception of development where "the basic purpose of development is to enlarge people's choices" (14). Ul Haq's broader approach to development as "human flourishing", "asks questions regarding development and development "beneficiaries" that are not able to be considered under traditional development as growth models:

Do they participate in economic growth as well as benefit from it? Do they have full access to the opportunities of expanded trade? Are their choices enlarged or narrowed by new technologies? Is 
economic expansion leading to job-led growth or jobless growth? Are budgets being balanced without unbalancing the lives of future generations? Are "free" markets open to all people? Are we increasing the options only of the present generation or also of future generations? (23)

The four components of human development are equity, sustainability, productivity and empowerment (ul Haq, 1995, 20). Equity is understood in terms of equity of opportunities, not necessarily in terms of results. Sustainability encompasses every kind of sustainability from resource to cultural, and is described by ul Haq as "a dynamic concept that fits our changing world [...] it is a matter of distributional equity —of sharing development opportunities between present and future generations and ensuring intra-generational and intergenerational equity in access to opportunities" (ul Haq, 1995, 18).

Whereas productivity, indexed by aggregate measures of economic growth, comprises the primary value of the development as economic growth model, it is merely one of the components of development as the expansion of people's choices and opportunities: "An essential part of the human development paradigm is productivity, which requires investments in people and an enabling macroeconomic environment for them to achieve their maximum potential" (ul Haq, 1995, 20). The fourth, though not least important, component of human development is empowerment. The human development paradigm is non-paternalistic; that is, it does not assume a superior normative position and it focuses on development by the people who must participate in the activities, events and processes that shape their lives" (ul Haq, 1995, 20). Ul Haq refers to empowerment as a comprehensive concept: "Empowerment means that people are in a position to exercise choices of their own free will. It implies a political democracy in which people can influence decisions about their lives. It 
requires economic liberalism so that people are free from excessive economic controls and regulations" $(1995,20)$.

Amartya Sen's capability approach incorporates some or all of the above components in its conception of human development. In fact, in Development as Freedom (1999), Sen goes so far as to define development in terms of freedom (empowerment): "Development is the freedom of people to live the lives they have reason to value" (85). David Crocker (2003), describes Sen as "the most important practitioner of development ethics emerging from economics in general and development economics in particular" (465). In addition to the valuable contributions Sen has made by providing a richer and more accurate mode of thinking about human development than the utilitarian, development as economic growth approach in particular, Sen's approach has inspired an exciting multidisciplinary dialogue and represents a step forward in attempts to make sense of and improve upon the human condition (see for example Gasper, 2002; Giri, 2000; Crocker, 2003; Alkire, 2002). In general, Sen's work has contributed to a "more enriched, more ethically aware economics...a philosophy that is more rigorous and policy-oriented, [and] less narrowly Euro-American ... in its assumptions and concerns" (Gasper, 2000, 989).

Consistent with the aims of the human development paradigm, Sen characterizes his freedom-oriented capability approach as a broad approach that integrates economic, social and political considerations (1999, 8). In Inequality Reexamined (1992), Sen defines well-being in terms "of the quality...of a person's being" (p. 39). This "quality" is further defined in terms of functionings. A person who "achieves" a certain level of well-being is considered to have "a set of interrelated functionings, consisting of beings and doings" (39). Sen provides 
examples of functionings that range from " being adequately nourished...to more complex achievements such as being happy, having self-respect, [and] taking part in the life of a community" (39). The concept of capability is related to "functioning" since "it represents the various [potential] combinations of functionings (beings and doings) that the person can achieve" (40). Emanating from these basic conceptual distinctions, Sen develops a "language" of interrelated concepts intended to work together both in theory and application in the pursuit of development as freedom.

While the capability approach has opened up the conceptual terrain of development on one hand by allowing for the discussion of human values, it has been criticized for being either too vague or too ambiguous in certain respects. In other words, the view is emerging on many fronts that Sen has not gone far enough in his approach toward assuring the ultimate end of human development: human flourishing. Sabina Alkire maintains that Sen's approach is "deliberately incomplete", in order to provide an approach with broad scope and diverse applicability (2002). Sen realizes that his approach is general, but hopes that it can open up the conceptual terrain of development for further more specific theories; and, he urges people not to wait until models of justice are completely "fine-tuned" before we make determinations concerning the justice or injustice of particular cases: "Rather, what is needed is a working agreement on some basic matters of identifiably intense injustice or unfairness" $(1999,254)$.

There have been many efforts to extend or supplement the capability approach (Gasper, 2002; Alkire, 2002; Nussbaum, 2000; Crocker, 1991, 2003, 2008; van Staveren, 2001), and various limitations have been identified (Pressman and Summerfield, 2002; Giri, 2000; Carmen, 2000; van Staveren, 2001). Some feel that 
Sen's approach retains traces of moral individualism; others claim that Sen fails to adequately explore the nature of self. There are questions about Sen's focus on the value of freedom and about his characterization of freedom, in addition to concerns about Sen's conception of rationality. Despite the apparent diversity, these concerns are related.

While there is much agreement that Sen's capability approach goes further than mainstream approaches in economics, there is some concern that Sen has not gone far enough in his examination of the 'human' in human development. Des Gasper is concerned with what he considers to be the thinness of the concepts of well-being and agency in the capability approach, and recommends Irene van Staveren's (2001) moral capabilities, which are essential for a person "to be able to interact with others, and to form purposes and then care about, commit to and act on these, and to deliberate about the tensions and balancing between different incommensurable values" (Gasper, 2002, 451).

Charles Gore (1997) credits Sen with going beyond the traditional welfarist view of the person as a "spoon-fed patient" by introducing "freedom of choice"... into well-being assessment", but he maintains that Sen has not gone far enough since "the processes of forming objectives, participating in shaping the conditions for action, and making things happen, are all divorced from what well-being is" (Gore, 1997, 241)". By moving from utility to functionings in his evaluative approach, Gore believes that Sen "has gone in the right direction but stopped in the wrong place". According to Gore, "judging well-being in terms of disembedded functionings is equally `a highly limited way of seeing people'. It is necessary to move a step further and recognize the embeddedness of intentional 
and reflexive activity within given, but changing, institutional contexts as being intrinsically important for well-being"' (Gore, 1997, 246).

Gore's (1997) communitarian critique of the capability approach suggests that the capability approach is morally individualistic in two ways since "the goodness or badness of social arrangements or states of affairs is evaluated on the basis of what is good or bad for individual well-being and freedom and is also reduced to the good of those individuals " $(1997,241)$. According to Gore, there is a class of "social goods" that is shared by all in a group and not divisible among individuals. These "irreducibly social goods" are intrinsically valuable and are essential in the determination of human well-being, and range from moral norms and "modes of discourse" to modes of government and contribute to the meaning of experience. Gore's inclusion of irreducibly social goods provides an explanation for why two similar activities can have different meanings. For example, consider the contrast in meaning between simply watching or looking at birds and the "institutionalized" pastime of "bird-watching as an activity that has a specific character in society where there are particular attitudes to nature and animal tracking" (Gore, 1997, 244).

Ananta Giri (2000) enriches Sen's concept of "well-being" by considering the relationship between happiness, wellness of being and self-development, emphasizing the contribution of state-of-being happiness to well-being: "Thus to strive for happiness is not to run after momentary pleasure alone but to strive to realize equanimity in life, which enables one not to lose one's peace of mind when confronted with either adversity or affluence" (Giri, 2000, 1007). State-ofbeing happiness is especially significant for well-being, since the "quest for happiness as realization of peace of mind provides a more secured and 
sustainable subjective foundation to the objective quest for human well-being" (Giri, 2000, 1007). Giri "salutes" Sen for being concerned with the welfare of the disadvantaged, but maintains, "the...quest [for well-being that includes happiness as peace of mind] is not unimportant for the poor and deprived as well" (2000, 1007). Giri sees self-cultivation, which involves the ability to attain state-of-being or peace-of-mind-happiness, as an important dimension of human development since it "provides a bridge between the well-being aspect and the agency aspect of the human person" $(2000,1009)$.

Raff Carmen (2000) presents the most radical critique of the capability approach, extending his critique to all "abstract formal" approaches to development, which he claims are "outside-in" approaches that cannot help but promote dependency and inhibit true human flourishing. Carmen paints a dismal picture of development from the outside in:

The excluded [are not] well-served with development intervention packages which are 'not theirs', nor with projectile projects targeted 'at' them from the outside. Those interventions and those capital-intensive outside expert-led and outside-funded projects, be they of GO or NGO ilk have been shown for five long disheartening development decades, to have served - and very efficiently so - the tastes and 'capabilities' for self-preservation, self-aggrandizement, self-promotion and institution-building ('empire-building') of the interventionists and of the self-appointed assorted modernizers, extensionists, civilizers and handsomely remunerated trainers, capacity builders, empowerers and developers, and their respective organizations themselves, often without increasing by one iota sometimes quite the reverse - the capacity for sustained and sustainable autonomous human agency of those being intervened the 'target' populations and so-called 'beneficiaries' (2000, 1021-22).

Carmen (2000) describes himself as someone who "spent half a lifetime looking from the outside in, i.e. from the engine rooms of knowledge and power (in academia and in a large multinational)", until he decided to "step in the mud" (2000, 1020). Discussing the effect of a simple change in perspective - looking 
from the 'inside out', instead of from the 'outside in' - Carmen echoes the view that development discourse in terms of functionings and capability is "light years removed from the soulless and culturally disembedded shibbolets of economic development transfer", (2000, 1021); and, he acknowledges the reformative impact this discourse has had on "growth as development policies". $(2000,102)$ However, he cautions these concepts are about "what ought to be" and "what ought to be done", rather than about how. According to Carmen, the capability concept is limited because it is presented in "static, non-process language" and it is "a concept relatively closer to an individualistic mindset". The danger of this mindset is that it promotes an us-them attitude with respect to the relationship between "donor" agents and donation recipients and discourages the development of "autonomous human agency", at the same time imposing a "foreign intellectual framework" in the name of furthering local autonomous agency. In contrast, Carmen presents a "growth and learning" conception of capability stating "capability can be enhanced by (i) human learning and (ii) cooperation and solidarity with others" $(2000,1023)$ in an equitable environment of peers. Carmen insists this dynamic, process oriented, "growth and learning" model of capability can itself be a powerful approach to development thinking. Consistent with his distinction between an 'outside in' and an 'inside out' perspective, Carmen contrasts the "culture of power" with "power of culture", where the "culture of power" is an "isolationist attitude" that does not "need the other" and the "power of culture" is powerful "because of the factor of solidarity (cooperation with others...) and because it has the dynamic, creative capacity to learn" $(2000,1023)$. 
Carmen's "capacitation" represents an improvement over 'capability' since "capacitation ... does not have to wait until power, income and employment (and the freedom to choose which naturally goes with them) [are] delivered, provided and transmitted ... Capacitation has the power - by virtue of learning in solidarity with others - to autonomously generate employment and income" $(2000,1023)$. Carmen's basic criticism is that the static nature of such concepts as agency freedom and agency capability perpetuate the distinction between donors and the excluded as beneficiaries whose agency depends on the benevolence of those more "fortunate". For Carmen, true human autonomous agency can only be achieved by learning in cooperation with others:

Agency then means "the ability to act, this being a precondition of creative knowledge" (Paulo Freire). Education itself, not least when understood as 'learning in solidarity with others' can therefore be conceived of as a form of 'agency', i.e. an act of co-operative knowledge creation (in contrast with the linear, individualized transmission of knowledge and skills which underpins the concept and practice of 'training'). 'Agency', 'agency capability' and 'agency freedom' therefore not only 'belong' to the 'providing', 'intervening', 'assisting', 'delivering', 'transmitting' and poverty alleviating External Mover (Institutions), but (also) to autonomous human agents by virtue of the simple fact of their being human(s) and provided they act in solidarity $(2000,1025)$.

Similar to Carmen's inside out approach is the 'development from within" approach (Taylor and McKenzie, 1992), which emphasizes the role of participation and is contrasted with 'development from below' approaches. In addition to other critiques, "considerable skepticism was expressed by some Indigenous planners who said that development from below and concomitant ideas, such as agropolitan development, were just one more example of theories and prescriptions which are developed in the North applied to the South" (Taylor, 1992, 234). 


\subsection{Applying a Holistic Approach to Development as Bimaadiziwin in the Making of the Lake Huron Treaty Atlas - Concluding Remarks}

The concept of "development" is central to the atlas project, which takes an emergent and holistic approach to the collaborative geonarrative creation. This iterative approach to development incorporates past elements, brings new things forward and leaves certain elements behind, with the possibility of reintegrating them in the future (Cowen and Shenton, 1996; Nederveen Pieterse, 2009). From an inside out, development from within perspective, this atlas project has involved 'spatializing history' by (i) "re-visiting" the historical geography of Lake Huron Treaty-based relationships from a variety of perspectives to reflect their various dimensions; (ii) questioning the epistemological and ontological assumptions associated with the colonial worldview; (iii) employing a collaborative investigative approach involving broad community participation; and, (iv) using Anishinaabe teachings (kinoomagi) involving the past, the present and the future, as guides in the atlas design and development process.

I have let the Anishinaabe way be my guide in much of the atlas-making process in my role as geonarrative and content editor (see chapter 7). Bimaadiziwin, which translates roughly into "the Good Life", has been described by Lawrence Gross as "a long and healthy life" (2002, 15-16). Gross provides a brief and general description of Bimaadiziwin:

The teaching of Bimaadiziwin operates at many levels. On a simple day-today basis, it suggests such actions as rising with the sun and retiring with the same. Further, Bimaadiziwin governs human relations as well, stressing the type of conduct appropriate between individuals, and the manner in which social life is to be conducted. Bimaadiziwin also covers the relationship with the broader environment. So, for example, it teaches the necessity of 
respecting all life, from the smallest insects on up. Bimaadiziwin, however, does not exist as a definitive body of law. Instead, it is left up to the individual to develop an understanding of Bimaadiziwin through careful attention to the teaching wherever it can be found. This makes the term quite complex, and it can serve as a religious blessing, moral teaching, value system, and life goal. The reader should be forewarned, then, that the term appears in all these guises below. Most importantly, this protean character allows Bimaadiziwin to flow through just about every facet of traditional Anishinaabe [life].

If we follow the course of an Anishinaabe from birth to death, Bimaadiziwin is present throughout. In childhood, the most important exposure to the teaching comes through the telling of stories. The myths of the Anishinaabe are one of the great depositories of moral teaching. On the surface, this may not appear so. The stories of the culture hero, Wenabozho [aka Nenboozhoo] are often humorous and entertaining. Also, Anishinaabe storytellers never conclude with a moral as in Aesop's Fables. The narrator allows the listener to develop an individual understanding of the lesson being conveyed. As such, the stories are designed to engage the listener, implanting seeds for later reflection and contemplation $(2002,19)$.

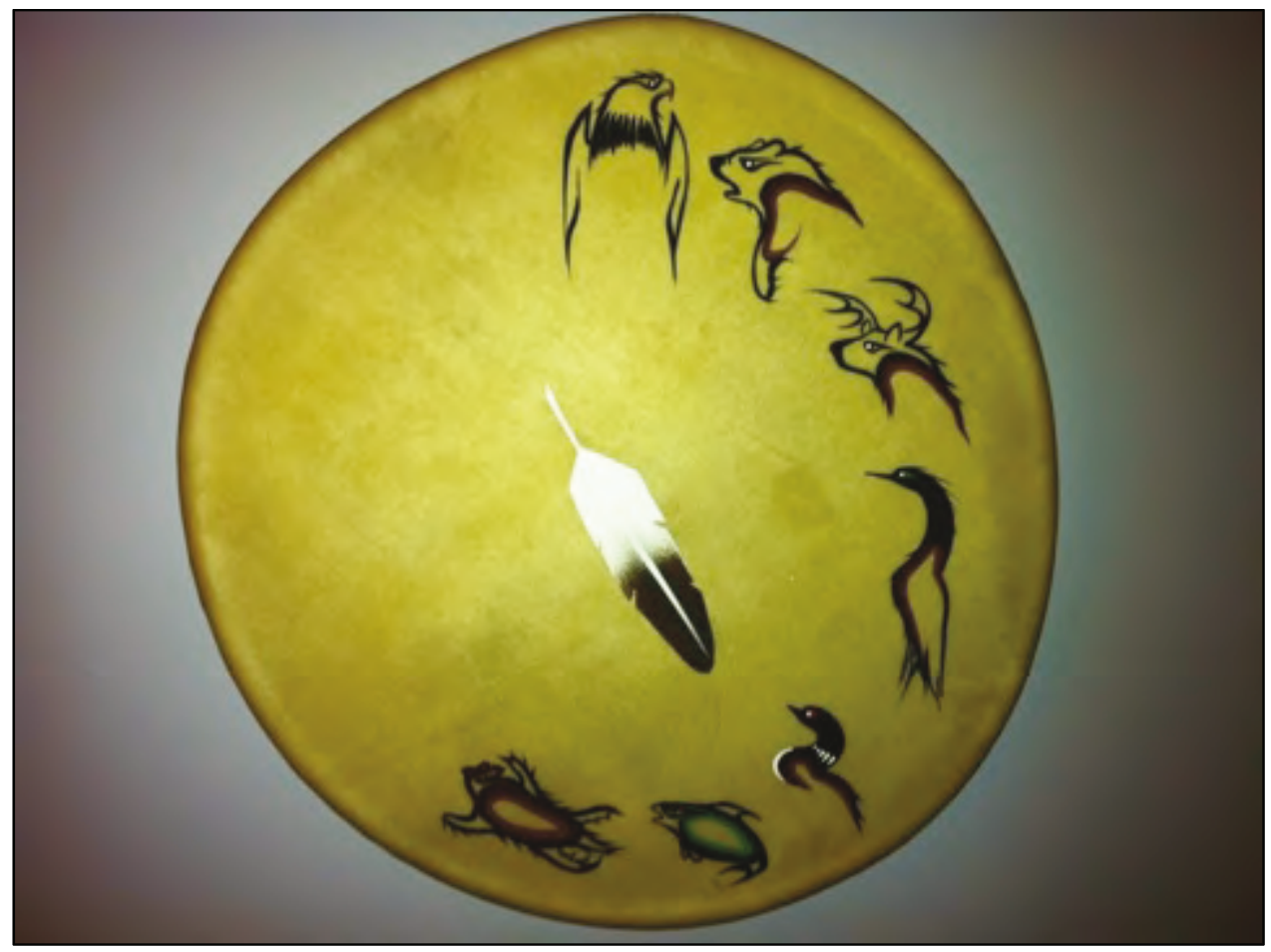

Figure 2.1. The Atlas Drum - Hand drum made for me by Isabelle Meawasige and Blain Commanda (Serpent River First Nation) and hand painted by Stan Panamick (M'Chigeeng First Nation). 
The Anishinaabek were given the Seven Grandfathers Teachings of wisdom, love, respect, bravery, honesty humility and truth to help them live long and healthy lives, in addition to the 13 Grandmother Moons teachings. In October 2012, Guy Dumas gave a presentation on the Seven Grandfather's teachings, in which he drew some important links between these teachings and the insights of several scientists. Seeing the similarities between Dumas' conceptual method and the two-pronged approach to the Atlas-making process, I video-recorded the talk, which I plan to upload to the Atlas (see Appendix D for digital audio recording).

Throughout the Atlas-making process, I have been gathering insights from various individuals and incorporating them into the atlas in various ways. Examples include Grandmother Josephine Mandamin, who has led many Water Walks around the Great Lakes and beyond, and who talked about the Anishinaabe way of following your heart through action when inspiration hits. ${ }^{13}$ There is also Gimaah Wilmer Noganosh whose life story reflects the theme of working together; Eddie Benton Banai who teaches clan-based governance to his students through a combination of practical experience and investigations into the past; Elder Joseph Laford with his often spoken reminder that we are Spiritual Beings having a Human experience and not human beings who sometimes have spiritual experiences; the late Elder Blaine Belleau who relayed the wisdom that his father had passed on to him: "If you tell the truth, you can never get caught in a lie". There is also Lewis Debassige whose living knowledge of Anishinaabe language and culture combined with rich institutional knowledge

13 For more information on the Mother Earth Water Initiative see http://www.motherearthwaterwalk.com 
continues to serve his community well, especially in the areas of language, culture, governance and education. Linked to this is the supportive role of the Ojibwe Cultural Foundation (OCF) under the former directorship of Alan Corbiere, and includes Alan's ongoing interest and beneficial influence in the project (discussed further throughout this thesis). ${ }^{14}$ Gordon Waindubence is a Mnidoo Mnising Grand Council Elder whose teachings have provided an ongoing source of insight and inspiration in the Atlas-making process. Among many other individuals who have contributed to the Atlas so far, it is imperative to mention Nenboozhoo, the Anishinaabe "trickster", who takes an active interest in the affairs of the world that extends even to the farthest reaches of its legislative dimension (Debassige and Pyne, 2012; Borrows, 2002). These brief descriptions provide a glimpse of the types of insights into Bimaadiziwin that I have gathered along the way and tried to incorporate into my approach to developing the Atlas as a reconciliation tool.

The Lake Huron Treaty Atlas is being developed in response to the need for authentic or meaningful reconciliation in Lake Huron Treaty-based relationships. In contrast with the processes involved in producing some "official histories", a holistic approach to the development of geonarratives involving diverse participation contributes to the type of understanding and awareness that is required for successful reconciliation to occur. The Seventh Fire teaching recommends following the Anishinaabe way in order to reconcile relationships in a meaningful way that is consistent with Bimaadiziwin. My awareness of this teaching has influenced me in the way I have implemented the two-pronged approach to Atlas development.

14 For more information about the OCF see http: / / www.ojibweculture.ca/ 


\section{Crystallization in the Eighth Moment: Thesis Method ${ }^{15}$}

This thesis falls within the vast space of qualitative inquiry, which includes "interpretive and critical paradigms" and "complex epistemological and ethical criticisms of traditional social science research" (Denzin, 2001, 324). The Introduction to the Handbook of Qualitative Research provides a useful description of qualitative methods that identifies eight historical moments:

Qualitative research is a field of inquiry in its own right. It crosscuts disciplines, fields, and subject matters. A complex, interconnected family of terms, concepts, and assumptions surround the term qualitative research. These include the traditions associated with foundationalism, positivism, postfoundationalism, postpositivism, poststructuralism, and the many qualitative research perspectives, and/or methods connected to cultural or interpretive studies ... There are separate and detailed literatures on the many methods and approaches that fall under the category of qualitative research, such as case study, politics and ethics, participatory inquiry, visual methods, and interpretative analysis.

In North America, qualitative research operates in a complex historical field that crosscuts at least eight historical moments ... We define them as the traditional (1900-1950); the modernist, or golden age (1950-1970); blurred genres (1970-1986); the crisis of representation (1986-1990); the postmodern, a period of experimental and new ethnographies (1990-1995); postexperimental inquiry (1995-2000); the methodologically contested present (20002004); and the fractured future, which is now (2005-). The future, the eighth moment, confronts the methodological backlash associated with the evidence-based social movement. It is concerned with moral discourse, with the development of sacred textualities. The eighth moment asks that social sciences and the humanities become sites for critical conversations about democracy, race, gender, class, nation-states, globalization, freedom, and community (Denzin and Lincoln, 2005, 2-3).

Although the temporal periods referred to in Denzin's account of the eight

historical moments of qualitative research differ from the far older Seven Fires

\footnotetext{
${ }^{15}$ Special acknowledgement to Trent Falldien (J.D.) from Atikameksheng Anishnawbeg traditional territory (Sudbury) for suggesting the relevance of symbolic interactionism to my thesis project, a suggestion that helped me greatly in identifying a reflexive approach as the methodological lens for this dissertation, and that has also given rise to insights into future research.
} 
Prophecy, which reaches back at least 600 years, it is important to remember that the Seven Fires Prophecy is not necessarily linked solely to a linear approach to time. From a Seven Fires Prophecy perspective, I interpret Denzin's eight historical moments as an evolution in thought with the potential of contributing to the 'lighting of the eighth fire' discussed in chapter one. The two related turns in thinking that have occurred in this historical evolution are the "spatial turn", with an emphasis on performance, process and relationships (to be discussed in chapter 4); and, the "interpretative turn", with its emphasis on narrative and reflexivity (Denzin and Lincoln, 2005): "In both inductive analytic ... and more artistic approaches to qualitative research, researchers [have] abandoned claims of objectivity in favor of focusing on the situated researcher and the social construction of meaning" (Ellingson, 2009, 1-2). Subjective bias, as it is understood in a conventional science sense, does not exist according to the view of "research" that is emerging in this eighth moment.

The interpretive view is moving toward a holistic perspective from which the binary distinction between subjectivity and objectivity does not exist. This is not to say that the individual's perspective does not matter, however, quite the contrary. Instead we have the "situated researcher", a concept more akin to the Anishinaabe view: An individual with a unique perspective and gifts [...], with the capacity for vision, and a responsibility to develop that vision and those gifts for themselves - including their Spirit - and their community.

The Lake Huron Treaty Atlas has emerged in a way that is consistent with decolonizing Indigenous methods and other complementary critical academic perspectives. The thesis portion of the Atlas goes further than the broader atlas project to the extent that it incorporates a reflexive narrative approach to 
interpret various aspects of the project. This chapter points out affinities between the approach taken to this thesis, and the overall atlas project, and decolonizing Indigenous methods, emergent methods, reflexive narrative method and qualitative inquiry as crystallization. The reflexive narrative method employed in this thesis is both reflective and reflexive. Reflection is a necessary phase of the iterative processes that have gone into the making of the Lake Huron Treaty Atlas, which will be discussed in chapter seven and subsequent chapters in this thesis; while reflexivity involves reflection, and can be applied in many areas, also to be discussed at further length throughout this thesis. For now it is sufficient to say that reflexivity in social science research mostly commonly has to do with the importance of a person's identity and position with respect to the research relationships they engage in, but also refers to a certain kind of relationship between entities that have similar form but that differ in scale or dimension. Benoit Mandelbrot's (1983) "fractals" exhibit these properties, water, mountains, trees and the human body being significant examples.

\subsection{Qualitative Inquiry and Crystallization}

In his unwavering focus on the aim of social transformation as a goal for qualitative inquiry, Norman Denzin rejects over-specified approaches to method and recommends approaches that are sensitive to local particularities and context:

Can we have a moratorium on mixed methods talk about designs and typologies and get back to the task at hand, which is changing the world? The bricoleur is in the business of changing the world for social justice purposes. We must act as catalysts for social change. History, change, and transformation belong to those who care, who remember, who struggle to re-remember, who turn history back against itself, who expose the cracks and contradictions in history itself (Smith, 2004). The goal is to provoke change, to create texts that play across gender and race, utopian 
texts that involve readers and audiences in this passion, moving them to action $(2012,85)$.

The "bricoleur" is not bound by any given method or combination of methods prior to embarking on their research "quest". In line with this thinking, Denzin recommends a crystallized vision of method:

Multigenre crystallization is Ellingson's (2009) postmoderninfluenced approach to triangulation. She disputes a narrow conception of triangulation. She endorses a postmodern form, asserting that the central image for qualitative inquiry is the crystal, multiple lenses not the triangle. She sees crystallization as embodying an energizing, unruly discourse, drawing raw energy from artful science and scientific artwork. Mixed-genre texts in the postexperimental moment have more than three sides. Like crystals, Eisenstein's montage, the jazz solo, or the pieces in a quilt, the mixed-genre text combines "symmetry and substance with an infinite variety of shapes, substances, transmutations ...crystals grow, change, alter ... crystals are prisms that reflect externalities and refract within themselves, creating different colors, patterns, arrays, casting off in different directions (Richardson, 2000, 934)"(Denzin 2012, 83-84).

According to Laura Ellingson, "crystallization combines multiple forms of analysis and multiple genres of representation into a coherent text or series of related texts, building a rich and openly partial account of a phenomenon that problematizes its own construction, highlights researchers' vulnerabilities and reveals the indeterminacy of knowledge claims even as it makes them" $(2009,4)$. This approach to method best approximates the approach to the design and development of the Atlas, including this thesis, especially insofar as it does "not promote a rigid, recipe-like, or formulaic approach to crystallization, but instead [seeks] to provide a map of the terrain to guide those seeking to learn more, who could benefit from the specificity and instructions" (Ellingson, 2009, 4).

The concern of a crystallized approach for highlighting vulnerability and knowing when and what to report and how to report it is similar to the concern 
many Anishinaabe Elders have for not revealing too much without knowing who it is being revealed to, and where and how it is being revealed. This in turn highlights the importance of face-to-face contact for some knowledge sharing and of knowing who you are talking to and the situation in which the conversation is taking place. There is an aspect of judgment here that could benefit from the wisdom of the Elders.

\subsection{Reflexive Methods}

Early in Linda Finlay and Brendan Gough's (2003) discussion of reflexivity, they point out how "the etymological root of the word 'reflexive' means 'to bend back upon oneself'" (ix), and identify some of the main strands of investigation involving reflexivity, including "the humanistic-phenomenological and psychoanalytic emphasis on self-knowledge, 'critical' traditions such as feminism, which prioritize socio-political positions, and social constructionist and 'postmodern' approaches, which attend to discourse and rhetoric in the production of research texts "(1).

According to Ann Cunliffe (2003), the critical turn to questioning the possibility - and even desirability - of a neutral, objective, "scientific gaze" has led social science practitioners to "a reflexive stance in which we recognize all social activity, including research itself, as an ongoing endogenous accomplishment. Three main themes have emerged: a crisis of representation, an emphasis on the constitutive nature of language, and a call for reflexive approaches to research" (983). Cunliffe takes a broad view of reflexivity, which suits this project well:

The notion of reflexivity, or a crisis of truth, has emerged within many disciplines: philosophy (Derrida, 1976; Heidegger, 1966), linguistics (de Saussure, 1959; Wittgenstein, 1953), the natural 
sciences (Ashmore, 1989; Latour, 1988), anthropology (Clifford and Marcus, 1986), sociology (Bodily, 1994; Garfinkel, 1967; Gouldner, 1970; Pollner, 1991), psychology (Gergen and Gergen, 1991; Shotter, 1992), and comparatively recently in organization and management studies (Alvesson and Sköldberg, 2000; Calás and Smircich, 1999; Cooper, 1990; Watson, 1995). Essentially, reflexive scholars question the threads of philosophical and methodological certainty implicit in the goal of mainstream social science to provide an absolute view of the world. They suggest that all forms of inquiry are paradigmatically circumscribed (Burrell \& Morgan, 1979; Chia, 1996), and therefore truth claims, assumptions about reality and the ways in which we generate accurate theories should be challenged to reveal the inherent instability of knowledge (983).

In "Research and Indigenous participation: critical reflexive methods" (2009), Ruth Nicholls recommends participatory and collaborative methodologies for research involving Indigenous peoples. According to Nicholls, "researchers need to engage with reflexive evaluation of collective and negotiated design, data collection and data analysis to consider inter-personal and collective dynamics during the research process" (117). Nicholls advocates applying multiple layers of reflexivity in research involving Indigenous people:

Additional political and relational layers of reflexivity are essential to critically evaluate empowerment and participation by working 'the spaces between' through reflection about collaboration. By exploring 'relationality' as a methodology, this paper discusses three layers of reflexivity (self-reflexivity, inter-personal reflexivity and collective reflexivity). This reflexive work entails resisting essentialist positions while also recognising difference within a collective. The result is the ability to see that the Self-Other hyphen both connects and distinguishes between us, in processes of collaborative counter-colonial research (117).

Due to the multidisciplinary roots of "reflexivity", "questions arise over whether it is a philosophy, a research method or a technique, and numerous types of reflexivities have been identified (e.g. Holland, 1999; Latour, 1988; Lynch, 2000)" (Cunliffe, 2003, 984); therefore Cunliffe begins with Pollner's "radical reflexivity" as "an 'unsettling,' i.e., an insecurity regarding the basic 
assumptions, discourse and practices used in describing reality" (Pollner, 1991, 370) (Cunliffe, 2003, 985):

Reflexivity 'unsettles' representation by suggesting that we are constantly constructing meaning and social realities as we interact with others and talk about our experience. We therefore cannot separate ontology and epistemology, nor can we ignore the situated nature of that experience and the cultural, historical, and linguistic traditions that permeate our work (Cunliffe, 2003, 985).

Cunliffe provides a definition of "reflexivity" that situates "reflexivity"

between the "two root metaphors [of] otherness and betweeness":

Texts grounded in otherness build on postmodern and poststructuralist commitments by incorporating deconstructionist or contradiction-centered approaches (Hatch, 1997; Steier, 1991). This metareflexive approach addresses the representational crisis head on, by using the oppositional logic of otherness to overturn any notions of our ability to produce truthful, authoritative accounts. Texts grounded in betweeness draw on ethnographic work, specifically constructionist approaches, proposing that social realities are constructed between us in our conversations (Shotter, 1993; Watson, 1994). Each metaphor has very different implications for researching organizational life (Cunliffe, 2003, 986).

Both perspectives "define reflexivity as a turning back" (989), but differ in

terms of their focus:

Whereas deconstructionist approaches carry an epistemological stance concerned with problematizing explanations and revealing philosophical, ideological, linguistic and textual uncertainties, constructionist approaches focus on our ways of being and acting in the world, how we make sense of our experience, and therefore call for narrative circularity - tracing the situated and partial nature of our accounts. The former throws open debate on issues such as the philosophical suppositions underlying texts; the latter on ontological issues of who we are and how we interact and create our realities with others" (Cunliffe, 2003, 989-990).

Cunliffe's "construction of radical reflexivity" has many parallels to the type of reflexive approach that is taken in this thesis, and in the atlas project itself. Key aspects of this approach include the following: 
[...] questioning our intellectual suppositions; recognizing research is a symmetrical and reflexive narrative, a number of 'Participant' stories which interconnect in some way; examining and exploring researcher/participant relationships and their impact on knowledge; acknowledging the constitutive nature of our research conversations; constructing 'emerging practical theories' rather than objective truths; exposing the situated nature of accounts through narrative circularity; [and ] focusing on life and research as a process of becoming rather than already established truth (990).

In "Processes of reflectivity - Knowledge construction in qualitative research", Adital Ben-Ari1 and Guy Enosh consider reflective processes in terms of both "state of mind" and "active engagement" and identify four levels of reflection: "observation; informants' accounts; text deliberation; and contextualization and reconstruction": "Within this context, reflective processes may refer to deliberate awareness involving both a contemplative stance (state of mind) and intentional activity aimed at recognizing differentness and generating knowledge (active engagement)" (2010, 152).

Sonja Klinsky together with Renée Sieber and Thom Meredith (2008) define reflexivity in the context of a participatory mapping project aimed at sustainability issues:

Reflexivity is used here to describe the process of reflecting on implicit values, conventions, and contradictions. For some, the utility of the concept of sustainability "lies precisely in the degree to which it brings to the surface contradictions and provides a kind of discursive playing field in which they can be debated" (Robinson 2004, 382). Similarly, O'Riordan and Voisey's (1998) concept of revelation argues that sustainability requires broad public engagement with the basic values that underlie conventional, unsustainable patterns. The process of identifying and grappling with these tensions becomes an important element of effective public engagement. We suggest that reflexivity demands that tools help reveal the values underlying (un)sustainability and encourage reflection on both personal decision making and the social context of such decisions (85).

\subsection{Critical Interpretive Method}


In this thesis, a critical interpretative method is used to make sense of the two-pronged approach that has emerged in the Lake Huron Treaty Atlas-making process. This method combines conceptual analysis with reflexive narrative analysis to describe and explain the various ways that critical academic perspectives have worked together with Anishinaabe perspectives in the design and development of the Atlas. According to the reflexive approach, experiences and conversations associated with the ongoing design and development of the Atlas constitute the evidence upon which the thesis statement is based (Davies, 2012). In a similar fashion, Trena Paulus et al. (2010) report: “Our empirical materials were our own conversations during our collaborative research process" (853).

The entire atlas-making process is replete with collaborative conversations. Moreover, in the transdisciplinary context of the atlas project, the idea of research team has been expanded to include a variety of atlas-making contributors coming from a variety of communities of interest (Pyne and Taylor, 2012; Pulsifer et al. 2011). Due to the fact that the atlas project is the focus of this thesis research, my role as geonarrative and content editor for the Atlas (to be discussed in subsequent chapters) has been central, making a reflexive narrative account of the Atlas-making process appropriate.

\subsection{Reflexive Narrative Analysis}

Chapter 8 introduces the notion of "reflexive tracking" as it follows the reflexive logic of the Travel in the Making of the Atlas Map and tells about many events related to the making of the prototype Treaties Module (phase I of the atlas project). It reflects on the performative nature of the iterative processes and weaves together the story of the atlas-making process, recounting how the project 
to tell the story of the Lake Huron Treaty process evolved and commenting on how the two-pronged approach has worked in the iterative development of the Atlas maps (and the Atlas itself). While chapter 9 tracks the Map of Maps; reflects on some of the editing decisions that were made to create the various maps; and, discusses some of the iterative processes that went into their making, their significance and their relationship to some of the other maps in the Atlas. The reflexive accounts provided in each of these chapters are intended to convey the emergent nature of the iterative processes I was involved in as the geonarrative and content editor for the Atlas.

Oakley (2007) refers to "retrospective narratization", and Davies (2012) refers to "retrospective multi-narrative", to describe an approach to narrative analysis that includes "a description of [the] experience of an investigation which necessitated the use of the first person mode of writing, but also to offer some academic analysis arising out of it" (Davies, 2012, 747). According to Davies:

Narratives can be a positive source of insight, giving an intimate understanding of a life and inquiring into selfhood. Narratives have a plot, a purpose, goal or trajectory, they are interpretive and recollective, often focusing on a discrete phenomenon, and they have a 'teller' who can use the power of emotion in the voicing of the story (Roberts, 2002). Narrative accounts, auto/biographies and ways of writing that 'tell of the telling', allow tensions, nuances, complexities, confusions and unclear thoughts to remain and allow for a movement between intellectual and intuitive or other nonobjective forms of knowing and thinking, between 'distance and proximity' (Game and Metcalfe, 1996, 168) $(2012,747)$.

Davies reflects on the "difficulty of juxtaposing and combining a personal and impersonal analysis in one piece of writing" (2012, 748), and cites Holmes $(2010,148)$ who "has argued for urgent theoretical and empirical attention to the emotionalization of reflexivity, suggesting that our conceptual and empirical tools for looking at emotional life need honing" (Davies, 2012, 748). 


\subsection{Critical Conceptual Analysis}

The conceptual analysis component of this thesis is included throughout, and involves discussions of new concepts that have emerged through the Atlas design and development process, such as "geo-transcription" and "mapping epiphany". It also involves the elaboration of existing concepts, such as "performance", "holism" and "narrative", "development" and "reconciliation".

\subsection{The Reflexive Relationship between the Thesis and the Atlas}

This thesis extends the two-pronged reflexive approach that has emerged through the iterative development of the Atlas by combining conceptual analysis with storytelling. From a positionality and reflexivity perspective, many stories are relevant to a full understanding of the genealogy of the Atlas. There is always at least one story behind any story; however, for the purposes of this thesis, a selection of illustrative examples will be discussed.

The Lake Huron Treaty Atlas is the performative 'product' of a wide variety of interactions and discoveries along a variety of dimensions, in a number of spaces. A significant part of 'the story' of this atlas project goes beyond the online 'product' itself. The stories about the making of the Atlas are a part of the atlasmaking process, and therefore a part of the Atlas. The many stories comprising the Lake Huron Treaty 'story' are always in-the-making, sometimes inspiring new stories, other times attracting people to add to them. In these ways, the Atlas is a living entity, with the digital product being a complement to face-to-face interactions and other relational exchanges between people; relationships between people and the land; relationships between people and institutions; and 
relationships between people and ideas, as conveyed in books and other texts, for example.

One important interaction concerns the relationship between this thesis and the Atlas. The question has come up time and time again throughout the course of the atlas project concerning the relationship between the dissertation and the online atlas project. The short answer it is that the thesis is a part of the Atlas and the Atlas is a part of the thesis. That is, they exist in a mutually interdependent, holistic and reflexive relationship with one another. The way in which the Atlas and the thesis are two sides of the same coin can be better understood by applying the conceptual adaptions made by Denis Wood and John Fels (2008) to Gerard Genette's (1997) concepts of "paratext" and "epitext" to describe and explain the relationship between a text and the writings that surround it. Inspired by Genette's conceptual distinctions, Wood and Fels (2008) introduced concept of the "epimap" to describe and explain written works surrounding maps, and built on Jane Camerini's (1993) earlier work on the relationship between texts and maps. According to Wood and Fels, "The epimap consists of the discourse circulating around a map, designed to shape its reception: advertisements, letters to reviewers, endorsements, lectures, articles, and so on ... the epimap work[s] to position the map in a certain way and to lend to it the authority to do work in the world" (2008, 211-12).

It is not only the thesis, however, that exists in a mutually interdependent relationship with the Atlas-making process, but all of the papers, funding proposals and presentations that have been prepared and written about the atlas project (and the processes that have contributed to the generation of those 
'products'). This thesis relates to these other writings in a reflexive manner in the way it comments on their role in the iterative development of the Atlas.

Understanding this thesis and the other written works revolving around the Atlas as constitutive elements of the Atlas draws attention to the holistic nature of the mutually interdependent relationship between the Atlas and the written works that surround it. A colleague of mine recently commented in an email exchange that reading the published papers about the Atlas brought her experience with its maps to life, enriching their educational value for her (Dr. Tracey Lauriault, September 15, 2012, personal communication). An important function of the thesis ties in with this reflective observation. Only part of the 'atlas story' was told in each of the papers published between 2009 and 2012, which 'tracked' the Atlas-making process in an as-it-happens fashion, and provide a reflective record of the project's evolution - both theoretically and practically.

The thesis adds a new layer of reflexivity to the atlas project by reflexively tracking the Travels in the Making of the Atlas Map and the Map of Maps. It folds in the previous thoughts, observations, conceptual insights and reports on the atlas project, picking up where previous commentaries left off and carrying insights further. The thesis acts reflexively in both a forward and backward looking direction, blending discussions of potential atlas capabilities with considerations of events in the past such as the iterative processes that gave rise to the emergence of the atlas maps, and the Atlas itself.

3.7 Reflexivity and the Two-pronged approach to the Lake Huron Treaty Atlas - Concluding Remarks 
The two-pronged approach to incorporating methodological perspectives from complementary knowledge traditions has allowed for the engagement of different perspectives in a variety of mapping processes. Johnson, Louis and Pramono (2006) introduce a two-pronged approach in their critical Indigenous approach to cartography: "Indigenous communities must take a two-pronged approach toward implementing mapping projects: on the one hand becoming literate in these cartographic methods, while on the other hand developing a critical consciousness that attends to the dangers that accompany the use of modern cartographic technologies" (83). It was around the time that I was reading this paper that I started to use the phrase "two-pronged" approach to describe the method that was emerging in phase I of the atlas project, although I had forgotten that I had first read the phrase in this paper. Therefore, I attribute this phrase to the original authors; although, in this case Anishinaabe perspectives comprise one prong and critical academic perspectives, the other. With this said, these two prongs are not mutually exclusive categories, and there is increasingly considerable overlap between the two. As a result of this, although I have continued to refer to the two-pronged approach to atlas design and development, since May 2011 I have also begun to refer to the braided approach to better reflect the intersections between knowledge communities (broadly construed) whose perspectives have contributed to the ongoing atlas project.

The next chapter will provide a brief overview of some significant aspects of one strand of critical academic thinking in this braid, critical cartography, a discipline that is looking at the past in new ways, as it reconsiders what is beneath its feet and how to map it - at the same time that it asks itself: "What is a map?" 


\section{Part II}

\section{Re-visioning Cartography in A Growing Community}

Part II provides a review of some of the critical cartographic and critical Indigenous cartographic literature with a focus on literature that has been relevant in the Atlas design and development processes.

The critical cartographic literature is vast (see for example, Crampton, 2001; Kitchin and Dodge, 2007; Pearce, 2008; Turnbull, 2007; Corner, 1999; Cosgrove, 2005; Harmon, 2003; Monmonier, 2006, 2004, 1995, 1991; 1992; Caquard, 2011). It includes participatory mapping (Fox et al., 2005; Parker, 2006; Irwin et al., 2009;) and extends to the rapidly expanding field of critical GIS and participatory GIS.16 Despite differences in thematic focus and the extent to which the various practitioners in critical cartography engage in either "theory" or "practice", critical cartography tends to be a reflexive practice (Cunliffe, 2003), emphasizing either deconstruction (Harley, 1988, 1989, 2001) or reconstruction (Pearce, 2008), or both (Caquard et al., 2009; Pyne and Taylor, 2012).

A specific intersecting branch of the broader category of critical cartography is critical Indigenous cartographies, which aim to incorporate Indigenous ways in mapping practices in a manner that both enriches these

\footnotetext{
${ }^{16}$ Although the following important examples of work in the area of PPGIS are not discussed in this part, they are included in the bibliography for reference: Wright et al. (1997); Schuurman (2001 and 2000); Schuurman, and Kwan (2004); Sheppard (1995); Sieber (2007 and 2006); Talen (2000); Aitken (2002); Aitken and Michel (1995); Carver (2003); Carver et al. (2001); Couclelis (2004 and 1996); Craig and Elwood (1998); Elwood (2008, and 2002a and b); Elwood and Ghose (2004); Elwood and Leitner (2003); Elwood and Leitner (1998); Ghose (2001); Ghose and Elwood (2003); Jankowski and Nyerges (2003); Jankowski and Nyerges (2001); Jarvis and Spearman (1995); and Krygier (2002).
} 
practices, and avoids the pitfalls associated with misappropriation and misrepresentation of Indigenous perspectives, knowledges and ways. ${ }^{17}$

Evident throughout the literature reviewed in this part is a clear dissatisfaction with conventional status quo assumptions and ways, in favour of approaches that are more closely aligned with Indigenous perspectives and understandings. It is this sort of tendency that signals a readiness on the part of the critical cartographic (and broader critical academic) community to be open to learning from and incorporating Indigenous ways in its work.

${ }^{17}$ Although the following important examples of work in the area of critical Indigenous cartographies are not discussed in this part, they are included in the bibliography for reference: Wainwright and Bryan (2009); Baldwin et al. (2004); Barnes and Duncan (1992); Chambers et al. (2004); Laituri and Harvey (1995); Lewis (1998); Poole, (1995); Roth (2009); Rundstrom (1993 and 1991). 


\section{The Relational Space Turn to Performance: Critical Perspectives in Cartography}

It has been said that cartography is in the midst of an ontological crisis (Kitchin and Dodge, 2007), meaning that it is in the midst of a radical transformation away from a focus on representation, communication and objectivity (Crampton, 2001) and toward a focus on performance, reflexivity and narrative - in short, toward a relational approach to understanding (Crampton, 2001; Kitchin and Dodge, 2007; Pearce, 2008; Turnbull, 2007). The new moral consciousness that attends this transformation is characterized by an overarching preoccupation with promoting justice. This includes a focus on enhancement of agency and empowerment, an important aspect of development, as discussed in chapter two. The emergence of new "critical" ways of thinking about and "doing" maps has become especially relevant to those peoples who have historically been silenced or misrepresented by colonial maps (Brealey, 1995; Peluso, 1995; Sparke, 1998, 2005) - which actually turns out to be quite a diversity of people. The emergence of critical approaches to cartography, combined with technological advancements, has led to a mass questioning of the map as a neutral, objective representation of reality, and has extended to questions concerning the map's status as an object as well.

The past 25 years has seen a proliferation of writing in the area of critical cartography. This review focuses on authors whose work has played a formative role in the design and development of the Lake Huron Treaty Atlas, which has in turn given back to critical cartography by providing examples of - sometimes abstract - critical cartographic concepts. In Brauen et al. (2011), we provide the following summary of the emerging trends in cartography: 
In recent years, critical approaches to cartography (Wood and Fels, 1986; Harley, 1989; Pickles, 1995a and b; Crampton and Krygier, 2006) have considered the political nature of maps and have looked at mapping as a process (Turnbull, 2007), both in terms of the design and development of maps and in terms of their use. These approaches have attended to information that historically has been omitted from conventional—often colonial—maps and atlases and have expanded the scope of possible map themes to include all manner of social issues (e.g., wellness, sexual orientation, personal experience, shared interests). The increasing prevalence of participatory mapping projects indicates a shift toward understanding processes of mapping as vehicles that can strive for social justice and inclusion, depending on the ethics and intentions of those participating in the processes. Indigenous cartographies provide one example of participatory mapping that engages in such critical, alternative approaches to maps and geography in general (Johnson, Louis and Pramono, 2006; Louis, 2007; Palmer, 2009, 2012; Pearce, 2008) (27-28).

Brian Harley was a forerunner in the development of a deconstructionist view of maps that emphasized the importance of knowing who created the maps, the motivations for creating maps and their uses (Harley, 1988, 1989). Following Harley, authors in the critical cartographic tradition began questioning the ontological status of maps as objects. Taking a broader view, they focused on maps as performances and began to develop concepts such as mapping practices (Perkins, 2003; Perkins and Dodge, 2009) and emergent mapping (Turnbull, 2007).

There are many ways to group the various approaches to critical cartography. This chapter begins with a summary of the move to deconstruction in cartography and proceeds to a brief review of the relational space literature since it is relevant to understanding the critical approach to cartography envisioned by a growing number of cartographers. Critical reflexive approaches in all areas generally question status quo assumptions (Cunliffe, 2003), and critical cartography is no different. Considering the age old association between 
cartography and "the land", with its focus on mapping spaces and places, it is no wonder that critical cartographers concern themselves with questioning cartographic assumptions related to "space". Many critical cartographers reject binary distinctions and, exercising a broad perspective, many advocate a relational view of space (Del Casino and Hanna, 2006). This has led to new approaches to emergent mapping (Turnbull, 2007), and projects to create maps as narratives of experience (Kwan, 2007; Pearce, 2008), which will be discussed in turn. The proliferation of community mapping activities in this millennium, both in the paper and the digital mapping contexts, has evolved hand-in-hand with a growing literature in participatory mapping, participatory GIS and volunteered geographic information, and will be discussed under the theme of cartography and community. This chapter concludes with a discussion of the political economic significance of critical approaches to cartography.

\subsection{Cartography as Deconstruction}

Critical deconstruction of maps is pursued in order to draw attention to the relationship between maps and various forms of power. Part of this concern involves drawing attention to the information that is "selected out" in the process of mapmaking, and the intentions and understandings of the mapmakers themselves. A frequent example is the interpretation of colonial settlement (and other) maps as instruments of conquest and administrative control (Caquard et al., 2009). 


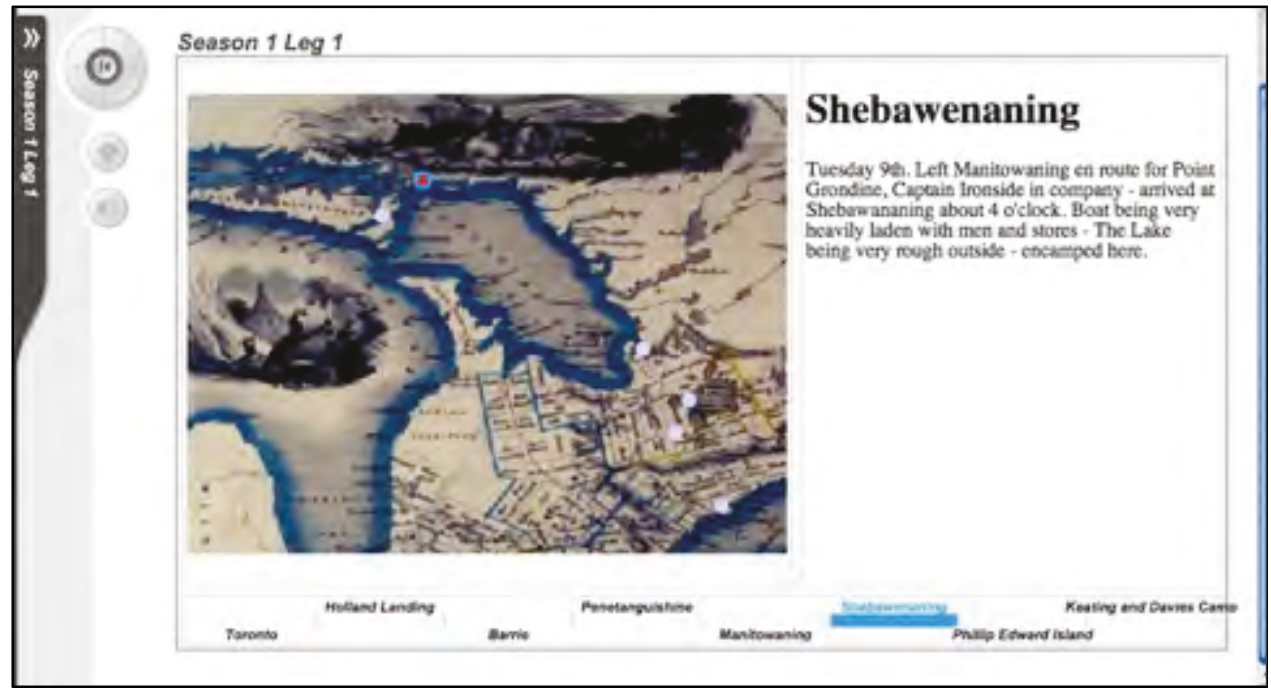

Figure 4.1. Screenshot showing 1849 Settlement Map in the Treaties Module of the Atlas of Indigenous Perspectives and Knowledge as background for Surveyor Dennis's journey out of "organized territory", and into "unorganized" unsettled Anishinaabe Territories; illustrating both deconstructive and constructive reflexive - approaches to critical cartography.

Brian Harley is generally acknowledged as the father of critical cartography for his efforts to point out the socially constructed dimensions of maps; and their role, use and interpretation, specifically their role in mediating colonialism. It has also been pointed out that Harley did not go all the way in terms of elaborating a new critical approach to cartography but that he may have eventually done so, had it not been for his untimely passing in December 1991 (Edney, 2005).

Deconstructionist approaches to cartography highlight the ideological "force" of maps. Matthew Sparke (1998) points out the "recursive proleptic effects of mapping - the way maps contribute to the construction of spaces that later they seem only to represent" (466). Referring to Richard Helgerson's (1992) work on the emergence of state cartography in Elizabethan England, Sparke provides an example of this influence: 
Helgerson, Camden, and others were part and parcel of a civil and conceptual revolution in which it was made to seem as if the land itself spoke of the kingdom as a single state. Consequently, he claims: "The cartographic representation of England did have an ideological effect. It strengthened the sense of both local and national identity at the expense of an identity based on dynastic loyalty.... Maps thus opened up a conceptual gap between the land and its ruler, a gap that would eventually span battlefields" $(1992,114)$ (Sparke, 1998, 466).

Following Harley, more and more critical cartographers began to question the ontological status of maps (and mappings) themselves (see for example, Crampton, 2001; Kitchin and Dodge, 2007; Pearce, 2008; Turnbull, 2007).

\subsection{The Spatial Turn to Performance in Critical Cartography}

In this new age, maps are increasingly being viewed in a performative and non-representational manner (del Casino and Hanna, 2006). This means seeing maps as being continually in the making. Every time a map user "uses" a map, they are remaking it according to their own contextual needs, abilities and interpretations. As a result of work in critical cartography, instead of being used solely as a means to exert authority over territory and people's understanding of it, mapping is increasingly being thought of as a means to contribute to "human [and world] flourishing" (ul Haq, 1995; Sen, 1999) through the enhancement of agency and the inclusion of multiple - often previously silenced or underattended to - perspectives and ways. The performative turn in cartography includes an emphasis on emergent knowledge, on rethinking and re-doing mapping, often with a focus on journeys and experience. Critical cartographers generally resist rationalism (Turnbull, 2000) and question conventional approaches to space.

My first introduction to a performative approach to cartography was a paper by Vincent Del Casino Jr. and Stephen Hanna (2006), which had been 
assigned to me early in 2007 as part of my geography core course readings. Still months away from beginning to work on the cybercartographic atlas project, I was interested in the way the authors described the possibility of a critical, nonbinary and performative approach to mapping. A brief review of the spatial turn to "relational space" in critical geography will provide the basis for understanding the kind of space that many critical cartographers are mapping, and why their approaches to mapping are so different from the conventional notions of cartography that many are used to.

How does thinking about space relationally differ from conventional conceptions of space? Critical economic geographer Nancy Ettlinger summarizes the contrast well:

Unlike "location," which is a Cartesian matter of latitudinal/longitudinal fixes and related patterns, spatiality concerns processes that occur across space and over time, and are integrally related to social relations - not by cause and effect (e.g. action has spatial effect) but rather by being inextricably bound up in one another. Thus understanding social relations requires understanding processes of the space economy and vice versa (2004, 29-30).

Answering this question in more detail involves looking at the significance of a relational space approach for re-thinking conceptions of the global and the local, of space and place. It is a re-thinking of "space" that has political implications at a variety of levels, which tends to remain unacknowledged unless thought through a relational space perspective.

Perhaps the most important aspect of the relational space perspective is its non-essentialist rejection of binary thinking, especially with respect to the global and the local, space and place. According to this view, space is as real as place (Massey, 2004) and not some abstract, ethereal entity. Further, place is not a 
territorially predefined entity that somehow determines the identities of its inhabitants (Amin, 2004), but is rather an entity that derives its identity through the negotiations of a heterogeneous set of inhabitants, each of which has an evolving and unique set of proximate network relations, in addition to a unique set of more distant network relations (Amin, 2004; Massey, 2004).

Doreen Massey remarks on the link between relational space and the relational nature of identity:

Thinking space relationally, in the way we mean it here, has of course been bound up with a wider set of reconceptualisations. In particular it has been bound up with a significant refiguring of the nature of identity. There is widespread argument these days that, in one way or another, identities are 'relational'. That, for instance, we do not have our beings and then go out and interact, but that to a disputed but none-the-less significant extent our beings, our identities, are constituted in and through those engagements, those practices of interaction. Identities are forged in and through relations (which include non-relations, absences and hiatuses). In consequence they are not rooted or static, but mutable ongoing productions $(2004,5)$.

In her non-essentialist construal of individuals, Massey maintains, “We do not have our beings and then go out and interact". This view acknowledges the way that people - as relational individuals - are always "in the making", which opens up possibilities for considering personal transformation and growth as a function of relationships. Paradoxically, it would seem that it is through a relational perspective that we are able to appreciate the uniqueness of places and people.

Massey reinforces the notion that there is no pre-given categorical identity associated with any scale (say, the global or the local). This is because the same relational logic is applied to both space and to place:

If space is a product of practices, trajectories, interrelations, if we make space through interactions at all levels, from the (so-called) 
local to the (so-called) global, then those spatial identities such as places, regions, nations, and the local and the global, must be forged in this relational way too, as internally complex, essentially unboundable in any absolute sense, and inevitably historically changing (Massey, 2004, 5).

It is important to point out briefly how this rethinking of space opens up the discussion of any space-even intrapersonal in the sense that each person can be said to have a relationship with him or herself. In philosophy of mind, intrapersonal space is studied under the guise of 'intentionality'; we are inherently self-reflective or reflexive creatures. Incorporating such a relational approach to space is essential for critical cartographic approaches that aim to map experience (see for example, Kwan, 2007).

Although spaces and places are relationally constructed and not a priori givens, scale or dimensionality does matter, as do the relations between scales. We inhabit a multidimensional world as we interact in ideational spaces, institutional spaces, and interpersonal (and intrapersonal) spaces, for example. In this context, words written down on paper and lines drawn on maps influence attitudes and behavior in ways that people sometimes fail to fully - or even partially - recognize.

Erik Swyngedouw has argued for the ontological priority not of the global nor of the local, but of process:

... scale is not and can never be the starting point for sociospatial theory. Therefore, the kernel of the problem is theorizing and understanding process. According ontological priority to a processbased view takes the focus away from both the "global" and the "local" as the starting point for descriptive and explanatory analysis. It refuses to tackle global-local relationships in terms of dialectic, an interaction or other mode of relating a priori defined things $(1997,141)$. 
Instead "scalar spatial configurations" are produced through processes that involve sociospatial dynamics: "The theoretical and political priority, therefore, never resides in a particular geographical scale, but rather in the process through which particular scales become reconstituted" (Swyngedouw, 1997, 141). This process-based and relational view of space is contrasted with approaches to geography of scale that assign "motive, force, and action to pregiven geographical configurations and their interaction rather than to struggles between individuals and social groups through whose actions and their nested articulations become produced as temporary standoffs in a perpetual transformative sociospatial power struggle" (Swyngedouw, 1997, 141). Despite the fact that Swyngedouw focuses on struggles and does not mention more positive, mutually supportive and co-operative interactions, his process view of the scales of relational space, which focuses on interactions between people, leads us to an analytical focus on the ways in which relationships and interactions reflect scale and define spaces.

The basic ontology of the relational space perspective sees places as inherently political nodes of negotiation or contestation. It is a process-oriented view in which places are constituted through encounters. The basic ontology of an emotionally mediated relational space view (which I worked to clarify in my $\mathrm{PhD}$ core course studies) extends the relational space ontology to include a concern with understanding the dynamics of the actual encounters. It assumes (i) the basic ontological fact of people as thinking-feeling creatures, (ii) that people's thoughts and feelings interact in ways that are subject to standards of moral rationality, and (iii), that those thoughts and feelings shape the moral conduct and outcomes of negotiations and contestations that comprise relational places. 
In this context, it is important to have a language or conceptual framework with which to understand this human geographical microspace (Thrift, 2004a); and in this regard, people like Mei-Po Kwan (2007) have begun to try mapping these microspaces of experience.

A relational view of space puts the emphasis on performance. Nigel Thrift employs non-representational theory to understand performance. The geography that non-representational theory is concerned with is the space of microsocial interaction: "This is the space of everyday social life, a flow of responsive and relational activities that are joint, practical-moral and situated in character and constitute a new understanding 'of the third kind'"(Thrift, 1996, 14). Thrift refers to nonrepresentational theory as a "loose" theoretical framework rooted in four "obsessions": (i) time-space consciousness, (ii) the sensuousness of practice; (iii) the subject (the between-ness of joint action); and (iv), agency (action-oriented, through attitudinal and motivational dimensions). The main driving force behind these dimensions is a common concern with discovering previously unacknowledged modes of existence and finding ways to describe and understand them.

A non-representational theory approach takes an emergent and dynamic approach to understanding the world, where society is viewed as a relatively durable network of heterogeneous actors ... who are able to produce more or less durable moments of forging connections through the exercise of expressive power" $(1996,15)$.

Although there are a number of other characteristics to specify, enough should have been said to demonstrate the difficulty of picturing nonrepresentational theory. In part, because the ontology, epistemology and morality 
of non-representational theory are so different from the commonly internalized Cartesian view of human beings, it takes great effort to conceptualize it. This is also true of other concepts involved in critical academic discussions of performativity. Although both the concept and the word, "performativity" itself tend to be nebulous, they have nevertheless set the stage for projects seeking to concretize understandings of performance.

In their guest editorial to the Environment and Planning $A$ (special issue on performance), Alan Latham and David Conradson (2003) acknowledge the history of geography's interest in studying practices and experience, pointing to people like Hagerstrand $(1975,1976,1982)$, and others from "the more interesting strands of humanistic geography (Buttimer, 1976; Buttimer and Seamon, 1980; Ley and Samuels, 1978; Tuan, 1977)" (1901). At the same time, they point out two significant turns in geography that have led to the renewed interest in practice:

First, the turn towards culture in human geography has seen - both in the vigour of contemporary cultural geography and in a more general willingness to explore culturally inflected styles of analysis and interpretation - has helped move questions of social practice closer to the intellectual centre of the discipline. Second, this renewed interest in practice - in combination with an engagement with a diverse collection of social-theoretic traditions that have, to use John-David Dewsbury's words (2003, page 1908), nudged us into recognising that "this is a world in which the metaphysical referent for truth is now in doubt [...] accompanied by a growing appreciation of the limitations inherent in many established approaches to social practice (1901-1902).

Together these turns, which are linked to the focus on space as a relational entity, have provided a supportive environment for the emergence of performative approaches to cartography. A performative perspective of maps as processes, including those related to their making and their use, "goes beyond 
seeing the map as an immutable and neutral physical object" (Pyne and Taylor, 2012, 95), and rests on two central assumptions according to David Turnbull:

One is that meaning, understanding and knowledge are based in embodied practices. The other is that the performance of knowledge practices and their attendant knowledge spaces and artefacts simultaneously structure and shape our sociocultural world in a process of coproduction. We make our world in the process of moving through and knowing it" $(2007,142)$.

In "Maps, mapping, modernity: Art and cartography in the twentieth century", Denis Cosgrove suggests "an examination of art and cartography in the twentieth century should focus on mapping practices rather than on maps as such":

While the history of cartography has freed itself from debilitating debates over the scientific and artistic status of maps, considerations of the relationship between art and cartography have continued to focus largely on pre-modern maps, avoiding critical examination of twentieth-century art and science in cartography and leaving intact the impression that these followed distinct paths in the modern period. In this paper, however, I have drawn on theoretical work in Science Studies and taken account of modern art's separation from aesthetics to suggest that an examination of art and cartography in the twentieth century should focus on mapping practices rather than on maps as such. A summary overview of modern-art movements and selected works indicates a continued, if critical, engagement of avant-garde artists with cartography, and the examination of more popular newspaper artwork produced in the context of the intensely modern visual culture of mid-twentieth century Los Angeles indicates a similarly close connection between modernity, art and cartography (2005, 35).

Del Casino and Hanna (2006) approach the process of mapping from a performative perspective that includes the practices involved in "making" the map and the intellectual-emotional acts of interpreting maps each time they are perceived. For them, a map is more than just ink on paper or a digital display on the computer screen: It is the processes of meaning-making that comprise its making and re-making. 
The approach to maps as practices is consistent with the "ontogenetic" conception of mapping practices put forward by Robert Kitchin and Martin Dodge (2007) who "propose a radical departure in ontological thinking concerning maps: a shift from ontology (how things are) to ontogenesis (how things become), or from the nature of maps to the practices of mapping ... they are not ontologically secure representations, but, rather, a set of unfolding practices" (Kitchin, 2008, 213). In contrast with the view that maps are the results of carefully laid out plans and theories, several critical cartographers take an emergent approach to mapping that emphasizes their exploratory nature. In Pyne and Taylor (2012) we summarize some of these perspectives:

David Turnbull $(2007,142)$ recommends a hodological approach to mapping: "In geography, hodology is the study of paths, in philosophy, the study of interconnected ideas, and in neuroscience, the study of the patterns of connections in the white matter of the brain ... It is the hodological emphasis on the concept of trails that is central to a performative understanding of the co-production of knowledge and space". Rob Kitchin and Martin Dodge (2007) have a complementary understanding that goes further than those of Jeremy Crampton (2003) and John Pickles (2004) in its view of the map as continually in the making, held to any sense of ontological security only by the shared assumptions and the knowledge and mapping tasks that people bring to it. A hodological approach to the map-making process sees mapping both as trail making, in the sense of knowledge creation, and as trail following, in the sense of tracking the emerging knowledge that results from a series of knowledge-sharing interactions (97).

Emphasizing the trail-making function of maps is relevant to critical Indigenous cartographies, which will be discussed in the next chapter.

Kitchin and Dodge (2007), Turnbull (2007) and others cast the critical cartographic project as one that involves "rethinking knowing and mapping where the key questions relate to the similarities and differences in the ways space, time and movement are performed and to how those similarities and 
differences are handled" (Turnbull 2007, 141). Along the same theme, Turnbull advocates creating "a third space, a space in which the possibilities of agonistic pluralism can occur based on a performative rethinking of knowing and mapping" $(2007,141-42)$.

According to Rob Kitchin and Martin Dodge:

Maps emerge in process through a diverse set of practices. Given that practices are an ongoing series of events, it follows that maps are constantly in a state of becoming; they are ontogenetic (emergent) in nature. Maps have no ontological security, they are of-the-moment; transitory, fleeting, contingent, relational and context-dependent. They are never fully formed and their work is never complete $(2007,340)$.

For Jeremy Crampton (2009) maps can be both "part" of performance the performance itself, and Denis Cosgrove (2008) sees maps as cultural practices. Both authors discuss community mapping in their discussions of performative cartography, revealing the interconnectedness of these themes.

\subsection{Cartography and Community}

The wake of the digital age has seen an increase in community mapping projects, which can provide fruitful contexts for collaborative work involving different perspectives. An important purpose of community mapping is to "ground truth" policy by involving community input into sustainability and other issues. There are many issues related to the democratization of cartography. These include concerns regarding the need to educate people about maps and mapping techniques if they are going to be engaged in cartographic practices (Johnson et. al, 2006; Donna Williams, personal communication August $8,2008)$.

In "Participatory mapping as a tool for capturing local perspectives on cultural landscape: A case study of Ostlänken", Chia Jung Wu and Karolina 
Isaksson contrast participatory and scientific maps: "Participatory maps are distinguished from scientific maps ... to emphasize that the contents of the maps derive from a local perspective rather than a scientific perspective" $(2008,7)$. With this said, taking a broad view of community, it is important to neither reify, nor to exclude the scientific perspective from participatory mapping exercises.

Two main dimensions of participatory mapping can be identified along a continuum. An 'informative' online participatory map involves the map user having an interactive relationship with the map. This type of mapping is participatory in the way it involves the map user as an information seeker. Active online participatory maps involve the participation of the map user in the creation of the map. Active participatory mapping often involves more 'on the ground' map creation work, and almost always includes the informative dimension as well. Wiki-type informative maps come the closest to combining the informative and active dimensions to the extent that they allow the user to both use and contribute to the further development of the map.

In "Constructing community through maps? Power and praxis in community mapping" (2006), Brenda Parker takes an alternative view to the common perception that Indigenous peoples are under-represented in the critical cartographic practice (which is testament to the growing interest in Indigenous mapping). Parker "links critical cartography literature to goals of communitymapping practitioners through themes of inclusion, transparency, and empowerment" and "highlights the social construction and practice of 'community', the relationship between maps and power, the definitional difficulties associated with community mapping, and the figurative and literal boundaries that constrain community cartography" (470). 
Drawing together art and cartography through a method they use refer to as "a/r/tography", Irwin et al. (2009) begin to map, explore, reveal and understand the rhizomatic nature of the relational identities that comprise the particular city of Richmond, British Columbia. They follow Mei-Po Kwan (2002), who "insists that the term 'site' needs to be re/imagined beyond a particular location to 'sites' that are not geographically bound, but rather, are informed by context. This relational understanding is constituted through social, economic, cultural and political processes"' (Irwin et al., 2009, 64).

In "Art, maps and cybercartography: Stimulating reflexivity among mapusers" (2005), Sebastien Caquard and Fraser Taylor make the case "for encouraging reflexivity by making map-users aware that any map is a 'construction of the image of space' and is thus inherently subjective":

Informing map-users of the constructed dimension of maps is particularly vital given the exponential production of maps via the Internet. This growth expands the presence of potentially dogmatic and misleading messages ... On the one hand cartographers have widely used the aesthetic dimension of art to increase the impression of objectivity associated with maps and on the other hand, developments in the humanities provide grounds to challenge this concept of map objectivity. In this context the potential of cybercartography to combine multiple media, art, technologies, and perspectives into maps is highlighted. This chapter concludes by arguing for stronger presence of artistic creativity and social criticism in maps on the Internet to stimulate map reflexivity (285-6).

Taylor and Pyne (2010) describe how the evolving Lake Huron Treaty Atlas "brings cybercartography more fully into the realm of public participatory GIS (PPGIS), especially with respect to its preoccupation with collaborative investigation involving a diverse range or 'community' of participants, each with a unique and distinct manner and form of contribution" (12). 


\subsection{Maps as Narratives of Experience}

In "Framing the Days: Place and Narrative in Cartography", Margaret Wickens Pearce contrasts those who reject the use of geospatial technologies in favour of mapping practices (such as sketch mapping), that are "personal and centered on the exploration of emotional meanings in the landscape" $(2008,18)$ with others who "engage in alternative mapping practices that assume a critical stance toward geospatial technologies and the cartographic traditions from which they are derived (Johnson, Louis, and Pramono 2006; Pearce 2008; Pickles 2004; Turnbull 2007)" (Caquard et al., 2009, 84). Pearce notes the recentness with which alternative epistemologies have begun to be incorporated into contemporary geospatial technologies and seeks through her work on "Framing the Days", "to contribute to [the] re-imagination of the capabilities of Western geospatial technologies by expanding cartographic language for expression of place without leaving the realm of the digital map" $(2008,18)$. In her mapped narrative of a canoe journey, Pearce includes "personal trajectories of experience across the digital map using GPS" (Pearce, 2008, 17) in her attempt "to express the geographies of human experience and place in the map" and to give “experience its own layer" (Pearce 2008, 20).

Incorporating personal narratives into maps is becoming more and more common. The purpose of personal mapping is to bring to light previously "unmapped" information concerning people's experiences in relation to their

environments. The inclusion of personal maps can be relevant in community mapping projects. Examples include Kwan (2007), Parks (2001), and del Casino and Hanna (2006). 
Paying attention to the multiple dimensions in which relationships occur, Taylor and Pyne (2010) describe the potential for geonarratives to convey interscalar relationships, ranging from those that exist between individuals and institutions to those that comprise the atlas-making process itself (12). In a complementary approach to broadening the concepts of "map" and "Atlas", Wood and Fels (2008) (discussed in chapter three) extend the map to include all of the text surrounding its interpretation, presentation and publication. Surrounding publications reflect the authors' experience with the map, and provide the opportunity for reflexive interpretation of the processes that went into the making of a map, drawing attention to its form and content as well.

\subsection{Critical Cartography and Policy - Concluding Remarks}

In "An introduction to critical cartography" (2006), Jeremy W. Crampton and John Krygier characterize critical cartography as:

[...] a one-two punch of new mapping practices and theoretical critique. Critical cartography challenges academic cartography by linking geographic knowledge with power, and thus is political. Although contemporary critical cartography rose to prominence in the 1990s, we argue that it can only be understood in the historical context of the development of the cartographic discipline more generally. We sketch some of the history of this development, and show that critiques have continually accompanied the discipline. In the post-war period cartography underwent a significant solidification as a science, while at the same time other mapping practices (particularly artistic experimentation with spatial representation) were occurring. Coupled with the resurgence of theoretical critiques during the 1990s, these developments serve to question the relevance of the discipline of cartography at a time when mapping is increasingly prevalent and vital (11-12).

Whether a deconstructive, a performative, a community, or a narrative approach to cartography is taken - or a combination of all four - critical cartographers all seem to agree - either implicitly or explicitly - on the "power of maps" to influence the way people look at and approach the world. With this 
awareness, critical cartographers try through their work to use that power for good, to demystify the colonial power implicit in maps and to engage in participatory and collaborative mapping projects that give voice to a diverse range of people. At the same time maps and new cartographic technologies are being used extensively in governance and warfare, resource exploration and development, and planning and construction. These uses affect everyone; a critical understanding of these uses is necessary as well.

I agree with John Pickles (2004) and Margaret Wickens Pearce (2008) that conventional cartographic representations often fail to adequately reflect and communicate experience, sense of place, and diversity in world views in a nondominatory manner (Caquard et al., 2009; Pickles, 2004); and, I acknowledge the emerging trend in critical cartography to think about new ways to re-engage with maps and mapmaking" (Herb et al., 2009, 332)", where the focus is not only on deconstruction but also on reconstruction (Herb et al., 2009; Kitchin and Dodge, 2007; Pearce, 2008; Turnbull, 2007; Wood and Fels, 2008; Pyne and Taylor, 2012). In this context, the new and exciting sub-discipline of critical Indigenous cartographies has emerged in recent years. There are many similarities and overlaps in the literature between what I have termed "critical Indigenous cartographies" and the "mainstream" critical cartography reviewed in this chapter, including their interest in such concepts as experience, performance, and community. Perhaps most important, is their acknowledgement of the political, economic, social, cultural and individual relevance of maps and mapping, and their desire to participate in cartography in a way that contributes to good political, economic, social, cultural and individual change, toward reconciliation involving development as Bimaadiziwin. 


\section{5. $\quad$ Adding Voice to Mapped Silences: Critical Indigenous Cartographies}

"There is an Indigenous Geography in the making - a new approach to land consciousness involving map reading and map-making that is leading to the establishment of an encompassing, innovative and pragmatic new discipline" (Jose Barreiro, 2004).

“On 13 September 2007, the United Nations General Assembly adopted the Declaration on the Rights of Indigenous Peoples, recognizing a desire on the part of Indigenous peoples globally to reassert self-determination over their land, natural, and cultural resources. Indigenous academics and allies from many disciplines have brought their skills to bear on securing land and resource rights. Arguably the greatest impact has come from cartographers and those with cartographic skills, particularly in the effort to legitimize land and resource claims by Indigenous communities. Also significant has been the work of those who critique the engagement of cartographers working within Indigenous communities, with the primary concern of avoiding more significant losses than gains in this engagement" (Louis, Johnson, and Pramono, 2012).

When Jose Barreiro wrote the statement nine years ago, Brian Harley had already published "Silences and secrecy: The hidden agenda of cartography in early modern Europe" (1988) and "Deconstructing the map" (1989), followed by John Pickles with Ground Truth: The Social Implications of Geographical Information Systems (1995a). Bridges have been forming for quite some time in the world of cartography. Concerns with mapping such things as experience, emotion, art and Indigenous perspectives are intertwined. When Renee Pualani Louis, Jay T. Johnson, and Albertus Hadi Pramono (2012) wrote their statement just last year, the field of research in the area of critical and critical Indigenous cartographies had expanded immensely. However, despite this progress, these authors also noted:

[...] much of the academic dialogue about this work has appeared in non-cartographic journals, such as the recent special issue of Cultural Geographies (vol. 16, no. 2) titled "Indigenous Cartographies" (Sletto 2009). More than a decade has passed since Cartographica published Monograph 44, Social and Cultural 
Cartography, which brought to the forefront issues of Indigenous peoples and cartography (77).

The critical cartography literature is full of conceptual transformational thinking (for example, Kitchin and Dodge, 2007; Perkins, 2003; Crampton 2001, 2009; Turnbull, 2007; Pearce, 2008). Being on the edge of a novel epistemological and ontological stance with respect to maps and mapping, many of the discussions in this literature are either lacking in exemplary detail or are calling on others to begin mapping from this new epistemological and ontological perspective.

David Turnbull (2007) has stated that the best way to engage in a critical approach to mapping - especially in an intercultural context - is to engage incommensurable perspectives in a dialogical tension with one another in a nondominatory manner (Pickles, 2004), where western epistemologies and ontologies do not take priority or precedence over Indigenous understandings and ways of approaching the world.

This chapter provides a review of literature under the guise of the 'land as map' theme in order to broaden the discussion sufficiently to take the focus off of a strictly "western" perspective of maps and mapping. In this section I combine Anishinaabe and critical cartography conceptual themes that focus on relationships to the land and the implications of this for cartography. This chapter looks next at the colonial history of mapping relationships and mapping encounters between the Original Peoples of Turtle Island and the settlers from a historical deconstructive cartography perspective, and then discusses some emerging trends in critical Indigenous cartographies. Presenting critical Indigenous cartographies in its own chapter does not deny the intricate inter- 
relationships between this stream and the broader critical cartographic literature, but provides the opportunity to consider this area of critical cartography in more depth than the other approaches have received in the previous chapter.

\subsection{The Land as Map}

In that Empire, the craft of Cartography attained such Perfection that the Map of a Single province covered the space of an entire City, and the Map of the Empire itself an entire Province. In the course of Time, these Extensive maps were found somehow wanting, and so the College of Cartographers evolved a Map of the Empire that was of the same Scale as the Empire and that coincided with it point for point. Less attentive to the Study of Cartography, succeeding Generations came to judge a map of such Magnitude cumbersome, and not without Irreverence, they abandoned it to the Rigours of sun and Rain. In the western Deserts, tattered fragments of the Map are still to be found, sheltering an occasional Beast or beggar; in the whole Nation, no other relic is left of the Discipline of Geography (from "Travels of Praiseworthy Men" (1658) by J.A. Suarez Miranda, a fictional reference in Jorge Luis Borges, $A$ Universal History of Infamy, 1972, 131, quoted in Turnbull, 1993, 2).

This introductory quote in David Turnbull's Maps are Territories: Science is

an Atlas (1993) brings up the issue of the ontological assumptions people bring to "maps" and "mapping":

Those who are imbued with what is sometimes called 'the Western world view' think of objects as having fixed characteristics and defined boundaries ... and as having a position specifiable by spatial co-ordinates ... It may well be that Western ontology is in part reinforced by the centrality of maps in Western thinking and culture. Therefore, because of this possible circularity, one must be careful not to take one's own view as definitive of all maps.

There are notoriously problematic issues, as well as unexplored ones, bound up in such questions as 'What is the relationship between the map and the territory?' and 'When is a map not a map but a picture?' Many of these problems are reflected in the apparent cogency of Korzybski's dictum 'The map is not the territory' (Science and Sanity, 1941, p. 58). After all, if the map were identical with the territory it would literally be the territory. It would have a scale of an inch to an inch and, apart from anything else, it would be unworkable as a map since you would have to be standing on it or in it. Lewis Carroll described such a map in Sylvie and Bruno concluded. In this fantasy, a Professor explains how his country's cartographers experimented with ever larger maps until 
they finally made one with a scale of a mile to a mile. 'it has never been spread out, yet', he says. 'The farmers objected: they said it would cover the whole country, and shut out the sunlight! So now we use the country itself, as its own map, and I assure you it does nearly as well.' (3).

I had the opportunity to reflect on the quotation cited by David Turnbull, and on Turnbull's comments on it, as I sat on my 22nd floor balcony looking south over the city. The skyline view of the city is an expansive one. From that perspective, I was able to see the Dunton Tower at Carleton University to the south, which reminded me of the time I was completing my Master's thesis in an office on the 22nd floor of that building in the fall of 2005. Doing a rough count of the street blocks between where I sat and the university, I estimated it would take me about 40 minutes to walk there. If it was a nice day, and I took my time, it might take a little longer. As my gaze continued across the city, I remembered other events in my life associated with places I could see with my bird's eye view. The words, "the land IS the map" started circulating through my thoughts as I began reflecting on the extent to which the 'living' city-as-map I was looking at was actually based on a multitude of plans and maps: The houses, apartment buildings and roads, all based on blueprints. At the same time, I thought about the emergent nature of the city over time, and how the precedents established by some plans, for example road construction survey plans, continued to have an effect on contemporary plans for the city that lay beneath me.

The Original Peoples of Turtle Island used the land as a map for the stars when they constructed mounds to relate to the stellar constellations. Often the knowledge sources for how to construct these mounds and where to locate them were dreams. It is important to note how the earth map of the stellar 
constellations would be a small scale map that would nevertheless give rise to the experience of being "in" the map.

The Anishinaabek made pictographs to tell stories on the land. These pictographs were made with ochre, and had deep historical and spiritual significance. They also made and used wampum belts as maps. For example, linked closely with the Seven Fires Prophecy is the Anishinaabe migration story, which has followed the prophecies that were inscribed into the belt. Their movement has been tracked, both by significant markers inherent in the landscape and by the "discoveries" of anthropologists (see Belfy, 2011 for examples).18

David Woodward and G. Malcolm Lewis (2000) distinguish between cognitive, performance and material cartography, pointing out how little remains of Indigenous material cartography prior to contact with Europeans. One of the reasons for this, of course, is that the newcomers destroyed Indigenous cartographic artifacts as they moved across Turtle Island. The mounds provide a good example of this. Wampum belts were destroyed as well. With this said, David Turnbull's observation regarding current knowledge about the construction of the Chartres, referred to in chapter 6, applies equally to the question of pre-contact "evidence" of Indigenous cartographies: “... absence of evidence is not evidence of absence" $(2000,62)$.

Woodward and Lewis (2000) refer to the lack of a cross-cultural consensus on the meanings of "map" and "cartography" and point out the Eurocentric

\footnotetext{
${ }^{18}$ An initial sketch of the Anishinaabe migration story, which is linked to the Seven Fires Prophecy, is mapped in the Language and Culture Map of the Lake Huron Treaty Atlas.
} 
nature of "the search for 'maps' in these cultures, particularly when accompanied by the idea that maps privilege the societies in which they are found ..." (1).

In Masons, Tricksters and Cartographers, David Turnbull (2000) reminds his readers that Europeans did not always use maps to understand and relate to the world:

Important questions in understanding the formation of our technoscientific world are when, where and how plans and maps started to play a formative role in shaping our modes of understanding. The auxiliary question asks when plans and maps became synonymous with scientific understanding, thereby obscuring the messy practices that underlie them (53-4).

In "Cartographic Calculations of Territory" (2010), Jeremy Crampton questions western European assumptions of territory that underlie colonialism. These assumptions have had particular relevance in the context of treaty interpretation and implementation between the British Crown and the Anishinaabek, for example, and reflect deeper concepts, including those concerning people's relationship to the land. In the case of the Lake Huron Treaty, the Anishinaabek in the 1850s often referred to the European concept of distance in terms of 'days travelled', including information regarding the means of travel, the pace of travel and the type of travelling party. Thus, speaking maps to one another and including detailed information was a commonplace activity for the Anishinaabek. Imagine how crude a descriptor the Anishinaabe must have found in the European concepts such as 'league' and 'mile' to describe travel across the land and by water when they began encountering Europeans and entering into treaty negotiations with the British Crown. 
Getting at the question of the ontologies of mapping, Renee Pualani Louis cites Rundstrom (1995), who in turn draws on Paul Connerton's (1989) distinction between incorporating and inscribing cultures:

Incorporating cultures traditionally emphasize oral communication and other performance-based modes (e.g., dance, painting) in transmitting all sorts of meaningful information. The actions, lasting hours or days, carry greater meaning than any object they produce. In contrast, inscribing cultures hold and fix meaningful information years after humans have stopped informing, and typically must do so by means of some object (e.g., maps, GIS). Storage is crucial, and leads to stasis and fixity (Rundstrom, 1995, 51, quoted in Louis, 2004, 9).

According to Louis,

[s]uch an incorporating culture can be found in Hawaiian cartography. Like the Maori, pre-contact Hawaiians had a clear understanding of the world they lived in and communicated their perception of the world orally (Kelly, 1999, 1). Hawaiian cartographers privilege process by incorporating their understanding of their island setting into their mo' olelo (stories), oli (chant), 'ö lelo no'eau (proverbs), hula (dance), mele (song) and their mo'o kü 'auhau (genealogy). This is a form of cartography categorized by Woodward and Lewis (1998) as "performance or ritual cartography" and may "take the form of a nonmaterial oral, visual, or kinesthetic social act [in order] to define or explain spatial knowledge or practice" $(4)(2004,9)$.

Margot Beyersdorff's (2007) account of the Andean-Spanish encounter between 1577 and 1586, tells about how the Andean way of mapping 'led the way' in the joint mapping venture, which was expressed via the mediation of Spanish cartographic technology. In doing so, she identifies a pre-contact Andean mapping methodology:

The adjudication of entitlements was conducted by both Spanish and native Andean authorities who walked a course of topographical features and constructed markers that bound a pueblo de indios, or township. The Spanish conceived this practice, known as amojonamiento, as a lineal pathway in order to inspect, place, or repair mojones, or cairns. On the other hand, native Andeans envisioned the reconnaissance of mojones and natural features, or saywa, as a circular route known as muyuriy (130). 
Another example, this time of an Algonquin Anishinaabe a teaching that has been passed down through oral tradition since time immemorial, well $\ldots$ at least for as long as the pike has been around. Algonquin Anishinaabe Elder Jacob Wawatie (Mowegun) began speaking about the pike head teaching with me in 2009. Briefly, each bone in the pike's skull reflects a different individual and role in the community, according to each individual's gifts: their interests and unique perspectives and their skills and abilities. Jacob referred to this as the basis of the Anishinaabe constitution for his Nation (personal communication, January 3, 2013). And then there is the cartography of Jacob Wawatie, which he has "performed" numerous times in the telling of the story of the "Turtle Island and the Elephant", passed down to him through his grandparents and to them from theirs, ... As Jacob tells the story, he illustrates the geography of the Turtle on paper and reinterprets conventional western European geomorphology in Anishinaabe terms.

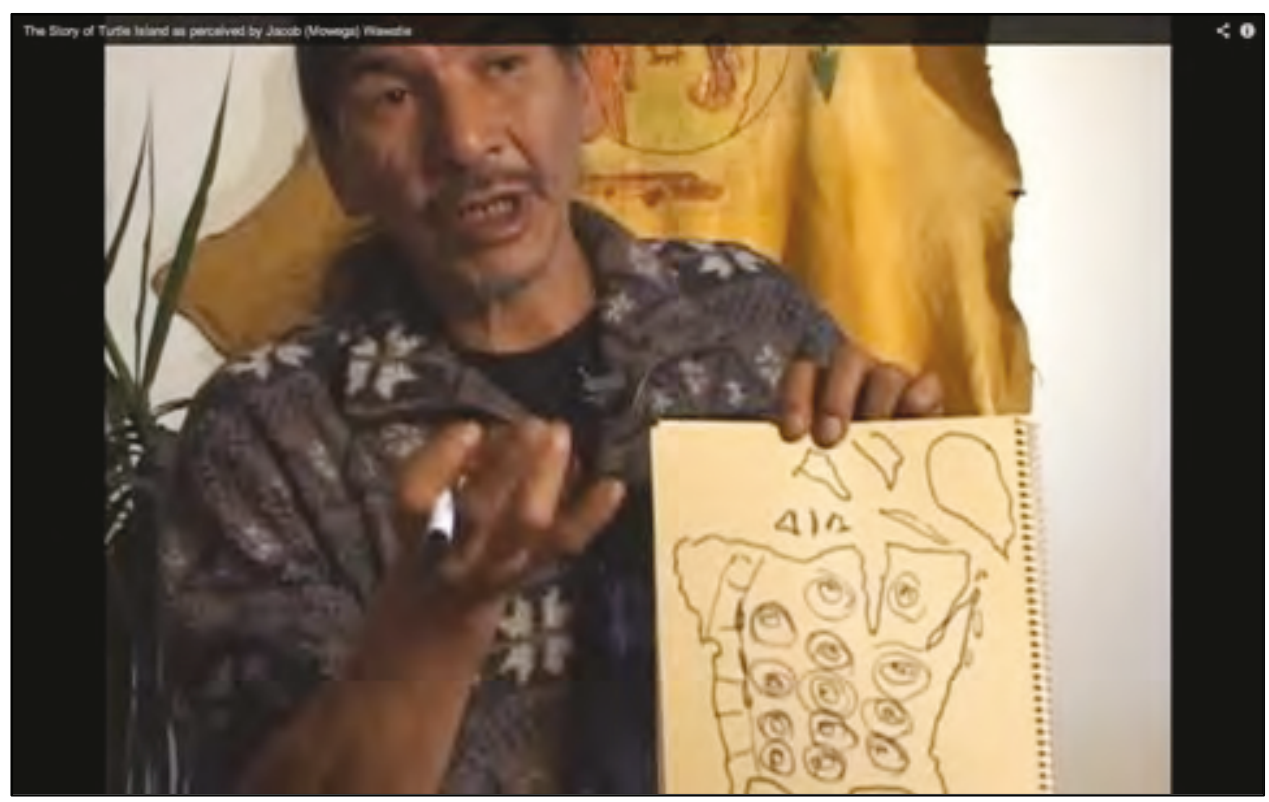


Figure 5.1. Screenshot showing Jacob Wawatie teaching through the Turtle Island and the Elephant map story, which touches on many dimensions of existence on Mother Earth. ${ }^{19}$

The conventional western assumption that the past is dead does not factor into this mapping that is Jacob's story. In fact, the intergenerational wisdom that is alive in this story makes the question of whether or not there was a pre-contact Indigenous concept of cartography immaterial. Coming back to David Turnbull's apt statement, " ... absence of evidence is not evidence of absence" (2000, 62), it is not a matter of "evidence", but of having the capacity to perceive that will allow us to begin to answer questions we may have about Indigenous cartography.

\subsection{Early Colonial Mapping Encounters}

By the time of early contact, the Original Peoples of Turtle Island were already learning about western spatial communication practices - and tailoring their mapping practices to European understandings. In order for them to be able to communicate their geographical knowledge to the newcomers, they first had to understand how the newcomers viewed cartography. Having such a diverse population across Turtle Island, with a vast trade network, the Original Peoples were already adept at translation and interpretation. As a result, they were able to successfully impart knowledge about the land to the newcomers and to learn the newcomer's cartographic trade at the same time. The converse cannot be said of the newcomers. Although they were successful to some extent in understanding the language and ways of the Original Peoples, their narrower epistemological and ontological focus, including their utilitarian and self-

19 The video of Jacob Wawatie's Turtle Island and the Elephant map story is available at http: / / www.youtube.com/ watch?v=u88mfQIhTig. 
interested ethics, led the newcomers to a rather thin and anemic view of the Original Peoples, skewed to suit their particular moral and epistemological persuasions.

Deconstructive historical cartography has contributed immensely to revisioning cartography in a way that draws attention both to the "colonial intention" and to the "Indigenous perspective" in hybrid maps reflecting both western and Indigenous worldviews. When it comes to "the 'bounding' of native territory and the subsequent dispossession of natives from their land", B. Kamanamaikalani Beamer and T. Kaeo Duarte (2008) identify two contrasting patterns:

Cole Harris [2004], for example, writes that 'the management of the dispossession of the colonized of their land rested with a set of disciplinary technologies of which maps, numbers and law were perhaps the most important'. Recent work has, however, qualified such a perspective. Karl Offen [2007], for example, has shown how indigenous spatial practices could contribute to the making of colonial maps. Offen demonstrates how the spatial practices of the Mosquitia between 1629 and 1779 played a part in the creation of Spanish and English maps [that] documented Mosquitia territory. In a very different context, Thongchai Winichakul [1997] suggests that the tools of the 'colonizer' were used by indigenous elites forming the process of creating modern Thailand. Thongchai provides a thoughtful analysis of the creation of a non-European independent state in order to resist colonization, in which indigenous elites adapted some of the processes of European colonization for their own means. A recent article by Brett L. Walker [2007] on the Japanese mapping of Sakhalin Island also provides an example of non-European usage of mapping technologies for the protection and expansion of national sovereignty. Japanese agents such as Mamiya Rinzo appropriated European mapping technologies which position Sakhalin island into a system of representation recognizable to those knowledgeable of European cartographic principles (70).

In their own historical study, Kamanamaikalani and Duarte (2008) focus on the Hawaiian surveys, referred to as the Mahele of 1848, and the successes for 
Hawaiian sovereignty gained by appropriating European knowledge and using it to their own advantage.

Margot Beyersdorff (2007) agrees with Kamanamaikalani Beamer and Duarte (2008) in her observations of the ways in which Andean pre-contact cartographies shone through "the chorographic view of landscape in Spanish cartography" [...] "when both traditions converged upon the implementation of the Crown mandated surveys known as the 'Relaciones Geográficas del Peru' $(1577-1586) ":$

These mappings and accounts display the native Andeans' adaptation of precontact place identification practices to Spanish boundary marking methods. The mappings demonstrate a hybrid cartographic art, which combines manuscript and block print within the pictorial format. In one of the maps the artist-scribes include a relación in Quechua (and its Spanish version), a rare example of this genre in Andean letters. Indigenous cabildo authorities, exemplified by Felipe Guaman Poma de Ayala, adopted writing practices by recording place names. This activity led to a pan-Andean lexicon of toponymns for judicial purposes (129).

\subsection{Mapping with the Newcomers in a Reconciliation Context}

In our introduction to "A "Living' Atlas for Geospatial Storytelling: The Cybercartographic Atlas of Indigenous Perspectives and Knowledge of the Great Lakes Region" (Caquard et al., 2009), we summarize the past and current relationships between Indigenous peoples and "scientific" maps:

The relationship between Indigenous communities and maps is often ambivalent. Historically, scientific maps have been used by nation-states primarily to assert their territorial rights and reinforce their power over Indigenous and other communities. In contrast to this practice, various alternative maps have more recently become "a vehicle of resistance, a language whereby rights to place may be asserted or through which non-dominatory representations might be cultivated" (Pickles 2004, 113).

$$
\text { [...] }
$$


The importance of Indigenous communities mapping and representing their own territories and environment has been widely acknowledged. Domains such as counter-mapping (Peluso 1995). contrapuntal cartography (Sparke 1998), community mapping, and participatory GIS (Sieber 2004) have helped communities to reclaim their sovereignty over lands (Herlihy and Knapp 2003), with more or less success, depending in some cases on how mapping was conducted (Tobias, 2000). The appropriation of cartographic techniques and technologies such as GPS or GIS has proven to be very efficient and useful for this purpose. However, these techniques remain technologically complex and do not necessarily allow for the non-dominatory representation of unique understandings of space, environment, and culture (84).

The practice of using the oppressor's tools for emancipatory purposes is not a new one. For example, in War Under Heaven (2002),20 an excellent account of the Pontiac Wars, Gregory Evans Dowd tells about how the Anishinaabek used the British maritime warfare strategy they had learned successfully in battles against the British.

In Pyne and Taylor (2012), we summarize the main challenges faced by contemporary cartography in relation to Indigenous peoples:

When it comes to engaging with mapping in a way that involves Indigenous peoples, the challenges [of] "remapping" include maintaining an ongoing attentiveness to avoiding misappropriations of knowledge, understandings, and perspectives: "The problem that faces Indigenous peoples worldwide is to find a way to incorporate Western [geospatial technologies] and cartographic multimedia while minimizing the mistranslations, recolonizations, and assimilations of conventional technoscience" (Pearce and Louis, 2007, 123). A primary critical concern is whether or not cartography is capable of meaningfully conveying such things as experience, Indigenous perspectives and knowledge, and critical academic approaches to the status quo (Johnson et al., 2006, 2007; Johnson and Murton 2007; Palmer 2009, 2012; Palmer et al., 2009; Turnbull 2007) (93-4).

${ }^{20}$ Chief Shining Turtle from Wigwaasminising (Whitefish River First Nation, Reserve No. 4) recommended War under Heaven to me in February 2008. According to Chief Shining Turtle, in order to more fully understand the significance of the Royal Proclamation, it is necessary to be familiar with the historical geography that preceded it. 
The idea of the map is broadening as more and more Indigenous communities are engaging in participatory approaches to mapping that include counter-mapping (Peluso, 1995) and participatory GIS (Sieber, 2004). However, with the emergence of this trend has come the need for critical cartographic literacy in order to avoid the pitfall of misrepresentation. The project of encouraging "critical cartographic literacy" (Johnson et al., 2006), involves encouraging awareness of the ways in which Western cartography remains rooted in the same Cartesian-Newtonian epistemology that underpins the colonial worldview:

To engage the technologies of Western cartography is to involve our communities and their knowledge systems with a science implicated in the European colonial endeavour and is a decision which should be made only after examining not only our past experiences of colonial mapping/surveying but also the long history of Western cartographic traditions (Johnson et al., 2006, 82).

As we pointed out in Pyne and Taylor (2012):

Johnson et al. (2006) refer to the Wet'suwet'sen and Gitxan's use of maps in court proceedings as an example of a situation in which Western technologies were beneficial "in establishing Indigenous connection to lands, resources and cultural sites." While this is true, it is important to remember that these maps combined Western cartographic knowledge with traditional knowledge. The maps were presented in court, but in addition, ceremonies were performed and arguments were made based on maps that reflected a Wet'suwet'sen and Gitxan worldview (Sparke 1998, 2005). The extent to which traditional knowledge and ways went along not only with the composition but also with the presentation of the maps reflects an important counter to the hegemony of Western cartographic tradition associated with colonialism, and provides evidence that a significant degree of critical cartographic literacy was operating in that case (88).

The critical cartographic consciousness achieved by the Wet'suwet'sen and Gitxan involved the ability to integrate traditional knowledge and culture with western cartographic technologies in a manner that ultimately contributed to the 
Supreme Court's recognition of the admissibility of orally transmitted knowledge in the court proceedings (Sparke, 2005). The beneficial effects for Indigenous peoples of understanding the nature and history of western cartographic tools and techniques include increased empowerment and agency (Johnson et al., 2006), especially when it comes to participating in mapping projects.

Julie Taylor (2008) describes "the advent of Indigenous countermapping" among South African San groups. Peter Pulsifer, Gita Laidler (now Lubicjc), Fraser Taylor and Amos Hayes (2011) describe their work to create an "Indigenist data management program": “The International Polar Year (2007-2008) (IPY) established an IPY data policy that guides a formal data management (DM) process. The DM system envisioned includes data based on Indigenous knowledge systems linked to data collected in the Western scientific tradition" (108).

In "Power of Maps: (Counter) Mapping for Conservation", published in a special issue of the online Acme Journal, Leila Harris and Helen Hazen (2006) discuss the benefits of incorporating Indigenous perspectives into conservationoriented counter mapping initiatives:

In the conservation realm, there is increasing attention to the possibility that technologies such as GIS-central to the 'Western' or 'scientific' conservation planning toolkit-further cleave distinctions between 'expert' and 'lay' or 'indigenous' knowledges, providing just one example of how mapping practices can reinforce common socio-political exclusions. In response to such potential, efforts to forge more just conservation futures are focusing increasingly on democratized mapping, and the incorporation of alternative knowledges in GIS or cartographic forms, or valuation of alternative mapping practices (106). 
Harris and Hazen (2006) note the preference for permeable fencing in Kenya, a strategy that has resulted from incorporating Indigenous knowledge regarding landscape permeability into the mapping process:

[...] there has been a move, led by Richard Leakey, to encourage the fencing of protected areas in certain situations. This is intended both to prevent wildlife leaving the security of protected areas and to protect local people from the costs of crop-raiding. Lake Nakuru National Park in Kenya is now completely surrounded by electric fencing (Leakey, 2002). Permeability refers primarily to landscape characteristics that allow movements of animals. Here, rather than preserving tracts of habitat, species mobility is the overriding goal. Already the permeability concept has been influential in informing freeway redesign and urban redevelopment efforts, and is a primary focus of David Foreman's Rewilding Institute [...] Many conservation organizations are thus now reorienting strategies away from protected areas to wider focus on issues such as landscape permeability (112).

In “Mapping Dreams/Dreaming Maps: Bridging Indigenous and Western Geographical Knowledge", Irene Hirt describes her work as a cartographer in a Mapuche counter-mapping and participatory mapping project in southern Chile from 2004 to 2006. Hirt notes how "dreams and dreaming practices are integrated into knowledge-building processes in many Indigenous societies, and therefore represent a source of geographical and cartographic information“ $(2012,105)$.

Mapping dreams and dreaming practices expands the cartographic limits of "Indigenous territorial dimensions - such as the sacred and the spiritual, as well as the presence of non-human actors"' (Hirt, 2012, 105).

Hirt's work is evidence of non-Indigenous cartographic researchers taking dreams seriously as basis for knowledge, and incorporating them into their work. This is not so different from the legendary Rene Descartes whose epiphany "I think, therefore I am" was the result of a series of dreams, a fact he was adamant to have recorded by his biographers (Withers, 2008); an ironic twist considering 
Descartes' status as the father of rationality, a "discipline" based on logic and deduction sourced in known "observables" in the 'conscious' world, certainly not in dreams!

\subsection{Links to Critical Cartography - Concluding Remarks}

A number of alternative mapping approaches have emerged in critical Indigenous cartographies with the purpose of regaining sovereignty in many areas, including how people understand their history and how they relate to themselves, others and their environment. Critical cartographers seek to rethink and redo mapping by acknowledging and attempting to transcend a colonial past (Kitchin and Dodge, 2007; Pickles, 2004; Turnbull, 2007) in which maps were used by nation-states primarily to assert their territorial claims and impose an administrative architecture on the land. Today, mapping practices are increasingly being engaged in as solutions to complex social and economic challenges. The movement began with an emphasis on decolonizing history by Brian Harley who focused on deconstructing colonial maps to understand the ways they were used to exercise and promote colonial authority $(1989,2001)$. Over time critical cartographers began to look more deeply into the ontological status of maps themselves. They came to view maps as processes that give rise to emergent knowledge and are never complete (Kitchin and Dodge, 2007). They considered epistemological issues as well, such as overcoming incommensurability in the mapping Indigenous perspectives and knowledge (Pyne and Taylor, 2012; Turnbull, 2007). 


\section{Part III}

\section{Developing an Atlas for Development}

Part III examines cybercartography as the central approach that frames the atlas project (chapter 6). In addition to providing a description of the atlas project that highlights its cybercartographic characteristics, this part provides an overview of the iterative processes that comprise the atlas project (chapter 7 ). The central theme that ties these two chapters together is the concept of development. The emergent, iterative approach taken to atlas development is linked to its ability - through collaborative participation - to foster human development through shared experiences, and teaching and learning through transdisciplinary interactions. 


\section{Cybercartography: A Fruitful Framework for Growing an Atlas Vision}

Cybercartography has been inspiring and supporting the development of a diverse yet related family of atlas projects since 1991 when Dr. Fraser Taylor received a grant and established the Geomatics and Cartographic Research Centre. Beginning with a focus on use and usability issues in the development of the first cybercartographic atlas prototypes, the Cybercartographic Atlas of Antarctica and The Cybercartographic Atlas of Canada's Trade with the World, the focus shifted almost concurrently with the launch of these atlases in 2006 to a 'mapping with Indigenous peoples' focus. This shift would lead to further developments in cybercartography, marked by the addition of "six additional features" to Fraser Taylor's original "seven key elements of cybercartography (Taylor, 2003).

In the evolution of Fraser Taylor's distinctive approach to Web 2.0 cartography, he has participated in 'on the ground' development work, put forward definitions of cartography and provided the theoretical and methodological framework for a growing number of cybercartographic atlas projects, involving an increasingly diverse range of collaborators in the process of telling stories with maps.

This chapter will consider the nature and significance of cybercartography as a conceptual and applied framework for the construction of cybercartographic atlases in the context of a broader discussion concerning the nature of maps and atlases. Following this general introduction, a summary of the cybercartographic Lake Huron Treaty Atlas will be given, drawing attention to the mutually 
interdependent relationships between the Atlas and its cybercartographic framework.

\subsection{Defining the Map and Cartography}

In 1968, Arthur H. Robinson took a functional approach to defining the map: "The primary function of a map is to serve as a reduction of all or part of the earth's surface for the purpose of recording, presenting, or analysing the spatial positions and the interrelationships of phenomena occurring thereon" (quoted in Sills, 1968). Brian Harley and David Woodward put forward a more explicitly social definition in their History of Cartography, Volume I (1987, xvi). Conscious of the need to identify and break free of colonial intentions behind maps, Harley and Woodward's definition focused on the epistemological value of maps to enable broader "spatial understandings" of processes and events in the "human" world beyond the "earth's surface". While their definition may be overly narrow in terms of its ontological focus on maps as "graphic representations", it does specify that maps apply to the "human world", and draws attention to the social function and context of maps.

Fraser Taylor (1991) combined Robinson's interest in the function of maps with the social concerns of Harley and Woodward (1987). Recognizing the new digital context of maps and mapping in 1991, Taylor' definition embedded the map firmly in the context of the discipline of cartography and emphasized the importance of the processes by which maps are created:

The map is central to cartography and thus appears explicitly in my own definition of the discipline: "The organisation, presentation, communication and utilisation of geo-information in graphic, digital or tactile form. It can include all stages from data presentation to end use in the creation of maps and related spatial information products." I do not believe it is wise to define 
cartography without referring to the map, the process by which it is created, and the social context in which it exists $(1991,214)$.

According to David Akerman (1991), "the emergence of the atlas form is related to the upsurge of Western European interest in geography and cartography during the sixteenth century", and "once the atlas idea developed, atlas structure reflected the growth of a "national geographical consciousness" (54). If this is the case, then an analogous "international geographical consciousness" is with us today. The proliferation of online mapping activities and the ease and speed with which this trend has developed, suggests this is indeed the case. As Taylor (1991) predicted:

The 1990s will see the already substantial impact of digital technologies on cartography continue to accelerate. Digital cartography played a vital role in the Gulf War and GIS applications are exploding in a wide variety of application areas. The scientific technology of cartography is important but in my view the issues, which should determine new directions for cartography are not primarily technical. Indeed, it can be argued that if the prevailing technological paradigm is allowed to predominate then much will be lost. To allow our understanding and definition of cartography to be determined by a technological imperative would be a mistake.

In 1984, at the ICA meeting in Perth, the term 'A New Cartography' was introduced to draw attention to the dramatic impact of information technologies on the discipline. Part of the concluding argument was that "The greatest ... challenge facing cartography does not lie in teaching or learning new techniques, but in creating a radically new concept for our discipline" (22).

Soon after his call for "a radically new concept for our discipline" in 1991, Taylor introduced the idea of the cybercartographic approach to Atlas making, a community approach to gathering and sharing knowledge involving the ongoing creation and use of an emergent web-based geospatial platform. 


\subsection{Why an Atlas? Why not just a Map?}

It is no doubt due to his "on the ground" experience with the multiple dimensions of social, political and economic development processes (and their interrelationships) through his early development work in Kenya, that Taylor realized the atlas context was required in order to 'reflect' multiple dimensions through cartographic practices; a single map would just not do. What was needed was a vehicle to tell different stories cartographically, one that reflected the multiple dimensions of "social and economic processes" and the interrelationships between them. What was needed was an Atlas.

The most obvious characteristic of cybercartography is that it is about atlases. The significance of cybercartography as an atlas making-rather than a mere map-making framework cannot be understated. An atlas ties together a collection of maps with a narrative logic that contributes to understanding and interpretation of both itself and the maps (Akerman, 1993; Wood and Fels, 2008). As a compendium, it is both comprised of its maps, and about them. In his $\mathrm{PhD}$ thesis, "On the shoulders of a titan: Viewing the world of the past in atlas structure", James Akerman comments on the form and social nature of Atlases. According to Akerman,

Gerard Mercator was the first to name a set of maps after Atlas, who in legend was first a Titan, among the fathers of the gods, then a Mauritanian king who had a passion for cosmography, and finally, an astronomer to who whom the first celestial globe is ascribed. It was the image of the god doomed to hold heaven and earth aloft that became the favored model for the frontispieces of Mercator's Dutch successors ... but Mercator, the astronomer and mathematician, geographer and historian, was more intrigued by the "earthly" Atlas deeds:

My purepose then is to followe this Atlas, a man so excelling in erudition, humanitie, and wisedom, (as from a lofty watch tower) to contemplate Cosmographie, as much as my 
strength and abilitie will permit mee, to see if peradventure, by my diligence, I may finde out some truths in things yet unknowne, which may serve to the studie of wisedom. And as the world conteyneth the number of all things, the species, order, harmonie, proportion, vertues and effects; so beginning from the creation: I will number the parts thereof, so farre as methodical reason requireth, according to the order of the creation, and will contemplate physically, that the causes of things may be knowne ... Afterward I will handle Coelestiall things in their ranke: then the Astronomicks .... Fourthly, treat of things Elementarie, \& lastly the Geographicks, and so (as in a mirror) will set before your eyes, the whole world, that in making use of some rudiments, ye may finde out the causes of things, and so by attayning unto wisedom and prudence, by this meanes leade the Reader to higher speculations.

Both the god and king Atlas are essential to Mercator's metaphorical use of "Atlas" as the name for his universal set of maps; for through these new map sets men such as Mercator and his readers were able, in the wake of discoveries and the intellectual upheavals of the Renaissance, to grasp the world once again as the ancient Greeks and their gods had (1991, 1-2).

Akerman presents a founding vision for the "Atlas" as narrative with a broad scope that covers a variety of dimensions. Mercator's inspiration and guiding metaphor for his proposed project to "mirror" 'reality' was none other than the god king himself, Atlas. Mercator's secondary stated purpose, to "set before your eyes, the whole world ... and ... by this meanes leade the Reader to higher speculations" corresponds to the central purpose of both cybercartography and the collaborative project to design and develop the cybercartographic Lake Huron Treaty Atlas: To contribute to an enhanced understanding of relationships between people and between people and the land. In the case of cybercartography, it is the broader goal of achieving a new awareness of social political and economic processes through cartographic narrative; and, in the case of this specific atlas project, it is the more focused goal of enhancing awareness and mutual understanding in Lake Huron Treaty 
relationship processes. This consistency in fundamental goals has made cybercartography an ideal theoretical and methodological framework for the Lake Huron Treaty Atlas, which has involved the emergence of critical, holistic and reflexive mapping practices: seeking, presenting and reflecting knowledge in a way that incorporates Anishinaabe and supporting critical academic perspectives.

\subsection{Cybercartographic Atlas Design and Development}

Fraser Taylor made a long range forecast when he introduced "cybercartography" in 1997:

Cybercartography will see cartography applied to a much wider range of topics than has traditionally been the case... It will also utilize an increasing range of emerging media forms and telecommunication networks such as the Internet and the World Wide Web. It will be a multidimensional cartography using multimedia formats and is more likely to be an integral part of an information package than a stand-alone product.

Cybercartography will also be highly interactive and engage the user in new ways. In organizational terms, it will see new partnerships being created between national mapping organizations, the private sector and educational institutions and the products of cybercartography are likely to be compiled by teams of individuals from very different disciplines and professional perspectives working together (Taylor, 1997, 4).

At this time, cybercartography was at an initial conceptual stage and required further development. In 2003, a year after receiving a \$2.5 million research grant from the Social Sciences and Humanities Research Council for the Cybercartography and the New Economy Project, Taylor released a formal definition of cartography as, "... the organization, presentation, analysis and communication of spatially referenced information on a wide variety of topics of interest and use to society in an interactive, dynamic, multimedia, multi-sensory format with the use of multimedia and multimodal interfaces" (Taylor 2003, 404). 
At the same time, he listed cybercartography's "seven major elements", stating that cybercartography "(1) is multimedia using vision, hearing, touch and eventually smell and taste; (2) uses multimedia formats and new telecommunications technologies such as the World Wide Web; (3) is highly interactive and engages the user in new ways; (4) is applied to a wide range of topics of interest to society, not only to location finding and the physical environment; (5) is not a stand-alone product like the traditional map but part of an information/analytical package; (6) is compiled by teams of individuals from different disciplines; and, (7) involves new research partnerships and the private sector" (Taylor, 2003, 407).

Fraser Taylor has emphasized how intuition and practice preceded the theoretical development of cybercartography. Throughout and after the launch of the cybercartographic Atlas of Antarctica and Atlas of Canada's Trade with the World, theoretical development began to happen in the many areas, for example transdisciplinary research (Lauriault and Taylor, 2005), cybernetics (Martinez and Reyes, 2005) and semantic ontologies (Pulsifer and Taylor, 2005). In addition, Glenn Brauen introduced several innovative sound mapping features for some of the maps in the Atlases that were released under this project (Brauen, 2006).

On April 16, 2008, Taylor shared his six key features with the Geomatics and Cartographic Research Centre team members, and later published them on the Centre's web site. These features are as follows:

1. People use all of their senses in learning. Consequently, cybercartography creates representations that allow them to do this through cybercartographic atlases.

2. People learn in different ways and prefer teaching and learning materials in different formats. Cybercartographic atlases provide people 
with a choice of learning styles or combinations of learning styles. The same information is presented in multiple formats.

3. Effective teaching and learning takes place best when individuals are actively involved and engaged. The multimedia and interactive approaches used in cybercartographic atlases facilitate this.

4. People need the power to create their own narratives, ie. the social computing revolution. The Cybercartographic Atlas Framework provides a mechanism for doing this, which gives some structure and metadata indicating the quality and nature of the narratives that people create. The Framework is also open source and does not require special knowledge in order to create a narrative.

5. Many topics of interest to society are very complex. There is no simple "right" or "wrong" answer to many questions such as global warming and climate change. To understand these complexities different ontologies or narratives on the same topic should be presented in ways that people can easily understand without privileging one over the other. Cybercartographic atlases do this. Of particular importance is giving voices to local people. They can speak for themselves rather than having others speak for them.

6. There has been a shift from "map user" to "map creator" which establishes new forms of democratized teaching and learning. The Cybercartographic Atlas Framework helps to democratize mapping in new ways.

Listing the main characteristics of cybercartography is useful for providing a general idea of the parameters of the approach. However, in my case, it was not until I started practicing cybercartography, which included spending time in the lab and learning by "osmosis", that I began to really understand what cybercartography is all about.

Cybercartography contributes to critical cartography in terms of both theory and application via the iterative development of a growing family of cybercartographic atlases. It is a way of practicing cartography that draws a variety of people into atlas-making projects in a variety of ways. Rooted in Fraser Taylor's early development work (Taylor, 2013), cybercartography is a set of concepts and tools that provides an effective atlas building framework for 
approaching complex social, political and economic phenomena, which include reconciliation processes (Taylor, 1997, 2003, 2005, 2009, 2013; Taylor and Caquard, 2006; Pyne and Taylor, 2012). Providing a space for many perspectives and encouraging people to speak and otherwise express themselves in their own ways is a key ingredient of the deliberative democracy envisioned by the late Iris Marion Young (2000) and a key potential function of cybercartographic atlases.

Another important benefit of the cybercartographic approach to atlas making is the way in which it allows for the development of emergent methods within a general atlas-making framework. As a theoretical and practical atlasmaking framework, cybercartography has fostered a diverse yet related array of atlases since the launch of the cybercartographic Atlas of the Antarctica (Taylor and Caquard, 2006). As with all cybercartographic atlases, the Lake Huron Treaty Atlas is a unique instance of cybercartography that is nevertheless related to the other atlases in a family resemblance manner (Wittgenstein, 1953).

Cybercartographic atlases are transdisciplinary and holistic in nature, with an emphasis on storytelling, knowledge sharing, and enhancing awareness of different perspectives. The name of the software developed to create atlas modules - Nunaliit - illustrates the community orientation of the project. The word nunaliit means "settlement", "community", or "habitat" in Inuktitut, the name given to the dialects of the Inuit language in Canada. This name was given to the cybercartographic framework to emphasize the community based approach that was driving the development of the software in different domains: (1) open specification approaches; (2) modularity; (3) "live" data; (4) geospatial storytelling; and, (5) audio-visual mapping (Caquard et al., 2009). 
Cybercartographic atlases develop over time through a series of iterative processes involving design, implementation and testing phases. Prototypes, both paper and digital, are developed throughout these phases. Discussions of the cartographic possibilities occurring around these prototypes result in alterations in map structure and function over time. Discussions between team members with differing knowledge specializations result in design and development decisions. For example, the iterative development of the Lake Huron Treaty Atlas is partly a function of the relationships and communications between the software design and information infrastructure team and those responsible for the geonarrative content. Although they fall into two relatively distinct knowledge domains, these two groups share intersecting knowledge and responsibilities when it comes to atlas design and development; and, the expansion of knowledge that results from their interactions gives rise to the emergence of maps (Brauen et al., 2011). Since 2006, there has been increasing involvement with Indigenous peoples in iterative atlas-making processes, with a shared interest in overcoming intercultural incommensurability between knowledge perspectives.

Cybercartography is a productive theoretical and methodological framework that is neither too over-specified nor too underspecified. In this respect, the cybercartographic approach to atlas development is similar to Amartya Sen's (1992, 1999) capability approach to human development (introduced in chapter 2). Just as the capability approach (and the related capabilities approach of Martha Nussbaum) has provided the conceptual conditions for the emergence of a wide variety of theoretical and applied approaches to human development (Robeyns, 2003; Crocker, 2006), a growing 
family of mutually inter-related atlases and atlas-related prototypes have emerged within the developing cybercartographic theoretical and methodological framework. These atlases and prototypes are related along a variety of dimensions, including the technological, content, funding/economic, and conceptual dimensions, to be discussed further in chapter 7. The careful balance between theoretical and methodological over- and under-specification makes the cybercartographic atlas framework an ideal context for the emergence of atlases with distinctive character and identity.

As was mentioned earlier in this thesis, a primary concern in this transformational period - for cartography at least - is with whether or not cartography is capable of meaningfully conveying such things as experience, Indigenous perspectives and knowledge, and critical academic approaches to the status quo (Johnson et. al, 2006; Turnbull, 2007). The multimedia, multisensory, multimodal, interactive and/ interdisciplinary nature of the cybercartographic approach to atlas making (Taylor, 1997, 2005; Taylor and Caquard, 2006; Taylor and Pyne, 2010) positions it well to be able to address this concern. Moving beyond traditional representational cartography, the 'living' cybercartographic framework allows for the development of novel geospatial modes of expression that include art and that can be used to better reflect traditional and contemporary Indigenous knowledge and understandings.

Cybercartography takes a broad approach to both science and art, and acknowledges the holistic relationship between science, which includes geospatial technologies, and art, which extends to storytelling. This position guides the design and development phases of cybercartographic atlas projects in the direction of more adequately conveying multiple perspectives. An important 
part of this enabling process consists in the reflexive integration of art with geospatial technologies in order to address the challenges associated with incommensurability of knowledge systems, or multiple ontologies (Turnbull, 2007) in critically innovative and creative ways (Caquard and Taylor, 2005).

\subsection{The Cyber in Cybercartography}

It is true - the term "cybercartography" does have many syllables. While the most immediate connotation that might come to mind probably involves cyber-"space", a more significant connotation is "community". The iterative development of the conceptual and applied dimensions of cybercartography, which began officially in 2003, resulted in the release of the Nunaliit software framework in 2006. The production of this software involved the collaborative participation of a diverse "community" of researchers spanning many disciplines, all sharing the same purpose of designing a community-friendly online, interactive multimedia Atlas. Community interaction in the collaborative atlas-making context involves sharing knowledge and perspectives across disciplines and between cultures. The line between mapmaker and map-user in the making of cybercartographic atlases is definitely blurred.

\subsection{Emerging Development and the Lake Huron Treaty Atlas - Charting New Territory in New Ways using the Cybercartographic Approach}

The research project to create the Lake Huron Treaty Atlas fulfilled my initial thesis goal - to expand the Treaties Module of the Cybercartographic Atlas of Indigenous Perspectives and Knowledge into an Atlas in its own right - by increasing the number of maps from three in the Treaties Module to thirty in the Lake Huron Treaty Atlas. The atlas maps include biography maps; maps related to Anishinaabe language and culture; detailed historical background maps; and 
maps relating to current affairs. The Atlas is now publicly accessible via the World Wide Web, and provides a geographic research and education framework for broader-based public participation with respect to both learning and continuing to "grow the maps" in a distributed and collaborative manner. The ethic of inclusion that is practiced in this project is an important aspect of cybercartography: providing an online cartographic space for Anishinaabe perspectives and sharing knowledge across disciplines and specialty areas. This approach is important for enriching Anishinaabe education, renewing relationships between youth and Elders, reviving traditional culture and language, and fostering intercultural mutual understanding.

The cybercartographic geonarratives in the Lake Huron Treaty Atlas encourage knowledge sharing and critical reflection along a variety of interrelated dimensions: historical, geographical, legal, political, economic, social and cultural. The Atlas has been evolving in an iterative manner since it began in 2007 as a pilot project to create several maps in the Treaties Module of the Cybercartographic Atlas of Indigenous Perspectives and Knowledge. This initial pilot phase of Atlas work was funded by a one-year Inukshuk Wireless grant, and was intended to provide sample content to demonstrate the potential of the cybercartographic approach to tell stories and to reflect Indigenous perspectives in an accessible, interactive and collaborative way. ${ }^{21}$ Subsequently funded by a

\footnotetext{
21 The project to create the cybercartographic Atlas of Indigenous of Perspectives and Knowledge was a joint initiative between the Great Lakes Research Alliance for the Study of Aboriginal Arts and Cultures (GRASAC) and the Geomatics and Cartographic Research Centre with the goals to: (1) create a 'living' atlas that captures the richness and uniqueness of indigenous knowledge from community members and researchers and re-connects it to the communities and their land on an on-going basis. This atlas will serve as a public gateway to some of the material collected by the GRASAC team and will also enable ongoing future community contributions to the content via a web interface on the site. The atlas does not require advanced technical skill to be used and allows community members to input information in their own language through voice input,
} 
three-year 2009-2012 SSHRC grant, the Treaties Module has grown into an Atlas with 30 maps and more potential maps planned, as the result of collaborations with Anishinaabe and non-Anishinaabe community members interested in sharing their perspectives, contributing historical geographical multimedia content, and exposing assumptions implicit in the western worldview (Taylor and Pyne 2010; Pyne and Taylor 2012). Although it is an ongoing project that is always in-the making, the Atlas has been publicly accessible via the World Wide Web since May 2012 as a transdisciplinary research and education tool. The processes involved in knowledge dissemination and facilitating online community-based contributions to the Atlas are the focus of a SSHRC Outreach Grant, which began in the summer of 2012. A significant focus for research under this grant has been directed at improving the technological base for the Atlas to better enable people to log into the Atlas web site, add multimedia content to the existing maps and, in some cases, make maps of their own (Pyne, 2013).

The Lake Huron Treaty Atlas both draws on and contributes to the theory and practice of cybercartography (Taylor, 1997, 2003, 2005, 2009; Taylor and Caquard, 2006; Pyne and Taylor, 2012; Taylor and Pyne, 2010), which advocates a transdisciplinary, iterative and community approach to online interactive Atlas making (Brauen et. al 2011) that involves sharing knowledge across disciplinary and cultural barriers. A good example of this is the way in which taking a cybercartographic approach to geonarrative in the development the Treaties

story telling, video, photographs, documents and other data, as well as connecting this data in new ways to tell a story; and (2) translate this unique knowledge into highly interactive multimedia stories for educational purposes. The transmission of this knowledge is often difficult within the communities, as young people do not always fully appreciate the value of this knowledge in their current environment. New technologies are seen as ways to improve the transmission of knowledge across generations in indigenous communities by engaging young people in new ways. 
Module led to the emergence of a specific form of geonarrative referred to as "geo-transcription" (Caquard et al., 2009), to be discussed further in chapter 8.

Taking a transdisciplinary approach, programming and cartographic information specialists working on the atlas project have learned about the narratives to be mapped in order to become more adept at providing the software basis for realizing those narratives in an online interactive multimedia cartographic context. Similarly, in learning more about the nature, roles and functions of the programming and cartographic information specialists, I have become more aware of the technical limitations and possibilities in my role as the geonarrative and content editor (Brauen et al, 2011).

The atlas project has expanded on the cybercartographic approach through the generation of emerging mapping practices that incorporate Indigenous knowledge and perspectives in new ways. This has involved driving the Atlas design and development process by stories reflecting community involvement instead of technology (Dunn 2007), and applying an emergent method that is consistent with and acknowledges the value of Indigenous methodologies (Tuhiwai Smith, 1999; McCall and Minang, 2005).

While Mercator's aims were noble, maps and Atlases were ultimately used for colonial purposes and came to underwrite and reinforce the colonial vision of territory and administrative territorial control (Akerman, 1991; Harley, 1988, 1989, 2001). It is interesting that the vision for the Lake Huron Treaty Atlas shares Mercator's goal of presenting multiple dimensions in order to raise awareness only this time, it is an awareness of the relational spaces of the Lake Huron Treaty and rejection of the colonial vision that is the goal. 
Throughout the continuing development of the Atlas, the geonarrative focus has shifted and changed; with an increasing number of geonarratives being added over time, and with the ever-present narrative challenge of relating these mapped stories in a manner that reflects a meaningful organizational logic. At the beginning of the atlas project in 2007, the focus was on understanding the treaty-based relationships that occurred in the context of the Lake Huron Treaty investigation, negotiation, signing and survey processes, and that extend to other related historical geographical contexts. The Treaty was signed in 1850 and represented an agreement between the (British) Crown and 17 Anishinaabe communities occupying the North shores of the Lake Huron region. Along with the Robinson Superior Treaty, the Lake Huron Treaty (officially known as the Robinson Huron Treaty) was the first Native-Crown treaty to deal in a "land surrender" fashion with a large landmass and a diverse range of peoples who were nevertheless interrelated by a significant clan system. The atlas project accordingly involves a deconstructionist and collaborative approach to mapping in order to better describe and analyze evolving treaty-based relationships. Together, these approaches involve looking at old maps in new ways by including previously excluded information - excluded not only from maps, but from general knowledge of 'the history' itself. Equally important, the Atlas also employs a "re-constructionist" approach by exploring ways to rethink and re-do maps and mapping in a Anishinaabe way.

The Atlas presents aspects of the Lake Huron Treaty story through a series of interrelated map-based stories or "geonarratives" that provide the basis for critical reflection along a variety of dimensions: historical, geographical, legal, political, economic, social and cultural. In addition to the knowledge and 
perspectives that are being gathered and presented in this web-based multimedia atlas format, increased knowledge and understanding is being shared in ways external to the worldwide web among a growing community of individuals who are interested in participating in the atlas-making process. Thus, the work to produce the Atlas contributes to a growing learning and knowledge-sharing network, providing the basis for future informed discussion and deliberations (Brauen et al, 2011).

Over the past seven years, the iterative development of the cybercartographic Lake Huron Treaty Atlas has given rise to the emergence development-related mapping practices. This affinity with "human development' is rooted in my Master's studies background, combined with the roots established by Fraser Taylor through his development work in Kenya, from which his "development from within" perspective emerged (Taylor, 2013). Accordingly, my approach to cybercartographic atlas making has been a nonhierarchical approach to finding common ground and awareness - the 'get your feet in the mud' approach advocated by Raff Carmen (2000), and discussed in chapter 2.

The collaborative fieldwork component of the atlas project has been an ongoing interactive process of teaching and learning, with a growing web of participants engaging in collaborative meetings and exchanging knowledge in the oral, written and "artistic" traditions. The transdisciplinary, multidimensional and collaborative approach taken in this work has allowed for a rich contextual framework within which to understand treaty-based and other relationship processes, in addition to increased understanding of the context itself. There are many stories to tell about my journey as the narrative and 
content editor of the Atlas; these stories are intertwined with the stories being mapped in the Atlas relaying tracings of the histories of people's relationships to one another and to the land. The main idea behind this "living" atlas project is that there are many stories to tell, in many ways, and they are all related. Although the Atlas stories, or mapped geonarratives have their genesis in the Lake Huron treaty region, the vision for the Atlas is that it will continue to grow, and - like the Spider's web - begin to weave together more and more related stories over time, and across space. There are important links between the stories from all directions, which this atlas project is beginning to gather and share.

Through ongoing work to construct the Governance Paper Trail Map and other maps, the Lake Huron Treaty Atlas has the potential to contribute to theoretical and applied knowledge of the relationships between scales, spheres or domains, most notably the relationship between legal and regulatory institutions and people, their lives and experiences. As a multidimensional project that incorporates a diverse array of interrelated knowledge and perspectives, the project contributes to greater public awareness of treaty history and geography in the broader context of the need for reconciliation in intercultural relations (Bhandar, 2004; Pyne, 2009). As a volunteered geographic information project, it contributes to emerging knowledge concerning web-based participation in a geographic information systems environment, and provides a dynamic example of mapping as a process that goes beyond the view of the map as an object (Kitchin and Dodge, 2007; McCall, 2003; Pyne and Taylor, 2012; Turnbull, 2007). 


\subsection{The Lake Huron Treaty Atlas as a Bridging Tool - Concluding Remarks}

The Lake Huron Treaty process is a multidimensional historical geographical process that includes a variety of relationships at many levels. Cybercartography has provided a fruitful framework for the design and development of the Lake Huron Treaty Atlas, which begins to present the complex relational network that has developed over time and space, and that characterizes the ongoing Lake Huron Treaty process. The Lake Huron Treaty Atlas reflects the main components of cybercartography, and has also given rise to some new concepts and approaches. As a distinctively cybercartographic approach to atlas making, the Lake Huron Treaty Atlas acts as a bridging approach: incorporating both Anishinaabe and critical cartographic perspectives and approaches into the iterative design and development of the Atlas. 


\section{Tracing the Iterative Dimensions of the Lake Huron Treaty Atlas Process}

"Cybercartographic atlases do not appear in full bloom at a single instant in time, but rather tend to develop over time through an iterative process comprised of design, implementation, and testing phases. Each cycle in this process may involve the production of prototypes (paper and/or digital) around which discussions of both an atlas's conceptual content and cartographic possibilities can occur. A new iteration begins, based on these discussions, through the integration of atlas design and development decisions, feedback from testing, and additional stories and questions that may arise through discussion of information synthesized by already existing versions of the atlas. Story precedes atlas design insofar as it creates a context and purpose for communication but atlas narrative and representational possibilities develop in tandem" (Brauen et al., 2011, 29).

This chapter describes the general nature of the iterative processes involved in the conception, creation, review and revision phases of the geonarratives in the Lake Huron Treaty Atlas. Many of the iterative processes that have come into play in the making of the Atlas are features of the broader cybercartographic framework and are shared characteristics among all cybercartographic atlas projects. Performativity is central to iterative processes, which involve active collaborations and include diversity of Atlas contributors (Pulsifer et al., 2008; Brauen et al., 2011). David Turnbull's (2000) performative understanding of collaborative construction processes is useful for understanding the performative nature of cybercartographic atlases, the Lake Huron Treaty Atlas, in particular.

This chapter begins with an introduction to David Turnbull's view of collaborative construction as a combination of "talk, templates and tradition", full of movement, change and emergent knowledge; rather than a formalized, rationalistic and static account of collaborative creation processes. A summary of 
the iterative processes involved in the making of the Lake Huron Treaty Atlas is provided next, followed by a discussion of the multiple dimensions along which these iterative processes occur.

Collaborative relationships are essential to Atlas development. Atlas contributors come from a variety of diverse yet inter-related communities of practice (Pyne and Taylor, 2012; Pulsifer et. al, 2011), with some distinctive contributor roles emerging through the atlas-making process. The diverse range of map themes and content reflect the fact that the Lake Huron Treaty is a complex multifaceted process that involves a variety of interrelated scales, levels or spheres. The way geonarrative responds to this is the subject of the next section. This chapter ends with a discussion of the role of Anishinaabe perspectives in the iterative development of the Atlas, and draws attention to the ways they have been combined with critical academic perspectives.

\subsection{The "Stuff" of Iterative Processes: Talk, Templates and Tradition}

In chapter two of Masons, Tricksters and Cartographers (2000), David Turnbull goes against the common grain and develops a performative perspective of the construction process of the "Gothic" Chartres cathedral. Turnbull lists the types of questions that are commonly asked by scholars:

How were they designed? What was the role of the architect, of plans, drawings and of scientific knowledge? How were innovations like flying buttresses possible in the absence of a theory of structural mechanics? How were large numbers of undifferentiated stones assembled into an organized structure? How was the labour and skill of large numbers of men and women coordinated? (53)

Turnbull's performative interpretation of the construction process identifies and frees itself from the "presuppositions about the nature of the design process and about the nature of scientific and technical knowledge that 
have guided many other interpretations" $(2000,54)$. These other interpretations "answer these questions in a way that on the one hand makes the construction process seem mysterious and radically different from 'modern' construction and design, and on the other obscures the historical emergence of such technical devices as plans" (54).

According to Turnbull, the presuppositions inherent in conventional analyses dichotomize technology and science, the medieval and the modern the ad hoc and the theoretical. In doing this, they "create a divide between then and now", perpetuating the linear approach to development as progress that privileges the modern and casts pre-modern "man" as primitive and in need of divine intervention to accomplish their great feats of "Gothic" construction: “They portray science as an abstract and entirely modern phenomenon and cathedral building as mere technical craft unguided by theoretical or scientific understanding. Hence they are drawn to make an inexplicable mystery out of the Gothic cathedrals" (54).

Turnbull's performative approach to understanding the way the Gothic cathedrals were constructed involves viewing the Gothic cathedrals "literally as 'laboratories'" (54), in which iterative interactions occur between the three dimensions of talk, templates and tradition:

The power of laboratories derives from their being sites at which people, practices and the diverse but amorphous materials can be shaped, manipulated, assembled, and transmitted beyond the laboratory. There are three essential components in the transmission of the mix of knowledge involved in the construction process: talk, tradition and templates. Talk and tradition were vital to the interaction between the mason and the clerics and between groups and generations of masons ... (54). 
As different people from a variety of knowledge traditions talked with one another about various issues related to the construction process, transformations in knowledge occurred. One important manifestation of these transformations was templates: Guides that other people could use to continue patterns that worked. The people incorporated the templates into their practices in a way that in turn contributed to the emergence of new traditions, and the performative cycle of collaborative construction continued over several generations until the cathedral was completed (Turnbull, 2000).

This view is contrasted with a view of design and development as a hierarchical process, governed from above by the all-seeing eye of rationality and techno-scientific logic. It flies in the face of any one-size-fits all approach. It is a view that acknowledges the synchronicity and rhizomatic growth of both social relations and design and development knowledge (Hess, 2004; Caquard et al., 2009).

\subsection{Iterative Processes in the Design and Development of the Lake Huron Treaty Atlas}

Talk is a key ingredient of the iterative processes comprising the Lake Huron Treaty Atlas. In contrast with the construction process in the making of the Chartes, "talk" in the collaborative creation of the Atlas extends to text, and is often mediated by such technologies as the telephone and the Internet. The category of "templates" is a broad category, including but not limited to concept and design drawings, geonarrative concepts, and Anishinaabe stories. "Tradition" is a likewise broad category that includes this atlas project being a part of the reconciliation context referred to in chapter one, as well as being part of the cybercartographic tradition. 
The iterative processes involved in the creation of cybercartographic atlas geonarratives are linked to a non-linear, cyclical view of development (Cowen and Shenton, 1996; Nederveen Pieterse, 2009): incorporating past elements, bringing new elements forward, and leaving certain elements behind, with the possibility of re-integrating them into the development process after any number of project iterations. An atlas geonarrative has four phases of development: conception, creation, review and revision, with the review and revision phases recurring indefinitely throughout the life of the map in a cyclical and reflexive manner.

The iterative development of the Atlas has occurred both as a function of relationships occurring within the evolving atlas project, and as a function of relationships between the Lake Huron Treaty Atlas and other atlas projects hosted by the Geomatics and Cartographic Research Centre. Inter-project iterative processes involve building on, borrowing from or contributing to other atlas projects. This Atlas could not be what it has become without the other atlas projects. At the same time, it is distinct from them and has generated some unique design challenges and solutions, which can in turn be applied to other cybercartographic atlas projects.

There are also intra-project iterative processes, which occur within the life of a cybercartographic atlas project over its successive funding phases. The design and development of the Survey Journeys maps, including the emergence and evolution of "geo-transcription", provides a good example of this. The Survey Journeys maps are the only maps to exist across all three funding phases of the atlas project. Although the Critical Commentaries Map began in Phase I, it 
was not until Phase II that an actual map prototype based on this initial work was produced; and, this map has been carried forward into Phase III.

In Brauen et al. (2011), we described iterative atlas development in the following manner:

In our experience, digital atlas projects proceed iteratively as fora for the development and exploration of ideas related to a set of topics rather than simply as answers or manifestations of collected knowledge on those topics. This is especially true since these atlases are focused on finding ways to express multiple perspectives, each of which takes time to discover, explore, test, and integrate into an atlas. Iterative processes are manifested both within and between atlas projects in the sets of relationships that occur, in information transmission and exchange, in design and development decisionmaking, and implementation more generally (28).

Interactions, learning, emergent knowledge and transformation are central aspects of the iterative processes that give rise to the Atlas with its maps. These iterative processes occur along four interrelated dimensions: conceptual; financial; technological; and content. For example, in the case of the technological dimension, "common to the [Lake Huron Treaty Atlas] project and other [Geomatics and Cartographic Research Centre] research projects is the software base upon which these atlases have been created. This base is developed iteratively in conjunction with this and other atlas developments and is released as "free and open source software (FOSS)" (Brauen et al., 2011, 29; Taylor and Pyne, 2010).

\subsection{Multiple Dimensions: Inter-dimensional Iterative Processes}

The Atlas has developed since its inception in an iterative manner along the interrelated conceptual, financial, technological and content dimensions (Brauen et al., 2011; Pyne and Taylor, 2012; Pyne, 2013). Each of these categories is construed broadly. For example, the conceptual dimension includes 
epistemological and ethical dimensions, consistent with a holistic approach to rationality (Pyne, 2006), in addition to ontological and methodological considerations. Evidence of the role of the conceptual dimension is apparent in the design decisions that have contributed to the development of the Atlas. Conceptual development is also expressed significantly through the various writing and presentation outputs associated with the atlas project. These include peer-reviewed papers (Pyne and Taylor, 2012; Brauen et al., 2011; Taylor and Pyne, 2010; Caquard et al., 2009) book chapters (Pyne, 2013; Pyne, 2012) funding proposals (Inukshuk Grant, SSHRC Standard Research Grant and SSRCH Outreach Grant); academic papers, including the comprehensive paper and the thesis proposal associated with this project (Pyne, 2008, 2009); and, numerous presentations both to academic and non-academic audiences (see Appendix B for a list of presentations).

The "financial" dimension refers primarily to the various grants that have underwritten the atlas project. Grants have interrelated aims, guidelines and an overarching ethics, which the funding applicants must essentially "comply with" in order to even qualify for being considered in a funding competition. These aspects shape and guide successful grant applicants in carrying out their research projects.

The "technological" dimension is manifest primarily in computer programming and geospatial technologies development activities; it is heavily contingent upon the funding dimension. In the case of the Lake Huron Treaty Atlas and all other cybercartographic atlas projects, the technology that underwrites cybercartographic atlases is free and open-source software (FOSS), a sharing community of software developers with a common ethic. As we summarize in 
“Encouraging transdisciplinary participation using an open source cybercartographic toolkit: The Atlas of the Lake Huron Treaty Relationship Process":

Politically, the decision to use and create FOSS relates to the material effects we anticipate for mapping projects. The constitutive effects of mapping and the discussions and understandings that can arise through outreach from a mapping project are ... the main impetus for our projects. Enabling a diversity of groups to participate in atlas projects is a desirable outcome of our research, related also to the potential for multiple perspectives to be accommodated within the narrative of a single atlas (Taylor and Pyne, 2010), and is best achieved if the cost barriers to adoption and use of the technologies we produce are kept as low as possible. In this way, our participation as a FOSS producer and user inserts our project into what Christopher Kelty $(2008,3)$ has called a recursive public: "a public that is vitally concerned with the material and practical maintenance and modification of the technical, legal, practical, and conceptual means of its own existence as a public." Kelty argued that participation in the creation and use of FOSS is one way in which such a public asserts itself as "capable of speaking to existing forms of power through the production of actually existing alternatives" (ibid.). We would argue that enabling the creation of spatial narratives through the production of open source technologies is consistent with the vision for such a public.

By choosing to develop a team around FOSS, we chose, possibly without knowing it, to develop a core set of capabilities related to web mapping, geospatial technologies, and open geospatial standards within our project that would not have developed if we'd had a vendor-provided set of tools (Brauen et al., 2011, 32).

The "recursive public" focus of the FOSS movement combined with its interest in non-proprietary information and creating an equitable community for software knowledge sharing, makes it a good choice for atlas design and development along the technology dimension. Finally, the "content" dimension relates broadly to the multimedia "stories" identified for transformation into geonarratives. In Brauen et al. (2011), we describe the general nature of different kinds of content:

Content contributions to this cartographic telling of the Robinson Huron Treaty story come from a variety of interrelated, and 
sometimes overlapping knowledge perspectives, including Anishinaabe, historical, archival, survey, archaeological, legal, geographical, political, and critical academic understandings. In keeping with the multimedia orientation of the project, contributions can be text, images, videos, or audio (33).

The iterative development of geonarratives involves interactions between these four dimensions in a holistic interplay of theory and practice. While it is surely difficult to understand the inter-relationships between these four dimensions in the abstract, it is useful to have a general understanding of the geonarrative design and development terrain when discussing specific aspects of the making of the Atlas, to follow in this thesis.

\subsection{Collaborative Relationships}

Collaborative relationships are central aspects of the types of iterative processes that give rise to the Atlas. The contributors and their roles in the collaborative creation of the Lake Huron Treaty Atlas have ebbed and flowed over the years since the project's inception. Throughout the three phases of this atlas project, new contributors have emerged and new roles with them (see Appendix C for a general summary of fieldwork during phases II and III, including examples of collaborative content gathering and atlas design and development activities).

In Brauen et al. (2011), we draw attention to the need for mutual understanding in the transdisciplinary approach to cybercartographic atlas making when it comes to dealing with the presentation of "complex" subject matter:

Concerning the subject matter of a digital atlas, considerations contributing to complexity include selection of data appropriate to the study of a problem; how or whether these data relate to each other at different scales; and which scales are appropriate to the topic. Within the dynamics of transdisciplinary research, intricacies 
inherent in communication between collaborators from different knowledge communities (Lauriault and Taylor 2005) may compound this complexity. However, an emphasis on continual learning across knowledge communities may help to reduce complexity in some cases, a fact that is acknowledged in transdisciplinary research (Lawrence and Despreés, 2004; Ramadier, 2004; Lauriault and Taylor 2005; Mobjörk, 2010). In a sharing and learning context, the development of ideas and collaboration among members of a transdisciplinary team and participating community members as process outputs are as important as the creation of an atlas artefact. This approach to digital atlas making aims at increasing mutual understanding between project collaborators, a goal that is being increasingly emphasized in the product design literature (Hughes and Chafin 1996; Pahl and Grote 1996; Winters and Mor 2008; Durdu et al. 2009). By applying this transdisciplinary research ethic in the process of crafting a story into an interactive map, a subject matter 'expert' may come to learn more about cartography and geomatics; while cartographers and geomatics specialists may learn and appreciate story details, in turn enhancing their ability to recommend geospatial representational solutions to problems and challenges associated with expressing and communicating the story (28).

Another way to view complexity is in terms of multiple rationalities or knowledge systems (Turnbull, 2000). Mutual understanding across disciplinary and other knowledge space barriers is a key component of successful collaborations. An interesting observation in this regard concerns the relationship between the process of coming to know and the knowledge gained via this process. It is imperative that the process reflects the same values as the values inherent in the knowledge being sought. In this case, the Lake Huron Treaty Atlas aims at contributing to mutual intercultural understanding along many dimensions, so it is appropriate that it is able to achieve mutual understanding in the process of becoming a tool that promotes it.

Cybercartography involves a team approach that goes beyond the individuals formally involved in specific atlas projects, and that contributes to inter-project iterative processes. For example, it is common for all GCRC 
members to be included in group-emails concerning the Centre and its various projects. More specific to the atlas project is the example of the way work to develop an atlas portal to the maps has benefitted from ongoing work to improve the concept map in the cybercartographic Inuit Siku (Sea Ice) Atlas.

Although atlas-making roles and responsibilities have shifted and changed throughout its three phases of development, some identifiable trends have emerged through the process. For example, in the process of preparing this thesis, I have come to refer to myself as the geonarrative and content editor. The shift to adopting this title has happened in an iterative fashion throughout the development of the atlas project. Summarizing the beginning of the project in Caquard et al. (2009), I described my role as the "Treaties Module designer" who "became positioned both as a mediator and as an equal participant in the process of creating a cybercartographic geo-narrative (or historical geographical story) of the Lake Huron Treaty negotiation, signing, and survey processes"' (87).

In Brauen et al. (2011), the subject matter 'expert' aspect of my role was emphasized: "subject matter expertise is required to collect or generate data to be used in the atlas, which includes collaborating with groups or communities and generating ideas for how to represent these data meaningfully within the atlas"(28). In the article, we placed 'expert' in single quotations to qualify the use of the word. In reality, my role involved being a jack-of-all-trades, with emerging knowledge in many areas - including historical, archival, survey-related, and cartographic - that has allowed me to communicate with a diverse range of Atlas collaborators from a diverse range of knowledge spaces.

We also refer to the "storytelling" and "geonarrative mediator" functions of my role: 
Throughout both phases of this project, the atlas-making process has involved a storyteller (or storytellers) working in collaboration with team members and other atlas-making contributors to render a series of interlinked stories into a mapped geonarrative form. Whether in the domain of content or implementation, the overall required skill set necessary to produce these geonarratives is often comprised of a diverse range of skills and knowledge. In addition to the skills required to write and develop the story itself and create media in support of it, the creation of a geonarrative requires team members possessing an overall set of combined skills including data collection and processing, cartographic and geographic information processing, and computer programming and configuration. Throughout this process, the storyteller functions as a geonarrative mediator, conducting research and coordinating the information and perspectives coming from different content-related sources, maintaining communications where necessary between a variety of content-contributing collaborators, synthesizing contributions into ideas for atlas-related geonarratives, and working with geospatial technologies specialists to implement these ideas (33).

Reflecting the difficulty of summarizing my role with a single descriptor, I chose in Pyne and Taylor (2012) to include my role under the general description of "those responsible for the geonarrative content", a broad category to contrast with "the software-design and information-infrastructure team" (96): "Although they fall into two relatively distinct knowledge domains, these two groups share intersecting knowledge and responsibilities when it comes to atlas design and development, and the expansion of knowledge that results from their interactions contributes to the emergence of maps" (Pyne and Taylor, 2012, 96).

In this thesis, I refer to my role as geonarrative and content editor, following James Akerman's (1991) characterization of the atlas editor.

Dr. Fraser Taylor has been the atlas project's principal investigator across all three funding phases, in addition to being my doctoral thesis supervisor. In the case of the atlas project, where the publication of an atlas version has been an outcome in both phases I and II, the principal investigator role is akin to 
Akerman's “Atlas publisher" (1991). Dr. Taylor's ongoing knowledge of cybercartographic atlas design and development processes has been indispensible to the effectiveness of his advisory and supervisory role.

Sebastien Caquard acted as a coordinator and cartographic facilitator during phase I of the project, which gave rise to the cybercartographic Atlas of Indigenous Perspectives and Knowledge, including the Treaties Module. In phase III of the project Sebastien's role has shifted to an advisory role with respect to MA student, Tom McGurk, who is working as a research assistant with artist Nadia Myre on a collaborative project to map the Scar Project.

Glenn Brauen has been instrumental in the role of geospatial and cybercartographic technologies specialist throughout both phases II and III. Glenn's ability to mediate between narrative and technological dimensions through his ongoing knowledge of both the stories being told in the Atlas and the stories behind those stories, in addition to his keen cartographic and programming skills have led him to create of some innovative software design solutions to the geonarrative challenges he has been faced with. Some of these solutions will be discussed further in chapter 8 .

Amos Hayes, the Geomatics and Cartographic Research Centre's Technology and Laboratory Manager, participated in the development of both Nunaliit I and II. Amos has an ongoing knowledge of the technology underpinning the Atlas and of technology resource logistics. During Phase I, Amos was more involved in the geonarrative aspect of atlas development, while in phases II and III, his role has been focused more on atlas software framework development. 
More details will be provided in the next chapter of the various roles, responsibilities and contributions made by increasingly diverse range of Atlas collaborators, including Geomatics and Cartographic Research Centre team members. Common to all collaborations is the idea of community, the idea of working together. Bringing together a diverse range of skills and knowledge in the creation of a geonarrative space, the atlas project involves taking the time to let iterative processes play out through the making and sustaining of collaborative relationships.

\subsection{Iterative Processes and Geonarrative}

The diverse range of map themes and content reflect the fact that the Lake Huron Treaty is a complex multifaceted process occurring along a variety of interrelated scales. Recognizing the difficulties inherent in separating these, this project has involved critical and alternative mapping activities in three broadly defined and overlapping spheres: Governance/legal institutions, political economic and interpersonal-lived experience. The emergence of "geotranscription" as a specific approach to geonarrative method, to be described in

chapter 8 , provides a good example of how the four dimensions referred to above - conceptual, financial, technical and content - came into play. It is important to note here how conceptual development relates to methodological development. In this thesis project, the emphasis has been on the importance of finding the way to approach the design and development process. This way has involved incorporating both Anishinaabe and critical academic perspectives and placing performative emphasis on emergent Atlas development through iterative processes. 


\subsection{Iterative Processes and the Anishinaabe Perspective - Concluding Remarks}

The shape, form and direction of the iterative development of the Atlas have been influenced by the Anishinaabe perspective in a variety of ways, on a variety of occasions, and in a variety of contexts. This has been an aim of the atlas project from the beginning: To acknowledge and incorporate Anishinaabe perspectives both in the content of the Atlas and in the approach to its making. This original mandate has been carried forward through successive phases of the atlas project.

Soon after the commencement of the project, it became apparent that it was not only vital to include the Anishinaabe perspective in order to acknowledge and respect it, and to participate in the regeneration and transmission of culture; it was also vital to the enrichment of the project itself. Incorporating the Anishinaabe ways that went beyond narrowly defined content contributions has led to the emergence of some very interesting and useful knowledge, and broadens the perspective of history and geography. In conjunction with many others, I have had the unique privilege as the geonarrative and content editor to apply my Anishinaabe-influenced approach. Guided by this influence, I have had the opportunity to make some key editorial design and development decisions, subject to the approval of the project's principal investigator and publisher (and my thesis supervisor), Fraser Taylor; and guided by knowledge from team members and atlas contributors coming from various communities of practice or knowledge traditions: from geospatial technologies, to historical and archival, to artistic and traditional knowledge communities and beyond. Inviting people to participate in a collaborative project 
to share perspectives and skills with the common broad aim - to contribute to the enhancement of mutual understanding in a way that responds to the reconciliation challenges of today.

In the case of the Lake Huron Treaty, there are many stories that have not been heard. People are only now beginning to share. Until recently, and still lingering today, there have been many barriers to mutual understanding. The age of inter-, multi- and trans-disciplinary research has contributed much to making these barriers permeable. Some of the chinks in this armor have been made by the cross-disciplinary spatial turn to performance (discussed in chapter 4) and the interpretive turn to reflexive narrative (discussed in chapter 3).

A key principle of all cybercartographic atlas projects, including the Lake Huron Treaty Atlas, is that the processes by which knowledge is attained are part of and central to the knowledge itself. These processes ultimately involve relationships between people from intersecting knowledge traditions, sharing through talk and contributing to the development of emerging templates for creating a new awareness in the form of geonarratives. The next chapter will describe some of these interactions in more detail. 


\section{Part IV}

\section{Waasaa-inaabidaa: We Look In All Directions}

I chose this heading for Part IV to emphasize the non-linear approach taken in this project. The narratives presented in this part are reflexively intertwined with the atlas maps they reflect on insofar as they draw on the narrative logic of the maps, in addition to interpreting those maps and providing new material and ideas to further populate these same maps. Presenting discussions concerning map and atlas development from two different, yet complementary, perspectives (the Travels in the Making of the Atlas Map perspective in chapter 8 and the Map of Maps perspective in chapter 9) gives rise to different yet related accounts.

Although chapter 8 is limited primarily to points in the Travels in the Making of the Atlas Map ending with the commencement of phase II, in cases where I am describing collaborations with individuals that continued past phase I, the discussion is extended to acknowledge this. This "story circle" approach (referred to in chapter 8 ) is one way of breaking away from a linear chronological approach to narrative.

The Map of Maps itself subverts conventional western approaches to space and time through its placement of points for the other maps in the atlas. Chapter 9 draws attention to this through its comments on ways the Map of Maps highlights the narrative logic inherent in the relationship between the maps along thematic, temporal dimensions. 


\section{Reflexive Tracking of the Treaties Module in the Travels in the Making of the Atlas Map}

This chapter provides primarily a chronological telling of some significant events in the making of the prototype Treaties Module in the Travels of the Making of the Atlas Map. It is intended to convey the emergent nature of the iterative processes I was involved in as the geonarrative and content editor. Although, at times I settle into a story and skip the chronological queue - so to speak - telling that story in its own circle. As Bordieu said in his introduction to a lecture on incorporating reflexivity into social science research, it is often necessary to give a "high-speed " tour:

One of the functions of a seminar such as this one is to give you an opportunity to see how research work is actually carried out. You will not get a complete recording of all the mishaps and misfirings, of all the repetitions that proved necessary to produce the final transcript, which annuls them. But the high-speed picture that will be shown to you should allow you to acquire an idea of what goes on in the privacy of the "laboratory" or, to speak more modestly, the workshop - in the sense of the workshop of the artisan or of the Quattrocento painter-i.e., it will include all the false starts, the wavering, the impasses, the renunciations, and so on. Researchers whose work is at various stages of advancement will present the objects they have tried to construct and will submit themselves to the questioning of all the others who, in the manner of old compagnons, fellow-workers of the trade, as they say in the traditional language of the métiers, will contribute the collective experience they have accumulated over all the trials and errors of the past" (Bordieu, 1992, 220).22

The Travels of the Making of the Atlas Map provides an initial sketch of some of the stories behind the ongoing Lake Huron Treaty Atlas in its earlier days. This map presents traces of my journey through Anishinaabe country and

\footnotetext{
22 While Bordieu's description is appropriate in terms of its emphasis on the usefulness of a "high speed tour", in this project there are no "mishaps and misfirings" that annul the emerging atlas. In contrast, all of the interactions, and attempts to understand and create, ultimately comprise the atlas as a performative product.
} 
beyond. It tells of the people I met, the ideas I encountered, and the ways these mapping encounters contributed to the iterative development of the Atlas. Most of the stories in this narrative reflection can be folded back into the Travels in the Making of the Atlas Map, illustrating the reflexive nature of the relationship between this thesis and the Atlas it comments on. Further 'populating' the Travels in the Making of the Atlas Map with the interpretive thoughts from the thesis is one way of sharing them with the broader community, extending beyond the academic community. It is also important to stress the "in-themaking" aspect of the Travels in the Making of the Atlas map and the other maps in the Atlas. For example, currently more than halfway through phase III of the project, an entire year of journey stories remain to be added to extend the Travels in the Making of the Atlas Map.23

In addition to the various papers, chapters and presentations revolving around the atlas project over the years, I consider all of the activities, including meetings, conversations, email and telephone exchanges and ideas transmitted through online social networks to be a part of the Atlas, which is a performance in itself. In this reflexive tracking narrative I try to give people the picture of the iterative nature of the Lake Huron Treaty Atlas and an idea of the significance of incorporating Anishinaabe perspectives into the Atlas-making process.

This reflective telling will reveal some of the details concerning the iterative processes contributing to the emergence and early development of the Lake Huron Treaty Atlas, in addition to highlighting the reflexive and performative aspects of these processes. The Atlas' holistic features are demonstrated through

23 Work to add the outstanding media from previous years is awaiting an upcoming conversion of the Atlas to a new geospatial software framework, which will afford improved functionalities in a variety of areas. 
the iterative processes that were involved in the first phase of the atlas project, the Treaties Module of the Atlas of Indigenous Perspectives and Knowledge.

The Travels in the Making of the Atlas map is my personal counter-map to the Survey Journeys maps based on J.S. Dennis' official survey diary. It reflects my attempts to put into practice and thought the critical cartographic call to "rethink" and re-do" mapping. The reflective tracking in this chapter ends with the official beginning of phase II in order to provide some details on the inter-phase transition between phase I and phase II. A full account of the entire Travels in the Making Map would be too unwieldy.

\subsection{The Story of How I Became the Treaties Module Research Assistant}

The Travels in the Making of the Atlas Map begins in May 2007 in Ottawa where I was living. I had just finished my first two terms of the PhD program, and my required journey into the coursework portion of the program, with the completion of the last of my required coursework papers, "The political economy of relational space" (Pyne, 2007). I had spent the past eight months prior to this learning about the geographical perspective, and focusing on an emerging concept in critical geography, "relational space". This approach to space was very helpful to my overall project to identify and transform deep Western European assumptions; it linked well with my previous work in the Master's of Philosophy program to present a holistic view of the relationship between "reason" and "emotion" and to point the implications of this understanding for development thinking, reconciliation and inter-cultural bridge building. It is interesting that before I had learned about the opportunity for the research assistant position with the Geomatics And Cartographic Research Centre, I was already formulating an initial doctoral thesis focus with a mapping theme, which I 
expressed in "The political economy of relational space" as a project "concerned with mapping the moral politics of emotionally mediated interpersonal dynamics in the ongoing transformations of formal and non-formal institutional spaces" (Pyne, 2007, 2).

During the first two terms of the PhD program, I had done some conference reporting and writing for the Research Resource Division for Refugees (RRDR, now called the Centre for International Migration and Settlement Studies). Now that the pressure of paper deadlines was over, I could turn my attention to how I was going to make a living over the summer until my teaching assistant duties (and pay) were resumed in the fall. At that time, I had one potential assignment with RRDR reporting on a conference in Edmonton, which I would also attend. However, this assignment was pending a contractual agreement between the University and RRDR, which was still not finalized the day before I would have to fly to Edmonton. Finally, near the end of the workday on around 3:45 p.m. on May 22, 2007, my son's cellphone rang while we were driving. It was my mother telling him that I should call the RRDR administrative assistant right away. The agreement had been reached, the funding could be released and I could go to cover the conference in Edmonton if I called back before four. Normally, I would make the call right there and then, but this time something stopped me, and I told my son that I would be home before four and would call then.

I arrived home several minutes later and phoned the RRDR; but the administrative assistant had left for the day and I had missed the opportunity to cover the conference. Now, more intent that ever to find a summer source of bread and butter, I went to my computer and checked my email. To my eye- 
opening surprise, the first email I opened was the call for research assistants to work on the cybercartographic Atlas of Indigenous Perspectives and Knowledge, which was envisioned to contain three modules. I chose to outline why and how I would be "perfect for the job" of gathering and cartographically organizing some relevant content to create the Treaties and Territorial Claims module of the Atlas (The Treaties Module). Less than 24 hours after submitting my application, I had the position!

Early on, after learning just a little about Fraser Taylor's cybercartographic atlas-making framework, I began to see a great potential for multidimensional storytelling ... my imagination was engaged and my subsistence issued erased. Timing in decision making: If I had returned the call from RRDR right away, I would have gone home to pack for the trip to Edmonton and would have been too preoccupied to check my email for other possibilities. There is a lesson in this story about learning how to 'go with the flow'. It is not necessarily an easy discipline, but it works! And it worked for me in a way answered by subsistence concerns and led me to my thesis vision, which would emerge through the process of beginning to fulfill my duties as the research assistant responsible for developing content for the Treaties Module.

I consider learning to "go with the flow" to be a Anishinaabe discipline. Synchronicity is an indication that a person is on the right path that links their skills, talents and gifts with their purpose. The way to do it is to follow your heart, which involves learning to how to "know" your heart, and implies a holistic relationship between heart and mind: Connecting past, present and future in a synergistic manner that involves meaning, and that goes beyond causal and (merely) correlational approaches to temporal relations. 
In my travels, I have heard many Elders talk about this, and I have tried to develop my ability to 'go with the flow' in my editing approach to the Atlas. This is an important aspect of the Anishinaabe approach that has been incorporated into my way of doing what is commonly referred to as "cartographic research". I had the choice in that instant - a short period of time - to phone the RRDR administrative assistant right away after getting the message. I could have pulled off the road and returned the call then and there; and, within minutes I would have changed my life path, and would not be writing this thesis today, but a different thesis - who knows what? And so, I began participating in the iterative processes involved in the conception of the Survey Journeys Maps.

\subsection{Getting my Feet Wet - Swimming in the Seas of Cybercartography ... and History}

The Treaties Module was not always focused on the Lake Huron Treaty region. At the beginning of the project, the context was a fairly broad definition of the Great Lakes region. Because this was a project to develop sample content, it was clear that after a certain period for preliminary background research, a decision would have to be made with respect to historical and geographical focus. In addition to my prior knowledge and understanding, I began with learning about "cybercartography" as a theoretical and practical discipline to get a better idea of the conceptual and design framework (Taylor 1997, 2003, 2005, 2008, 2013).

"Is there a guidebook or a manual for making cybercartographic atlases?" I asked Amos Hayes, the laboratory technical manager. Amos replied that it was best to come into the lab to work and to learn by osmosis, since most of the knowledge about the atlas making could be learned in the process, through 
experience. The experience of my early days working on the Treaties Module reminded me of my first days working as a legislative revisor for Justice Canada: Reading over background information and beginning immediately to participate in some of the "shop" activities. In both cases, as I began participating more, the ideas expressed in the background material I had been reading began to come to life and have new meaning for me.

My first meeting with Fraser Taylor, within a week of beginning my new RA position, was memorable. We discussed the new and exciting possibilities for presenting multiple perspectives in multiple ways that were emerging in cybercartography and the links with my early thesis ideas to map multiple dimensions of relational space. We also discussed the "treaties" focus of the content module that I was responsible for developing as a research assistant, and how that would tie in with my doctoral studies. I recalled that through my work editing legislation and reporting the House of Commons Debates, I had once wanted to devote a $\mathrm{PhD}$ to studying alternative forms of "jurisprudence" that would better reflect Indigenous perspectives and understandings. It surely seemed that this research assistant position was tying many things together. Fraser was looking for students to use his framework to create atlas products for understanding sociopolitical and economic issues, and I was looking for a way to map relational political economic space. Soon after, I would formally request that Fraser be my thesis supervisor.

After the meeting, I went back to acclimatizing myself to the cybercartographic atlas-making environment, and tried out some mapping ideas suggested in the project description: 
The original mandate [...] was to demonstrate the efficacy of cybercartography in representing information relating to territorial claims and in encouraging Indigenous and other community participation. This would involve collecting data and content related to territorial claims in the Great Lakes-St. Lawrence region and transforming it into geonarrative form (with the aid of other GCRC team members) in a manner that would be meaningful to a broad audience, with the added requirement that the content should reflect Indigenous perspectives. In addition to a variety of historical and contemporary research, a polygon-drawing exercise was initiated to gauge the feasibility of mapping out the written descriptions of a selection of historical treaty and surrender documents (Brauen et al., 2011, 33).

It turned out that the polygon-drawing exercise brought up many questions. For example, sometimes the written descriptions provided in the Indian Treaties and Surrenders (Chamberlain, 1891) were not detailed enough. Other times changes in landscape made cartographic interpretation difficult. In addition to this, I did not have a formal Geomatics training; this made me a good "guinea pig" for further development of the framework, which aimed to be user friendly.

I went to visit Steve Prashker, a Geomatics specialist at Carleton University, who happened to be working on a polygon drawing exercise for a land claims research project. He showed me what he was working on and explained the challenges that he - as a trained professional - was experiencing with the exercise. In addition to the extended time it takes to complete a polygon of a written treaty or surrender description, a considerable amount of expertise was required in order to complete the task. After this, I continued doing historical research into Ontario treaty history, keeping my eye open for interesting narrative leads. 


\subsection{Land in Sight - Lake Huron Treaty Region Aho(y)}

The first significant Anishinaabe contribution to the map-making process happened about five weeks into the research project in July 2007 when I received a forwarded email from Alan Corbiere, then-Director of the Ojibwe Cultural Foundation, through Dr. Heidi Bohaker, a University of Toronto historian and Atlas-making team member. Alan had learned about the atlas project through Heidi and Dr. Ruth Phillips, a co-investigator on the project.

Alan's email referred to a paper by Michael Marlatt, “The Calamity of the Initial Reserve Surveys under the Robinson Treaty" (2004), which includes a relatively detailed description of the extended survey process for the Lake Huron and Lake Superior region treaties of 1850. Alan also included a particular mapping suggestion, which is still on the agenda.

After reading the paper, I was sure that the story of the Lake Huron Treaty signing and survey process would serve as ideal sample content for the Treaties Module: a perfect example for a cybercartographic geonarrative designed to include previously excluded worldviews. To me, Marlatt's chapter was ideal since it provided a detailed account of the incompleteness, inconsistencies and incorrectness of the survey process, and revealed the type of story I thought needed to be told: A story that reflected the many layers in the treaty relationship, and that was particularly critical of the colonial aspects of treatyrelated processes.

Up to this point, in addition to the knowledge and experience that I brought to the project, I had an emerging knowledge of (1) the parameters of the project; (2) cybercartography as a theoretical and methodological framework and the first two Atlases produced by the Centre; and, (3) treaty-related history - yet 
still, I was still not sure exactly 'what' to map, or how. After this point, I was sure. Amazed at how much more there was to the Robinson Huron Treaty than the actual treaty document and impressed by the research in the Marlatt chapter, I now had a working vision for a map and a story to focus on. The experience associated with this significant decision point, which resulted in the initial map conception, can be referred to as a "mapping epiphany" (to be discussed in chapter 10).

\subsection{Post-epiphany Focus}

After this initial mapping epiphany, which resulted from the mapping encounter via email, things really got moving. I began to pay attention to Robinson Huron Treaty-related history, in particular, and studied the Marlatt chapter, this time from a mapping perspective that generated ideas concerning how to present the Marlatt chapter in geonarrative form. With the idea of context looming largely in my mind, I was looking for ways to map the Marlatt chapter that included additional stories about the places that would be referred to in the map summarizing his chapter. During this time, I created a PowerPoint prototype, which laid out some of the emerging map's contents based on the Marlatt chapter, with plans to construct a map based on this by February 2008.

I continued working on the Marlatt map, which included some additional reading about treaty-related matters. Another important aspect of this work included attending weekly meetings with the atlas-making team. In one of our early meetings, Heidi Bohaker provided some Anishinaabe historical background and discussed issues related to doing research in a decolonizing way and Onondaga artist Jeff Thomas discussed his work. 


\subsection{Story Circle - Patricia Kennedy and the Governance Paper Trail Map}

Following an initial introduction email from Heidi Bohaker to Patricia Kennedy, a recently retired senior archivist at Library and Archives Canada, Patricia and I met for the first time on August 16, 2007. Over the years since then, Patricia and I have managed to meet according to her personal schedule about four times a year for discussions lasting between four and seven hours. Fairly early in our meetings, we began talking about ways to map some of the invaluable knowledge Patricia was sharing with me concerning archival history, archival research strategies and archival collections details. The Governance Paper Trail Map was born out of these discussions. Our discussions are audio recorded. A small selection of clips from these meetings is included in the map, and one day more of this audio will be incorporated. An initial experiment was attempted to build the map on a conventional cartographic background prior to the launch of the Atlas in May 2012; however, we have been thinking all along that a more abstract, conceptual background map would be more appropriate; and, we are awaiting the outcome of the work to improve the concept map of the Siku (Sea Ice) Atlas before moving ahead with further development of this map.

In addition to sharing a wealth of knowledge with me, from the specific Robinson Huron Treaty process-related information to broader, more contextual information such as the changes in British colonial administrations over time and the characters and relationships of some of the individuals who filled official administrative positions, Patricia has also made some useful comments regarding web presentation that reflect (1) her extensive experience and interest in presenting archival information to the public in an accessible fashion, and (2) her archival code of ethics regarding the need for people doing archival research to 
be aware patrons' archival research strategies. For example, one very relevant topic to be aware of in archival research is 'document authenticity'. Under Patricia's instruction, I learned about the many copies that were made of official documents such as the Robinson Huron Treaty, and that there were certain typical signs of authenticity to look for when examining archival documents.

\subsection{The 43rd Algonkian Conference, Toronto, Ontario, October 18-20, 2007}

At the 43rd Algonkian Conference, I met some of the key Robinson Huron Treaty and related issues historical researchers, people like Karl Hele, Rhonda Telford, Victor Lytwyn, David McNab, Darrel Manitowabi, Dan Shaule, Heidi Bohaker and Darlene Johnson. This conference was a great venue for me to meet people who were interested in and knowledgeable about treaty history, and who could relate to my ideas for mapping the Marlatt chapter. Rhonda Telford told me about how she had been the person to urge Michael Marlatt to write the chapter the map would be based on. Dan Shaule told me about his Master's work to shed light on the Williams Treaty, especially about the negative effect that the Constitutional division of powers had on effective treaty implementation.

Following the conference, I contacted Rhonda Telford and Karl Hele by email in search of Michael Marlatt's contact information. I wanted to get in touch with Michael and tell him about my plan to create a map based on his chapter. Rhonda got back to me with Marlatt's email address. Karl responded with an offer to send me a box of papers on Robinson Huron Treaty region history. ${ }^{24}$

\footnotetext{
24 One important paper, "Who is on Trial? Teme-augama Anishnabai Land Rights and George Ironside Junior: Re-considering Oral Tradition" (McNab, 1998), questions whether or not Temeaugama Anishnabai falls under the Robinson Huron Treaty or not. The Teme-augama Anishnabek maintain - despite Court order - that their ancestors were not party to the Treaty.
} 


\subsection{Reaching Out - Early Collaborator Contacts}

After the conference, I returned to Ottawa to work on the Treaties Module, and would stay there working until my first field trip on February 14, 2008. In addition to having our regular weekly atlas development lab meetings, I met with Patricia Kennedy in October and December. On November 21, 2007, I contacted Michael Marlatt by email. Michael's help would be indispensible in the making of the Survey Journeys maps. Wanting to share my plans to map the Marlatt chapter with some of the people from the communities referred to in the chapter, I began to contact band representatives in early November. In my mind, since the map would be dealing with issues concerning nation-to-nation relationships, it was important for me to speak with national representatives whose concerns were with treaty interpretation and implementation issues. I phoned a number of Robinson Huron Treaty region community band offices, and asked to speak with the Gimaah (roughly translated to Chief) or a band councillor with a knowledge of or interest in treaty related issues. Realizing that within the project timeframe, I would not be able to work with people from all of the 17 communities, I engaged with the late Blaine Belleau (then a Band Councillor) from Gitigaan-ziibing (Garden River First Nation), Gimaah Denise Restoule from Dokis First Nation, Gimaah Shining Turtle from Wigwaasminising (Whitefish River First Nation, Reserve No. 4) and Gimaah Art Petahtegoose from Atikameksheng Anishnawbeg. I would have several excellent conversations concerning treaty-related issues and the map project with each of these individuals over the following months, and in January would arrange to go to their communities for a visit. 


\subsection{An Unexpected Mapping Epiphany}

In January 2008, a surprise iterative transformation in map design and development occurred, hours after I participated with Alan Corbiere (mentioned above) and Amos Hayes, the Centre technical laboratory manager, in mapping the traditional story of Nenboozhoo and the creation of Mindemoya Island for the "Culture" section of the Atlas of Indigenous Perspectives and Knowledge:

According to this story, Nenboozhoo was in the Lake Erie region playing all kinds of tricks on people in all sorts of ways. Eventually, the people chased him out and he ran away, heading for Manitoulin Island, carrying his grandmother on his back. After travelling across the water from the Bruce Peninsula to the southern shore of Manitoulin Island, Nenboozhoo began to run toward Mindemoya Lake. Finally, he could no longer carry his grandmother; when he reached the lake, he threw her into it. She became what is today called Mindemoya Island: "Enh, mii 'mindemoya' enji-zhinkaadeg. Mii dash (Yes, that is why it is called Mindemoya" (Johnny Debassige speaking in Anishinaabemowin; English translation by Alan Corbiere). In the atlas, this journey has been rendered through a multimedia map showing the different steps of Nenboozhoo and his grandmother (see Figure 6). Audio files of Johnny Debassige telling the story in Anishinaabemowin have been added, along with English translations by Alan Corbiere. The combined Anishinaabemowin/ English translated text enables the story to remain in its original language while at the same time making it accessible to a wider audience (Caquard et al., 2009, 94).

Although I already had a map idea with the decision to render the Marlatt

chapter into a map, participating in the creation of the Nenboozhoo Mindimooye Interactive Story Map, which essentially tracks Nenboozhoo (aka Nanbush) the Trickster from east to west, gave rise to a second epiphany in the iterative map design and development process. John Borrows (2002) has written about Nanabush participating in contemporary legal proceedings, so it is no wonder the Trickster might want to participate in a decolonizing mapping project. In a similar fashion, in Debassige and Pyne (2012), Lewis Debassige draws attention to the importance of keeping the Nanabush spirit alive in ongoing efforts to 
establish a fair balance in Nation-to-Nation relationships between the Anishinaabe and the Crown. ${ }^{25}$

At this point, I had already had phone conversations with Michael Marlatt who had sent me digital copies of archival records including the Dennis survey report, diary and field notes (Dennis 1851, 1853), and sketch maps. I had also ordered a high-resolution digital copy of the Vidal-Anderson Commission 1849 Sketch Map (Vidal and Anderson, 1849) from Library and Archives Canada, which Alan had suggested I do. That evening the idea was born to transcribe and map out - or geo-transcribe - the Dennis survey diaries (Caquard et al., 2009):

The geonarrative focus shifted to a consideration of a more direct "tracking" of the surveyor. Although an initial map idea had already been generated and discussed, the experience of mapping out Nenboozhoo's journey to Mindemoye had inspired a new yet related map idea: To transcribe and map out J.S. Dennis survey diaries, the primary document upon which the Marlatt chapter was based, in addition to mapping out the Marlatt chapter as a critical geonarrative summary of the survey diaries. The diary referred to more than 100 distinct campsites, provided additional details to the Marlatt chapter, and was far easier to follow when translated into geonarrative form. This iterative shift in focus has not only created new possibilities for analysis and critical understanding, but has also helped shape ideas for additional geonarratives for inclusion in the Atlas (Brauen et al., 2011, 34).

Considering that one of the central goals of the atlas project was to incorporate Indigenous perspectives and knowledge, it is significant that the focus for the Treaties Module was suggested by an Anishinaabe individual, Alan Corbiere, and implemented; and, that the second mapping epiphany was precipitated by a mapping encounter with a Anishinaabe individual (Alan

\footnotetext{
25 The chapter by Debassige and Pyne (2012) is based primarily on a presentation made by Lewis Debassige at the Ojibwe Cultural Foundation's annual conference, Anishinaabewin Niizh, in February 2011. Months after the conference, Louis became and active contributor to the Atlas, speaking for seven hours about topics related to education policy, and telling many stories about community history and the evolution of Nation-to-Nation relations. Video clips from this talk can be viewed in the Travels in the Making of the Atlas Map at the point for June 9-15, 2011.
} 
Corbiere again) that involved the tracking of the Trickster, Nenboozhoo, in Anishinaabemowin, the Anishinaabe language. The decision made in January 2008 to geo-transcribe the Robinson Huron Treaty Survey Diaries is an example of intra-project iterative processes, in this case one map benefitting from a design insight inspired by participation in another mapping activity within the same atlas project.

The map creation process began from that evening and was followed by approximately three weeks of intensive research using a variety of sources to identify the approximate locations of the camp spots indicated in the survey diaries; in addition to transcribing the diary entries themselves. Sources included: the Dennis survey diaries for the 1851 and 1852 survey seasons (Dennis, 1851, 1853); Google Earth; the online Atlas of Canada; Michael Marlatt, who contributed background knowledge regarding survey techniques and instruments of the time and knowledge regarding locations; Patricia Kennedy, a senior archivist specializing in RG-10, the Indian Affairs file for Library and Archives Canada; and a diverse range of websites from the online Internet community, including First Nations community web sites. Since each point on the map could accommodate one place-based image, I was also keeping an eye out for relevant media as I searched the sites. I sent emails to the people whose images I had chosen for the maps, describing the mapping project and asking for permission to use their images. At the same time, I was learning about the history and geography of some of the places being mapped and planned to include some of this contextual information, ideally, as another map layer.

It is important to note that throughout the knowledge gathering process I had begun, I shared knowledge along the way, through talk and text, with all 
who were beginning to contribute in various ways. For example, in my letter to people whose media I had found on the Internet, I included a description of the Treaties Module project and some historical details related to the "survey story". I also referred to the educational aims of the project and the value of their contribution to the project. In this way, a working vision of the module including some of its contents and an idea of how they would be cartographically represented - was being created in people's imaginations (in their awareness) months before the Treaties Module was actually created.

\subsection{First Visit to the Lake Huron Treaty Region - An Adopted Homecoming}

Between February 14 and 22, 2008, I made a trip to the Lake Huron Treaty region to visit with a selection of community representatives. Although we had spent months previous to this visit engaging in telephone conversations, this would be the first face-to-face start toward working together. Much of the visit was devoted to explaining to people in further detail the vision for expanding the Treaties Module into an Atlas.

In its infancy, this project had already become near and dear to my heart, so leaving for this trip on February 14 was significant to me. Seven years prior to this, I had ventured into the Lake Huron Treaty region, but that time on a "personal" quest that made me very aware of the "power" of that land. Dispensing with the personal details, which I would share in a "friendship space", I can summarize to say that my repeated visits to Serpent River Traditional Territory - literally - at the turn of the century and the presence of the land were instrumental in my making significant life decisions that eventually led to my working on the Treaties Module. During that time, I actually 
approached someone from Serpent River First Nation and inquired about working for the Band. Although I had some valuable experience working as a transeditor for the House of Commons, it was apparent to me, and the person I was talking with, that the time was not yet right.

Thinking back on that time as I was driving, I was thankful for the way I was now able to give back. After seven years of living my new life in a way that allowed me to follow my heart and intuition, I was back.

As I drove, these thoughts came back to me in clear vividness, especially how I said that I would be back. And now, here I was - with more knowledge and experience, and a very interesting way to make a contribution - to give back. I describe this drive and all of the subsequent drives through the treaty region to visit with atlas collaborators as spirit drives. On these drives, I really get a chance to visit in the Ancestor space of reflection. As I have driven over some long stretches of highway through the Lake Huron region, with drives of up to 17 hours, I have always had plenty of time to reflect on the atlas project, its spirit, aims and direction; the meetings I would be having; conversations from the past, and such. The simplest way to describe it is 'reflecting with the land'. I mention these drives in part to highlight the importance of being on the land to any work that is about the land, and to draw attention to the performative nature of this work

As it turned out, there was a whiteout snowstorm, so I drove slowly, marveling at the beauty I was immersed in. Although it had been a beautiful experience driving slowly through the fluffy whiteout, it was a relief to arrive at North Bay in Nipissing Nation Traditional Territory around 8 p.m. Realizing the 
significance of media to the project - photography and video specifically - I took some photographs the next morning of Lake Nipissing.

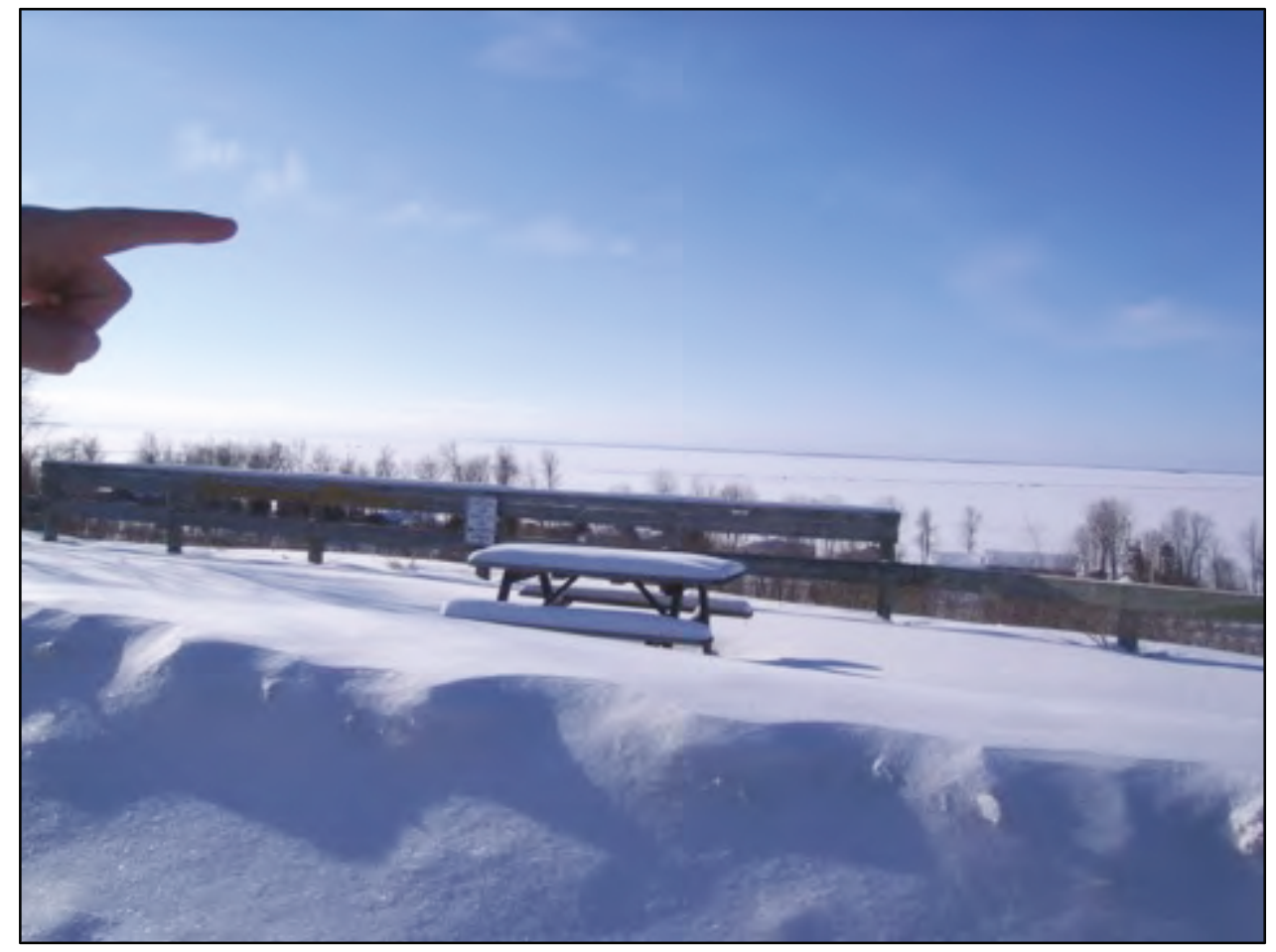

Figure 8.1. Looking out at Lake Nipissing with Daniel Gibson, morning, February 15, 2008 (photo by Stephanie Pyne).

On February 15, I headed to Dokis First Nation with my colleague Daniel

Gibson to meet with Gimaah Denise Restoule and Norm Dokis. Denise had recommended that Norm Dokis attend the meeting as well. Norm shared some interesting information about his Ancestor, Gimaah Michel Dokis who had signed the 1850 Robinson Huron Treaty on behalf of his community; and, Gimaah Denise told us about the recently published Dokis: Since Time Immemorial (2006), by Wayne Labelle, which includes may stories and photos of community members spanning at least 100 years. She later sent me a copy of the book and a DVD with a large selection of digital images from the book. These images remain a good resource for a potential Dokis Community History Map, which will allow 
people to make comments on the images, and upload related media. Although, I had originally intended to include some of the images from this book in the Treaties Module, the module did not have the scope to accommodate the images. Since the copyright statement at the beginning of the book recommended contacting individuals for permission to reproduce images they were featured in, I realized the necessity for more extensive community consultations in the initial stages of map creation involving the images from Dokis: Since Time Immemorial.

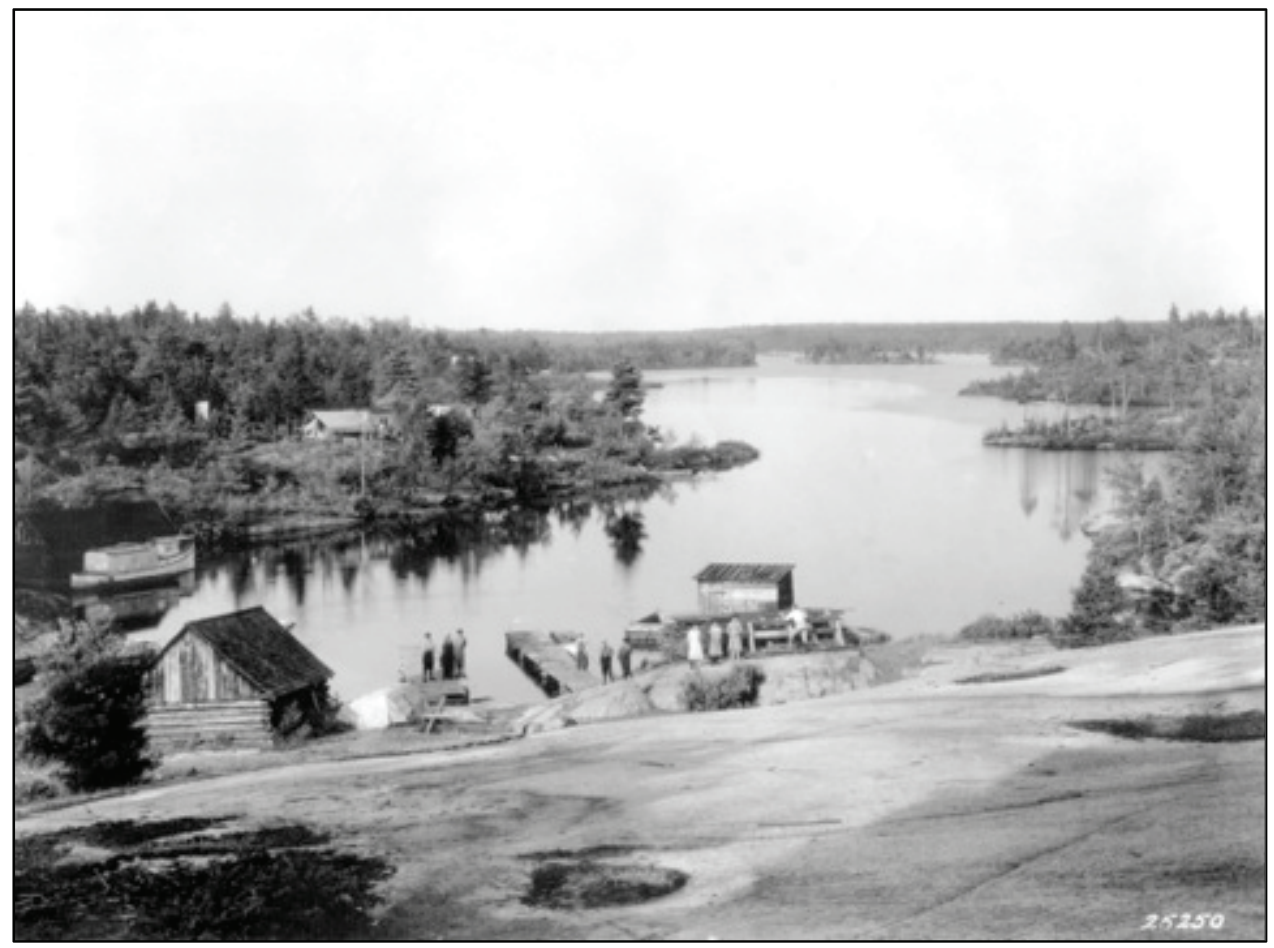

Figure 8.2. Photo from Dokis: Since Time Immemorial (2006) taken many years ago of the dock at Dokis First Nation.

Norm would later participate with me in phase II of the atlas project between October 20 and 23, 2009 in a mapping exercise that involved a boat trip on the French River in Dokis Territory. Our purpose: To retrace parts of the canoe journey of Surveyor J.S. Dennis between October 15 and 18, 1852, and to gather photographic media and locational information for eventual inclusion in the 
Atlas. The timing for this trip worked out well, being near the same time of the month and 157 years after the time that J.S. Dennis and a portion of his survey party were canoeing up and down the French River on Dokis Territory. The weather Norm and I experienced was very similar to the weather reported by Dennis in his survey diary (Dennis, 1852). Yet the many changes to the river and land due to the blasting associated with dam construction, and related infrastructure development initiatives, had obliterated the land in at least one location where Dennis would likely have camped while conducting the survey for the Dokis Reserve (Norm Dokis, personal communication, October 21, 2009; see Appendix D for the slideshow included at the point for October 20-23, 2009, Dokis First Nation, Travels of the Making of the Atlas Map).

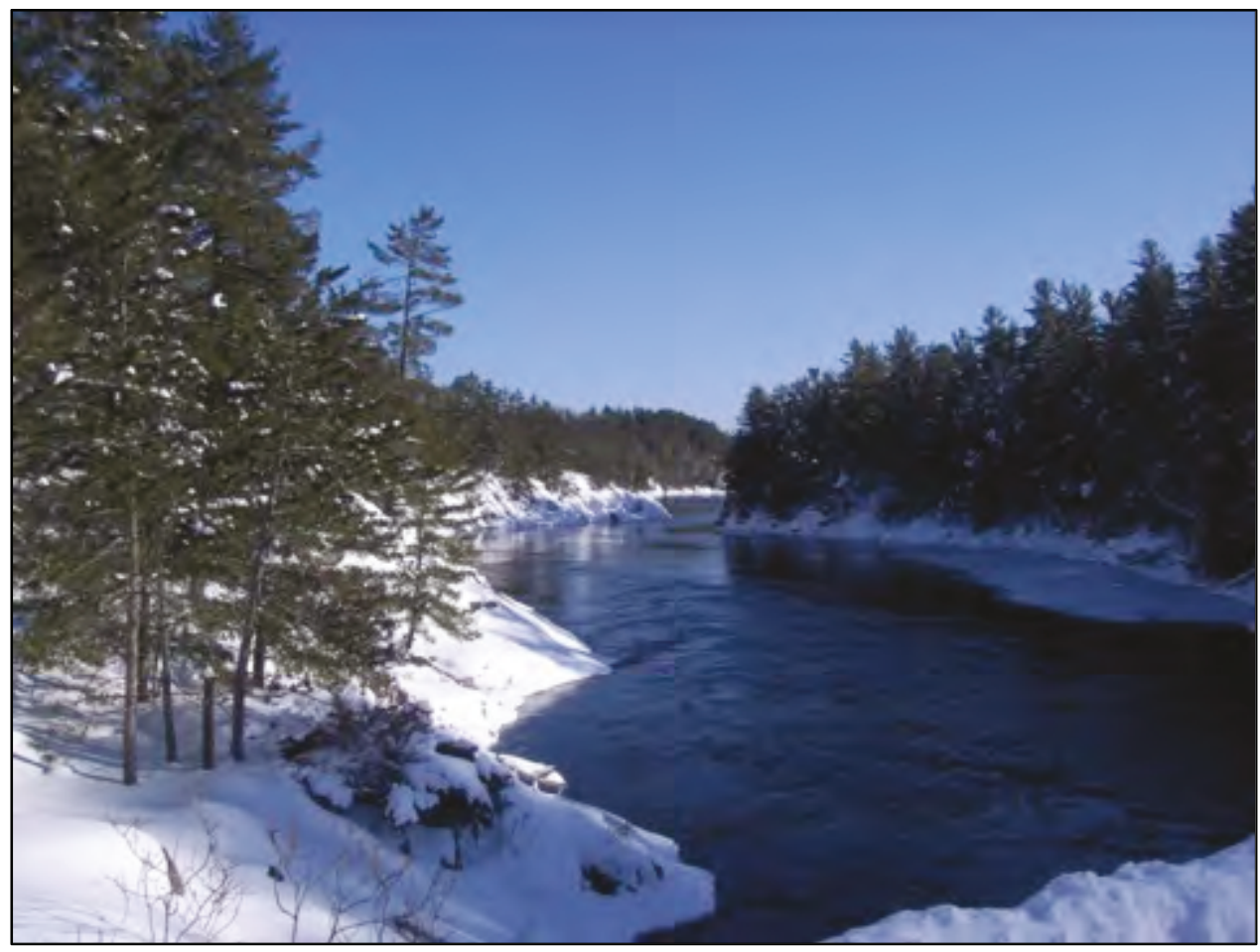

Figure 8.3. One branch of the French River on Dokis First Nation Territory, afternoon, February 15, 2008 (photo by Stephanie Pyne). 
On Saturday, February 16, Daniel and I met for the day with Gimaah Art Petahtegoose at Atikameksheng Anishnawbek (Whitefish Lake, Reserve No. 6). Art spoke at length about the importance of asserting Anishinaabe identity in his dealings as Gimaah with the Crown representatives and concluded with a discussion of the treaty history of his community. Of specific note in this regard is that Atikameksheng Anishnawbek was not surveyed during the Robinson Huron Surveys. A decision was made by lead surveyor J.S. Dennis not to survey Atikameksheng Anishnawbek and Wahnapitae (Reserve No. 11). Art agreed to allow us to video record his teachings, and we gave him tobacco as a gift reflecting our respect and gratitude, in addition to our acknowledgment of the guiding role of the Ancestors. The sample video clip of Art talking about the conceptual difference between chief and Gimaah is included in the Background section of the Treaties Module, and was carried forward during the subsequent phase II construction of the Lake Huron Treaty Atlas (see the point for Atikameksheng Anishnawbeg in the Nations under Robinson Treaties not Surveyed between 1851 and 1853 Map in the Lake Huron Treaty Atlas; also see Appendix D for sample video clip with Art Petahtegoose recounting the difficulties his Ancestors had following the failure of Surveyor Dennis to survey the reserved land for Atikameksheng Anishnawbek under the Robinson Huron Treaty). An understanding of this conceptual difference is central to gaining a clearer awareness of the intentions of the Anishinaabe signatories to the Robinson Huron Treaty, intentions that were neither faithfully nor accurately inscribed into the Treaty by translator and interpreter John William Keating. 


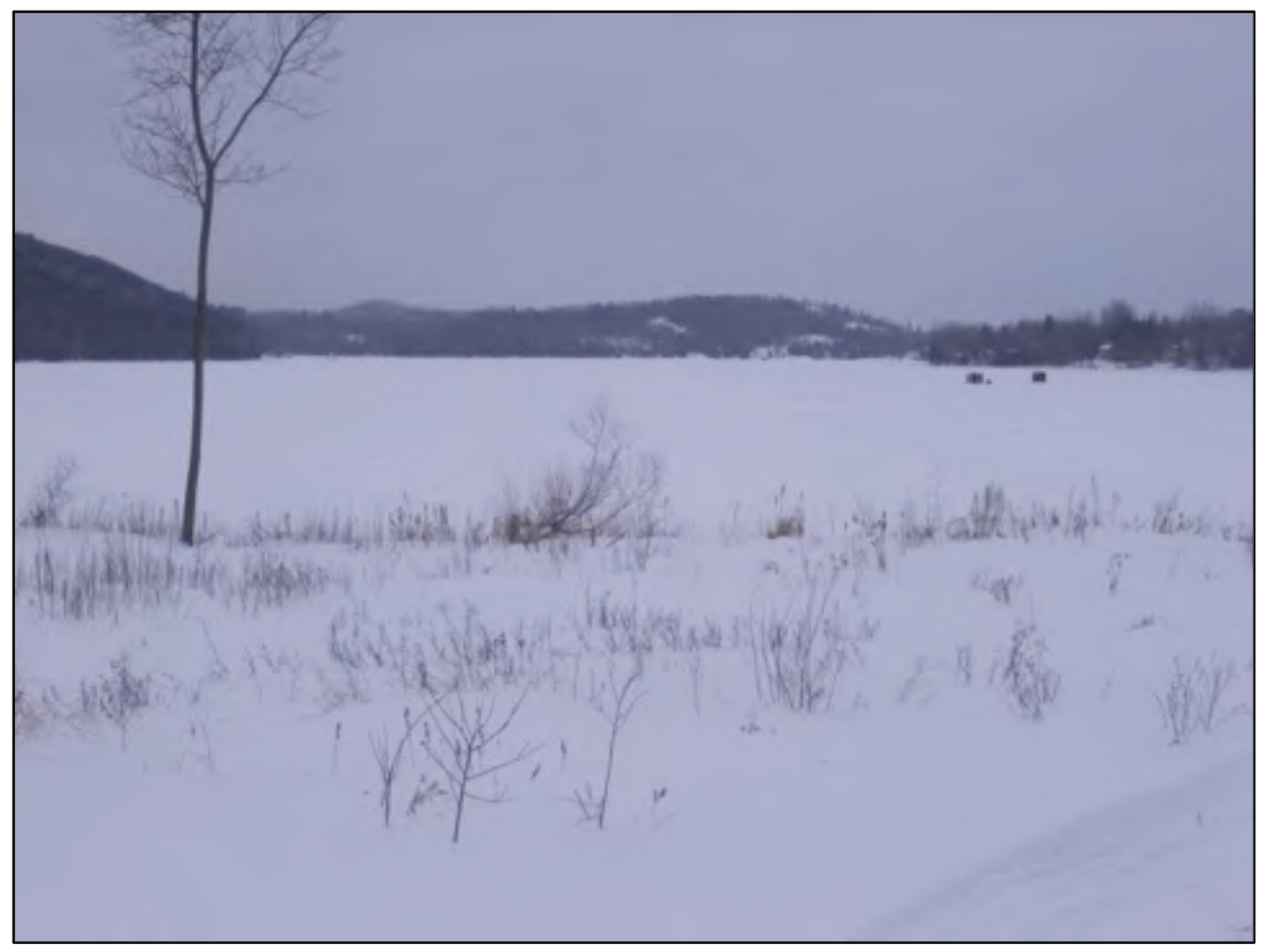

Figure 8.4. View of lake from Atikameksheng Anishnawbek Roadside, afternoon, February 16, 2008 (photo by Stephanie Pyne).

After our daylong meeting with Gimaah Art, Daniel left for Ottawa and I continued west to Manitoulin Island. After a rest on Sunday, I would go to the Ojibwe Cultural Foundation on Monday the 18th where I had been invited by Alan Corbiere to give a presentation to a group of Elders, including Gimaah Wilmer Noganosh from Magnetawan First Nation (Reserve No. 1) and M’Chigeeng Elder Lewis Debassige.

I arrived on Mnidoo Mnising (Manitoulin Island) in the evening under the cover of darkness and the brightness of the many stars that shone in the sky. It was full moon time, which added to the brilliance of the drive. The rocks along highway 6 on the way to the Island shone with a haloed glow beneath the glistening white fresh blanket of snow that draped down along the precipices. I 
opened my window and breathed in the crisp crystalline mist and introduced my self to the land.

The following Monday I had a chance to spend the entire day talking with the small group of Elders that Alan had invited. Alan informed me that that although Wilmer was from Magnetawan, his ancestor, Pamequonaishcung who signed the Robinson Huron Treaty on behalf of his community, was actually from M'Chigeeng and was living at Wikwemikong at the time of the treaty signing in 1850. The area around Magnetawan was actually the $\mathrm{M}^{\prime}$ Chigeeng people's sturgeon fishing grounds, which they would travel to at specific times of year, following the sturgeon spawning patterns.

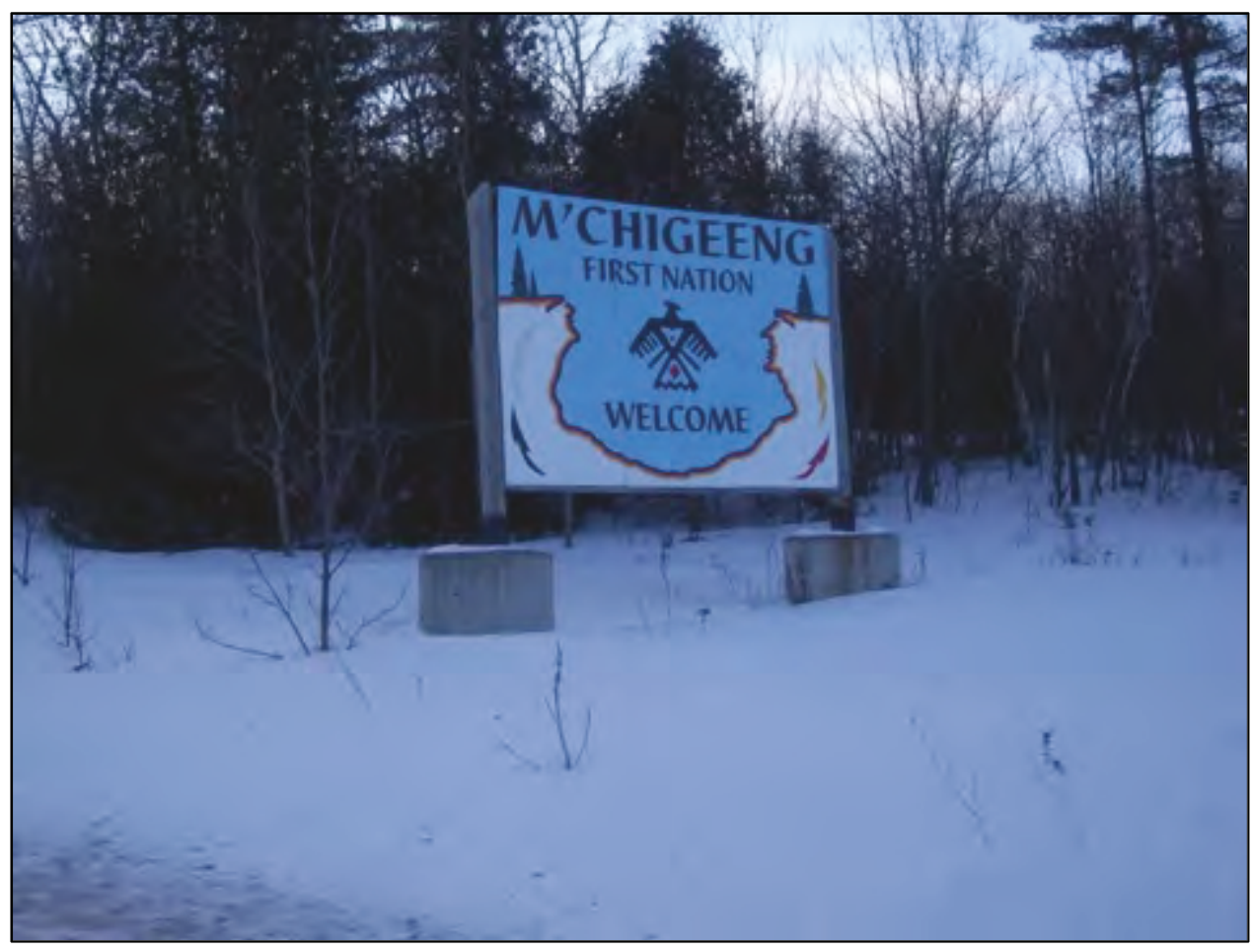

Figure 8.5. Entering $\mathrm{M}^{\prime}$ Chigeeng First Nation Territory, morning, February 18, 2013 (photo by Stephanie Pyne).

During the discussions, I shared a working conceptual design diagram that illustrated some of the significant dimensions that I envisioned should be 
reflected in both the Treaties Module, and the potential expanded atlas sometime in the future (see Figure 8.6).

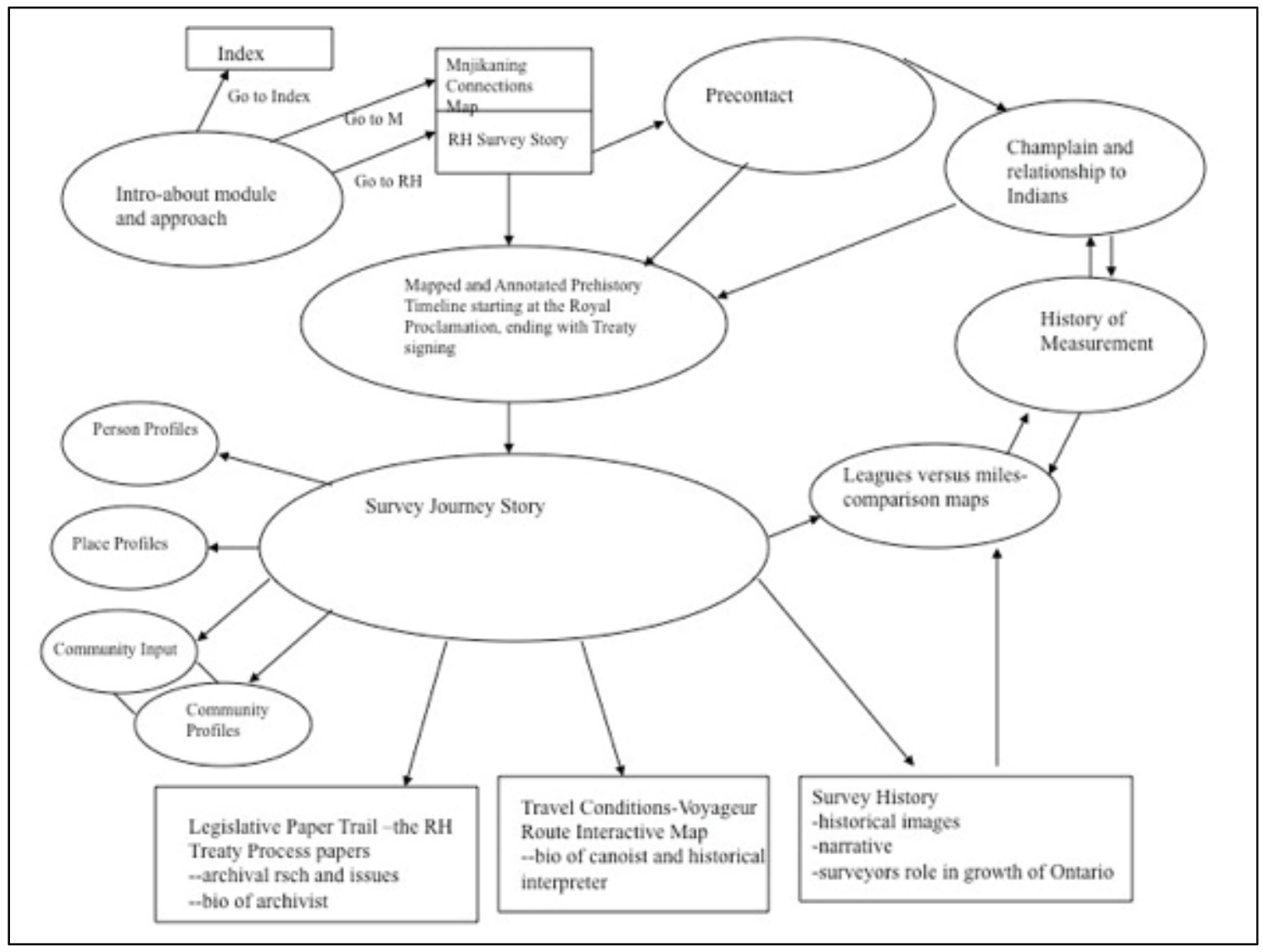

Figure 8.6. Initial conceptual design diagram for the Treaties Module allowing for potential expansion into an Atlas.

The next morning, I visited with Gimaah Shining Turtle of Wigwaasminising (Whitefish River First Nation, Reserve No. 4) on Birch Island, one of the stepping-stones from the mainland to Mnidoo Mnising. Gimaah Shining Turtle talked about how people used to navigate the shorelines by canoe, and about how the western boundary of the reserve surveyed by Dennis was curiously identical to the Wallace Mine eastern boundary. Since the Lands Commissioner was holding off on his approvals of mining lot leases until the 
reserve survey process was completed, factoring mining lot boundaries into the reserve surveys did seem to be putting the procedural cart before the horse.

We talked about the Treaties Module pilot project and its goal to contribute to a clearer understanding of the Anishinaabe signatories' intentions. After the meeting in Gimaah Shining Turtle's office, we went for a tour of the reserve. The Gimaah pointed out the reserve boundaries and emphasized how over the years the reserve had been whittled down even farther by a series of questionable "surrenders" to say the least, especially considering the lack of full disclosure and consultation with the Anishinaabe referred to in the surrender documents.

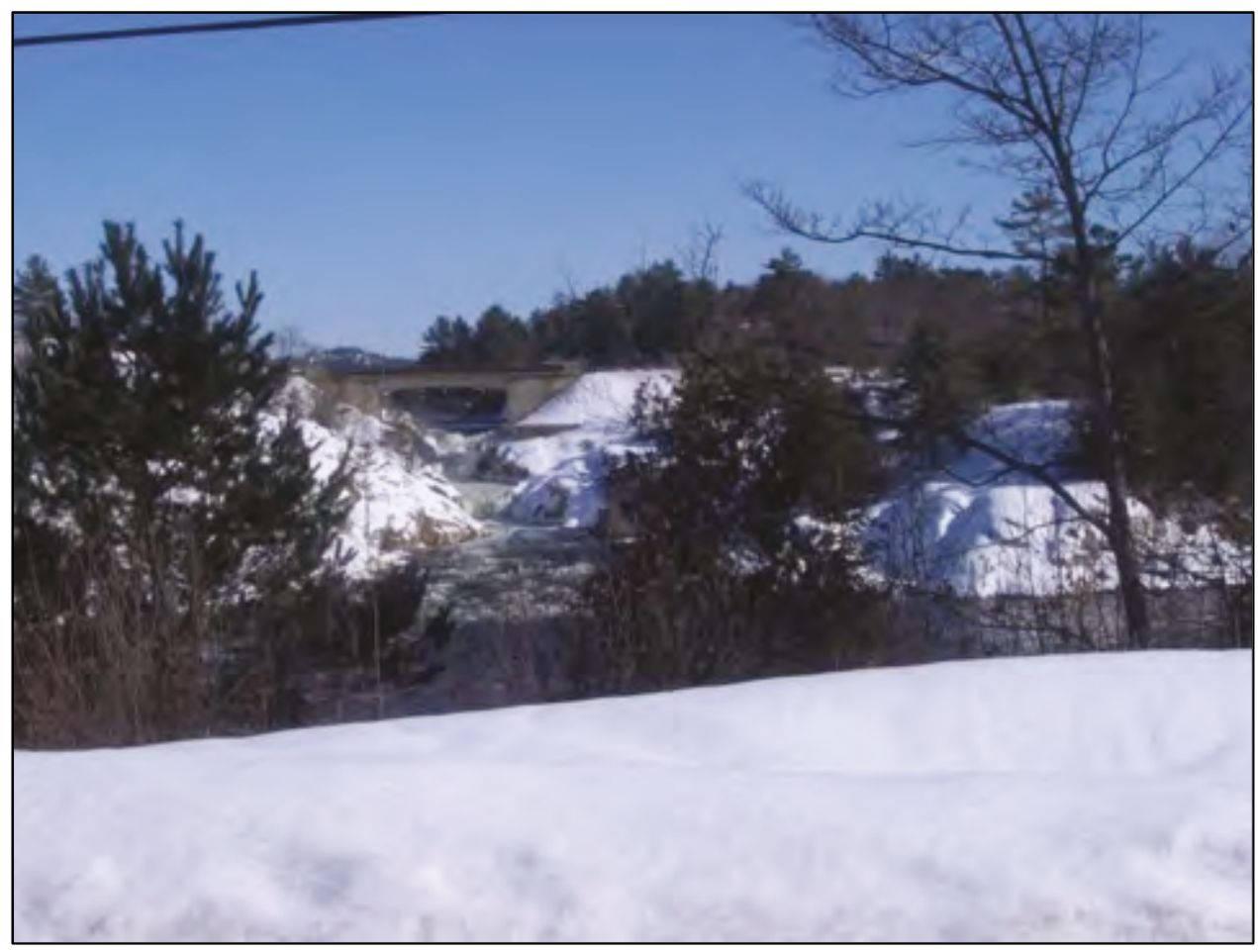

Figure 8.7. Whitefish Falls, one location that was, but no longer is, included within Wigwaasminising (Reserve No. 4) surveyed boundaries, morning, February 19, 2008 (photo by Stephanie Pyne).

Cartographically representing the evolution of the succession of surrenders over time for each Lake Huron Treaty Nation has remained a 
mapping agenda item for the Lake Huron Treaty Atlas since my early conversations with Gimaah Shining Turtle, and Alan Corbiere's initial request by email in July 2007. Following this, we visited the children at Shawanosowe where the Gimaah would be giving a talk to the grade sixes. He talked to the children about their spirit names and their clans, and the importance of discovering their own unique gifts through visioning. He also talked about Gimaah Wabakekik who had engaged in Lake Huron Treaty negotiations on behalf of his community, the importance of treaty history, and the significance of Wigwaasminising and Dreamer's Rock.

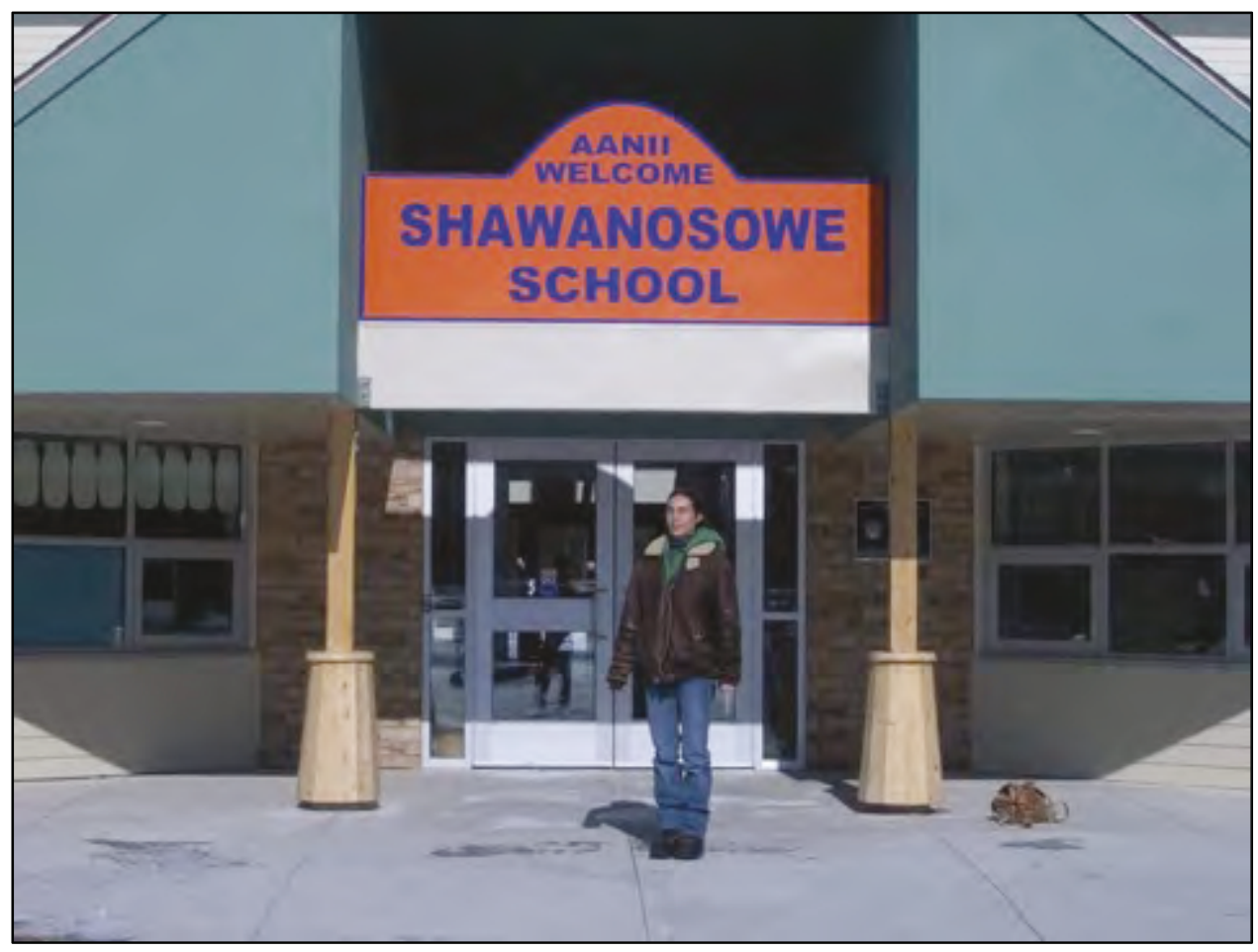

Figure 8.8. Stephanie Pyne in front of Shawanosowe School, February 19, 2008 (photo by Gimaah Shining Turtle).

After our meeting, Gimaah Shining Turtle sent me to visit the late Archie (Art) McGregor, who was a much loved and respected Elder along with his late wife, Violet McGregor. Archie, author of Wiiswaaskingaa (Land of the Birch Trees) 
(1999)", allowed me to audio record him as he told me stories about the Bell Rocks and Dreamer's Rock. The Bell Rocks were part of a sacred way the people of Wiiswaaskingaa communicated through the land to relations living great distances from them. Originally located on a large flat rock, the Bell Rocks sounded for at least 60 miles all around when struck. Archie told about the time when he was a young boy and had heard the Bell Rocks being sounded to signal the passing of the Gimaah of Sheguiandah (see Appendix D for digital recordings of Archie McGregor telling a personal story about the Bell Rocks; also available in the Community Stories Map of the Lake Huron Treaty Atlas at the point for the Bell Rocks on Big La Cloche Island near Wiiswaaskingaa). It is likely that the treaty signatories for Wiiswaaskingaa would have wanted this sacred location to be respected and protected. Instead, in 1972, a cement company removed the Bell Rocks from their flat rock platform and built a road over the platform. The company moved the Bell Rocks to another location on LaCloche Island, after which the Bell rocks ceased to sound (Archie McGregor, personal communication, February 19, 2008). 


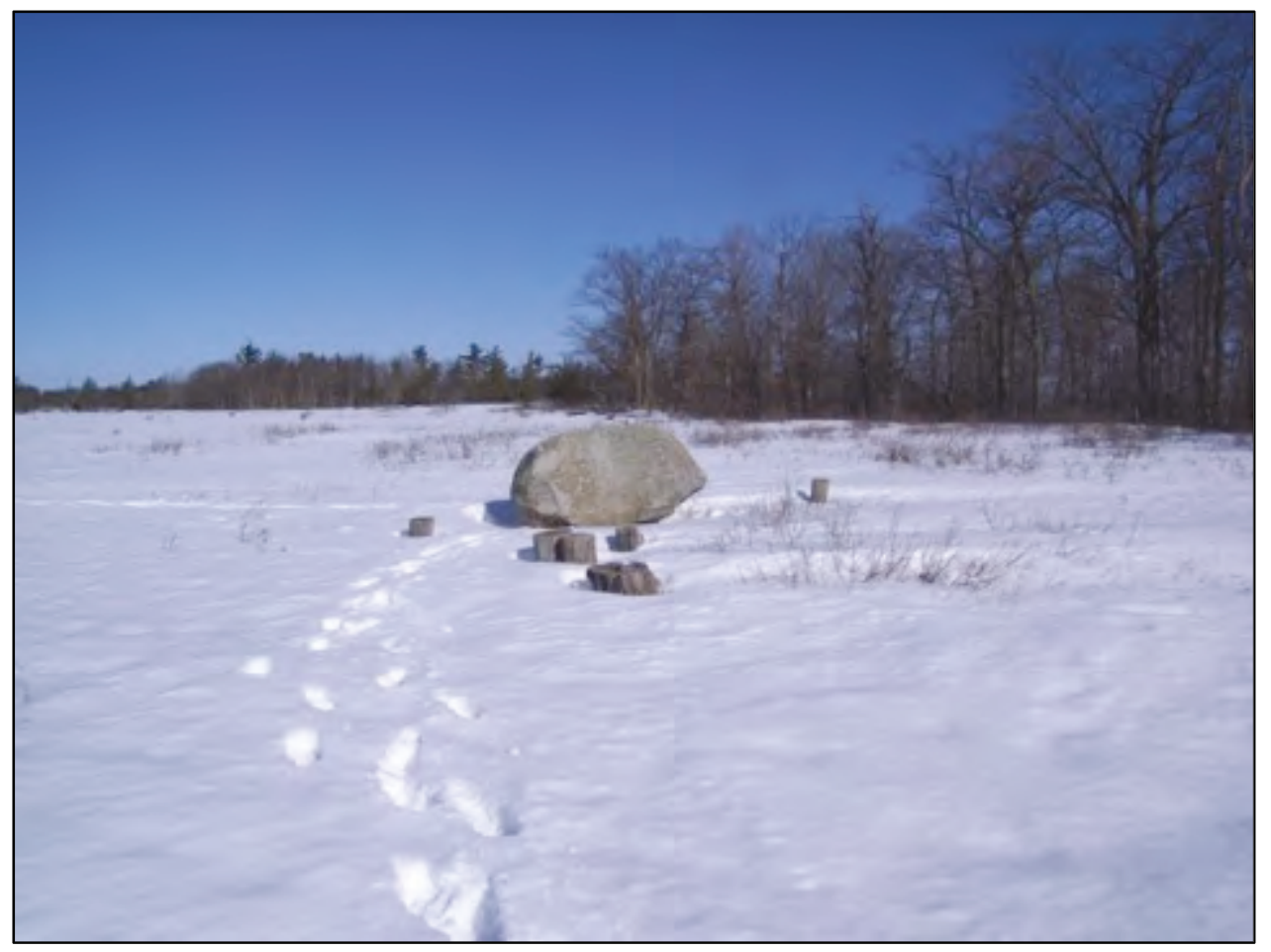

Figure 8.9 The new site of the Bell Rocks after the La Farge company removed them from the flat rock where they once stood, morning, February 19, 2008 (photo by Stephanie Pyne).

Hoping to include Archie's stories in the Treaties Module, I asked volunteer Atlas contributors Jesse Young and his friend, Dan Cummings to edit the audio, dividing it into sound clips and adjusting for sound quality. However, with the tight timeline of the project, the scope of the Treaties Module was narrowed to focus on the Survey Journeys Maps, and the Archie McGregor recordings would have to wait until phase II of the project, just prior to the launch of the Lake Huron Treaty Atlas in the spring of 2012. Prior to the phase II launch, I asked Perry Bebaamaash from M'Chigeeng First Nation to read the Anishinaabemowin parts in Archie's Wiiswaaskingaa (Land of the Birch Trees) (1999) that told of the Bells Rocks story and about Dreamer's Rock. We were communicating through Facebook, just one example of the important role social media has played in the Atlas development process. Perry did this in quick time 
and sent me the audio files electronically; I uploaded them to the Community Stories Map alongside with the edited clips of Archie telling the stories to me in English. I first met Perry in February 2010 at the OCF conference. He had seen the presentation I gave in Bawating (Sault Ste. Marie) talking about the Atlas vision. Sometimes it is clear immediately the form of contribution an atlas collaborator can make, and other times it takes awhile. Perry was studying Anishinaabemowin and teaching and wanted to teach the language in immersion programs. I saw Perry at a few different gatherings after first our first meeting at the OCF conference, and then kept in touch through Facebook, which has been a useful medium for collaborative interactions. Finally, when the pressure to produce the working Atlas at the end of Phase II was on, I asked Perry to read Archie's Anishinaabemowin text (see Appendix D for a digital recordings of Perry reading in Anishinaabemowin text from Archie McGregor's Wiiswaaskingaa (Land of the Birch Trees) (1999); also available in the Community Stories Map of the Lake Huron Treaty Atlas at the point for the Bell Rocks on Big La Cloche Island near Wiiswaaskingaa).

After my day visiting with Gimaah Shining Turtle and the late Archie McGregor, I left for Bawating (Sault Ste. Marie). I was looking forward to meeting the late Blaine Belleau with whom I had already had a number of insightful and instructive telephone conversations. Blaine was descended from Gimaah Shingwaukonse, the Robinson Huron Treaty signatory for Blaine's community, also an individual of great note (Chute, 1998).

In our conversation that day, Blaine talked about the contrast between the Anishinaabe concept of "share" and the British concept of surrender, and emphasized the fact that the treaty document, codified in English, does not reflect 
the Anishinaabe interpretation of "sharing" that was surely intended by the Anishinaabe signatories to the treaty (see Appendix D for a digital recording of Blaine Belleau discussing the difference between "surrender and share"; also at the point for Bawating, September 5-9, 1850 (the Robinson Treaties signing ceremonies) in the Marlatt Commentaries Map of the Lake Huron Treaties Atlas).

Blaine and I would continue to meet over the next five years, during which time, Blaine gladly volunteered to be video recorded sharing his views on many issues related to treaty history, interpretation and implementation, and emphasizing the importance of the need for mutual respect and fairness in nation-to-nation relationships between Gitigaan-ziibing and Canada. The understanding that began to develop during our first meeting in February 20, 2008 eventually flourished into the working History According to Blaine Belleau Map during phase II of the atlas project.

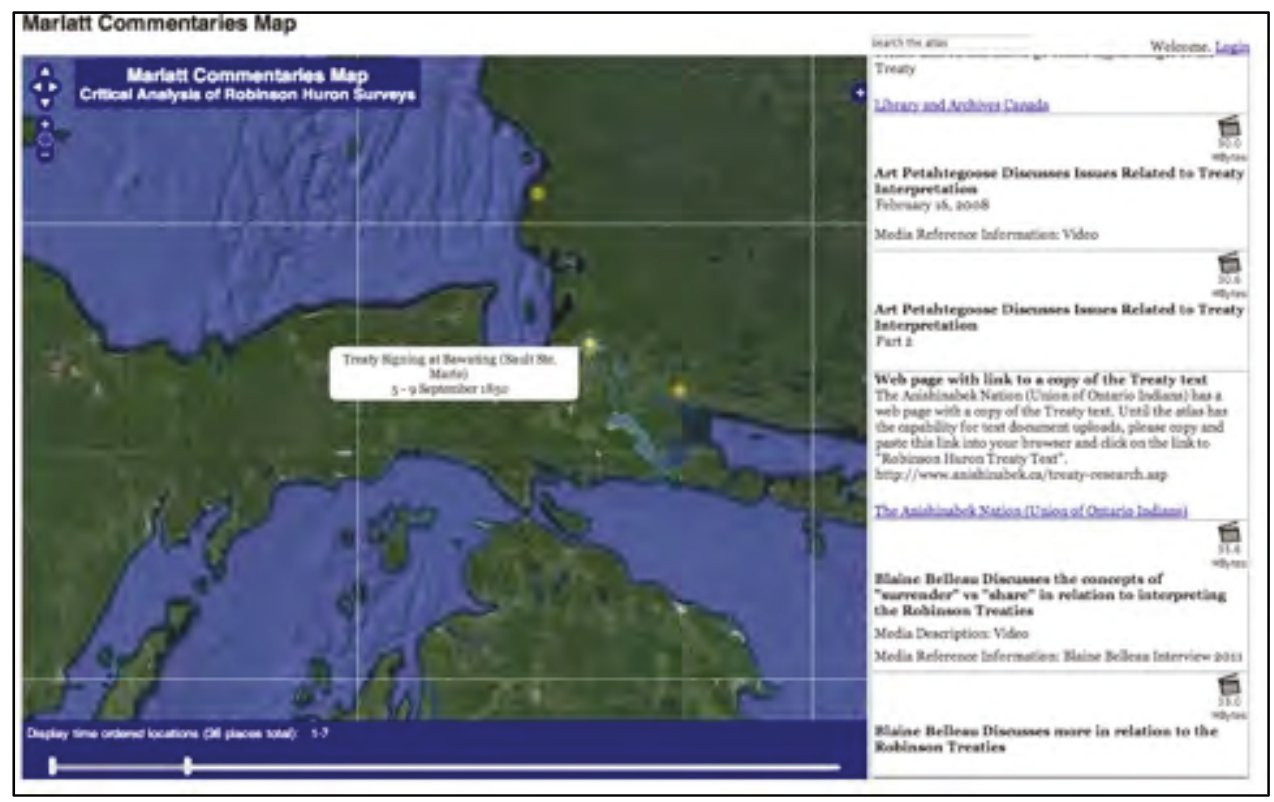

Figure 8.10. Screenshot showing point for Bawating, September 5-9, 1850, during the Robinson Treaties signing ceremonies in the Marlatt Commentaries Map of the Lake Huron Treaties Atlas. 
We finished our meeting with a tour of the reserve boundaries and a trip to the mouth of the Gitigaan-ziibing (Garden River), where surveyor Dennis had landed years ago in his survey canoe.

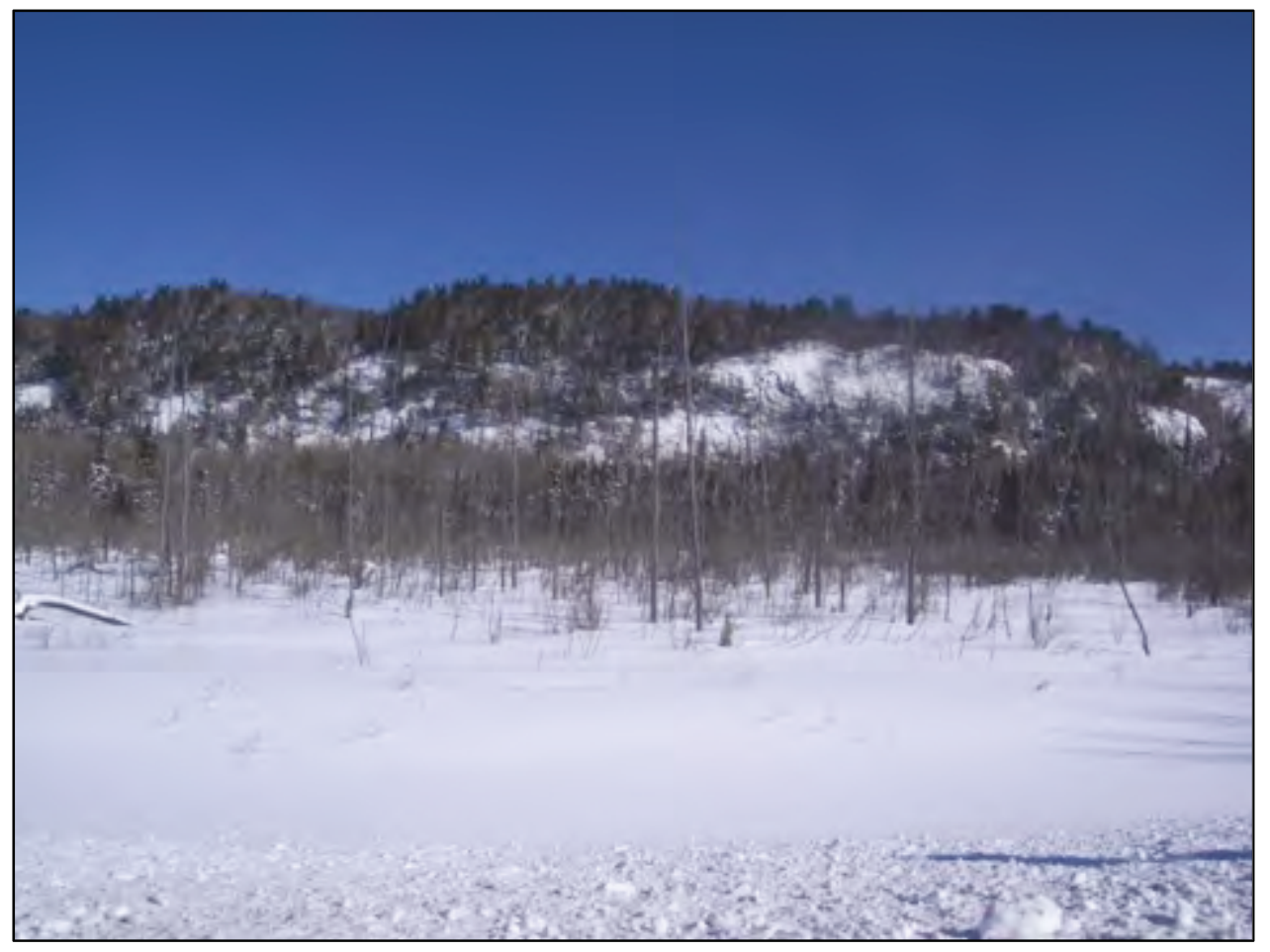

Figure 8.11. According to the late Blaine Belleau, Surveyor Dennis likely stood on this small mountain to make one of his readings, afternoon, February 20, 2008 (photo by Stephanie Pyne).

I left Bawating (Sault Ste. Marie) on February 21 around noon, after eating breakfast at the Husky. On my drive home, I decided to stop at Thessalon First Nation after lunch had a brief visit with Gimaah David Giguere. At one time, this was the biggest surveyed reserve. This was the place where the translator at the treaty signing (who was also participating as an "interpreter" in the survey process) admitted that he had erroneously translated the Anishinaabe term approximating the European concept for distance as "mile" when the Anishinaabe signatories had intended "league", a distance approximately 3.2 times the distance of a mile. In consequence, Dennis surveyed Thessalon in 
leagues, not miles; and the survey process took a correspondingly longer time to complete compared with the times taken to survey the other reserves, something I became acutely aware of during the mapping process to identify the approximate locations of the many camp spots Dennis occupied during that survey, since it took me a comparatively longer time to locate the Dennis party's many camp spots along the survey lines over a three week period. Today, Thessalon is the smallest reserve of all Robinson Treaty reserves, and will provide a particularly interesting study when it comes to mapping the evolution of land surrenders following the days when the Lake Huron Treaty was signed.

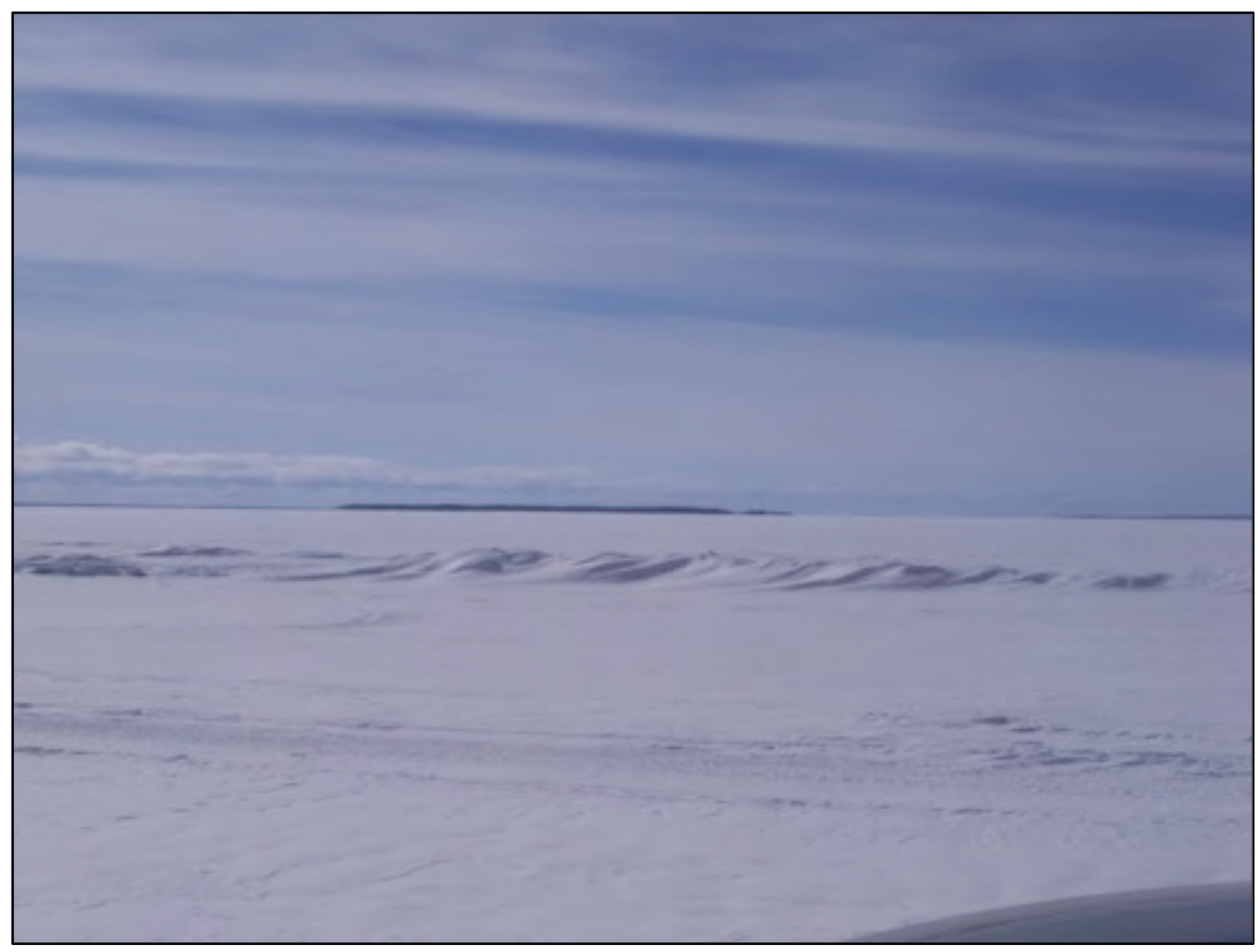

Figure 8.12. North Channel Lake Huron, afternoon, February 21, 2008 (photo by Stephanie Pyne).

These initial collaborative investigations contributed in a significant way. For example, at the meeting in M'Chigeeng to review the map prototype, Chief Wilmer Noganosh from Magnetawan First Nation "expressed concern about the 
use of "mouth of the river" as a geographical reference demarcating the territorial boundary, noting cultural differences in the interpretation of this feature" (Brauen et al., 2011, 28). This comment marked the birth of the idea to create a critical commentaries layer - a layer that would be available to all atlas users, providing the basis for additional comment and discussion. Although the idea for the critical comments layer was born from Wilmer's initial "critical" comment in phase I, work is still ongoing - especially along the technological and conceptual dimensions - to create the first critical comments map layer prototype (see Brauen et al., 2011 for a more detailed discussion of the iterative processes involved in work to create the critical comments layer).

I returned to Ottawa in the wee hours of the morning on February 22nd after a long but spirited drive, in which I took my time communing with the land as I flew through it.

\subsection{Work at the Lab - Winter 2008}

After I returned to Ottawa, I continued working on the construction of the Survey Journeys maps. Getting closer to atlas launch date, the weekly team meetings focused almost entirely on prototype creation. I had identified almost 100 approximate camp spot locations. I could not upload the information directly to a map, however, as I would later be able to do in 2009 after the conversion to the Nunaliit II software framework after the beginning of Phase II of the project. The Nunalit I software framework that supported the Treaties Module remained too complex. As we reported in Caquard et al. (2009):

Nunaliit, the software used to develop the atlas, is not yet at the point of enabling individuals without advanced computer skills to use it to create new modules. For instance, the design of the Nenboozhoo Mindemooye Story required the collaboration of both the community member who brought the story and members of the 
Geomatics and Cartographic Research Centre who had varying degrees of familiarity with Nunaliit. Indeed, Nunaliit still requires some coding in XML to develop content modules. Enhancing usability remains the major challenge for the future development of Nunaliit at this stage. While much effort has been put into successfully developing software that allows the stable rendering of geospatial stories in a multimedia format that is interactive and easily accessible from the user side, significant work remains to be done toward the goal of enabling any user to become familiar with the technology and easily develop her or his own module (96-7).

\subsection{The "Visiting the Circle - Enhancing the Relationship" Conference, Parry Sound, March 24, 2007}

Gimaah Wilmer Noganosh had invited me to attend this inter-cultural bridging conference, which was organized by the French River Visitor Centre Aboriginal Working Group and intended to educate people, including some provincial Ministry of Natural Resources employees, about Anishinaabe ways and worldviews. My son accompanied me and volunteered for the project by taking photographs of the land as we passed through it.

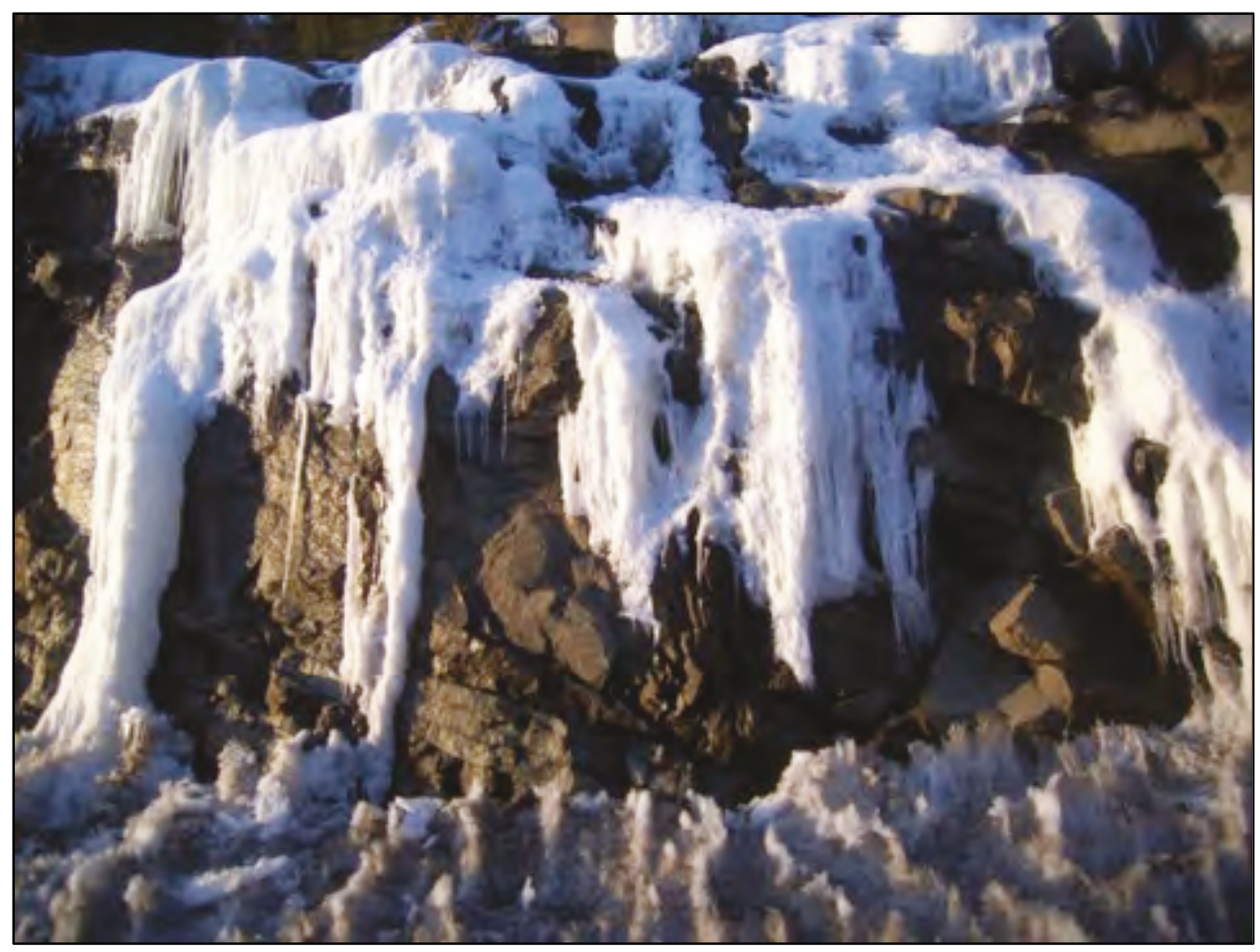


Figure 8.13. Driving through Algonquin Park on the way to the conference in Parry Sound, afternoon, March 23, 2008 (photo by Jesse Young).

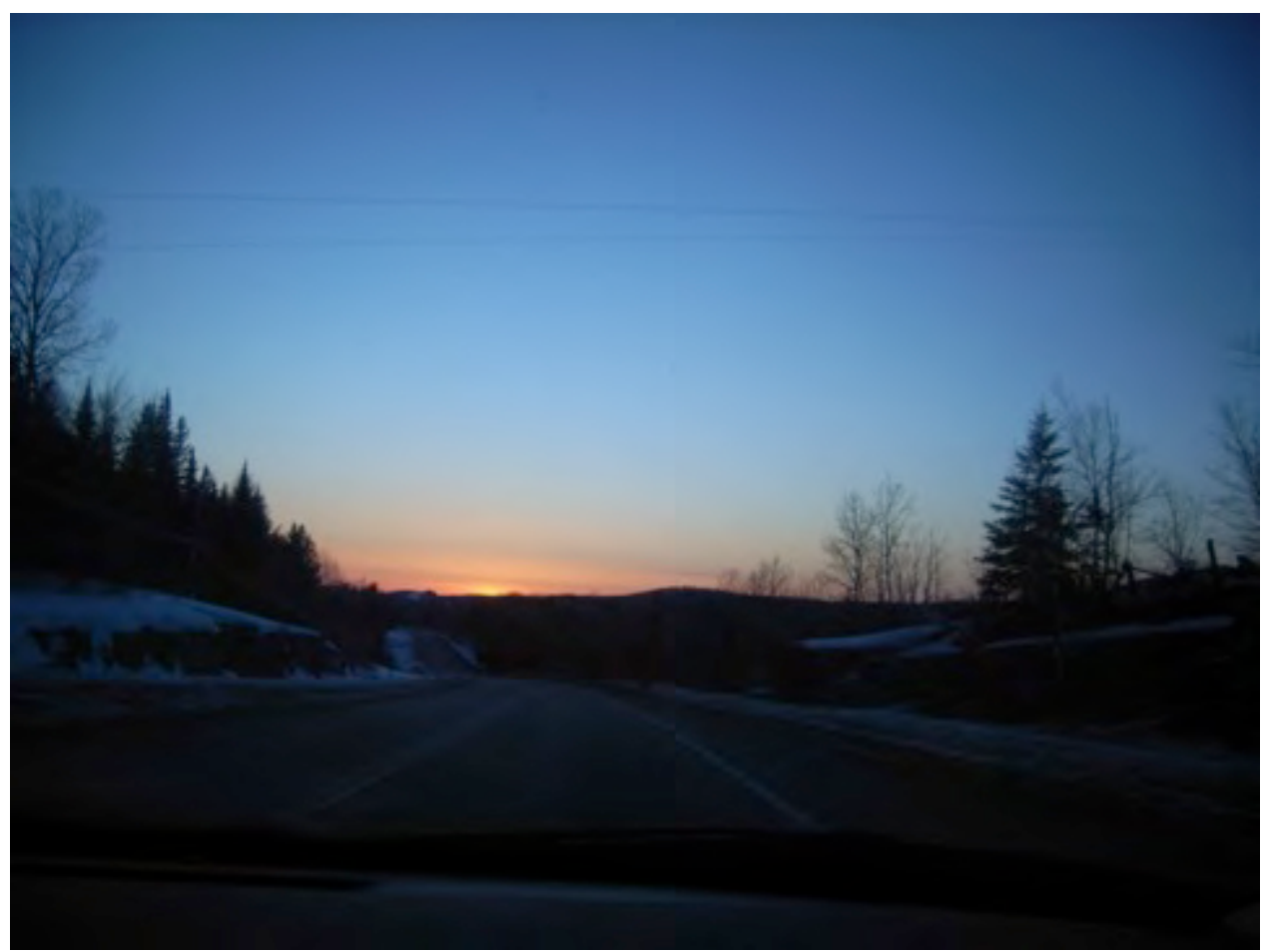

Figure 8.14. Driving through Algonquin Park on the way to the conference in Parry Sound, evening, March 23, 2008 (photo by Jesse Young).

We met Gimaah Wilmer when we arrived that same evening at the Comfort Inn in Parry Sound and helped out with setting up for the conference the next day. Among the people we met at the conference was Bill Allen. Bill had lived his entire life in the Lake Huron Treaty region, beginning in Thessalon and eventually moving to the Wausauksing area where he eventually became the principal of Parry Sound High School until his retirement; after which he returned to university and became a practicing archaeologist after graduation. Bill has described his approach as an interdisciplinary archaeology that integrates western methods with traditional Anishinaabe ways in the gathering and interpretation of artefacts and related historical geographical knowledge (Allen, 2002, 2006, 2010; Allen et. al, 2008). 
Bill was very interested in the project to create the Treaties Module as the basis for the broader vision, which has become Lake Huron Treaty Atlas; and in the month that followed our meeting at the conference, Bill sent me many photos he had taken of the Lake Huron Treaty region over the years, often including stories relating to them. During this time and afterwards, he shared insights and knowledge relating to the history and geography of the Lake Huron region via email and in telephone conversation; in addition to providing several papers he had written reporting on various archaeological findings in the region, and introducing me to several potential Atlas collaborators.

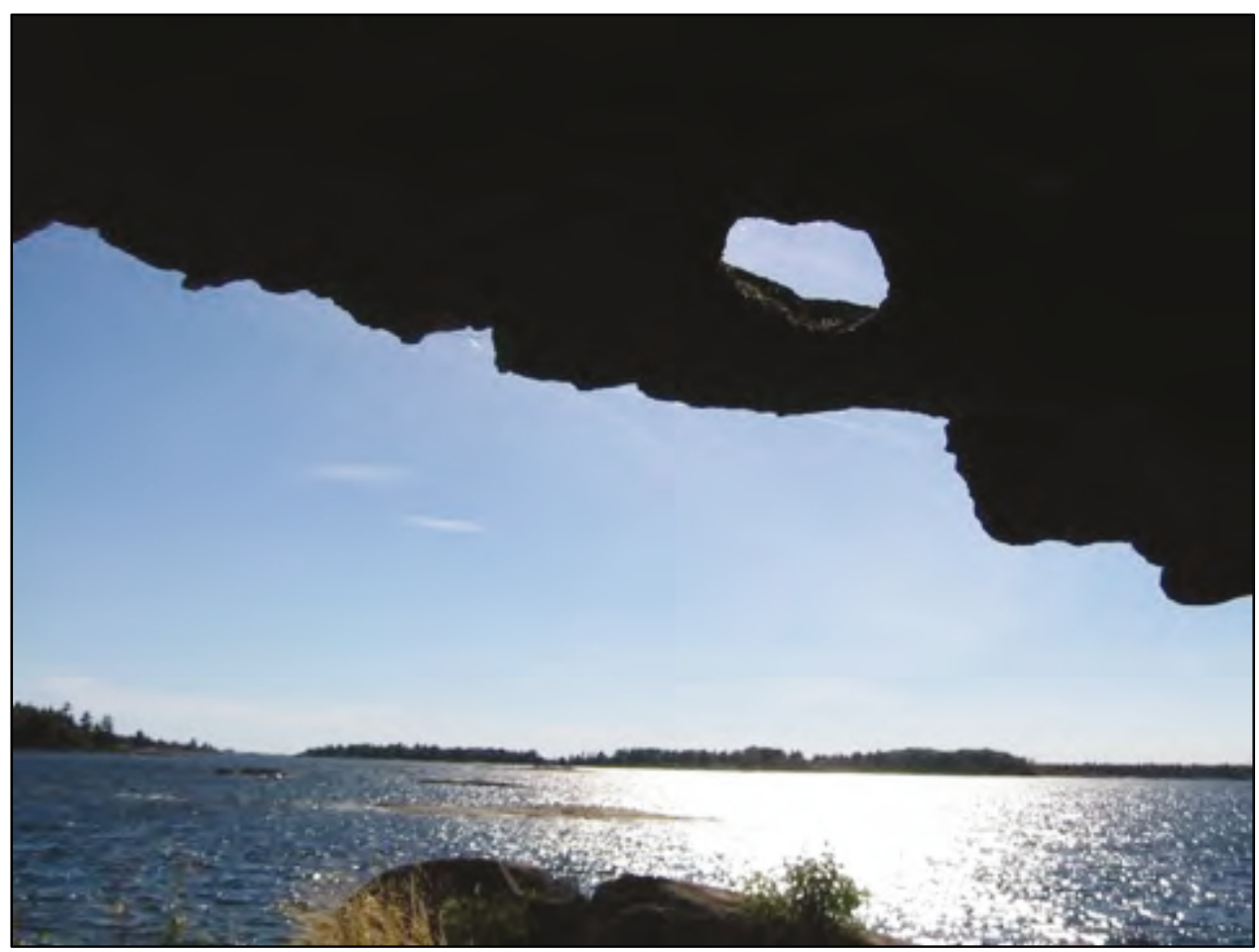

Figure 8.15. This sample of Bill Allen's photography is included in the Survey Journeys Maps. Looking toward Sandy Island (Isles aux Sables), the island that was originally referred to in the Robinson Huron Treaty reserve description for Wausauksing First Nation (photo by Bill Allen).

We also met Wilmer's cousin, Marilyn Capreol from Shawanaga First Nation, who I would meet several times later. Marilyn is involved in many 
cultural activities and knows the river systems and the Lake well, being an avid boater and fisherwoman. Conference presenters included youth and Elders, and the conference was an excellent opportunity for me to engage in some outreach and history sharing. Gimaah Noganosh talked to the group about bridging intercultural relationships and working together.

Jesse and I were not in Wausauksing Territory for long. When we left after the daylong conference had ended around three o'clock, a colossal snowstorm had started. It was beautiful but challenging driving.

\subsection{Work at the Lab - Pre-launch "Festivities"}

We returned to Ottawa late in the evening and I continued working on the Treaties Module, with the launch only a month away.

Having a practical deadline objective forced certain issues to come to the surface and gave rise to some innovative ways to present information. The month preceding the atlas launch involved very long working hours for me. Part of this was related to the fact that I had been gathering stories and Treaties Modulerelated contributions with the bigger Atlas picture down the road vision, so I had more content than I needed for the prototype Treaties Module.

I involved my family once more. My son Jesse and his friend Dan Cummings edited the Archie McGregor audio recordings, and the recordings my brother, Jason Pyne. Jason had recorded his voice reading the J.S. Dennis diary entries, which are included in the Survey Journeys maps, making him effectively the 'voice of Dennis'. In addition, my good friend the late Murray Stephens, transcribed some of Dennis' field notes, which remain on the agenda for inclusion in the Atlas. As I mentioned above, the Archie McGregor recordings were added to the Atlas in Phase II of the atlas project. In addition to the work 
involved in rendering the content I had gathered into cybercartographic geonarrative form, I was responsible for making arrangements for out of town atlas launch guests, including Blaine Belleau, Alan Corbiere, Wilmer Noganosh, and Bill Allen.

\subsection{Community Launch at Shawanosowe School, April 23, 2008}

After a month of 12-14 hour days and 8 hours of work in the lab on the last day of the pre-launch phase of the project, I left around 7 p.m. on April 22 for Magnetawan. I was going to pick up Gimaah Wilmer Noganosh who was going with me to the community launch presentation at Shawanosowe School in Wigwaasminising (Whitefish River First Nation, Reserve No. 4). I arrived in Magnetawan at 2 a.m., and began driving again around 7 a.m. heading to Wigwaasminising. We arrived at around 10 a.m. I was a little on the tired side, but I was happy and excited to be here with the kids. I was also a little nervous to speak.

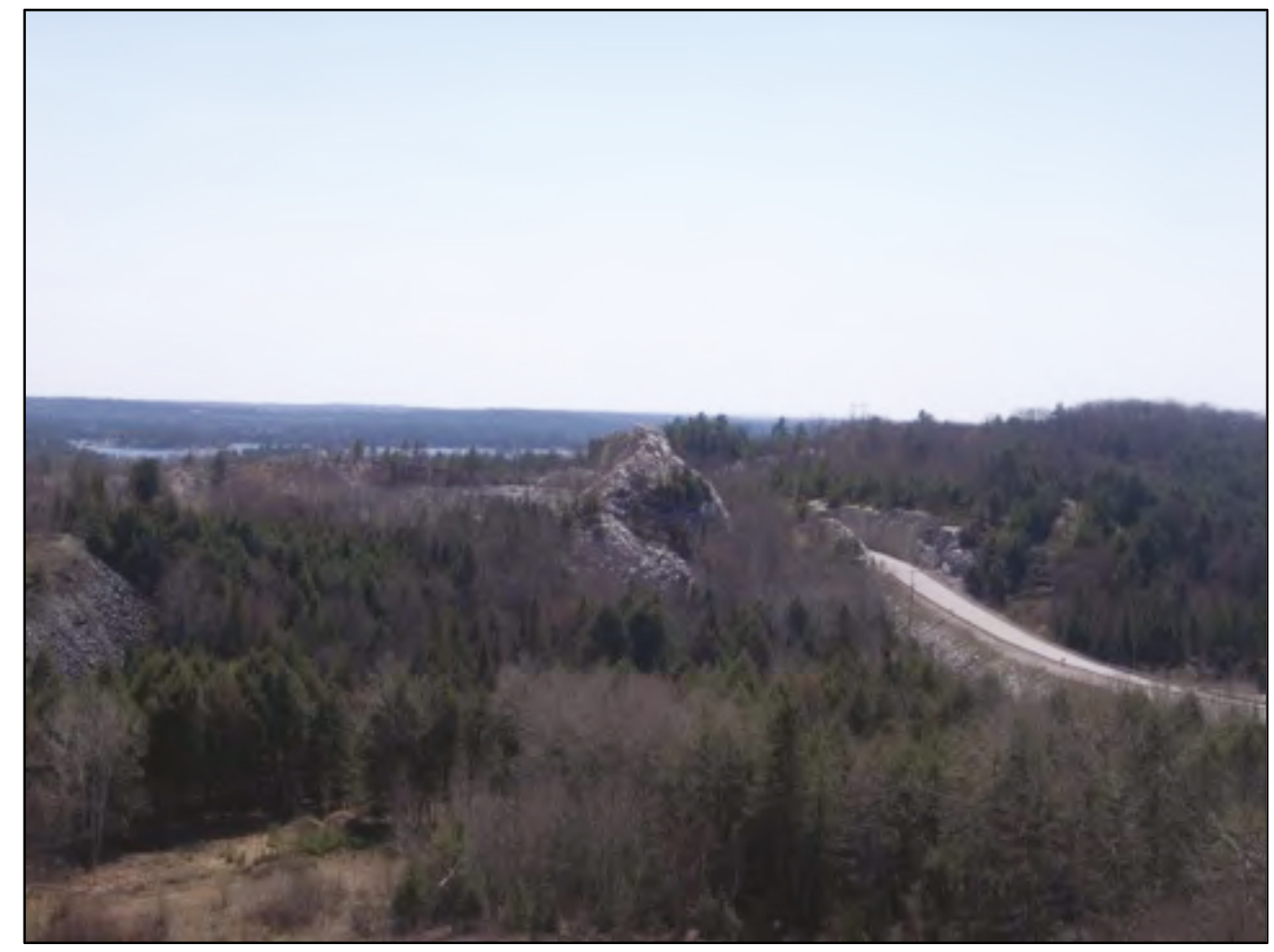


Figure 8.16. Looking south toward Wigwaasminising, afternoon, April 23, 2008 (photo by Stephanie Pyne).

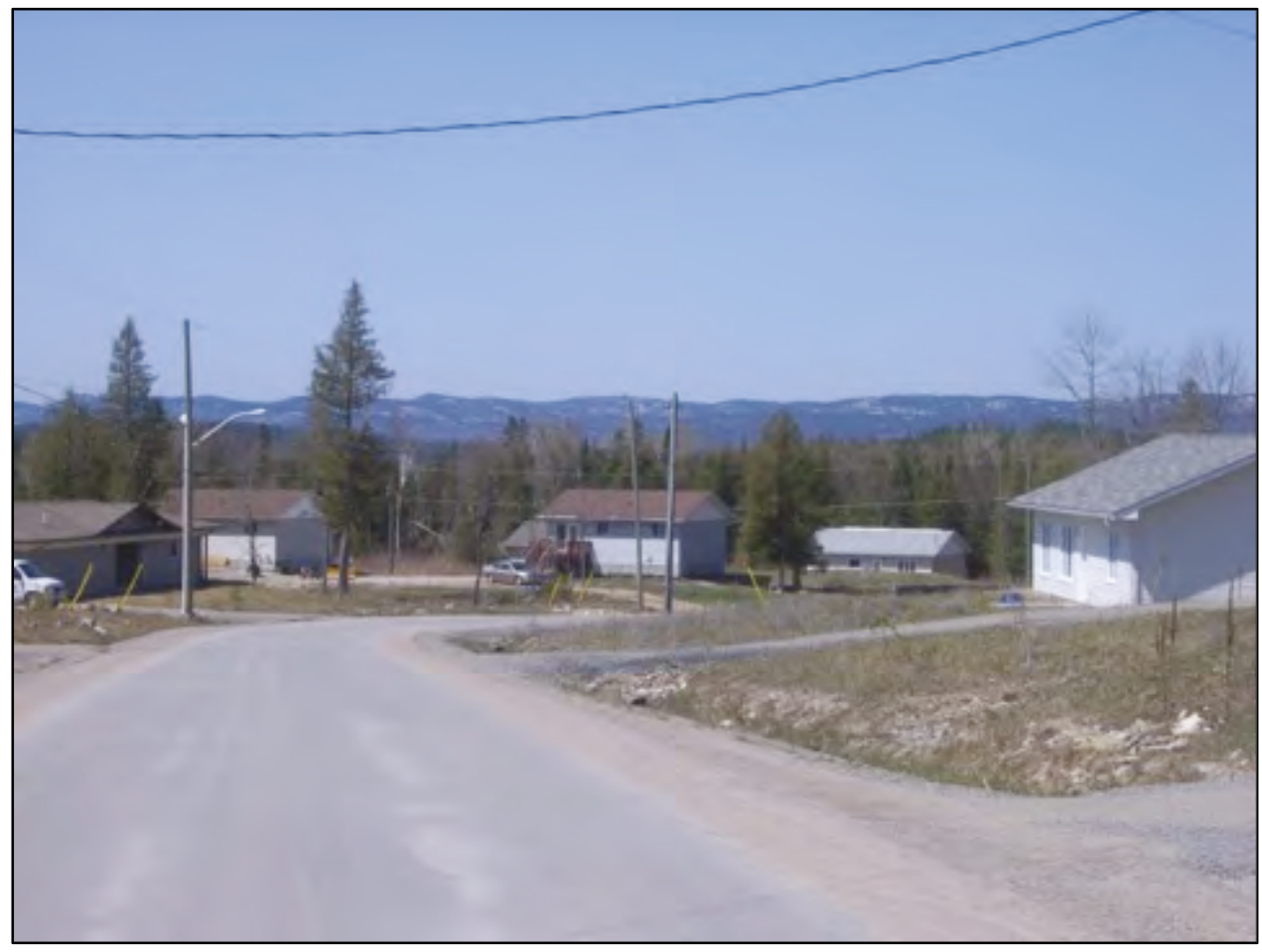

Figure 8.17. Looking north toward the LaCloche Mountains from Wigwaasminising, afternoon, April 23, 2008 (photo by Stephanie Pyne).

I planned to tell the grades fives and sixes the "Rainbow Raven and the Fly in the Bottle" story, which makes up the Spirit section of the Treaties Module. I had brought five acrylic paintings on canvas, which were created and later digitized to graphically interpret the story in the Treaties Module. Before the discussion began, I asked a group of children to take the paintings and arrange them in the order they thought they might go in. To my surprise, the children arranged the paintings in the right order (after having a great time running around the gym with them). When I told them they had got it right, and when I asked them how they knew the order of the paintings for a story they had not yet heard, the children replied that they had heard that story in Ojibwe class. Although the story of Rainbow Raven and the Fly in the Bottle has different 
origins from the Ojibwe story recalled by the children, the themes and directions of the two stories were sufficiently similar that the children were able to enact both stories by arranging a series of artworks, which - apparently - told a story in themselves.

Once the presentation was over, Wilmer and I went to Whitefish Falls, which used to be within the surveyed boundaries of Wigwaasminising. We also went to Willisville, which Archie McGregor had mentioned when he talked about the land that used to belong to Wigwaasminising. Then we drove to Ottawa for the launch, and arrived just in time for its start at 7 p.m. that evening, just 24 hours after I had left Ottawa for Magnetawan.

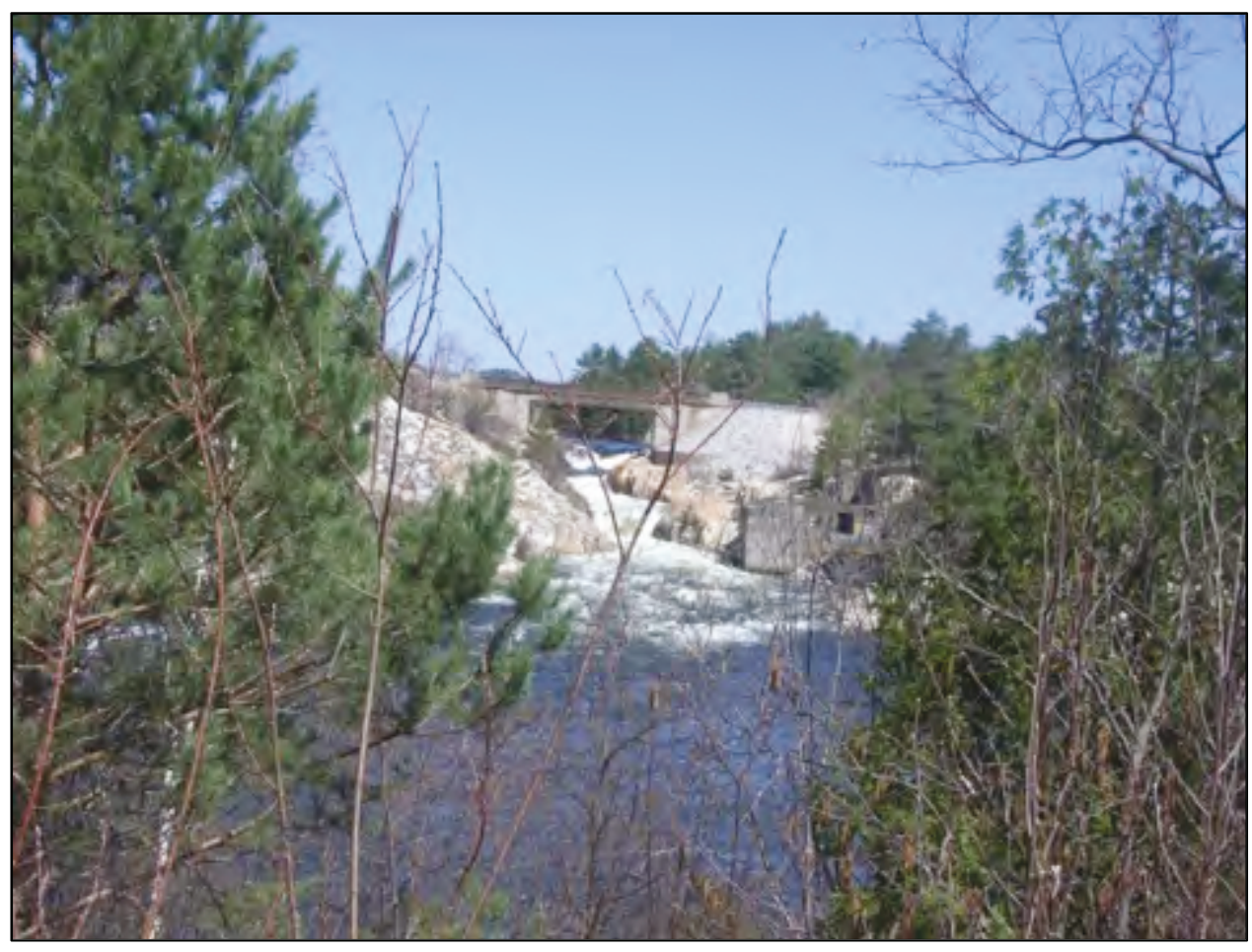

Figure 8.18. Whitefish Falls, afternoon, April 23, 2008 (photo by Stephanie Pyne). 


\subsection{The Ottawa launch of the Fledgling Atlas of Indigenous Perspectives and Knowledge}

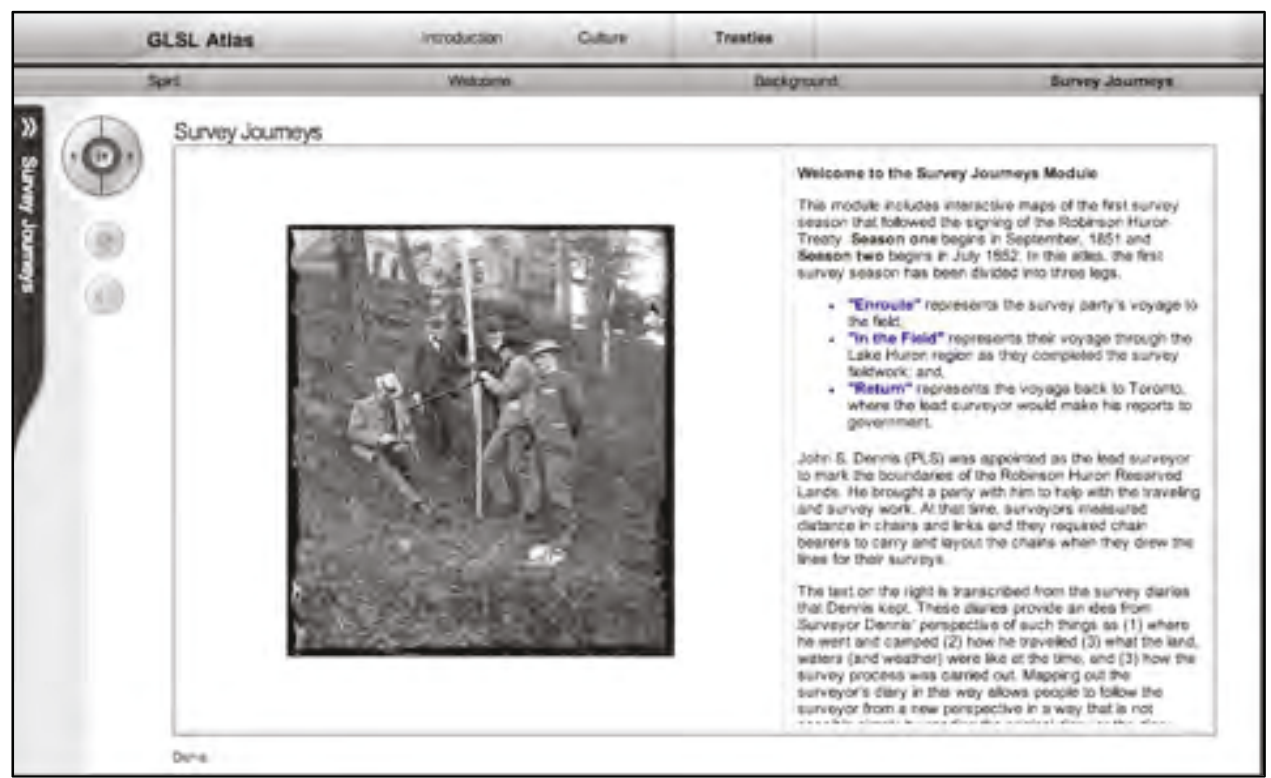

Figure 8.19. Screenshot showing first pane of Survey Journeys Maps in the Treaties Module.

We were honored to have Grandfather William Commanda and Romola Trebilcock attend the launch, and I am thankful to Bill Allen for introducing me to Romola Trebilcock to whom I made my request for their attendance. When William and Romola arrived at the lab for the Atlas launch, I gave William tobacco for his blessing of the project, which I envisioned to continue for many years to come. He said, "That's a lot of tobacco and smiled". I replied, "There's a lot of work to do. It needs all the help it can get". 


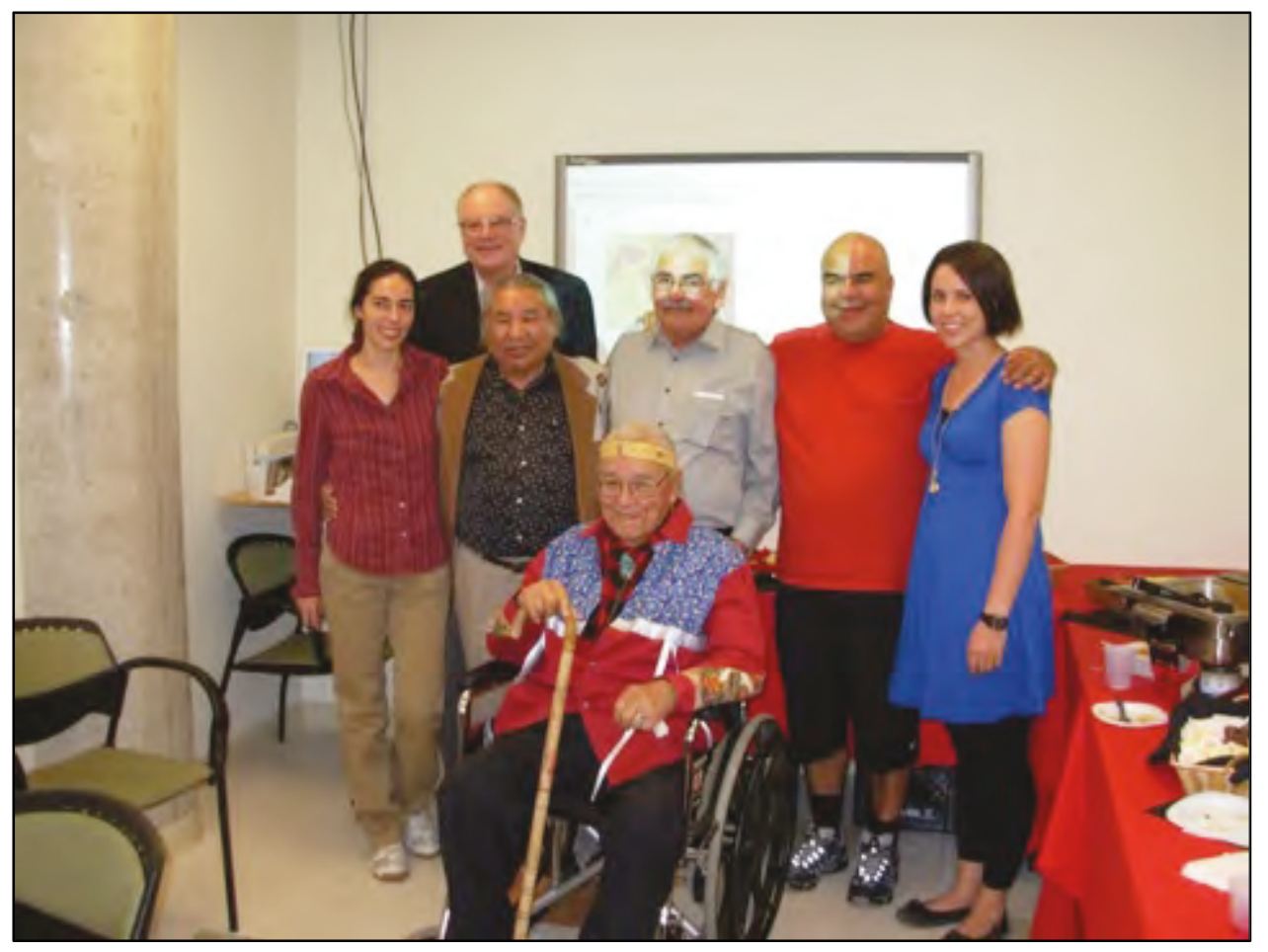

Figure 8.20. Group photo of some Atlas contributors and launch guests, evening, April 23, 2008. Front: late Dr. William Commanda Second Row (left to right: Stephanie Pyne, Gimaah Wilmer Noganosh, Magnetawan First Nation, the late Blaine Belleau (Gitigaan-ziibing or Garden River First Nation), Brad Henry and Christina Moore (People of the Longhouse). Back Row: Bill Allen (photo by Alan Corbiere). 


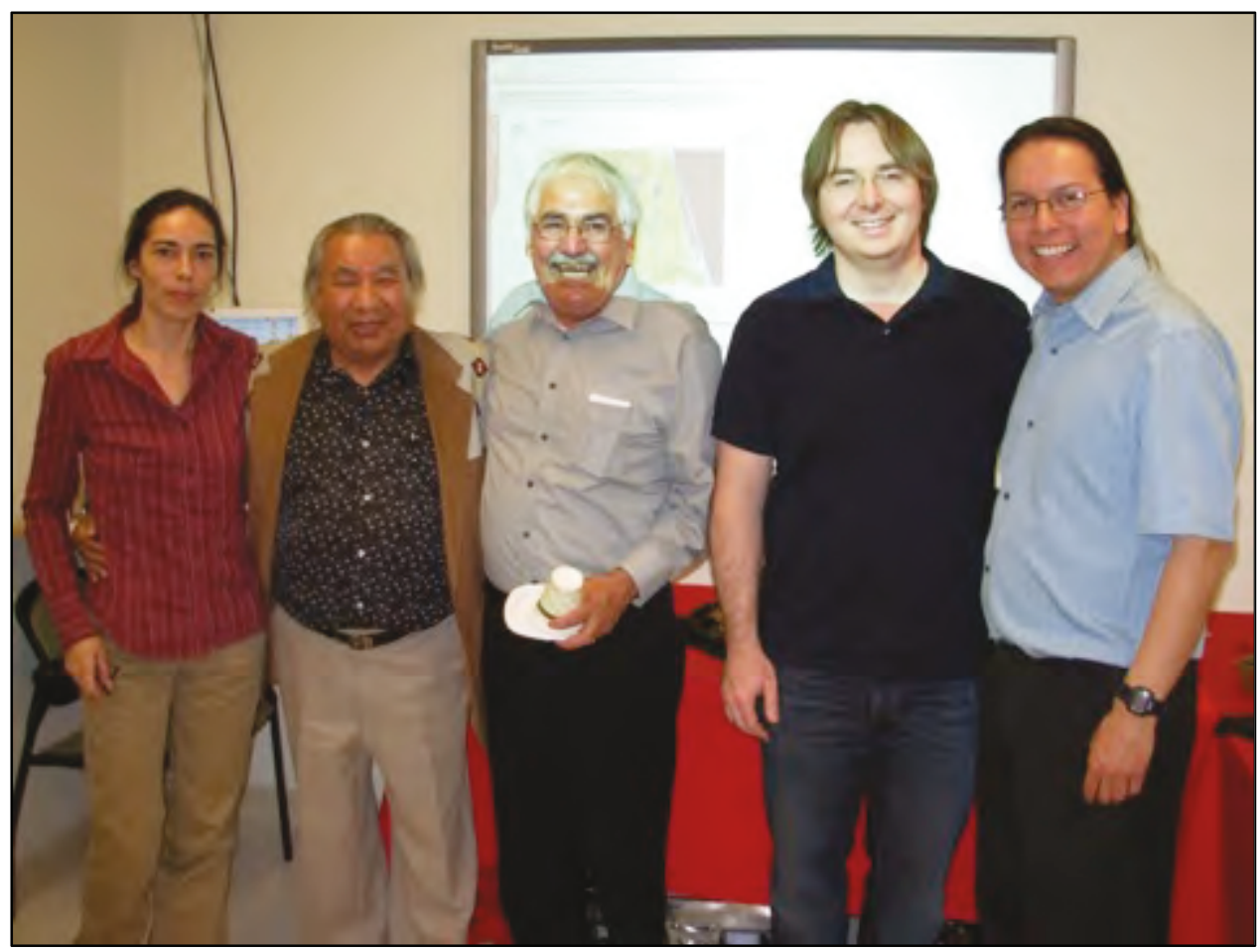

Figure 8.21. Group photo of some Atlas contributors and launch guests, evening, April 23, 2008. Left to right: Stephanie Pyne, Gimaah Wilmer Noganosh, Magnetawan First Nation, the late Blaine Belleau (Gitigaan-ziibing (Garden River First Nation), Amos Hayes, and Alan Corbiere (photo by Bill Allen).

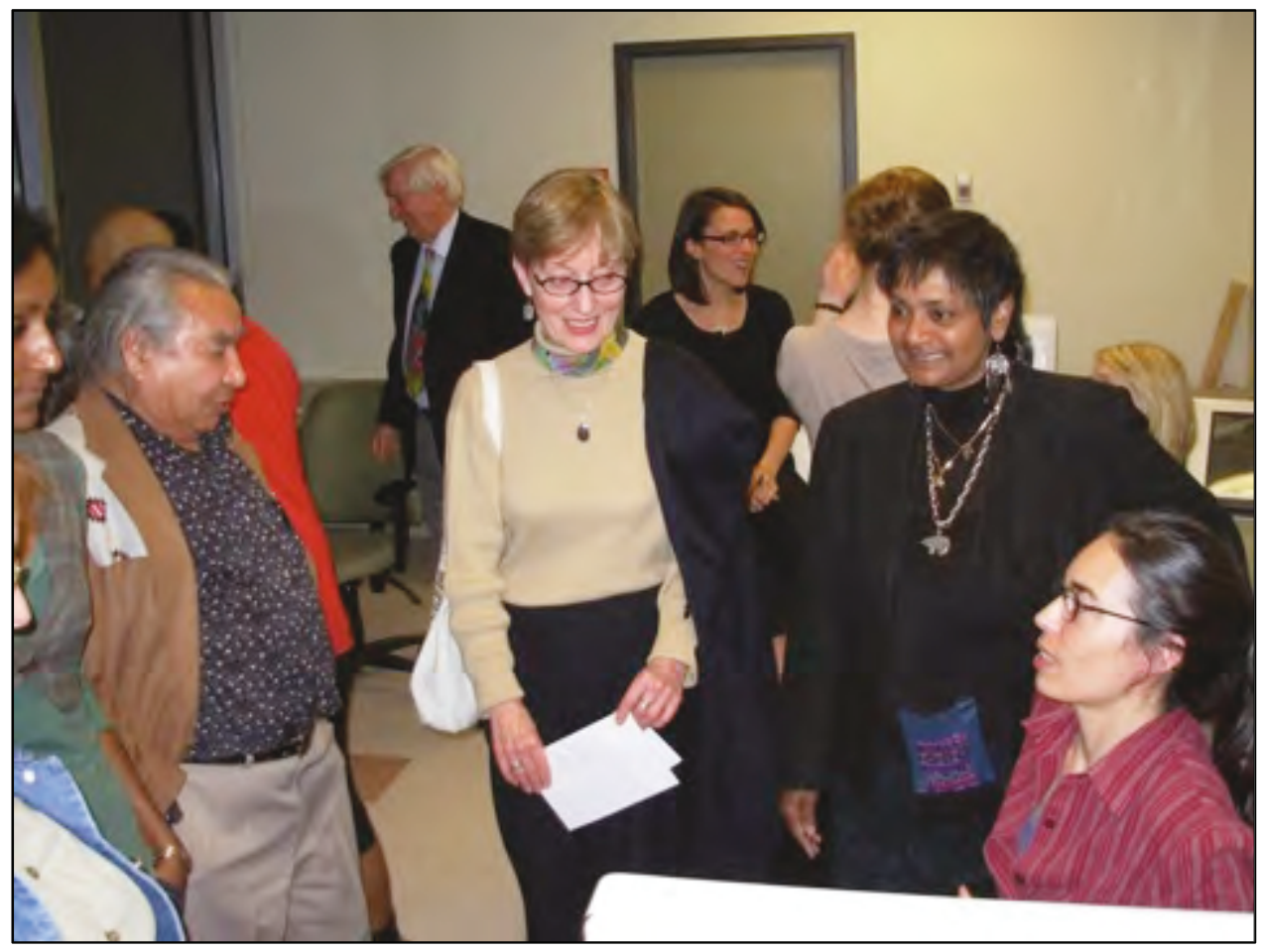


Figure 8.22. Atlas demonstrations and discussions (photo by Alan Corbiere).

\subsection{Post-launch Requirements}

The Inukshuk project to create the Cybercartographic Atlas of Indigenous Perspectives and Knowledge, including the Treaties Module, had started only 11 months earlier, virtually from ground zero in terms of geonarrative focus. With the cybercartographic framework, my background knowledge and my thesis vision, the Treaties Module was ready to be expanded into an Atlas. But first, there were a few things to wrap up, and funding that would need to be applied for in order to support the proposed atlas project.

During the period following the launch of the Atlas, work along the conceptual dimension occurred with the writing of a paper on the project (Caquard and others, 2009) and two conference presentations concerning the Treaties Module. Several months after the launch of the Atlas, a decision was made to apply for SSHRC funding to support the expansion of the Treaties Module into an Atlas. The preparation for the paper, the conference presentations and proposal writing included research into critical cartography and provided an opportunity both to situate the atlas project in ongoing conceptual dialogues, and to draw on insights from those dialogues in the atlas project.

First, I prepared a presentation for the Canadian Association of Geographers Annual General Meeting in Quebec between May 20 and May 24, 2008 to fulfill one of the final project requirements. Preparing the presentation allowed me to work out some ideas, and provided me with my first opportunity to explicitly link the atlas project vision with the concept of "reconciliation". The day before my presentation, a good friend of mine told me about the upcoming federal government reconciliation initiatives with respect to Residential Schools. 
This made me think about the need for reconciliation in other areas as well, in particular treaty relationships. After I returned to Ottawa, I began reviewing the literature on "reconciliation.

The idea for reconciliation trail map developed through my work to prepare for a presentation at a national conference, which was a funding requirement under the Inukshuk Wireless grant. This in turn led to further conceptual development, which would lead me months later in my thesis proposal to identify the proposed Atlas as a reconciliation tool through its ability to include multiple perspectives across multiple dimensions in a way that presented a "spatialized" view of history, something that the reconciliation literature was calling for as a necessary ingredient in reconciliation processes " (Bhandar, 2004 and Dwyer, 1999).

In addition to the presentations, I participated as a co-author in the writing of "A 'Living' Cybercartographic Atlas of Indigenous Perspectives and Knowledge of the Great Lakes Region" (Caquard et al., 2009). While I had engaged with a good bit of historical work during the design and development of the Treaties Module, and had learned about archival research and survey practices, this was my first opportunity since April 2007 to begin engaging with the critical cartography literature. This was also the first time I had participated in the writing of a peer-reviewed article, and the peer review process turned out to be very beneficial. In addition to a number of very useful comments, I was given some invaluable references to authors in the critical cartographic tradition such as Margaret Wickens Pearce (2008) and David Turnbull (2007). After 11 months of cybercartographic atlas-making practice, I was happy to see many 
links between what critical cartographers were talking about and what I had been doing.

\subsection{Travels, Work at the Lab and Funding Proposal Preparation: Spring - Summer 2008}

Following the Atlas launch, I accompanied Gimaah Wilmer to several meetings, including the meetings where the motion to create the Lake Huron Treaty Commission was being "moved" (and adopted). Altogether over his life, Wilmer had been Gimaah of the Magnetawan people for 18 years, and he had many stories to share, not only about his life as Gimaah. Wilmer and I discussed plans for different map projects within the Atlas context, including an Elderyouth wild rice re-harvesting project; a map of river travel based on Wilmer's experiences; and a 'working together map' reflecting Wilmer's experiences after leaving residential school at 15 and joining the non-Native workforce right away. He told me many stories of his various work assignments, from working on the rails either in the scorching sun or else in the freezing cold; working in a paint factory; building stone fireplaces for cottage owners on the different Lake Huron islands, and, later, installing septic systems for these same people.

Although the project to expand the Treaties Module was still the focus of my thesis work, the Inukshuk grant had expired. Fraser Taylor assigned me to participate in the Arctic Bay Atlas as a community liaison to aid in preparing the content to be uploaded to the Atlas. From an inter-project iterative processes perspective, working as a research assistant gave me the opportunity to learn about and work with the new software, which the Treaties Module would later be converted to in phase II. 
In addition to my practical work as a research assistant, I participated with Fraser Taylor and Barbara George in the drafting of a Social Sciences and Humanities Research Council Standard Research Grant proposal. Fraser had offered to support my thesis research to expand the Treaties Module into an Atlas, which would require funds for technical support and travel to the communities and conferences, not to mention the graduate research assistant funding that I would require in order to subsist.

Preparing the "program of research" provided me with an excellent opportunity to become more familiar with the critical cartographic literature, and to frame the proposed project within the grant specifications and guidelines. Similar to the way Fraser Taylor has described the evolution of cybercartography, which began mostly on the basis of intuition and practical work, and involves the holistic relationship between theory and practice, my first 11 months working on the Treaties Module emphasized practice and content-related research.

After the Treaties Module was launched within the Atlas of Indigenous Perspectives and Knowledge, it was time to reflect on the conceptual links and implications of the work we had completed. As with most proposal-writing processes, this process was intensive, especially since my mother, whose health had been steadily declining over the summer, had been recently diagnosed with cancer - luckily a very treatable form. I continued to work on the proposal - often at the end of a hospital bed - while my especially brother, Jason, my son Jesse, my sister, Angela and my three aunts, Nancy, Debbie and Susan, all provided support at this time. As my thesis supervisor, Fraser was also very supportive and encouraged me to continue working on the proposal, which I did. 
Once the grant application had been submitted, I needed to focus on fulfilling my academic program requirements: the comprehensive exam and the thesis proposal, which had been delayed by departmental standards due to my concentrated efforts working on the Treaties Module. For the next seven months, I would continue to concentrate on conceptual aspects of the atlas project. My mother's health improved steadily during these months, and in March 2008, Fraser contacted me and told me that the funding application had been successful. Soon, we could begin the work related to expanding the Treaties Module into an atlas. 


\section{Navigating the Narrative Logic of the Map of Maps}

This chapter reflects on some of the editing decisions that were made to create the various maps, and includes some discussion of the iterative processes that went into their making, their significance and their relationship to some of the other maps in the Atlas. These decisions reflect the narrative logic that has emerged in the atlas-making process. The Map of Maps is a good map to use as a guide that begins to reveal the narrative logic that is emerging in the Atlas. James Akerman (1991) has referred to an Atlas as a "map of maps". This chapter will examine some of Akerman's ideas in relation to the Map of Maps. The story of the Map of Maps involves the telling of other map stories, illustrating the performative and dynamic relationships between the Atlas maps, which are always engaged in iterative processes of change and transformation.

The maps in the Atlas are actually related in a cascade of reflexivity. The Map of Map frames the Travels in the Making of the Atlas Map, which frames the other maps in the Atlas. 


\subsection{The Map of Maps}

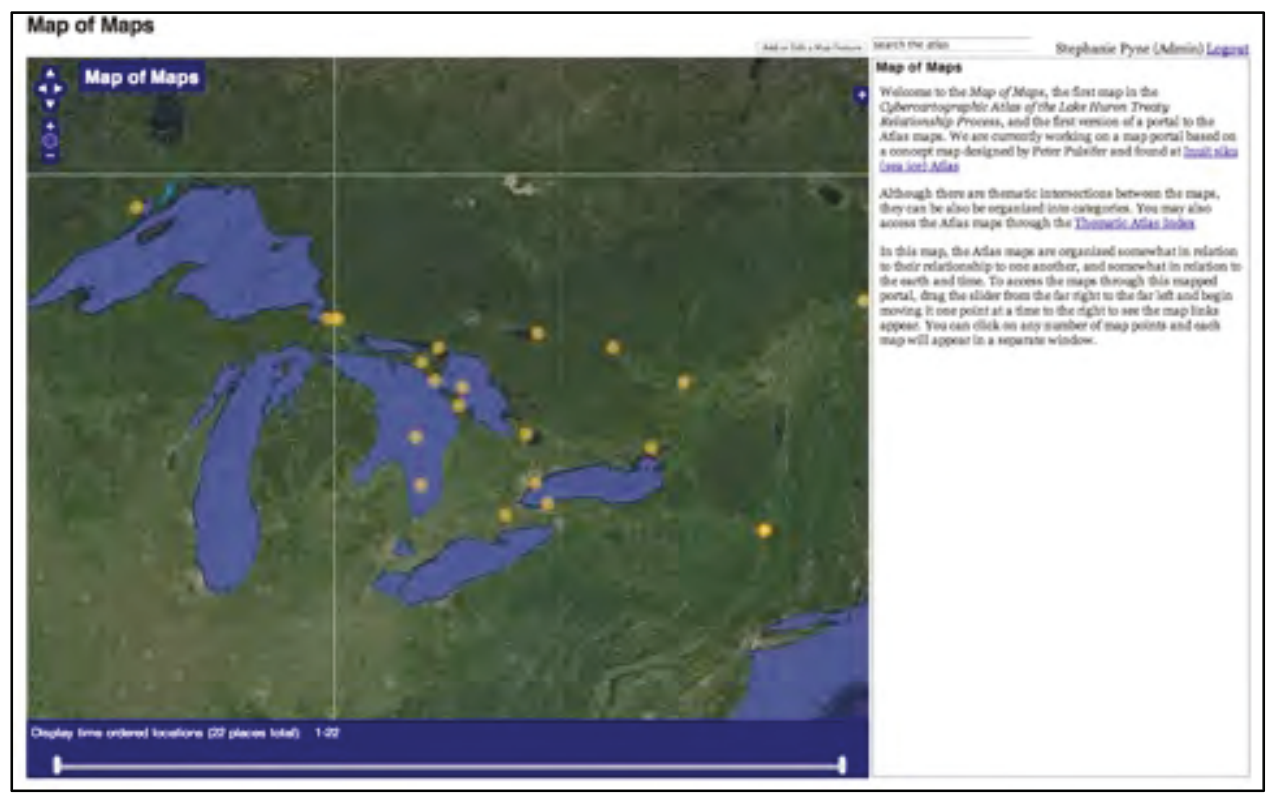

Figure 9.1. Screenshot of the Map of Maps showing points for all of the maps in the Lake Huron Treaty Atlas.

The Map of Maps was created prior to the launch of the Lake Huron Treaty Atlas at the end of Phase II in May 2012. I wanted a map portal that was not strictly thematic as the atlas Table of Contents, which is based on the Atlas working index. This index had developed over time through multiple iterations with the addition of various maps, and the occasional repurposing of maps.

The Map of Maps is a good example of an Atlas map that was repurposed. It was once titled "The Artists Map", a preliminary attempt to present contemporary Anishinaabe artists who I had met or whose work I had come across. Noticing a common decolonizing thread to all of their work and an almost equally common concern with history, my first foray into mapping this community of practice was to map each artist's general location and provide links to some of their work. Although I was aware that none of the maps in the Atlas would be launched as complete versions, I was not entirely satisfied with 
this map. Also before I included this map in the Atlas, I wanted to inform the people I was mapping of my intentions and discuss the map with them. After a telephone conversation with artist Bonnie Devine, from Serpent River First Nation, one of the artists I intended to include in the Artists Map, I decided not to publish the Artists Map, but to take more time. This decision followed my learning from Bonnie about a national database, which - although it was organized alphabetically and not geographically - performed the same basic function as the Artists Map. Bonnie was not entirely satisfied with the database and was hesitant to participate in similar projects. At the same time she expressed an interest in collaborating on some new form of map-art project that was consistent with her artistic interests. I told her about the discussions I had begun with Nadia Myre along those lines. These discussions have since begin to sprout in phase III into more extensive collaborations around designing and developing at least one or a series of cybercartographic geonarratives to express the artworks associated with Nadia's five-year collaborative Scar Project.26 Being so close to the Atlas launch, I told Bonnie I was going to hold off on the Artists Map for now and that we could possibly resume our discussions of possible collaborative mapping ideas in the future. And so, the Map of Maps was born. The decision to take the turtle's pace with respect to the Artists Map had resulted in a transformation to a new map, the "Map of Maps", which would allow the geonarrative logic of the relationship between the Atlas maps to emerge.

\footnotetext{
26 See the following link for more information about the Scar Project: http:/ / www.nadiamyre.com/Nadia_Myre/portfolio/Pages/The_Scar_Project.html
} 


\subsection{The Travels in the Making of the Atlas Map}

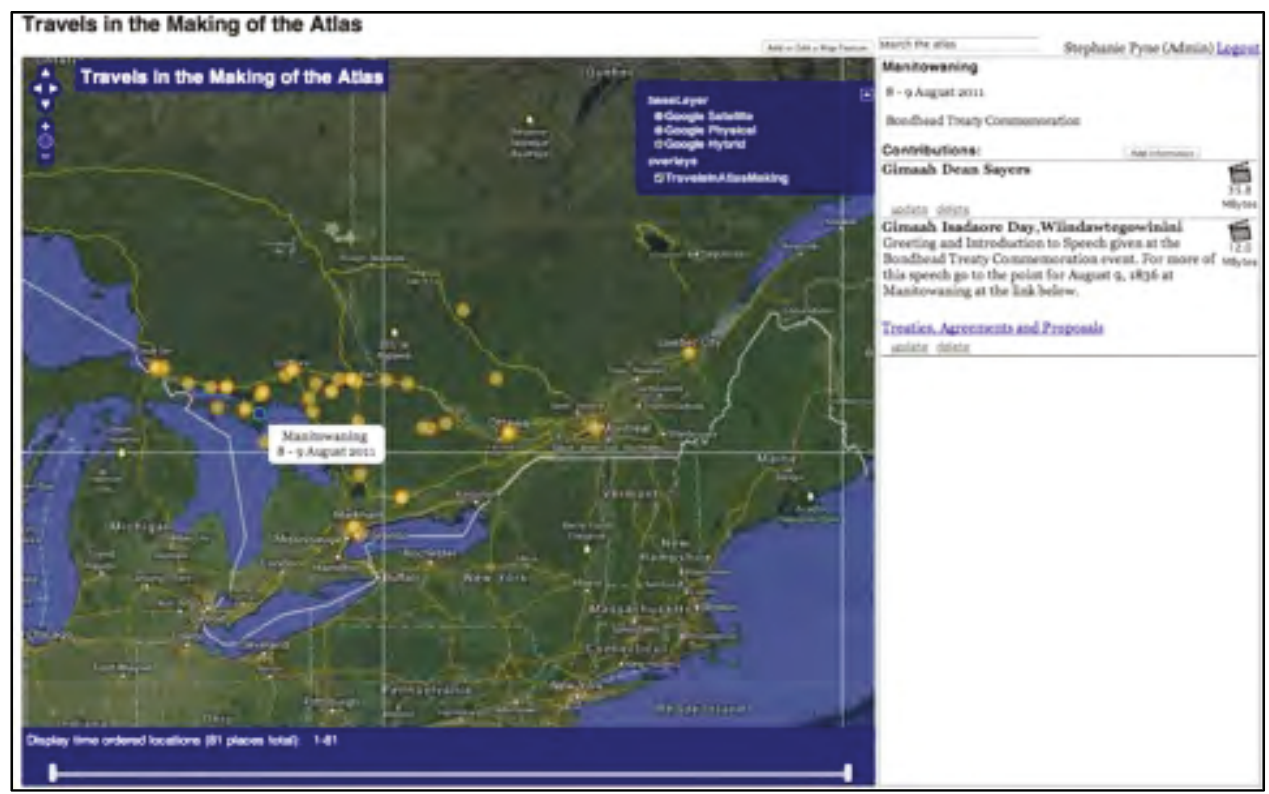

Figure 9.2. Screenshot of Travels in the Making of the Atlas Map showing some of the stops in my atlas-making travels; with the point for Manitowaning highlighted and the associated media and media descriptions visible along with the pop-up window displaying different map viewing options.

The idea for the Travels in the Making of the Atlas Map emerged out of the work to create the Treaties Module and was included in my thesis proposal to expand the Treaties Module into an atlas: "Other interactive map ideas include a series of interactive "reflexivity maps", which are envisioned to be actor networkstyle maps that trace the evolution of the making of the module through the development of the growing community of collaborative investigative relationships" (Pyne, 2009).

Key challenges in terms of map design include finding ways to link to other stories. These included decisions about how much and which media to include for each stop, and styling considerations

Following along in the Travels Map opens people up to interacting with the other Atlas maps by mirroring the processes through which these maps 
emerged. The reflexive journey approach to navigating through The Travels Map is the essence of its narrative logic. As the map that essentially documents some of the iterative processes involved in the making of the Atlas maps - with the potential to map them all - the Travels Map is placed first along the time dimension in the Map of Maps. The Travels Map functions as a narrative tool by tracking my movements across space and over time, and events have been sketched in the map that are relevant to understanding the development of the other maps in the Atlas.

As I was constructing the map, I wanted to place at least one piece of media with each point on the map. In the process of uploading video clips of Gimaah Isadore Day talking at the Bondhead Treaty Commemorative Event (Manitowaning, August 8, 2011), I came up with the mapping solution to include the clip with his introductory remarks in the Travels Map, and then provide a link to the Treaties, Agreements and Proposals Map (discussed below) where the remaining video clips related to the event would be uploaded.

That editing decision had great significance as far as atlas narrative logic goes, for I had found a way to link maps and enable people to navigate between the maps in order to follow a story. I sent Gimaah Day a message telling him about how I planned to present his video clips and he approved. This decision and the mapping strategy that it yielded can be considered as a template, a design pattern that I could apply in other mapping situations. This also illustrates the emergent nature of the mapping processes I have been engaged in, and the importance of engaging in mapping practices in the process of discovery.

The Travels Map itself already contains an explanation for why the first map is placed on highway just outside of Ottawa: "to reflect the importance of 
my first trip to the Lake Huron Treaty region. Although I had made two trips in 2007, this was the first trip where I was able to meet people from the treaty region, show them a very preliminary working version of the Treaties Module and discuss the vision to expand the module into an atlas. The Travels Map documents this and other trips that I made between 2007 and 2011, and it notes the significance of February 14 as the date of departure.

\subsection{The Treaties Module}

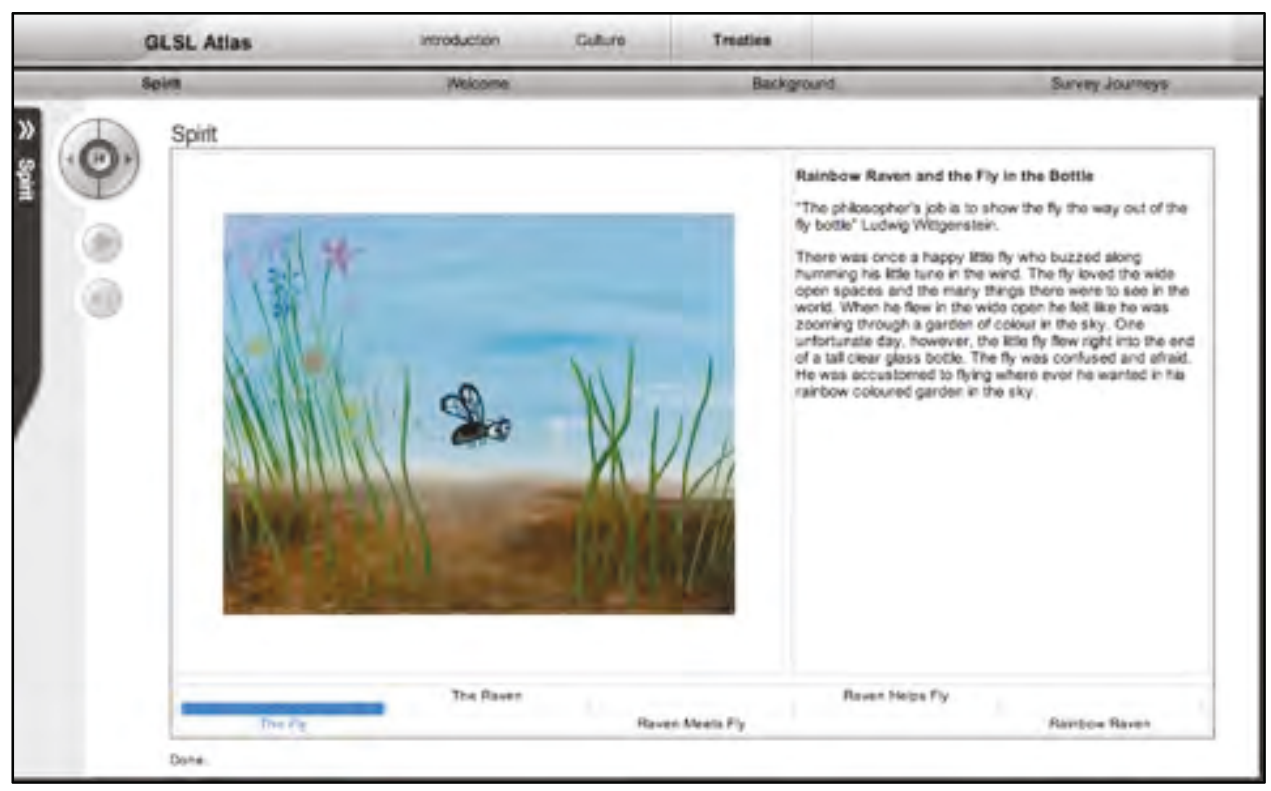

Figure 9.3. Screenshot showing first pane of Treaties Module of the Atlas of Indigenous Perspectives and Knowledge.

Although the processes that went into the making of the Treaties Module were described in chapter 8 , it is worthwhile noting that I placed the Treaties Module as the next map in the Map of Maps to draw attention to the first phase of Atlas work and to provide Atlas users with links to the Spirit, Welcome and Background sections of the Treaties Module, which were not entirely reproduced in the Atlas. The summary for this point provided in the Map of Maps reflects this: "The Treaties Module was included in the launch of the 
Cybercartographic Atlas of Indigenous Perspectives and Knowledge (Great Lakes-St. Lawrence region), which has a different look and feel than this Atlas because it was built in the Nunaliit I framework".

The Spirit sub-module represents in a story form the spirit and intent of the Treaties Module: to put together the various parts of the Lake Huron Treaty process story in a way that enhances awareness of the ethical aspects of its many interrelated dimensions and can contribute to knowledge of how to engage in healthy and fair treaty relationships today. The Welcome sub-module includes guiding concepts for a critical learning approach. For example, it draws attention to the existence of different perspectives, identifies four important criteria of "good" treaty relationships (fairness, balance, care, and mutual understanding), and invites viewers to assess the treaty process according to these criteria. The Background sub-module involves a time-lined account of some relevant historical background to the Lake Huron Treaty survey journey process.

The Survey Journeys Maps are based on the survey diary of J.S. Dennis (1851), which was transcribed and mapped out with the aid of diary descriptions, survey plans, current and historical maps and satellite imagery. In addition, each overnight stop in his journey has been overlain on one of two significant historical background maps. The "Enroute" and "Return" maps show a series of camp stops set against a standard settlement map, which tells a story in itself and illustrates the potential for maps in general as effective narrative vehicles; while the "In the Field" camp stops are set against a map drawn during the 1849 pretreaty investigative process by Commissioner Alexander Vidal (1849) depicting his impressions of Anishinaabe territories in the Lake Huron region, along with mining lots within the officially demarcated "Indian Territories", which had been 
applied for by prospectors. The diary entries associated with the stops are included to the right of the map, together with an audio clip of a voice representing Dennis. The diary entries are transcribed from Dennis's original handwritten report.

Iterative processes occurred between the content, technology and concept dimensions in the further development of survey journeys maps and the extension of the Treaties Module (produced in Phase I) with one three part map into the Lake Huron Treaty Atlas in phase II comprised of over 30 maps.

\subsection{The Survey Journeys Maps}

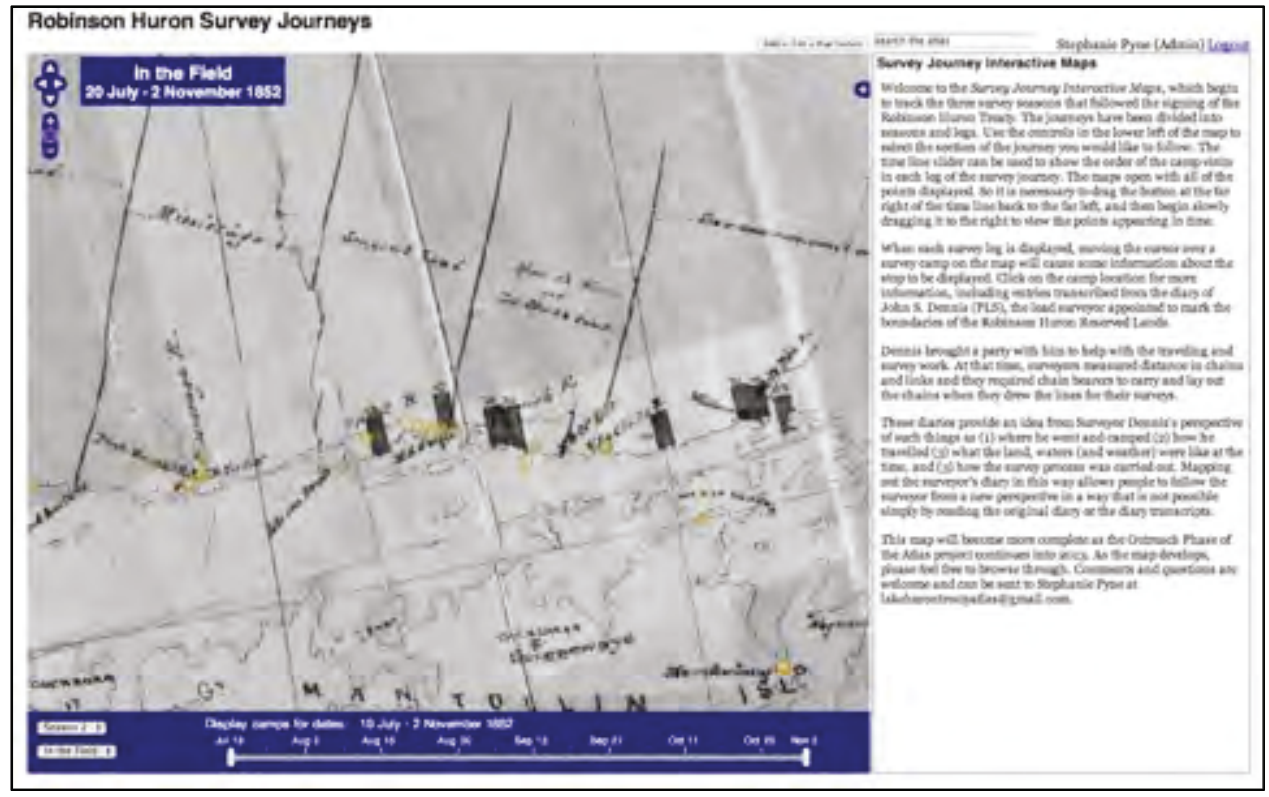

Figure 9.4. Screenshot showing Season Two, In the Field of the Survey Journeys Maps set against Vidal-Anderson Commission, 1849 proposed reserves sketch map and mining lots (in black) surveyed prior to the Robinson Huron Treaty signing.

As stated in the entry for this map in the Map of Maps,

The survey journeys maps were the first maps to appear in the Lake Huron Treaty Atlas. They were transferred or migrated from the Treaties Module. The point for the map is placed along the coast of the Bruce Peninsula in the Georgian Bay. The surveyor's travels by steamship to and from the Treaty region included this point. 
Placing the point in the water draws attention to a centrality of water in the survey story, since all travel involved both the water and Anishinaabe navigational knowledge.

This passage reflects a geonarrative logic that de-emphasizes the importance of precise location and instead highlights the centrality and shared nature of water.

The transition from phase I to phase II of the project involved converting the Treaties Module from the Nunaliit I to the Nunaliit II framework, which offered more possibilities for developing fully interactive wiki-style geonarratives. During the conversion process, the second season of the survey journeys were also mapped, thereby expanding the map's coverage. While template maps for the third season were also created, the work was not yet done to map the camp spots indicated by the third season surveyor, J.W. Bridgland, who used a slightly different reporting method than Dennis had used for the first two survey seasons. In addition, the third season involved the survey of only one reserve under the Lake Huron Treaty with the remaining surveys being completed for reserves under the Lake Superior Treaty, which was signed the day before the Lake Huron Treaty. To this day, there remain questions about the processes involved in the signing of these two sister treaties. Prior to the completion of phase II and the launch of the Atlas in May 2012, atlas contributor Joel Chamberlain went through the Bridgland diary and mapped the approximate camp locations from the spot I had left off at in the 'In the Field' Map during my initial attempts at this mapping. However, my early mappings of the 'Enroute' Map for this season had yielded insights into changes associated with the wave of interest in railroad development. While Dennis had taken the stagecoach to Lake Simcoe from Toronto only the year before, his successor, 
Bridgland, was able to dispense with the stagecoach at present-day Vaughan (Concord on the map), at which point he travelled by train to Lake Simcoe. Despite the progress made so far with sketch mapping Bridgland's survey journey, the geonarrative editing challenge still remains: How to map the third season in one map when it reflects the processes of two treaties. In addition, the title of the map, "Robinson Huron Survey Journeys" does not accurately reflect the content of the maps for season three, emphasizing the need for a mapping solution. This need is reflective of the current and past situations with regard to the ambiguities that exist in treaty history and interpretation, ambiguities that have contributed to the inability of these treaties to be adequately implemented. Part of my role as geonarrative and content editor in phase III of this project involves finding the geonarrative solution to this mapping problem. It is possible that in finding this solution, a contribution could be made at the policy level in terms of treaty interpretation.

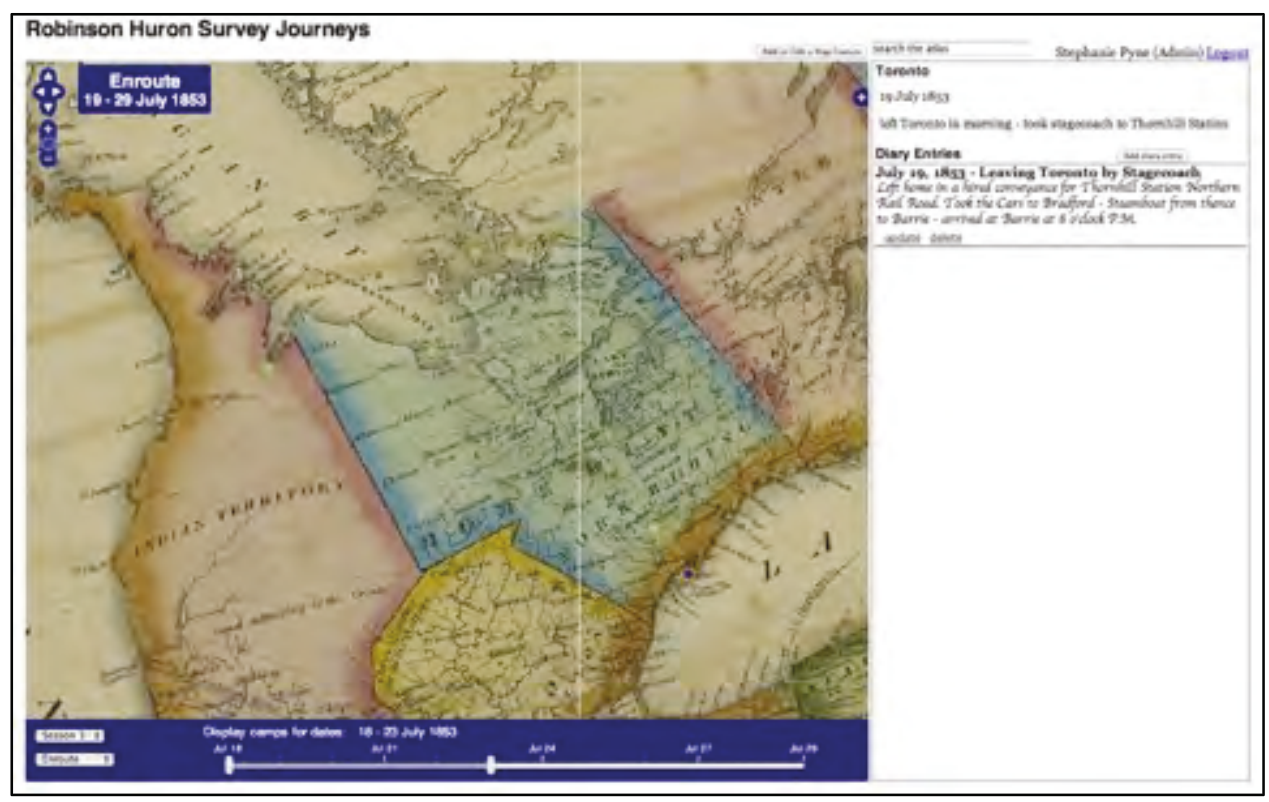

Figure 9.5. Screenshot showing the Season One, Enroute Map with Bridgland's travel stops set against Commercial Map of Canada, 1834. 
On May 27, 2009, the first version of the Lake Huron Treaty Atlas was uploaded to the server; although at the time it was referred to as the Cybercartographic Atlas of the Lake Huron Treaty Relationship Process, which is more reflective of the Atlas' contents but also long and unwieldy for repetitive use. The Atlas was comprised initially of six maps, three for season one and three for season two of the Lake Huron Treaty survey journeys. The first iterative transformation to occur in Phase II was along the technology dimension with the decision to convert the Treaties Module content to the Nunaliit II open source Atlas framework. Around this time and following, the technological and content dimensions began to develop iteratively as the technical and content specialists began to have regular meetings concerning the form and functions of the maps envisaged for the Atlas. It was decided that the initial priority would be getting these maps up and running in the Nunaliit II framework; then thinking about how to integrate the content of the Spirit, Welcome and Background submodules, if to integrate them at all. Once the survey journeys maps were working properly, it was thought that attention would turn to the creation of a map template for some of the additional maps planned for the Atlas.

The Survey Journeys maps ended up developing in a way we had not expected. Although all of the content for the season two Survey Journeys Maps had been entered into the system, the maps for this season were not published in the Treaties Module that was launched at the end of phase I. It was not until the conversion of the Survey Journeys maps to the Nunaliit II software framework, with its improved usability, that I was able to review the Season Two maps. Through this interaction I was reminded that at a certain point - contrary to his official Instructions, the principal surveyor, J.S. Dennis, delegated some of his 
instructed duties to a junior surveyor, Arthur Bristow. For several weeks, Dennis was in one place reporting on his own progress in that location, but at the same time appending a summary of the junior surveyor's progress in an entirely separate location (Dennis 1853). And the decision was born to create a separate map trail for the second surveyor featuring his reports appearing in the vicinity of where he actually was. This addition to one of the Survey Journeys maps was an important innovation and the result of iterative processes along several dimensions: content, conceptual and technological (Brauen et al., 2011).

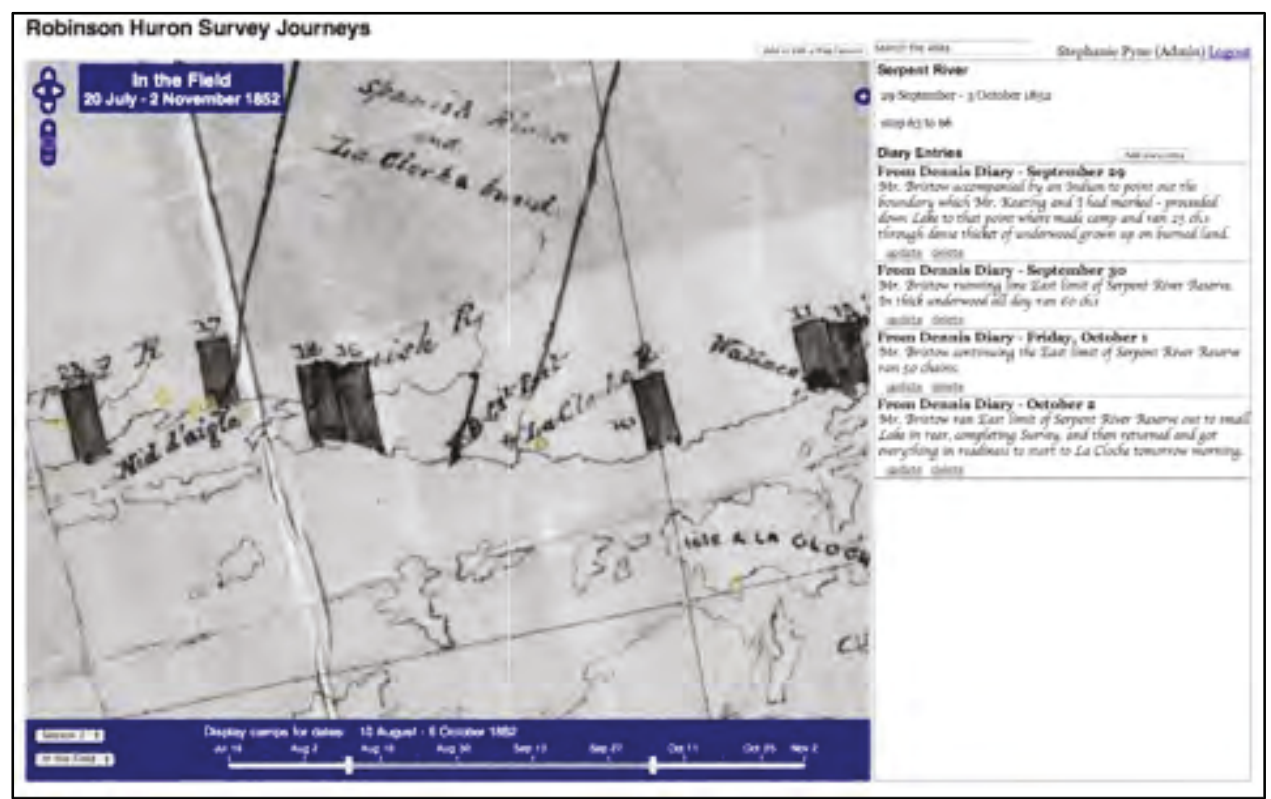

Figure 9.6. Screenshot showing the 'Season Two', 'In the Field' Map with pale green dots representing Bristow's camp stops and yellow dots representing Dennis' camp stops.

The work to create a Survey Journeys map with two synchronous trails provided a prototype for mapping multiple trails that can be used in other maps. In addition, the Survey Journeys maps provided the basis for the design of the more generic "biography map" template, which was subsequently used for most of the later maps added to the Atlas. Closer to the end of phase II, continued 
discussions about the design of a critical map layer for the Survey Journeys and other maps in the Atlas led to the decision to the convert the Atlas once more, this time to a Couch DB atlas software framework. The idea of creating a critical layer that can be viewed in conjunction with the original comments layer (initially proposed in phase I) remained strong throughout phase II as an agenda item for development in phase III.

Once the issues related to mapping the trail of the second surveyor had been sorted out and the new map with two trails had been released, attention could turn to the design of the new maps planned for the Atlas. The first idea was to use the data compiled for the first version of the map based on Marlatt (2004) to create the Marlatt Commentaries Map, a map that could also include some of the content from the Background sub-module of the Treaties Module, in addition to new background content.

\subsection{The Nations not Surveyed between 1851 and 1853 Map}

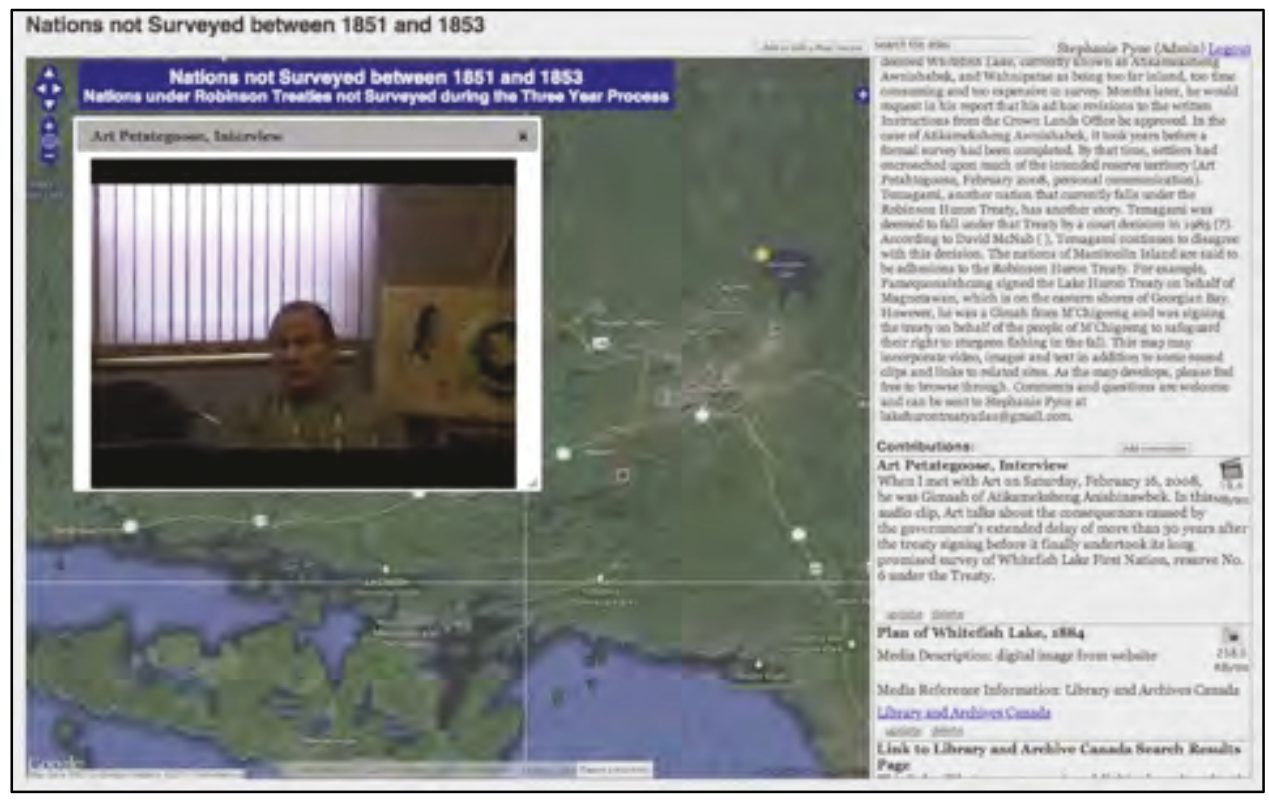


Figure 9.7. Screenshot showing the point for Atikameksheng Anishnawbek highlighted in blue and the video clip of Gimaah Art Petahtegoose talking about the consequences for his community of not surveying the reserve.

As stated in the Map of Maps:

The point for this map is close to the point for the Survey Journeys maps to draw attention to the fact that the Nations on this map were not surveyed during the official post-Treaty signing survey process. This map is at a very early stage of development. One highlight is a video of Art Petahtegoose talking about how long his community, Atikameksheng Anishnawbek, had to wait before an official survey was completed.

Although the Nations not Surveyed Map was not created until just prior to the launch, the nations that were not surveyed could not be included in the Survey Journeys Maps, since they were never visited by the Robinson Huron Treaty surveyor. This situation necessitated the creation of the Nations not Surveyed Map, which - although it only has two points - provides a space where a lack of treaty implementation history can be told. This map contains a video clip of Gimaah Art Petahtegoose discussing the many attempts that his ancestors made to get the surveyors to do a survey of the reserve pursuant to an agreement made at the treaty signing; and the subsequent unauthorized settlement of Atikameksheng Anishnawbek territory by newcomers. 


\subsection{The Marlatt Commentaries Map}

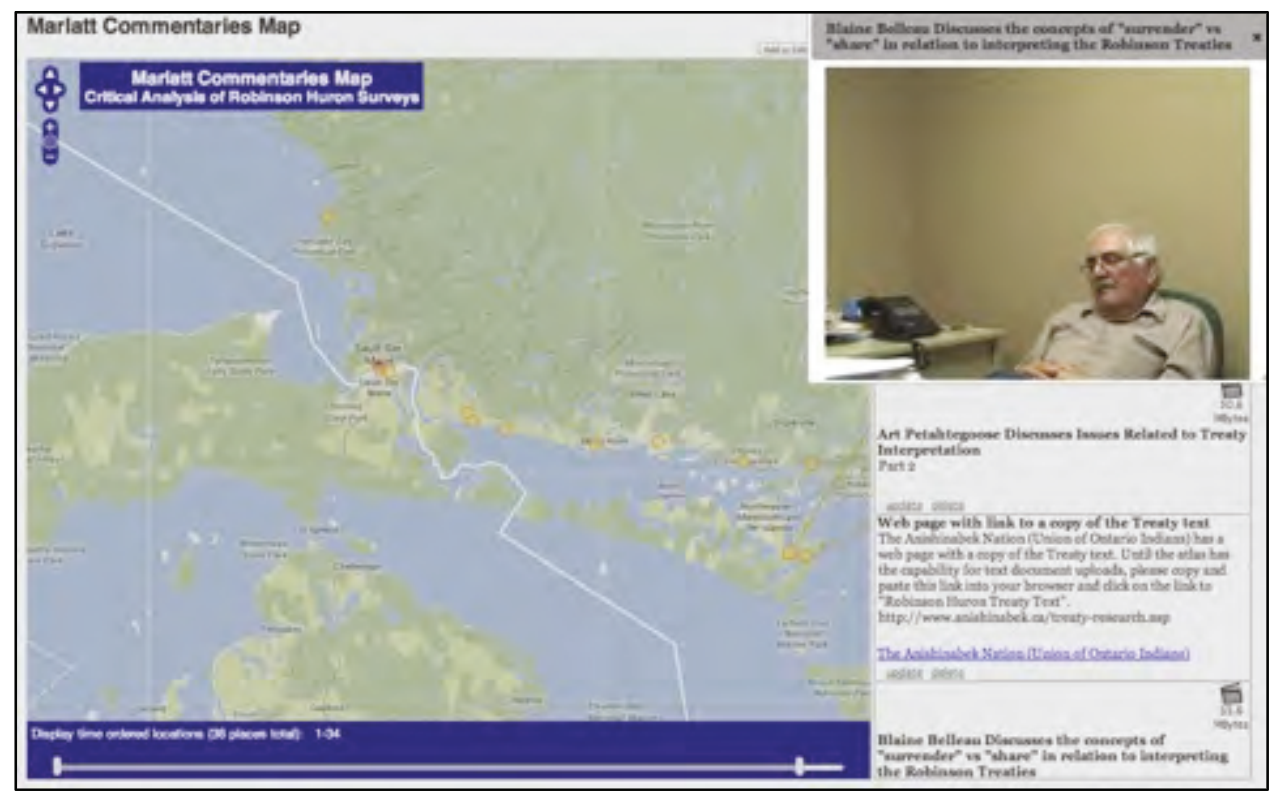

Figure 9.8. Screenshot showing point for the Robinson Huron Treaty Signing Ceremony at Bawating (Sault Ste. Marie), September 9, 1850, with video clip of the late Blaine Belleau discussing the difference between "surrender" and "share".

The subheading "A Critical Introduction to the Survey Process" precedes its description in the Map of Maps:

This map was actually conceived of - and a basic prototype was created - before the Survey Journeys maps were created. It goes hand in hand with the Survey Journeys by providing a critical summary of the survey process conducted by Crown officials on behalf of the Anishinaabe signatories to the Robinson Huron Treaty. The point for the map is placed in North Bay because it is the region where Michael Marlatt, the author of the paper that inspired the map, is based.

As I said above, with the new survey journeys map platform available, I could begin to experiment with how to present the Marlatt chapter as a geonarrative. I decided fairly early to include some background to the survey process descriptions provided by Marlatt and included in the Background section 
of the Treaties Module; and in this way was able to find a way to integrate more of the Treaties Module content into the Atlas.

Some time in Phase II, I worked with Michael on editing the summaries of his chapter that I had prepared in Phase I. From time to time, as changes were made in the map, I contacted Michael and he reviewed the changes and gave his approval. When I travelled through the treaty region, I visited with Michael and showed him the new versions, explaining plans for the future. He provided me with season III material and critical commentaries and suggestions, and verified some of my geo-locations. The Marlatt Commentaries Map ties in with both the Survey Journeys Maps and the Governance Paper Trail Map, and was basis for deciding on the specifications for a generalized biography map template.

\subsection{The Treaties, Agreements and Proposals Map}

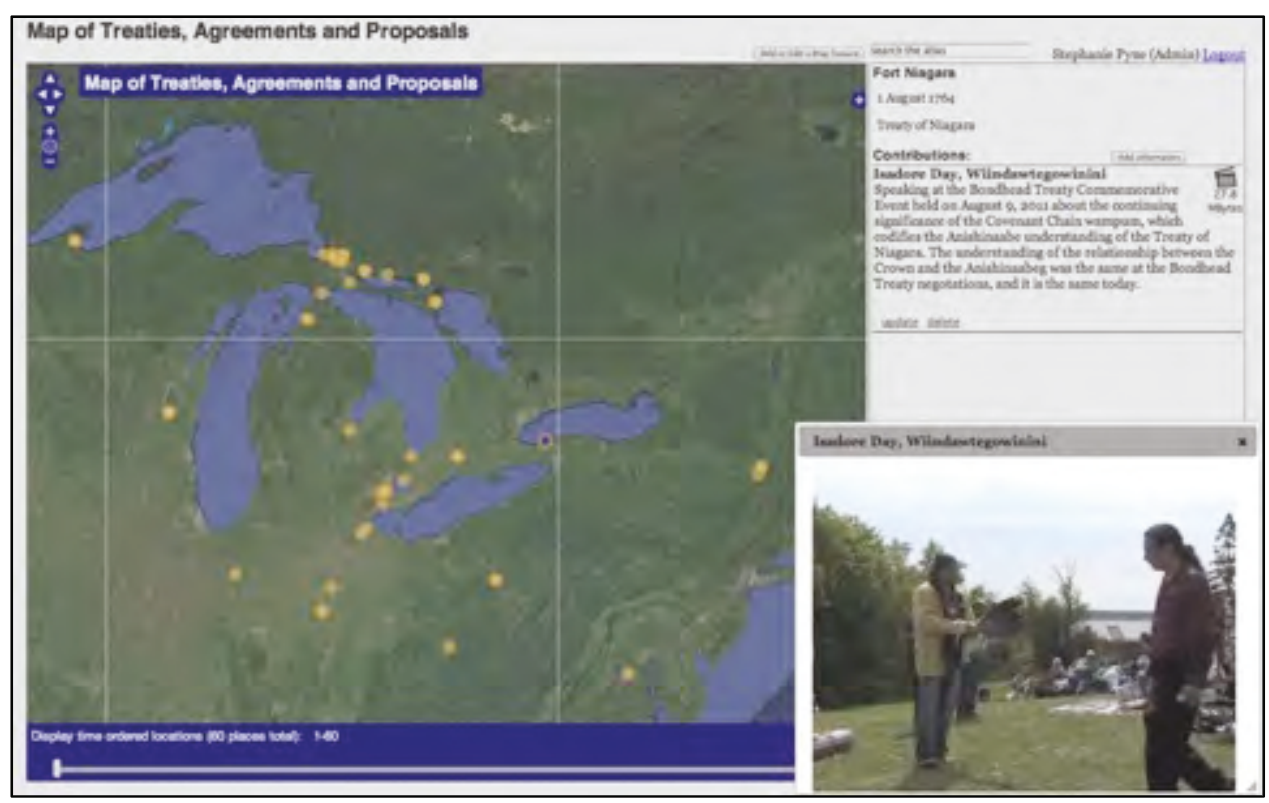

Figure 9.9. Screenshot highlighting the point in blue for the Treaty of Niagara, 1764, signing ceremony at Niagara Falls, with a video clip of Gimaah Isadore Day (Wiindawtegowinini) speaking about the significance of this treaty for future agreements between the Anishinaabek and the Crown, including the Bondhead Treaty; and with Alan Corbiere (mentioned above), walking in the foreground. 
The subheading "Expanding the Critical Perspective" is intended to draw attention to the broader context of treaties involving the Anishinaabek and either the British or American governments. As the description reads:

This map was not literally created on April 1, 2010. It is placed next in time to the Marlatt map to emphasize the thematic similarity between the maps. The map is placed at the point for Toronto because that was the seat of administrative governance in Canada West at the time.

This map was conceived fairly early on with the idea to present different scales of conceptual granularity among the different maps. It was also intended to provide geo-located references for various treaty signings, primarily in Anishinaabe country. Before the launch of the Atlas at the end of phase II, I mapped quite a few of the treaties referenced in the table at the back of Phil Belfy's Three Fires Confederacy (2011). This map is by no means complete, but a good start has been made, and each point on this map is an invitation to critique and comment.

\subsection{The Bagone-giizhig (Hole in the Day) Biography Map}

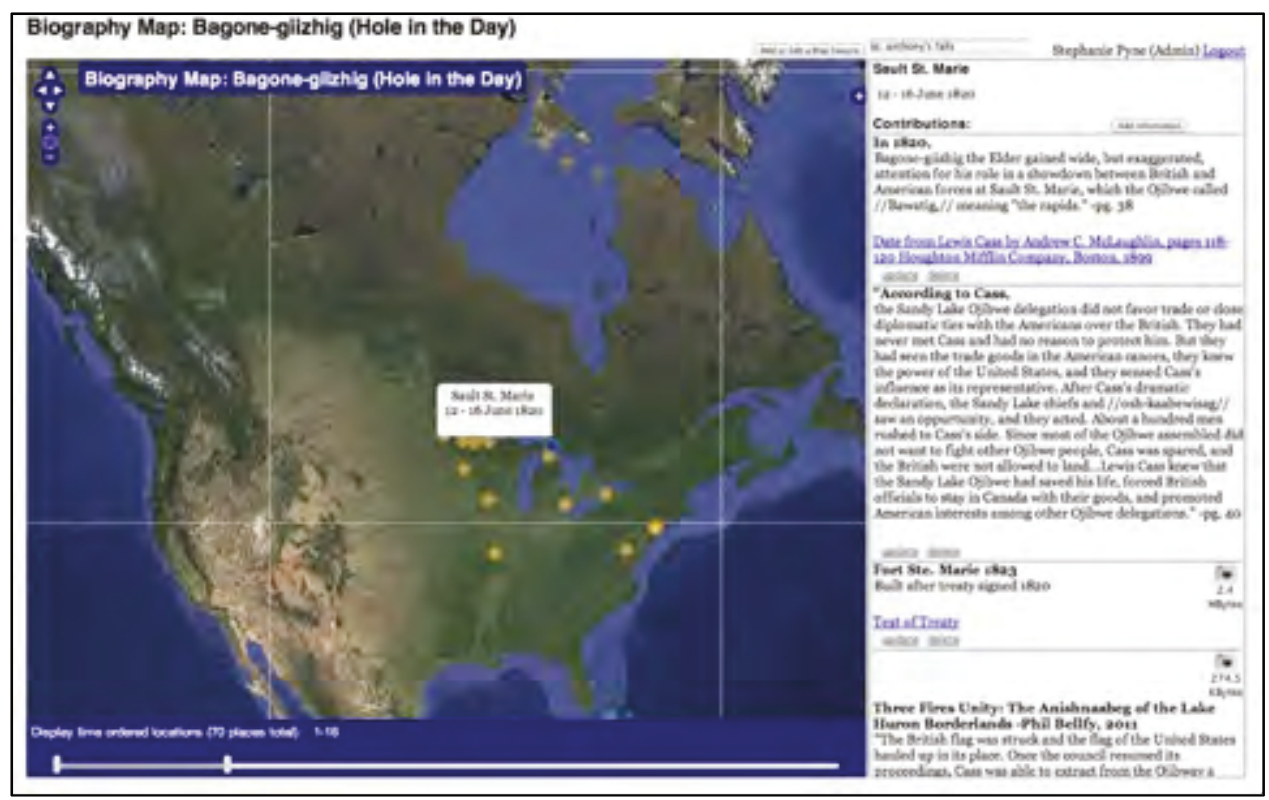


Figure 9.10. Screenshot showing the point for June 12-16, 1820 at Sault Ste. Marie, Michigan where Bagone-giizhig Sr. helped prevent the British from crossing onto the American side.

According to the description provided in the Map of Maps, "the idea for this map came after hearing a presentation by Dr. Anton Treuer, the author of the book this map is based on. The presentation was given in February 2011 at the Anishinaabewin Conference hosted by the Ojibwe Cultural Foundation. The point for this map is placed in Bimidji, Minnesota, where Treuer teaches".

Prior to the Atlas launch in May 2012, I wanted this map to be worked on a little more. Although much remains to be done with respect to enhancing this map, a lot was learned in the process of geo-transcribing portions of Anton Treuer's book, The Assassination of Hole in the Day (2011). The following introduction provided by Joel Chamberlain to the Bagone-Giizhig map provides an indication of the type of awareness that was gained through the mapping process by a volunteer to "geo-transcriber" who previously had little knowledge of the historical geographical context surrounding this story, let alone the story itself:

Without prior knowledge, when reading Anton Treuer's biographical novel The Assassination of Hole In The Day, one begins to understand the changing dynamics that affected the lives of Bagone-giizhig and the Anishinaabek. Attempting to "map" the story reveals multiple challenges in relating historical information from reliable sources. Discrepancies multiply exponentially as more historical sources are considered and compared. Locating points on the map involves referring to archived maps made by cartographers from different countries who made a multitude of expeditions, and who had varying degrees of acuity. Discrepancies include different names in many languages for landscapes and waterways that continually change naturally, seasonally and as a consequence of human development; and contrasting interpretations of events from personal accounts of individuals with varying backgrounds, prejudices and professions, and with questionable motives and limited perspectives. 
Bagone-giizhig was born into a society under pressure from European cultures looking to expand their 'dominion', searching for resources to fuel their wars against each other, and willing to sign Treaties they had no intention of honoring. This became obvious while researching documents needed to map the history of events and the roles that Bagone-giizhig and his son had in mitigating the effects on their people from a country born of and into war, with other Nations and themselves.

One of the most interesting things about Joel's approach to mapping the Bagone-giizhig story was his attention to geomorphological details and surrounding contextual information about the places he was identifying. One of the most important aspects of this mapping process was the manner in which the need to identify St. Anthony's Falls (the burial location for Gimaah Bagonegiizhig Sr. who was the father of the protagonist indicated in the book title) led to questions concerning the many changes over time that had occurred at St. Anthony's Falls. In the process of mapping out events associated with locations and times in the story, Joel was also tracking environmental and "development" history associated with these places. Following this model that had emerged for him during the mapping process, Joel turned a contextually critical eye to political, military and economic contextual material as well, and mapped some of his findings. As a result, this map currently does not have continuity, since the story entries are interspersed with context entries. Although the map may be difficult to follow, Joel's approach to geonarrative became a mapping challenge. This inspired Glenn Brauen to begin working on mapping solution: a multilayer map, with a context layer, which could be displayed either with or without the story layer. Further developing this multilayer map template remains on the agenda in phase III of the atlas project. 


\subsection{The J.W. Keating Biography Map}

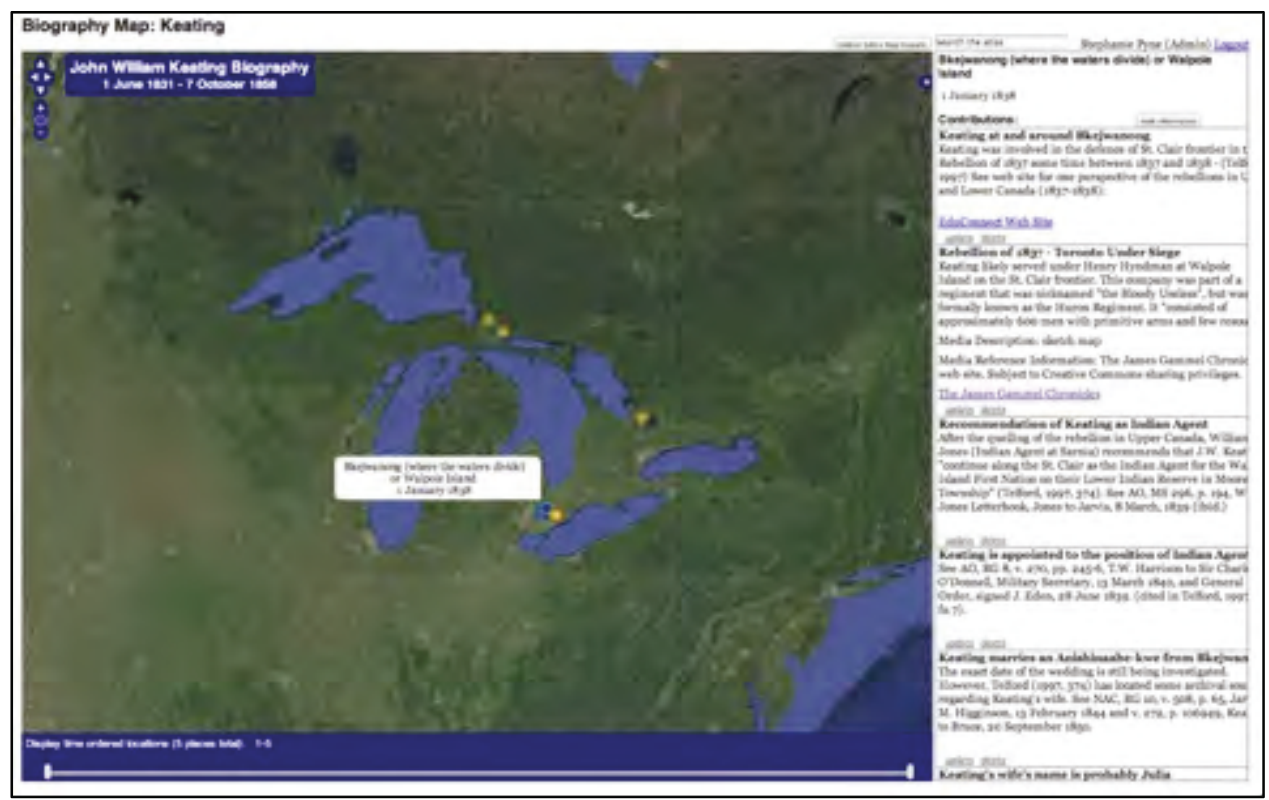

Figure 9.11. Screenshot highlighting point for Bkejwanong (where the waters divide) or Walpole Island, where Keating eventually became an Indian Agent.

The subheading "The People behind the paper" is intended to draw attention to the links between people, their characters and their institutional roles; with the idea of everything being related, the narrative logic of this map reflects the relational space concerns with power expressed by John Allen (2004). Relational space approaches allow for a penetrating analysis that examines previously unacknowledged dimensions and interpretations of these relationships. For example, John Allen's (2004) attention to the spaces of power "in between" officially delegated authorities has immediate analytical relevance to the interpretation of the "administrative" aspects of the Lake Huron Treaty relationship process, especially with respect to the effects of the relationship between certain "personalities" (as demonstrated in their actions and attitudes) and the powers they exercised under the authorities of their official positions. This map provides an example of attempting to become more familiar with the 
character of a key figure in the Robinson Huron Treaty signing and survey processes, and begins to shed light on the person and his life, beyond what is reflected in the Survey Journeys Maps. The iterative processes that have contributed to its making so far have occurred along each of the concept, technology, financial and content dimensions.

As the summary in the Map of Maps states:

J.W. Keating played a central role as interpreter and translator for both the Robinson Huron Treaty signing and Reserve Survey processes. At the same time he had interests in at least one mine in the area. A map tracking the man and his life helps to appreciate the links between a person's character and the way they carry out their official roles and duties. The point for this map is located in Oro County, the first place in Ontario where Keating may have lived with his stepfather, a retired military captain.

This map was initially based on the chapter "The Nefarious and FarRanging Interests of Indian Agent and Surveyor John William Keating, 1837-1869 (1997) " by Rhonda Telford. As Keating was not an overly famous individual, there was not much additional information readily available about him. However, I was able to locate some material through Internet research-based research as a start. On May 16, 2011, I emailed Rhonda Telford and asked whether she had any information about Keating's parents' names, the place he was born, or any places he had been prior to his involvement in the 1837 Rebellion. Over a series of 16 emails exchanges, which also involved Patricia Kennedy, we had exchanged a variety of information and on August 13, 2011, Patricia was able to locate Halifax as Keating's point of entry to Canada, along with some other valuable details.

This story provides an example of the way mapping processes can be collaborative and illustrates the fluidity with which contributions can be made. In 
addition, it highlights one type of emergent knowledge that can result through geonarrative mapping practices.

\subsection{The J.S. Dennis Biography Map}

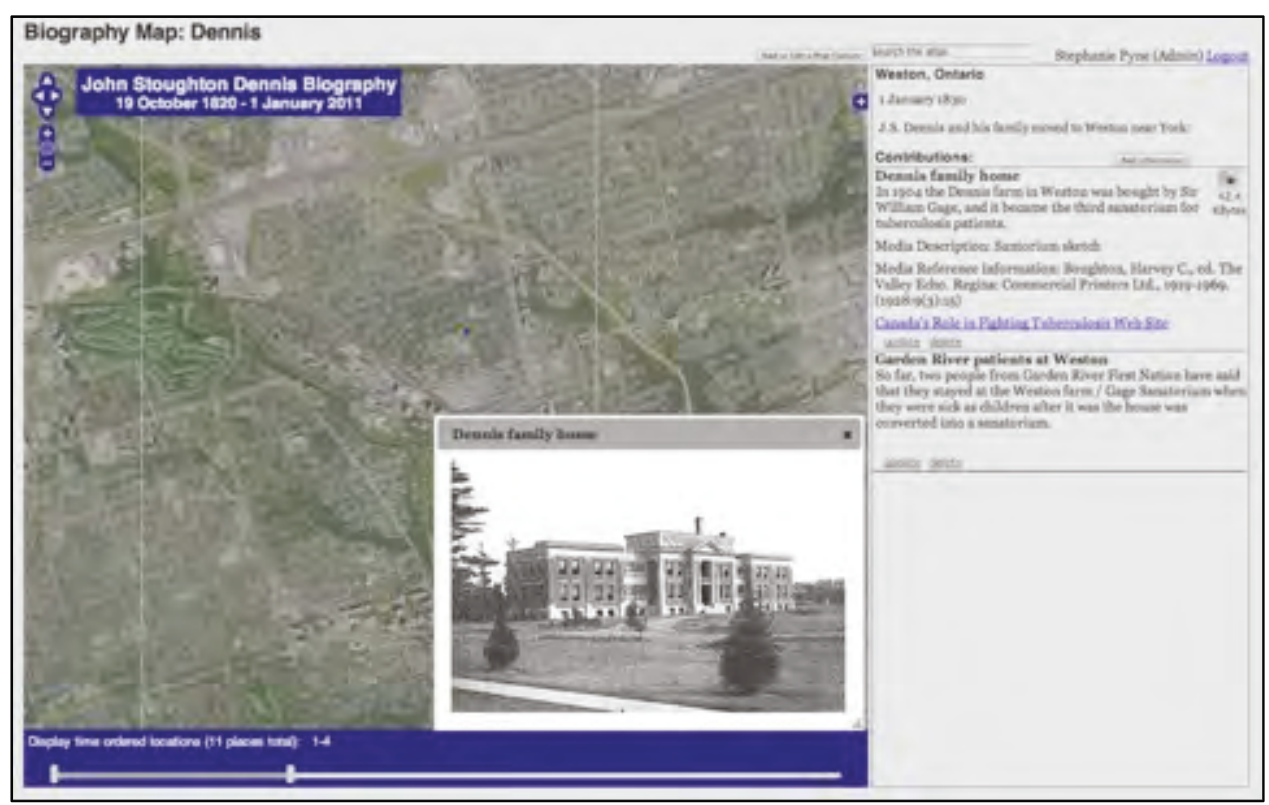

Figure 9.12. Screenshot highlighting the location of Dennis' childhood home in Weston (now part of Toronto). The home, which is featured in the pop-up image, was later sold and converted into a sanatorium.

This map is also in the 'People behind the paper' category and reflects a similar decolonizing motivation to read between the lines of power that is behind the J.W. Keating Map. As the summary for this map explains, "J.S. Dennis was the lead surveyor for the first two seasons of a three season survey process. His diaries, field notes and reserve survey plans complete the Robinson Huron Treaty by providing detailed specifications of extents of land that were significantly underspecified in the Treaty. The point for this map is in Kingston, the place where Dennis was born".

The Introductory text to the map itself provides a hint of the critical intentions of the map: 
Welcome to the Dennis Interactive Biography Map, which begins to share some of the biographical events of JS Dennis' life across time and space, and to shed some light on the character and relationships of a man whose travels are featured in the first two seasons of the survey journey maps. Dennis was responsible for the production of the official reserve survey plans, which he drew based on information from his diary and field notes. Considering his importance to the reserve surveying process, it would be helpful to know a little more about the man, his life and his character. Mapping other sources of knowledge about Dennis and his life provide the basis for this more in depth biographical map.

Engaging in the collaborative mapping of the life and journeys of J.S.

Dennis gave rise to the emergence of a variety of interesting knowledge. In Pyne and Taylor, 2012, we recount a particularly interesting example:

In the process of researching and mapping out the biography of J.S. Dennis ... we found information about the home he moved to with his parents in Weston (north of downtown Toronto) in 1830, when he was about 10 years old. In 1904, the home was sold to Sir William Gage, who transformed it into a sanatorium for tuberculosis patients. This information was relayed to Blaine Belleau, a member of Garden River First Nation and an atlas collaborator, while we were reviewing the working J.S. Dennis biography map during a meeting to work on Blaine's contributions to the Atlas. When Blaine saw the image of the home, he remarked in amazement, "I was there!" He then told us the story of how he had spent nine months in the sanatorium as a nine-year old when he was ill. In that moment, we all realized that Blaine had actually lived in the same house as J.S. Dennis, a man whose actions he had been studying for years in the context of lands research (97). 


\subsection{The History According to Blaine Belleau Map}

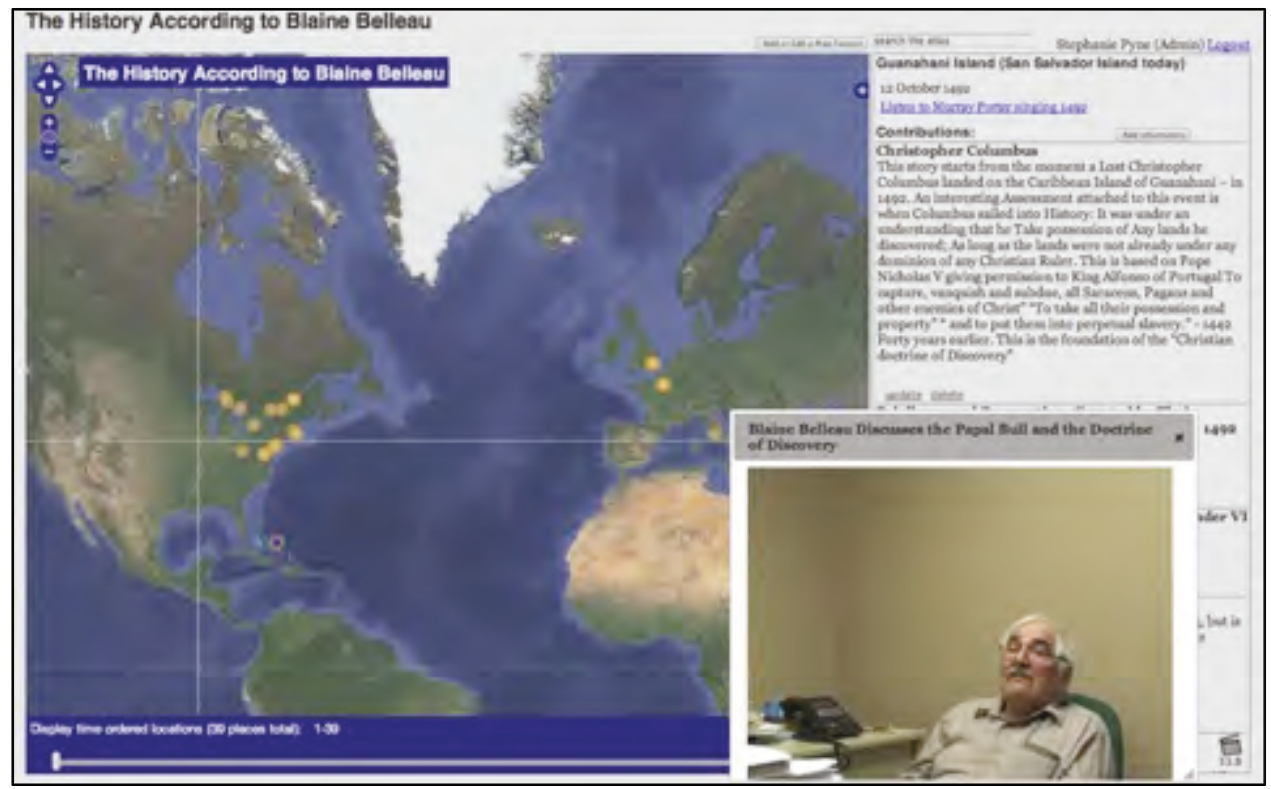

Figure 9.13. Screenshot showing the scope of the History According to Blaine Belleau Map, with a pop-up image of the late Blaine Belleau speaking about the mythical foundations of the 1493 Bull Inter Caetera by Pope Alexander VI and the Doctrine of Discovery.

The subheading "A Broader History" describes this map, which begins to sketch out a broad range of points in history that the late Blaine Belleau found to be significant and inter-related. The summary provided for this map in the Map of Maps introduces Blaine and the general nature of the map: "Blaine Belleau, a long time Garden River First Nation Band Councillor, was involved with the atlas project close to its beginning in the fall of 2007, and continued to contribute insights to the project until two weeks before his passing on July 23, 2013. The point for this map is located at Blaine's home community, Garden River. Thematically, this map is consistent with the historical focus of the Keating and Dennis biography maps; however it is broader in scope and includes Blaine's unique perspective". 
It took several years of reflecting and having continued meetings with Blaine, gathering video recordings of Blaine's teachings before it became clear how to map his contributions. In addition to the stories Blaine was providing, we discussed the mapping challenges relating to transforming his contribution into a geonarrative. Would his video-recorded discussions be dispersed across a series of Atlas maps, or would they constitute their own map?

Over the years of meeting with Blaine he circled around some topics more than once, which was fine with me. Blaine discussed a range of topics, many revolving around directly the treaty relationship theme. Then in meetings we had near the end of May 2010, Blaine shared an outline for a talk he planned to give at a conference entitled "Engaging Indigenous Communities: Resources, Rebellions, and Resurgence" to be held in August. I began to see how this outline constituted Blaine's geonarrative logic and proposed to him that we create the History According to Blaine Belleau Map to which we would upload video clips from his talks. This map would be based on his basic outline for the upcoming talk. Blaine agreed and the map was born. 


\subsection{The War of 1812 Map}

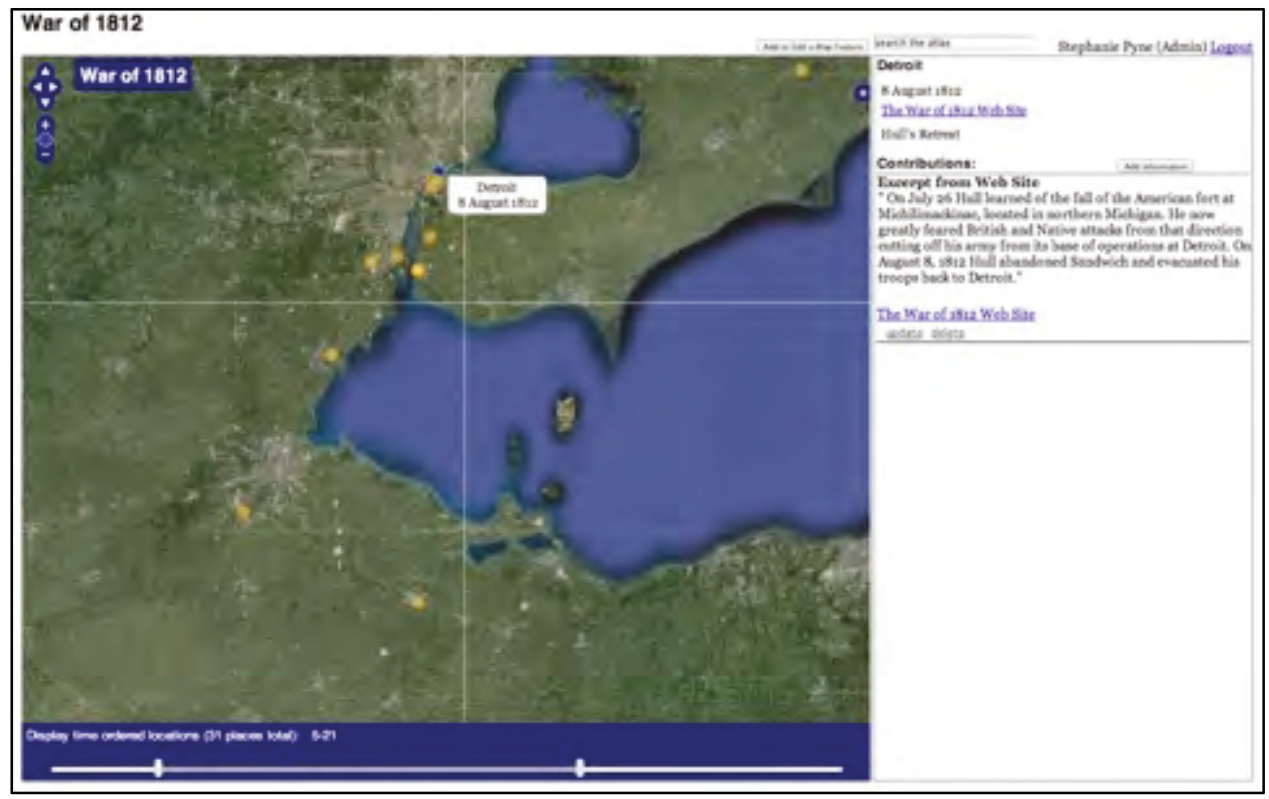

Figure 9.14. Screenshot showing sites of some key battles, skirmishes and events in the War of 1812.

The subheading "Military Allies" is intended to highlight Anishinaabek sovereignty in their dealings with British officials, and draws attention - in a subtle way - to the indispensible role played by the Anishinaabek in the War of 1812. The summary for this map provided in the Map of Maps hints a little further along these lines: "This map is located at the point for the Battle of Tippecanoe, a pivotal event in the commencement of the War of 1812. The military alliances between the British Crown and the Anishinaabe Nations from the Lake Huron proved beneficial to the British".

I began doing Internet research to construct this map at first, linking to relevant sites with interesting and useful information, and talked about the map with people in different communities. I kept in touch with Carol Nadjiwon, the historical researcher for Batchewana First Nation, to see if anything would be happening with their War of 1812 commemoration project and to share 
information I had found. I encouraged Carol to access the information via the map, which I was beginning to populate. I also sent Carol a link to the Atlas with instructions, and later visited with her to show her through the Atlas.

Eventually I set a Google Alert for the War of 1812 and related headings and started reviewing the results regularly. I was finding an interesting trend in the news reports leading up to the commemorative year of War of 1812 "celebrations": There were many stories of towns, townships, municipalities and regions being funded to put together commemorative events. Such events would in turn bring tourism revenue into these communities, making the War of 1812 Commemorative year a year for community economic development in addition to the celebrations. The missing pieces to this cartographic puzzle were the countless Anishinaabe communities whose ancestors were instrumental in Britain's self-acclaimed victory in that war. In contrast with the voluminous amount of news I was receiving multiple times a day regarding newly awarded funding to community $\mathrm{X}$ or township $\mathrm{Y}$, there was barely a mention of funded commemorative events in Anishinaabe communities; although, as I heard through many grapevines, funding had been applied for, but not received.

Finally, on October 11, 2012, the opportunity came to include some "live" Anishinaabe content in the War of 1812 Map when I video recorded the "Four Wars of 1812" Roundtable discussion. Alan Corbiere (referred to earlier), Program Coordinator for the Anishinaabemowin Revitalization Program at Lakeview Elementary School in M'Chigeeng, had been invited to represent the Anishinaabe perspective (although in the program it is referred to as the "Native American" perspective). Alan was joined by Dr. Sid Hart, Senior Historian at the Smithsonian's National Portrait Gallery to give the American perspective; Dr. 
Andrew Lambert, Laughton Professor of Naval History in the Department of War Studies at Kings College, London, to provide the British perspective; and, Dr. Peter MacLeod, pre-Confederation historian at the Canadian War Museum and curator of 1812 represented the Canadian perspective (see Appendix D for digital audio sample from the event featuring a War of 1812 story by Alan Corbiere).

As I listened, watched and recorded, I could not help but noticing the differences in approach to history and understanding between Alan's introductory talk and the talks of the others. In general, the western scholars personified their nations and talked in broad and general terms about military and political leaders, and battles and outcomes, while Alan told a particular story involving particular actions, events and people.

\subsection{The Vidal Anderson Investigative Commission of 1849 Map}

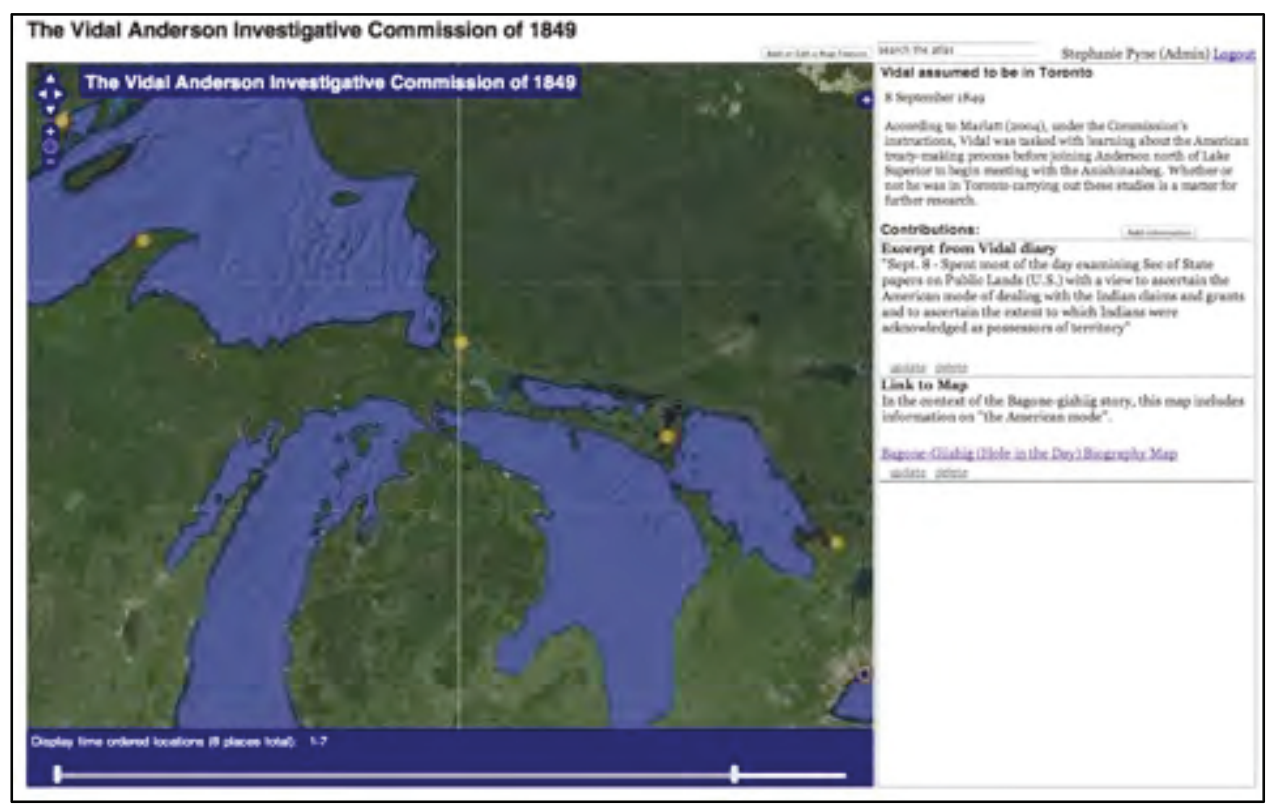

Figure 9.15. Screenshot showing some of the stops Commissioner Vidal made on his way to the Commission meetings in the Lake Superior region. 
The heading "Tracking the Commission" reflects the deconstructive concern in this Atlas for critically examining the records of history. Similar to the Survey Journeys Maps, this map takes a deconstructive geonarrative approach. The process template of geo-transcribing, which emerged through the Survey Journeys mapping process in Phase I, could be used to approach the Vidal Anderson Commission Report. The vision for further development of this map is to involve others in its development from the ground up. This will involve archival research to obtain copies of the relevant documents, and beginning to geo-transcribe them.

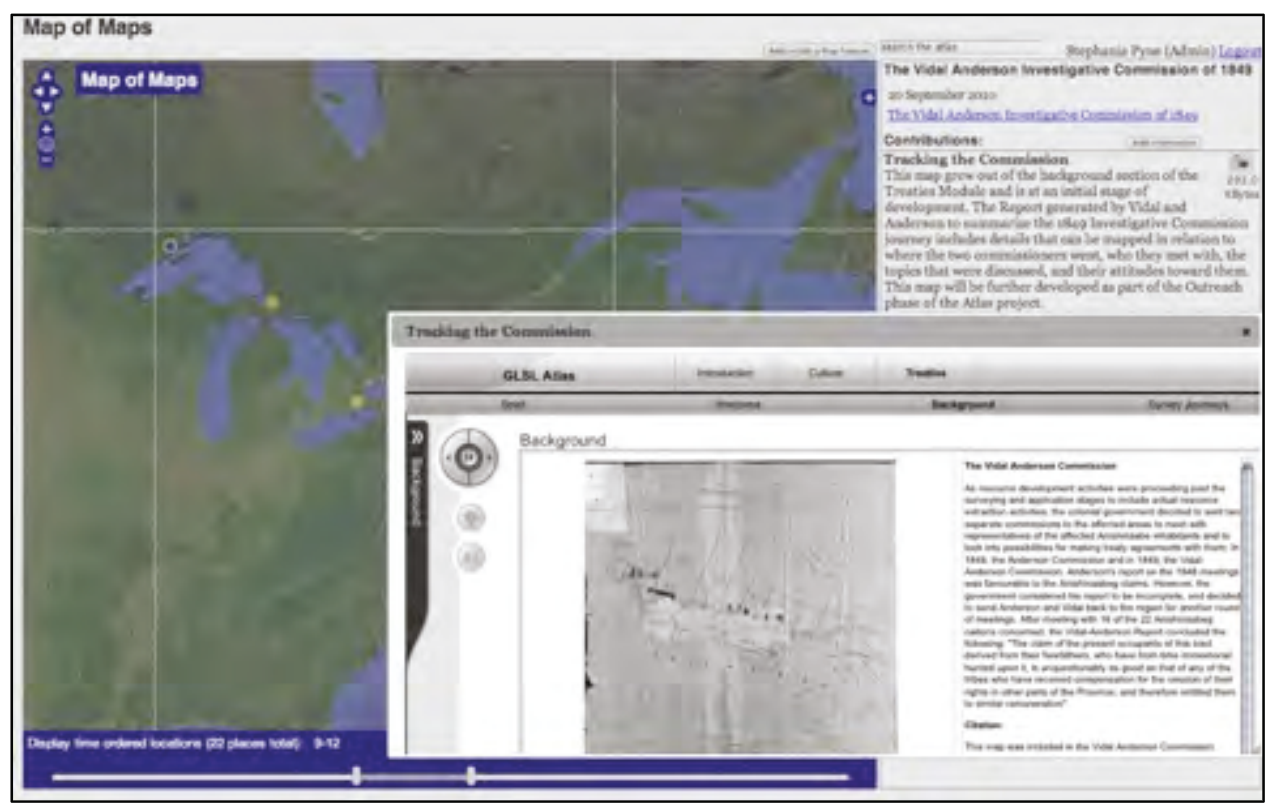

Figure 9.16. Screenshot showing some of the stops Commissioner Vidal made on his way to the Commission meetings in the Lake Superior region, with a pop-up image of the Vidal-Anderson Commission frame in the Background section of the Treaties Module.

The Map of Maps gives a good summary of the Vidal Anderson Commission Map:

This map grew out of the background section of the Treaties Module and is at an initial stage of development. The Report generated by Vidal and Anderson to summarize the 1849 
Investigative Commission journey includes details that can be mapped in relation to where the two commissioners went, whom they met with, the topics that were discussed, and their attitudes toward them. This map will be further developed as part of the Outreach phase of the atlas project.

The opening remarks to the map itself draw attention to the significant role the pre-treaty Vidal-Anderson Commission played in the treaty process:

Welcome to the Interactive Map of the Vidal-Anderson Investigative Commission, which begins to track the travels in 1849 of Commissioners Vidal and Anderson through the Lakes Huron and Superior regions as they met with representatives from 16 out of the 22 Anishinaabe communities that they had identified. This Commission, and the Anderson Commission previous to it, was struck to look into the potential scope, terms and conditions for treaty making with the Anishinaabe in these regions. These commissions set the stage for the Robinson Treaties and the three years of surveying that followed the Treaty signing.

\subsection{The Governance Paper Trail Map}

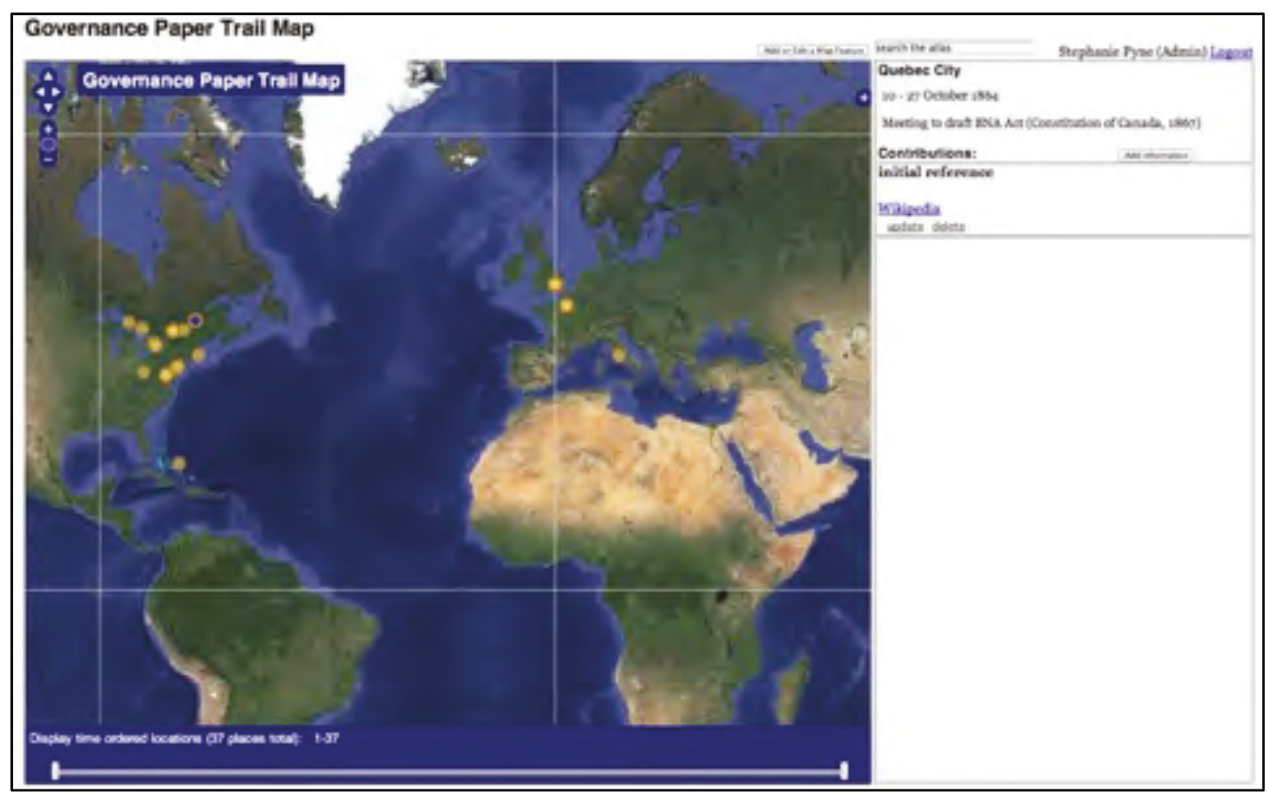

Figure 9.17. Screenshot showing most of the points on the Governance Paper Trail Map.

After our first meeting in August 2007, Patricia Kennedy and I continued to have quarterly meetings, during which we discussed many things, including 
some of the details of the Marlatt chapter. Patricia pointed out the many archival research questions that revolved around the Marlatt chapter; and, as a result of our discussions, the legislative or governance paper trail map idea was born. The mapping in this case would involve extensive archival research tracing the document trail that flowed through the treaty investigation, signing and survey processes. The potential for detail in a project like this was immense. We have continued to meet over the years, and are getting closer to being able to implement a governance paper trail mapping exercise. As with the Vidal Anderson Commission map, a collaborative research project involving students or other interested people is envisaged.

Although there is no summary included for the Governance Paper Trail Map in the Map of Maps, the Welcome text to the Governance Trail Map summarizes its main intent:

Welcome to the Governance Paper Trail Interactive Map, which is intended to share stories related to the nature and role of governance-related institutions in the Lake Huron Treaty process and beyond. The map is intended to track at least some of the legislative and policy paper trail that attended the treaty conception, crafting and signing processes, in addition to the survey process itself. Including archivist Patricia Kennedy in the map research and design process provides knowledge of the contents of treaty-related documents, in addition to the movement of these documents across a specific time and between the spaces of colonial administrative offices. This map is also intended to serve as an archival finding aid and a teaching in archival research strategies. 


\subsection{The Gathering Places Map}

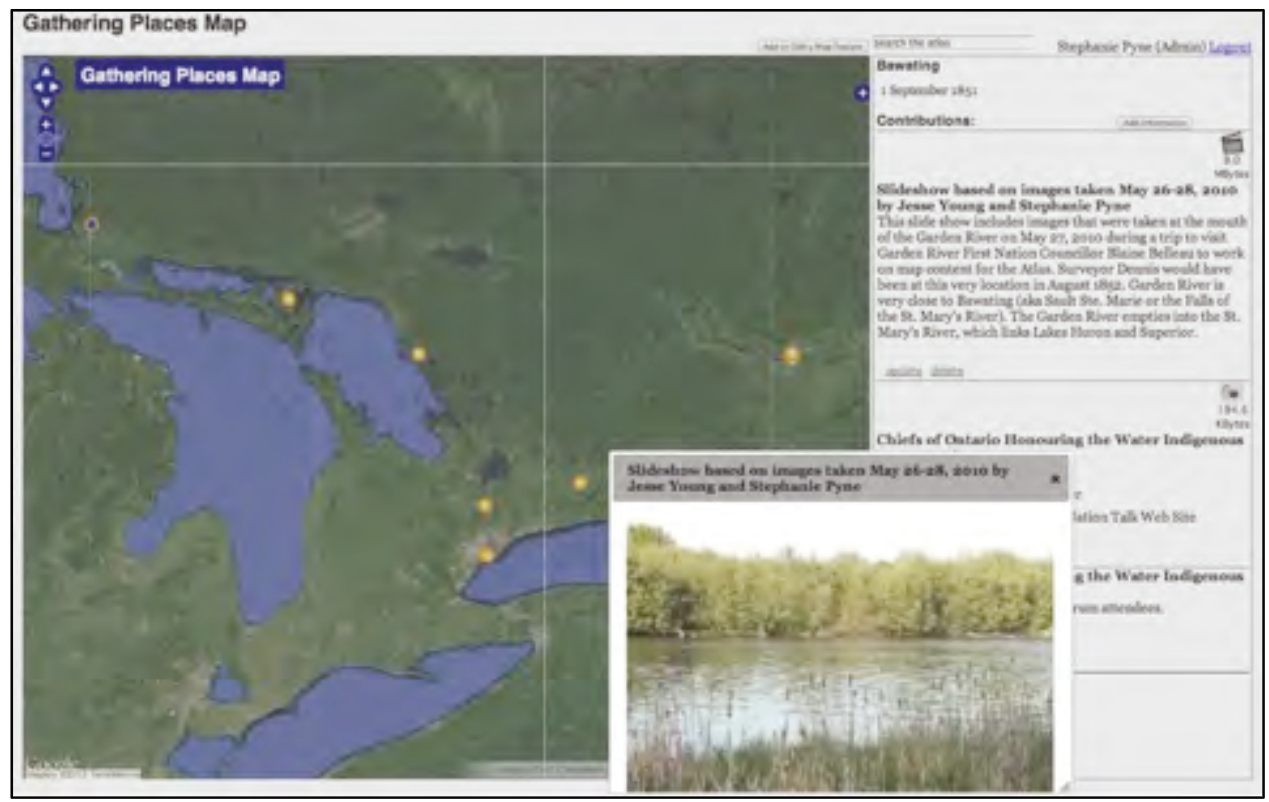

Figure 9.18. Screenshot highlighting the point in blue for Bawating, a traditional gathering place with a long history, with pop-up image of Mouth of the Gitigaanziibing (near Bawating). ${ }^{27}$

The Map of Maps includes the subheading "Meetings over time" to describe the Gathering Places map in order to emphasize the trans-temporal focus of this map. This leaves the map open to welcomingly accepting stories about gatherings that were held long ago at places like Asinabka (Victoria Island, Ontario) and Bawating (Sault Ste. Marie, Ontario), in addition to more current stories, for example video footage I gathered of the December 2012 and January 2013 Idle no More and Chief Theresa Spence events at Asinabka. Footage for these events has already been added to the Gathering Places Map at the location for Asinabka. This on the ground footage provides an alternative to the mainstream media that was circulating at the time; it offers a different perspective of these relatively current events in a way that ties them to their

\footnotetext{
27 See Appendix D for a slideshow of the Gitigaan-ziibing.
} 
history: Mapping current events on the Island together with stories about Asinabka as an international gathering place since time immemorial.

The summary for this map provided in the Map of Maps draws attention to the historical and cultural significance of these gathering places:

There were a variety of places where People from different places on Turtle Island would gather. Some of these same places were later locations for treaty signing events with settler government officials. These treaties often specified that the settler government must give Presents and or annuities annually to the Nations referred to in them. The government officials tasked with dispensing the Presents or annuities often chose traditional gathering places and times to carry out their duties. The point for this Map is placed near the place where the Treaty of Niagara meetings were held.

\subsection{The Community Stories Map}

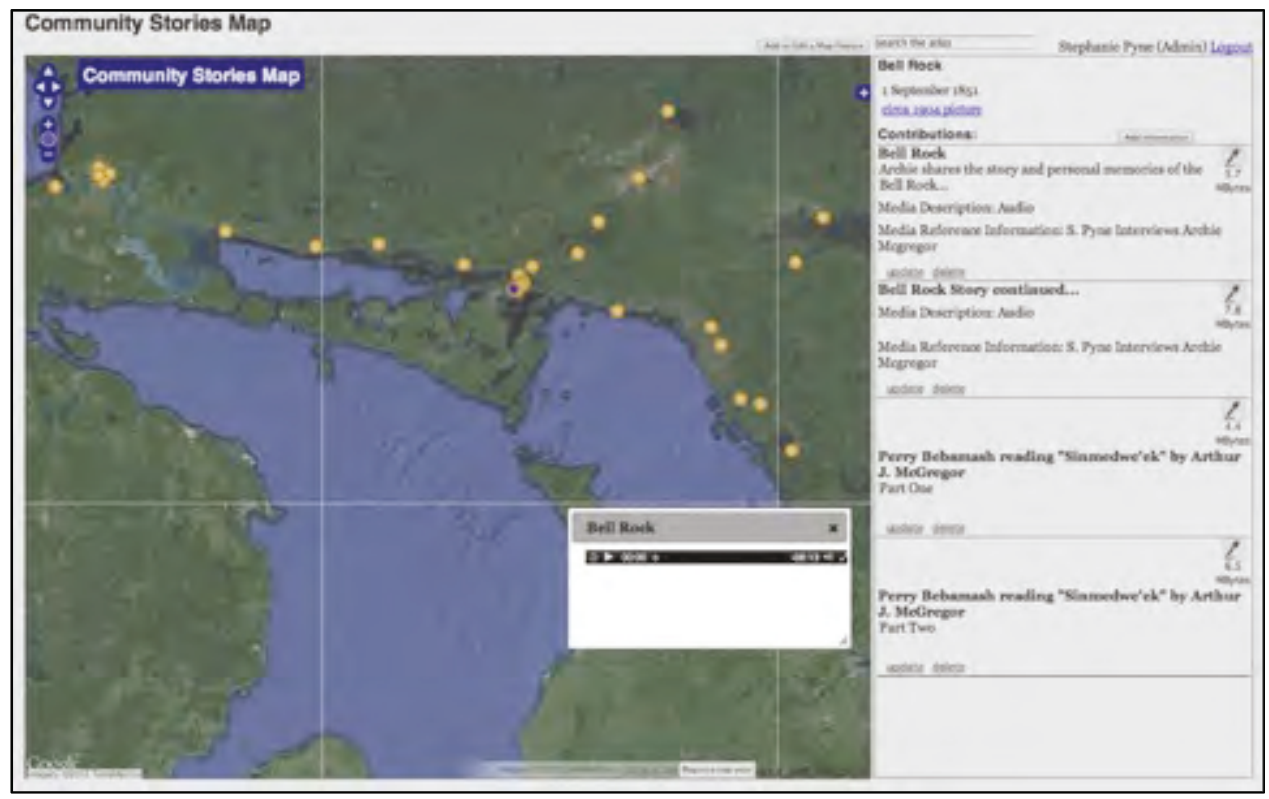

Figure 9.19. Screenshot highlighting point for the Bell Rocks with audio recordings of meetings with the late Arthur J. (Archie) McGregor and voicerecorded translations by Perry Bebamash reading from Archie's "Sinmedwe'ek", showing pop-up window for Archie telling a story about the Bell Rocks.

Although there is no summary yet provided for this map in the Map of Maps, the 'Welcome' text to the map provides a general summary: "Welcome to the Community Stories Interactive Map, which is intended to share some stories 
from community members and those published by online news services related to communities in the Lake Huron and Superior regions".

The introduction also draws attention to a particularly significant community contribution: "Highlights in this map include audio recordings of the late Archie McGregor made in February 2008 when Archie talked about the Bell Rock and Dreamer's Rock. There are also audio clips of Perry Bebaamaash reading from Archie's Wiiswaaskingaa (Land of the Birch Trees) about Sinmedwe'ek (the Bell Rock) and Enjibwaajigeng (Dreamer's Rock)."

\subsection{The Residential Schools Map}

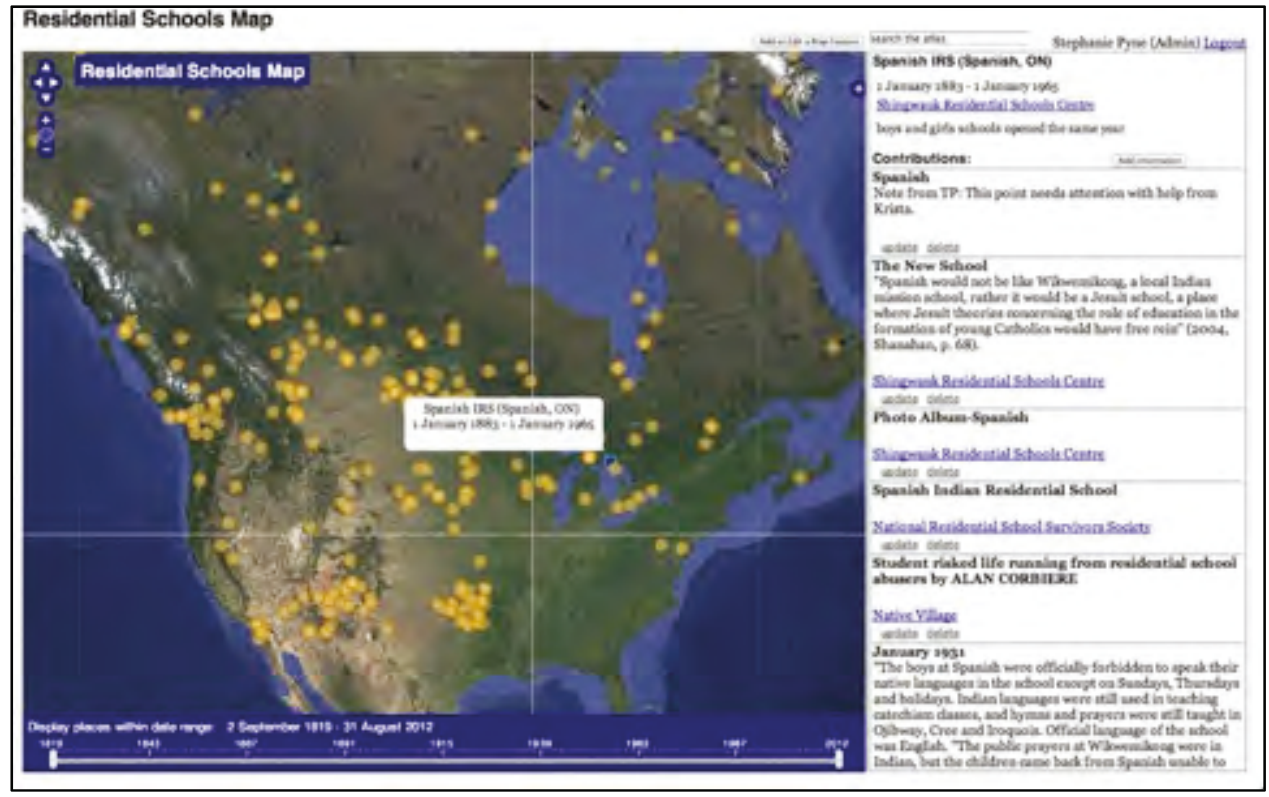

Figure 9.20. Screenshot showing most of the points on the Residential Schools Map for North America, highlighting the point for Spanish Residential School, which - although in the Lake Huron Treaty region - was attended by children as far east as Quebec.

Although there is no summary available for this map in the Map of Maps,

the 'Welcome' portion to the map itself provides some relevant background:

Welcome to the Residential Schools Interactive Map, which is the first example of a Lake Huron Treaty Atlas map being made remotely, in a separate location from the Geomatics and 
Cartographic Research Centre, the Atlas host. This map is an initiative of the Shingwauk Residential Schools Centre (SRSC or Centre) of Algoma University, located on the site of the former Shingwauk and Wawanosh Indian Residential Schools in Sault Ste. Marie, Ontario. The Centre, formerly known as the Shingwauk Project, is a cross-cultural research and educational development project of the Children of Shingwauk Alumni Association (CSAA) and Algoma University (AU). The Project was founded in 1979 by former students and friends of the Residential Schools and Algoma faculty members who were inspired by Chief Shingwaukonse's Vision of the "Teaching " and his grandson Dan 's directive:

"The School never closed. It just entered a new phase of development. It has to be given a chance to finish what it started. It has to put back what it took away. It will be the people who went there that will care. Bring them together. Let them gather and they will know what to do." (The Shingwauk Project)

The Residential Schools Interactive Map is being built as a "Healing and Reconciliation through Education" resource as we work together to address the impacts of Canada's Residential Schools. Through the Map's interactivity, we will contribute to its development as we proceed on our journey of "healing and learning" and rebuilding relationships in Canada together.

The Map includes information and resources on Residential Schools in the United States where similar policies were employed and where addressing the impacts is also just beginning; and, it offers a portal into a world of internet-based resources that can be used for information sharing and education, public and private. After almost one year of development from its early conception phase, the Residential Schools map is beginning to form into a space with contacts and opportunities for networking on the Schools and related issues.

The Residential Schools Interactive Map has thus far been built collaboratively by Centre co-op student Tina Priest, working under the supervision of former Centre Director Don Jackson in conjunction with Stephanie Pyne. It will become more complete as the Outreach Phase of the atlas project continues into 2013. As the map develops, please feel free to browse through.

The inspiration for this map was born during the first week of April 2011, during a visit to Bawating (Sault Ste. Marie) to join the students in their final week of innovative B.A. program in Anishinaabe Studies hosted by Shingwauk Kinoomaage Gamig (University). As I was walking across the grounds of Algoma University, the former Shingwauk Residential School, someone I had just met suggested that I also meet the people working at the Shingwauk 
Residential School Centre (also at Algoma University). Almost immediately after this, I met Tina Priest, an intern working with the Shingwauk Residential School Centre. I suggested that if the Centre was interested, it could have a virtual map to supplement the large map on the wall in front of us, full of thumbtacks representing schools. This initial conversation evolved into a working partnership between the Shingwauk Residential School Centre and the Geomatics and Cartographic Research Centre, a partnership that has involved many generative stories.

\subsection{The E.F. Wilson Biography Map}

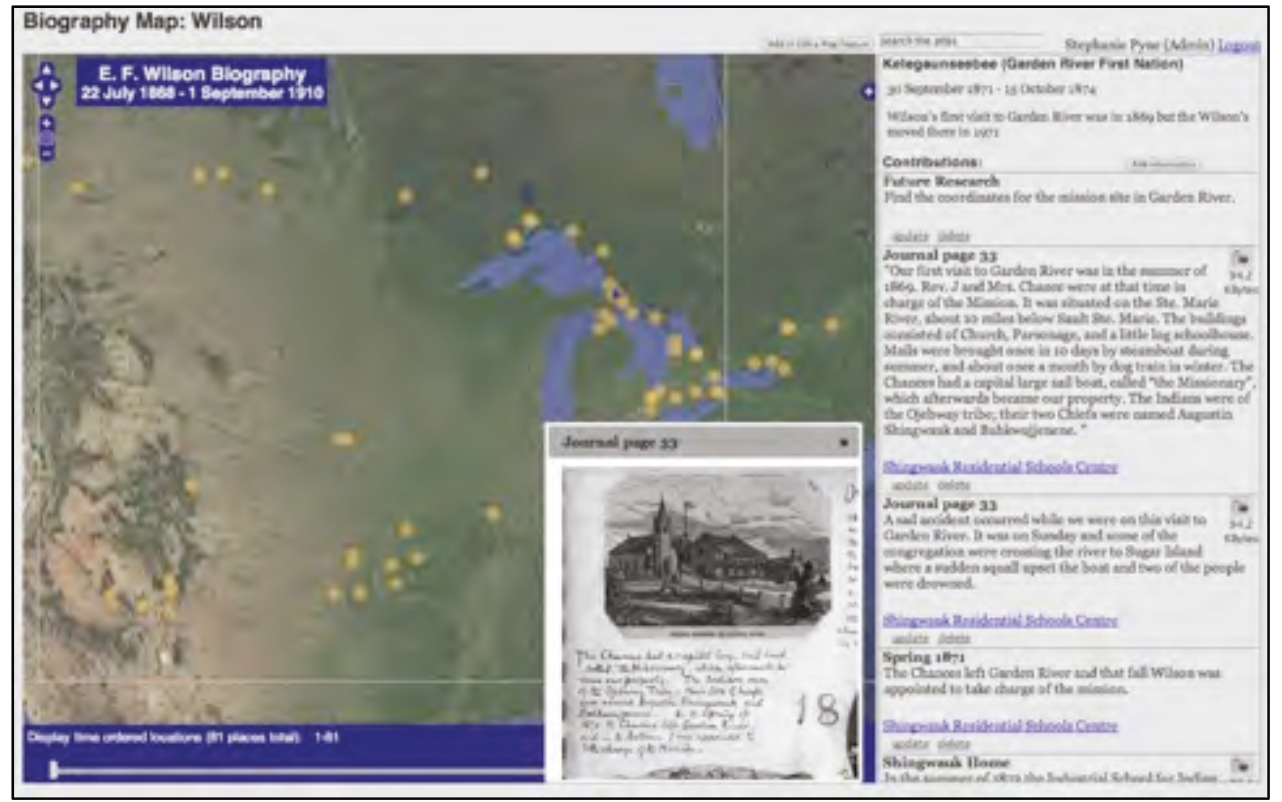

Figure 9.21. Screenshot showing point for Gitigaan-ziibing (Garden River) in blue, with pop-up image of digital scan from E.F. Wilson's Journal, in addition to points for many of the places E.F. Wilson lived and visited.

This map was born out of talks between Don Jackson, the former Director of the Shingwauk Residential School Centre, during which he shared with me copies from E.F. Wilsons's sketchbook, which he had photographed during the 
eighties while visiting some of E.F. Wilson's descendants. E.F. Wilson had been the Principal of Shingwauk Residential School in its early days.

Although there is no summary available for this map in the Map of Maps, the following 'Welcome' to the map itself provides some relevant background:

Welcome to the EF Wilson Interactive Biography Map, which contains digital images of the Illustrated Journal of Reverend Edward Francis Wilson (1844-1915). The map's initial goal is to share some of the biographical events of E.F. Wilson's life across time and space in order to shed more light on the character and relationships of a man who worked with Chiefs Augustin Shingwauk and Henry Buhkwujjenene to build the Shingwauk and Wawanosh Industrial Schools in Sault Ste. Marie and other Indian Schools across Canada. He was also known as "Puhgukahbun"(Clear day light) and "Sahyahquadung" (the Proclaimer) by the Ojibway. Wilson was principal of the Shingwauk and Wawanosh Homes from 1873-1893, and also built schools at Sarnia, Garden River, Batchewana, Nipigon, Elkhorn (Manitoba), and Medicine Hat (Alberta) and travelled extensively throughout Canada and the United States visiting and studying Indian languages, cultures and education.

The map is based on the Autobiographical Journal and related works, which EF Wilson compiled and illustrated over many years, and which are housed in original or copy at the Shingwauk Residential Schools Centre at Algoma University (formerly the Shingwauk and Wawanosh Indian Residential Schools) in Sault Ste. Marie, Ontario.

This map of Wilson's life travels, work and relations is being built collaboratively by Shingwauk Residential Schools Centre coop student, Tina Priest (who is working remotely from Sault Ste. Marie) under the supervision of Don Jackson and in conjunction with Stephanie Pyne. It may incorporate video, images and text in addition to some sound clips and links to related sites, and it will become more complete as the Outreach Phase of the atlas project continues into 2013. As the map develops, please feel free to browse through.

We included Tina in the discussions of how to begin constructing the E.F. Wilson Biography map, and Tina took off with it, mapping 81 points and discovering some interesting questions along the way. 


\subsection{The Carl Beam Map}

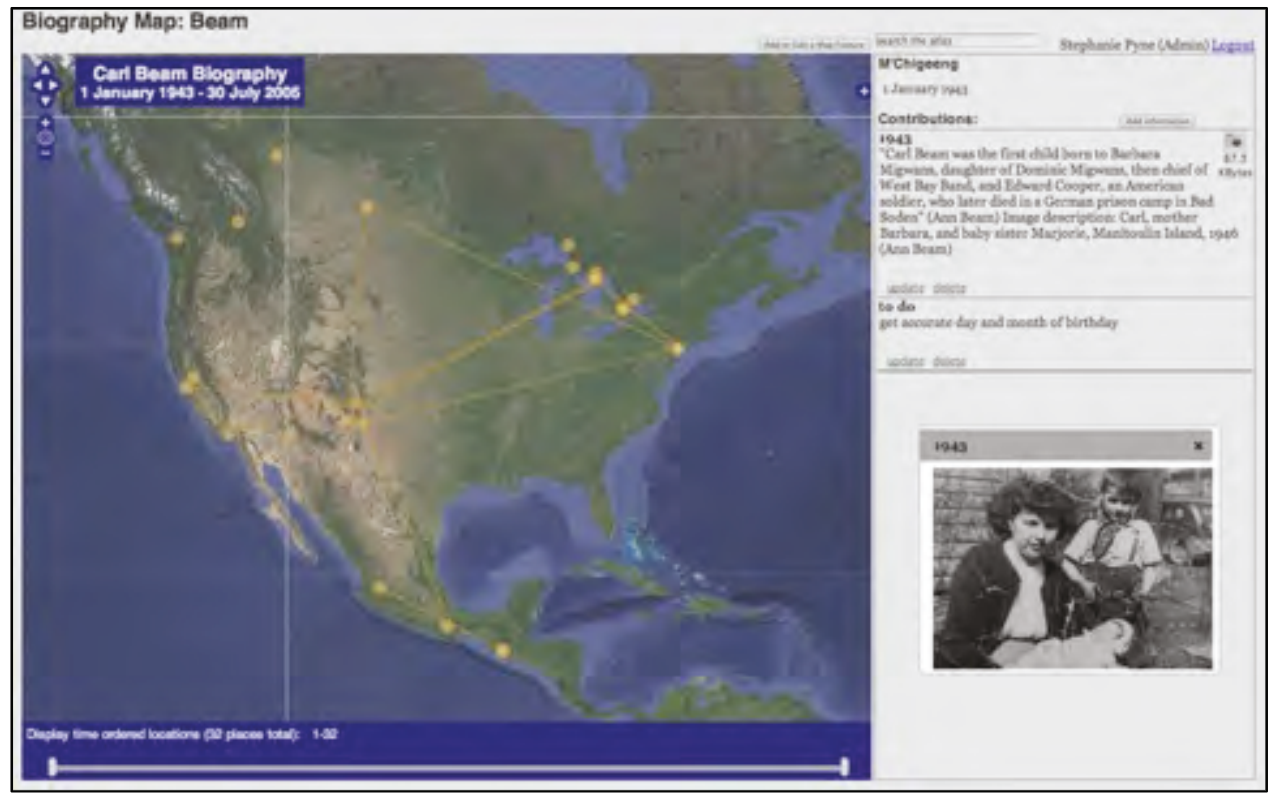

Figure 9.22. Screenshot showing points for places lived and travelled by Carl and (later) Ann Beam, with pop-up image of Carl as a young boy with his mother. Based on biographical essay by Ann Beam. ${ }^{28}$

The Carl Beam Map was inspired by the keynote speech by Greg Hill, Audain Curator and Head of the Department of Indigenous Art at National Gallery of Canada, at the 2010 Ojibwe Cultural Foundation Conference, "Anishinaabewin: Modes of Knowing, Ways of Life". In addition to the fact that Carl Beam was from the Lake Huron Treaty region - M'Chigeeng on Mnidoo Mnising - his innovative approach to decolonizing art and questions of identity fit in perfectly with the narrative flow of the Atlas.

Although there is no summary provided for this map in the Map of Maps, a detailed summary is provided in the 'Welcome' to this map:

Welcome to the Carl Beam Interactive Biography Map, which began with the idea of sharing the perspectives of the

28 See the following link to Neon Raven Art Gallery for more information about the work of artists Carl and Ann Beam, and Anong Migwans Beam: http: / / www.neonravenartgallery.com/Welcome.html 
authors published in the Carl Beam Catalogue produced by the National Art Gallery (2010). The first essay to be mapped is "The Life and Work of Carl Beam: a Narrative Chronology", written by his wife and fellow artist, Ann Beam.

\section{$[\ldots]$}

\section{Essay Introduction: Carl Beam Chronology:}

"In assembling this chronology, I have drawn from notations in Carl's sketchbooks, from his collection of catalogues and correspondences, and from my own voluminous sketchbook and travelogue record. In going over this material, I am astounded by how much excellent, high-quality art Carl created. In stellar terms, he was a first magnitude star. The sheer brilliance and volume of his large-scale works, often created under difficult circumstances, is inspiring. His art always contains that unusual, un-nameable "something" that engages us, inspires us and takes us a little bit forward in our understanding of our current position. The excitement and continuity of his creative process, was revelatory to witness. As his wife and fellow artist, I was privileged to not only share my life with his, but also to witness his creative process and assist him with his projects for twenty-six of his thirty years as a practising artist. He respected "real art practice." He took himself seriously as an artist, and never doubted his work. He always worked through to completion on a project. "Work first, eat later," he would say.

Carl achieved this remarkable volume of art production against a background of constant travel and fluctuating financial circumstances. We went everywhere together, taking as many as four major trips a year. Our travel was always related to our work, either to attend an exhibition or symposium, or to pursue the joy of continual exploration of places, art materials and art ideas. The majority of these expeditions were long cross-country road trips, Carl and Anong and me in our station wagon packed with art supplies, a frying pan, a guitar and an accordion. We had plenty of time to talk and assimilate ideas. These journeys fueled the excitement of living, and the excitement of living, fueled the art". 


\subsection{The Nanboozhoo Mindemoye Story Map}

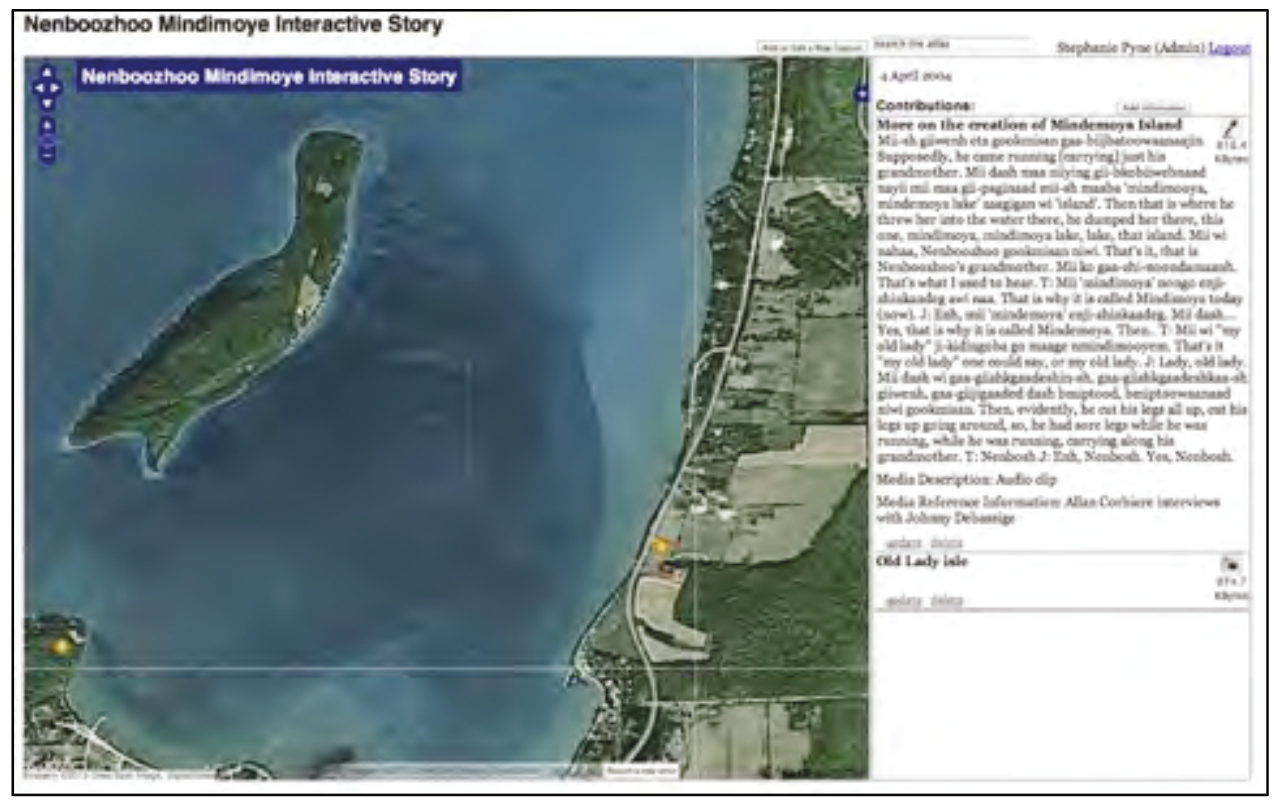

Figure 9.23. Screenshot showing two stops in the journey of Nanboozho when he threw his grandmother into the lake and she became Mindemoye Island.

The Welcome portion of this map provides a good introduction:

Welcome to the Nenboozhoo Mindimooye Story Map, which has been migrated to the Lake Huron Treaty Atlas from the Culture Module of the Atlas of Indigenous Perspectives and Knowledge. The map tracks the story of Nenboozhoo and the creation of Mindimooye M'nsing (Old Lady Island), and includes audio recordings from a March 13, 1999 interview between M'Chigeeng elders Johnny Debassige and Alvin (Ted) Corbiere, and Alan Corbiere.

This map is just beginning and will become more complete as the Outreach Phase of the atlas project continues into 2013. As the map develops, please feel free to browse through. Comments and questions are welcome and can be sent to Stephanie Pyne at lakehurontreatyatlas@gmail.com.

This map was 'migrated over' to the Lake Huron Treaty Atlas from the

Culture Module of the Atlas of Indigenous Perspectives and Knowledge. Further development of this map is on the agenda throughout phase III and subsequent phases of the atlas project. 


\subsection{The Language and Culture Map}

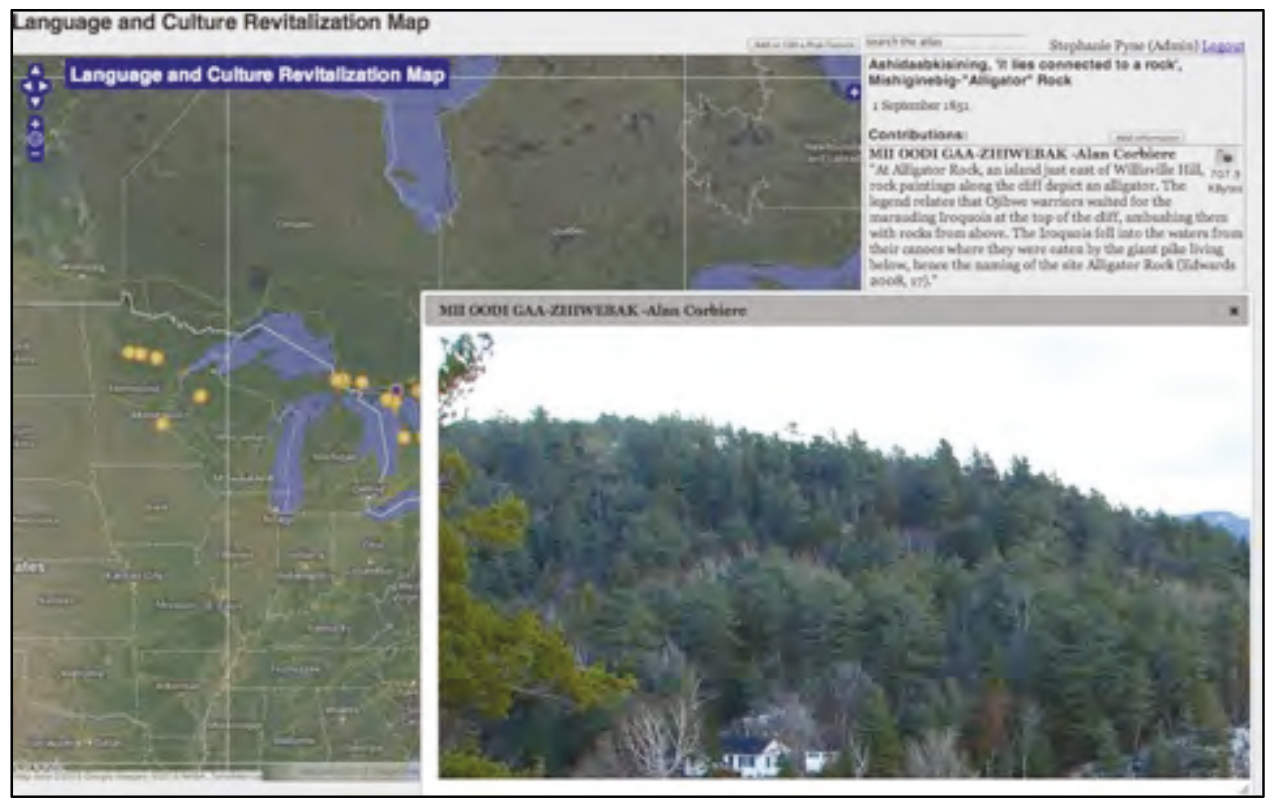

Figure 9.24. Screenshot showing the point in blue for the location of Miioodigaazhiwebak (Alligator Rock). Photo by Alan Corbiere.

The simple sub-heading "Initiatives" is used in the Map of Maps to introduce the Language and Culture map as just that, a place where knowledge about beneficial Anishinaabe initiatives aimed at promoting and restoring culture and language - initiatives - can be shared. The summary for this map emphasizes the link between the Anishinaabe and the water: "Anishinaabe language and culture initiatives are ubiquitous throughout the Great Lakes region and beyond. The point for this map is placed in the waters of Lake Huron to reflect this". There is much potential for this map to develop in the future. 


\subsection{The Current Affairs in the Lake Huron Region Map}

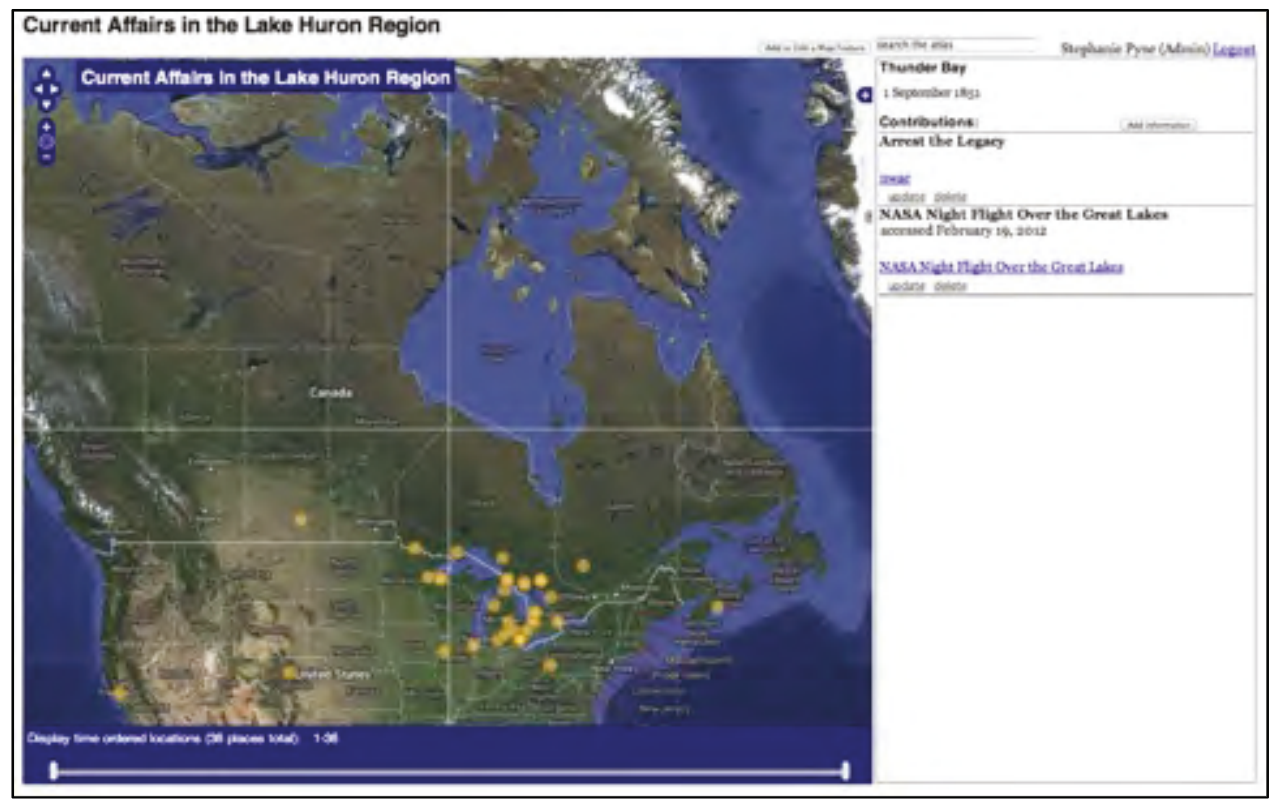

Figure 9.25. Screenshot showing points for news and current affairs in, around and about the Lake Huron Region.

The Map of Maps provides the following summary: "This map was inspired by a presentation on mapping ideas. The point for this map is located in the water because it is so central to the current affairs in the region. The Map contains links to stories about events in the present, which can be compared with stories about events in the past in the historical maps".

The map itself provides the following introduction: "Welcome to the Interactive Map of Current Affairs in the Lake Huron Region, which shares stories published by online news services related to Lake Huron regional issues, many of which are related to water, the environment and the health of communities. Some of the stories reflect an Anishinaabe perspective but many do not". 


\subsection{The Treaty and Consultation Current Affairs Map}

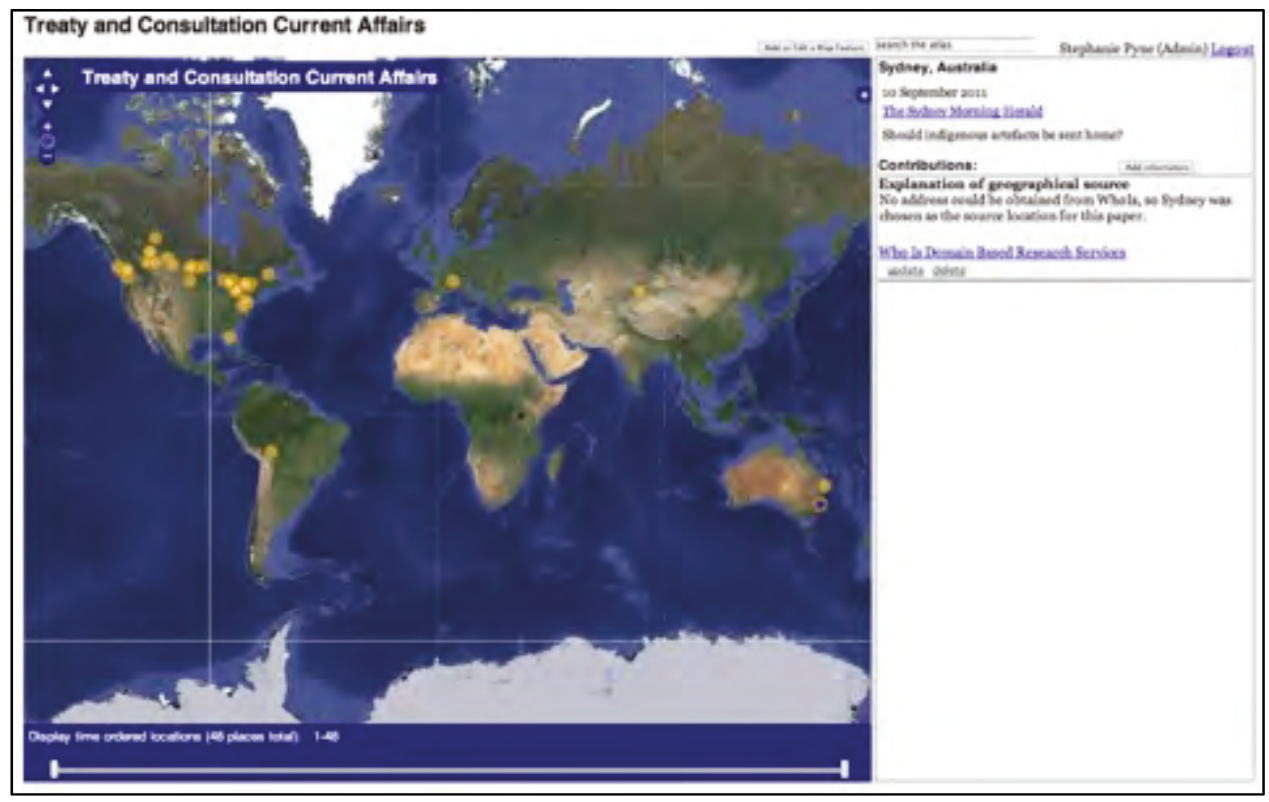

Figure 9.26. Screenshot showing broad scope of interrelated treaty and consultation current affairs.

This news-clipping map is intended to emphasize the "living" aspect of treaties. It is international in scope and includes stories related to Indigenous peoples, treaties and consultation. The point for this map is located at the place where the two-row wampum belt was exchanged in 1613. The map includes the following welcome message:

Welcome to the Treaty and Consultation Current Affairs Interactive Map, which is intended to share stories published by online news services related to a broad range of treaty- and consultation-related issues across time and space. The stories are the results of an ongoing online search for terms such as "aboriginal treaty" and "aboriginal consultation".

Mapping these stories provides knowledge of current treatyand consultation-related issues and trends, which includes some knowledge of Nation-to-Nation relationships today. This map is just beginning and will become more complete as the Outreach Phase of the atlas project continues into 2013. Please feel free to browse through as work on this map progresses. 
While this map casts a broad net, collecting stories over time from across the world and related in many ways to treaty and consultation issues, these issues are relevant to a full understanding of the Lake Huron Treaty story when it comes to sharing knowledge and experience. The idea for this map and the other news-clipping style maps came from an International Cartographic Association presentation I had seen on mapping ideas. Having a strong concern with applying cartographic approaches to understanding assumptions, with an interest in trends, I began to construct the news-clipping maps to track ideas along the various themes indicated above. I have worked with Tina Priest on the early stages of a Residential Schools news-clipping map, which will be uploaded to the Atlas after it is converted to the new version of the Nunaliit II software framework.

\subsection{Works in Progress - Concluding Remarks}

Each map in the Lake Huron Treaty Atlas is a working pilot project and an invitation for future development. At least one full chapter could be devoted to a discussion of each map individually in terms of describing its iterative development, in addition to challenges and opportunities for the future. In this regard, the Map of Maps could be used in the future as the place to document this sort of information, since there is a point for each map in this mapped portal.

Although the content of each map is specific to that map to some extent, all of the maps in the Atlas are interrelated to various degrees. The Map of Map represents one attempt to draw attention to some of these relationships through the placement of the points for each map across space and over time. However, it only reflects the maps that were in existence prior to the launch of the Lake Huron Treaty Atlas near the end of phase II of the project. Work toward designing a 
series of new maps has begun in phase III, but is not yet reflected in the Map of Maps. One such map, which is still in the conceptual phase of development, is the dynamic map portal idea. Work to develop this map depends on the outcome of research to develop a dynamic concept map portal for the cybercartographic Siku (Sea Ice) Atlas, and provides a good example of inter-project relationships in cybercartography. The design vision for this dynamic portal to the other maps in the Atlas is that it not be tied to a conventional map background, and that as new maps are added and their relationships to one another change, their placement in the dynamic concept map portal will change to reflect this. 


\section{Part V Dancing into the Future - Building Awareness to Bridge Relationships}

Part V offers some reflections that elaborate on key bridging concepts that have been developed through this thesis research, in addition to comments regarding ongoing challenges and possibilities for the future. 


\section{Intercultural Bridging: Iterative Development and Emergent Mapping Practices}

This chapter comments on some of the bridging templates that have emerged through the iterative atlas-making processes to date. These templates are consistent with Linda Tuhiwai Smith's (1999) decolonizing methodologies. For example, the construction process for the pilot version of the Treaties Module was exploratory; it was not 'preordained' by any western scientific method, but remained open to the insights and directions of the Anishinaabek from the Treaty's signatory communities, and involved the development of relationships in a rhizomatic manner (Caquard et al., 2009; Pyne and Taylor, 2012).

\subsection{Reflexivity}

Writing reflexively and telling stories about the iterative processes in the making of the Atlas maps can be considered as a form of map performance that enriches people's understandings of the maps. Some of these stories are already included in the Travels in the Making of the Atlas Map, which begins to document a diverse array of Atlas-making trips and activities, thus making the experiences not only part of the process of map-making but also a part of the maps themselves.

The narratives presented in both chapters 8 and 9 involve drawing on the narrative logic of the maps they interpret. Although both chapters involve telling stories about the making of the maps, they do so in distinctive ways. In chapter 8 , the narrative follows my travels as sketched out in the Travels in the Making of the Atlas Map, which steers the narrative in a certain direction. In chapter 9, the narrative follows the geonarrative logic of the Map of Maps, which guides the chapter narrative down the path of map intent and development process details. 
Reflexivity is demonstrated in the way these narratives bend back upon the maps they interpret, both drawing on their form and contributing to their understanding.

There is a cascading pattern of the reflexivity in the relationships between these narratives and the maps they interpret. In the case of chapter 8 , the text reflexively examines the Travels in the Making of the Atlas Map; this map in turn reflexively refers to the other maps in the Atlas, which themselves reflexively examine the themes they "geonarrate". Laura Ellingon's (2009) "multigenre crystallization", referred to in chapter 3, is a good descriptor for the relationship of cascading reflexivity that is evident in the relationships between the atlas maps and this thesis, especially insofar as the crystal has a fractal structure and emergent growth patterns (Ellingson, 2009; Mandelbrot, 1985).

\subsection{Performance}

The many iterative processes involved in the Atlas-making process provide the best examples of the performative aspect of the Atlas. Many of these processes involve the kind of "talk" referred to in chapter 7. A performative or processual approach to map and atlas making is central to this project (Caquard et al., 2009; Pyne and Taylor, 2012; Pyne, 2013). For example, the process of writing this thesis goes beyond the thesis itself and is part of the broader Atlas design and development process, in addition to being a piece 'about' the Atlas (Wood and Fels, 2008). From this perspective, the atlas is an ongoing process oriented toward contributing to the conditions for sufficient intercultural mutual understanding. The atlas production process involves a variety of interpersonal interactions, knowledge exchanges and actions related to 'telling the Lake Huron Treaty story' through atlas design, development and use. The line between the 
Atlas as a material object and the design and development processes involved in its making are blurred, the dichotomy is diminished. Insofar as the Atlas is being designed to allow for ongoing critical input and contributions, the map user can also become the mapmaker. In this respect, designing, developing and using the atlas are all intertwined, and work is progressing to design the digital architecture that will allow people to add their own comments and media to the Atlas maps (Brauen et al., 2011).

\subsection{Relational Space}

The original aim of this project as stated in my thesis proposal was to provide an example of relational space in an effort to bring to the surface some of the meanings buried deep within the literature. As Doreen Massey admitted in 2004, "thinking space relationally has become one of the theme-tunes of our times in geography. It is, as with so many things, more easily cited in general than excavated in practice. "(3).

One way this project has begun to make relational space more "real" is by incorporating relational space thinking into the geonarrative design and development process: Applying the theory. John Allen's (2004) contribution to the J.W. Keating and J.S. Dennis Biography Maps discussed in chapter 9 provides a good example.

Throughout this past seven years of collaborative mapping engagements, a growing web of relationships has emerged between individuals with intersecting interests, making the Atlas community itself, another significant aspect of relational space that can also be mapped. 


\subsection{Narrative}

It is difficult to conceive of a product in processual terms, which is exactly what Kitchin and Dodge (2007) are asking people to do with their ontogenetic conception of maps. Storytelling can help with making this conceptual (and perceptual) shift. As a spatialized form of storytelling, cybercartographic geonarrative is comprised of the processes that go into its making and that surround its use. Geo-transcription is a specific form of geonarrative that emerged from a series of iterative processes (described in chapter 8). The concept of "mapping epiphany" arose as I began reflecting upon the experiences involved in the decision that led to the emergence of "geo-transcription" in the Survey Journeys Maps, which in turn became a template for other geonarratives, the E.F. Wilson Biography and the Bagone-Giizhig maps, for example.

Looking at the map as metaphor further emphasizes its reflexive nature. In this regard, the Atlas is like a mask or a shield that reveals rather than hides dimensions that have been previously hidden from view or otherwise not attended to. In Indigenous Storywork, Educating the Heart, Mind, Body, and Spirit (2008), Jo-Ann Archibald discusses her work gathering stories as the creation of a story basket, which reflects the care taken in gathering the stories. The notion of gathering is implicit in the metaphor of the Atlas as both a gathering place and the act of gathering, which has been and can be further informed by Anishinaabe teachings concerning gathering. ${ }^{29}$

${ }^{29} \mathrm{~A}$ traditional knowledge presentation on medicine plant gathering at the Anishinaabewin: Modes of Knowledge, Ways of Life Conference (2010) by Julie Ozawagosh from Atikameksheng Anishnawbeg provides one memorable example of how my approach to knowledge gathering with the aim of healing knowledge was informed by Anishinaabe teachings. 
Story has functioned as method in the broadest sense (some might refer to this as methodology). The Rainbow Raven and the Fly in the Bottle story has remained as the introduction to the Atlas throughout its iterative development. Broadly speaking, this guiding story provides both the aim (to broaden awareness) and the method: to follow, in a circular pattern. As recounted in chapter 8 , the children at Shawanosowe School inadvertently demonstrated the consistency between this story and a traditional Anishinaabe story by placing the images to the story in the correct order, and by recognizing it as their story. I have felt this story come alive over the years as I have fulfilled my role as geonarrative and content editor: gathering knowledge bobbles (thoughts, ideas, insights, stories, images, video and audio clips and links to websites), and following a circular or holistic pattern to place them in a way that begins to raise both questions and awareness of the many dimensions of treaty relationships.

\subsection{Inclusivity and Experience}

The "geo-transcription" method of documenting archival documents emerged through iterative processes that included Anishinaabe ways, social interaction, learning, mapping epiphanies, and an iteratively developed technology. Cybercartography attempts to achieve inclusion in mapping by allowing a number of different narratives to be presented without privileging any. The Lake Huron Treaty Atlas takes cybercartography in a new direction in this regard by adding a critical edge to its inclusiveness. Not all perspectives and understandings of the Lake Huron Treaty historical geography are being included in the research behind the development of the module. In an effort to contribute to the reconciliation of Lake Huron Treaty-based relationships today, the Atlas will continue to be developed by incorporating perspectives of people 
from the Lake Huron Anishinaabe communities and critical post colonial perspectives; while the "colonial" perspective will continue to be incorporated only insofar as it is being critiqued. This is consistent with Brenna Bhandar's view that "the project of reconstituting and decolonizing a colonial settler state is reliant upon the creation of new histories, in which certain strands and stories of colonial injustice and dispossession become the predominant ones" $(2004,834)$.

A primary concern for critical cartography is with whether or not cartography is capable of meaningfully conveying such things as experience, Indigenous perspectives and knowledge, and critical academic approaches to the status quo (Johnson, Louis and Pramono, 2006; Turnbull, 2007). The work to create the Lake Huron Treaty Atlas has attempted to address this concern through flexible, responsive and inclusive design and development processes. Its transdisciplinary approach extends beyond academics; and, sharing across knowledge spaces is one of its defining characteristics (Brauen et al., 2011).

The ongoing iterative development of the wiki component of the Atlas, which will allow for a variety of contributions, provides another example of inclusivity in this project. There are several reasons that we have elected to take time and care with respect to developing the wiki component. In part, these reasons relate to technological challenges. In addition, opening up any webbased product to user input gives rise to both opportunities for democratic participation and challenges related to the politics of cyberspace (Crampton, 2003). These challenges include privacy, uneven distribution of resources, (cognitive) accessibility, and epistemological and ontological commensurability.

Incorporating Anishinaabe perspectives is one important way the atlas project exercises inclusivity; doing this has given rise to acknowledging and 
incorporating "mapping epiphanies" as essential aspects of the atlas design and development process. Following the two-pronged or braided approach taken in the atlas project, this concept is consistent with both the critical academic literature on epiphanies (McDonald, 2008) and the Anishinaabe discipline of following your heart. This methodological inclusivity involves recognizing and valuing the ways that people's personalities, predispositions and spirits affect, direct and respond to atlas design and development research processes. The current interest in reflexive approaches to qualitative research is giving rise to new insights in this regard (Denzin, 2012).

Chapter 8 discussed the two instances of significant turning points in geonarrative design, which occurred first in July 2007 and second in January 2008. In the context of the iterative processes involved in geonarrative development, the experiences associated with these decisions functioned as map design epiphanies. Although the concept of epiphany has been studied across various disciplines, including sociology, psychology, literary, and religious studies, and has even extended to design research (Verganti, 2011), much remains to be learned (McDonald, 2008):

Epiphanies [...] are sudden and abrupt insights and/or changes in perspective that transform the individual's concept of self and identity through the creation of new meaning in the individual's life. Epiphanies are momentary experiences of transcendence that are enduring and distinct from other types of developmental change and transformation. Due to this distinction, positive change and transformation that is sudden and abrupt is a relatively underresearched and underdeveloped phenomenon. (McDonald, 2008, 90).

Closer to the idea of mapping design solutions, the term epiphany is rooted in "the ancient Greek word "epiphainesthai," which means to "appear" or "to come into view" (Arnold, 2002) and was used to refer to moments of sudden 
and significant insight (Paris, 1997)" (quoted in McDonald, 2008, 91). Although the narrative details of people's epiphanies vary greatly, certain common characteristics remain not only between these epiphanies, but also in terms of the categories of characteristics listed by different academic researchers across disciplinary perspectives (this suggests that it is a very transportable concept across disciplinary perspectives). Mapping epiphanies share the same characteristics with other epiphanies. Table 10.1 lists common characteristics of epiphanies (McDonald, 2008) with brief descriptions of how the two mapping epiphanies involved in the iterative development of the Survey Journeys maps share these characteristics with other observations of "epiphany".

\begin{tabular}{|c|c|c|}
\hline Common Characteristics & Epiphany 1 - Marlatt Map & Epiphany 2 - Survey Journeys \\
\hline Antecedent State & Antecedent State & Antecedent State \\
\hline $\begin{array}{l}\text { Epiphanies are preceded by } \\
\text { periods of anxiety, } \\
\text { depression, and inner } \\
\text { turmoil (Denzin, 1989, 1990; } \\
\text { Jarvis, 1997; Jenson, 1998, } \\
\text { 1999; Loyttyniemi, 2001; } \\
\text { Miller \& C'de Baca, 1993, } \\
\text { 2001) }\end{array}$ & $\begin{array}{l}\text { A period of uncertainty } \\
\text { preceded the mapping } \\
\text { epiphany in terms of } \\
\text { knowing what and how to } \\
\text { map. Dealing with these } \\
\text { feelings required me to } \\
\text { balance them with the } \\
\text { confidence that answers } \\
\text { would present themselves. }\end{array}$ & $\begin{array}{l}\text { As I started talking with potential } \\
\text { atlas collaborators, I began to } \\
\text { realize the depth of the 'treaty } \\
\text { story'. Although the critical } \\
\text { review of the survey process } \\
\text { provided by the Marlatt chapter } \\
\text { was an excellent geonarrative } \\
\text { resource, I had the feeling there } \\
\text { should be something else. }\end{array}$ \\
\hline Suddenness & Suddenness & Suddenness \\
\hline $\begin{array}{l}\text { Epiphanies are sudden and } \\
\text { abrupt (Beja, 1993; Goud, } \\
\text { 1995; Jarvis, 1997; Jenson, } \\
\text { 1998, 1999; Miller \& C'de } \\
\text { Baca, 1993, 2001; Schultz, } \\
\text { 2001). }\end{array}$ & $\begin{array}{l}\text { The decision to map the } \\
\text { Marlatt chapter came in an } \\
\text { instant as I began reading } \\
\text { through the chapter the day } \\
\text { after receiving the email } \\
\text { from Alan Corbiere via } \\
\text { Heidi Bohaker. }\end{array}$ & $\begin{array}{l}\text { The decision to map the J.S. } \\
\text { Dennis } 1851 \text { and } 1853 \text { survey } \\
\text { diaries came to mind in an instant } \\
\text { as I was driving home after } \\
\text { mapping the Nenboozhoo } \\
\text { Mindemoye story with Alan } \\
\text { Corbiere and Amos Hayes. }\end{array}$ \\
\hline Personal Transformation & Personal Transformation & Personal Transformation \\
\hline $\begin{array}{l}\text { Epiphanies are experiences } \\
\text { of profound change and } \\
\text { transformation in self- } \\
\text { identity (Denzin, 1989, 1990; } \\
\text { Goud, 1995; Jarvis, 1997; } \\
\text { Jenson, 1998, 1999; Miller \& } \\
\text { C'de Baca, 1993, 2001) }\end{array}$ & $\begin{array}{l}\text { Transformation occurred } \\
\text { individually in terms of my } \\
\text { emerging awareness of } \\
\text { history and geography, and } \\
\text { my newfound confidence } \\
\text { and vision as a "mapper". } \\
\text { The Treaties Module also } \\
\text { experienced transformation } \\
\text { as a result of this. }\end{array}$ & $\begin{array}{l}\text { I engaged in a diverse array of } \\
\text { mapping practices while tracking } \\
\text { the surveyor. Learning through } \\
\text { Internet-based resources and } \\
\text { talking with people, the world of } \\
\text { Lake Huron Treaty history and } \\
\text { geography was beginning to open } \\
\text { up to me along many dimensions. }\end{array}$ \\
\hline
\end{tabular}




\begin{tabular}{|c|c|c|}
\hline Common Characteristics & Epiphany 1 - Marlatt Map & Epiphany 2 - Survey Journeys \\
\hline Illumination/Insight & Illumination/Insight & Illumination/Insight \\
\hline $\begin{array}{l}\text { Epiphanies are an acute } \\
\text { awareness of something } \\
\text { new, something that the } \\
\text { individual had previously } \\
\text { been blind to (Denzin, 1989, } \\
\text { 1990; Goud, 1995; Jarvis, } \\
\text { 1997; Jenson, 1998, 1999; } \\
\text { Miller \& C'de Baca, 1993, } \\
\text { 2001; Paris, 1997). }\end{array}$ & $\begin{array}{l}\text { The key insight was the } \\
\text { vision for the map, which } \\
\text { had crystallized at the } \\
\text { moment of the decision to } \\
\text { map the Marlatt chapter. A } \\
\text { related insight emerged in } \\
\text { relation to the multi- } \\
\text { dimensional nature of } \\
\text { treaty processes. }\end{array}$ & $\begin{array}{l}\text { One key insight was the vision } \\
\text { for the map, which had } \\
\text { crystallized at the moment of the } \\
\text { decision to map the J.S. Dennis } \\
\text { survey diaries. Insights into the } \\
\text { links between past, present and } \\
\text { future as constituents of the } \\
\text { 'treaty story' became richer and } \\
\text { further entrenched in my } \\
\text { perspective. }\end{array}$ \\
\hline Meaning Making & Meaning Making & Meaning Making \\
\hline $\begin{array}{l}\text { Epiphanies are profound } \\
\text { insights because they are } \\
\text { deemed significant to the } \\
\text { individual's life (Denzin, } \\
\text { 1989, 1990; Frick, 2001; } \\
\text { Miller \& C'de Baca, 1993, } \\
\text { 2001). }\end{array}$ & $\begin{array}{l}\text { This epiphany was } \\
\text { significant to my life as a } \\
\text { PhD student, in particular, } \\
\text { giving direction to my quest } \\
\text { for a thesis focus, in } \\
\text { addition to being significant } \\
\text { to the life of the atlas } \\
\text { project. }\end{array}$ & $\begin{array}{l}\text { This epiphany was significant to } \\
\text { my life as a PhD student, giving } \\
\text { direction to my quest for a thesis } \\
\text { scope, in addition to being } \\
\text { significant to the life of the atlas } \\
\text { project. }\end{array}$ \\
\hline Enduring Nat & Enduring Nature & Enduring Nature \\
\hline $\begin{array}{l}\text { Although the actual } \\
\text { epiphany is a momentary } \\
\text { experience, the personal } \\
\text { transformation that results is } \\
\text { permanent and lasting } \\
\text { (Denzin, 1989, 1990; Jarvis, } \\
\text { 1997; 2001; Jenson, 1998, } \\
\text { 1999; Miller \& C'de Baca, } \\
\text { 1993, 2001). }\end{array}$ & $\begin{array}{l}\text { The epiphany formed the } \\
\text { basis for an ongoing } \\
\text { approach to geonarrative } \\
\text { that has since been } \\
\text { developed iteratively } \\
\text { around the original core. } \\
\text { Through this epiphanic } \\
\text { process, I was transformed } \\
\text { into a cartographer. }\end{array}$ & $\begin{array}{l}\text { This epiphany resulted in a } \\
\text { cartographically more } \\
\text { sophisticated map than the } \\
\text { original map based on the Marlatt } \\
\text { chapter and gave rise to the } \\
\text { emergence of geo-transcription, a } \\
\text { unique cybercartographic method } \\
\text { that continues to act as a model } \\
\text { for other mapping projects. }\end{array}$ \\
\hline
\end{tabular}

Table 10.1. Common characteristics of epiphanies (McDonald, 2008)) and two Lake Huron Treaty Atlas epiphanies.

Although they are generally quite inclusive, one dimension often missing in transdisciplinary approaches is the phenomenological dimension. The stories of the mapping epiphanies described in this chapter bring together at least two streams of thought in critical cartography: The first is concerned with approaching cartography performatively in terms of mapping processes (Kitchin and Dodge 2007; Turnbull 2007); and the second is concerned with mapping experience (Pearce 2008; Kwan 2007). Without the occurrence of the mapping 
epiphanies in July 2007 and January 2008, there would be no Survey Journeys maps; their role in the map design decision processes was essential.

These two mapping epiphanies were contingent on mapping interactions involving an Anishinaabe community member, and they reflected Anishinaabe perspectives and understanding, in addition to being akin to the Anishinaabe advice to follow your heart. Moreover, the second mapping epiphany followed iteratively upon the first, pointing to the importance of iterative processes in experiential learning. There is a growing literature on the role of emotion in decision-making, including neurophysiological literature demonstrating the impossibility of making decisions without emotional processes (Damasio 1994; Evans 2002; Hoggett and Thompson 2002; Pyne, 2006).

There is also increasing preference for including and prioritizing holistic concepts in approaches to understanding decision-making. Concepts such as "the intelligence of emotions" (Nussbaum, 2001), "moral capabilities" (van Staveren, 2001), "the "virtues of the mind" (Zagzebski, 1996) and "maternal thinking" (Ruddick, 1989, 2009) are replacing concepts that dichotomize 'reason' and 'emotion' by placing the emphasis on the need to understand the mutually interdependent relationship that exists between intellectual and emotional processes, where the ultimate value is balance.

\subsection{Bridging the Gap - Concluding Remarks}

The critical cartography literature is full of conceptual transformational thinking. Being on the edge of a novel epistemological and ontological stance with respect to maps and mapping, many of the discussions in this literature are either lacking in exemplary detail or are calling on others to begin mapping from this new epistemological and ontological perspective. The Lake Huron Treaty 
participates in this transformation through the iterative development of geonarratives that integrate critical academic and Anishinaabe perspectives, thus providing an example of the new approaches to cartography envisioned in the critical cartography literature. The emphasis on performance and the processes involved in the ontogenesis of the atlas project are linked to the emphasis on action in Anishinaabemowin (the Anishinaabe verb-based language) (Joe Laford, Anishinaabe Elder, personal communication, December 2012).

Critical academic and Anishinaabe perspectives work together in the design and development of the Atlas, exemplifying a two-pronged approach (Johnson, Louis and Pramono, 2006) that involves intersecting knowledges. This is important for overcoming the incommensurability issue referred to by Turnbull (2007), who recommends that cartography be engaged in bringing together incommensurable knowledges. Despite this, significant commonalities have been revealed between critical academic perspectives and Anishinaabe understandings in the process of applying them to the design and development of the Lake Huron Treaty Atlas. Although these perspectives come from contrasting worldviews to some extent, critical academic perspectives have nevertheless begun to question the basic assumptions of the western worldview. These are the very assumptions that form the basis of intercultural incommensurability in the first place; and, once they are replaced with assumptions that are more in tune with Indigenous ways, the world should be a brighter place. 


\section{Atlas Development Reflecting the Past, Reconciling the Future}

In my role as geonarrative and content editor for the Lake Huron Treaty Atlas, I have adopted a similar approach to Jay Johnson, Renee Pualani Louis and Albertus Hadi Pramono (2006), who have allowed the Hawaiian practice of looking backwards to face the future to guide their work:

The concepts of 'past' and 'future' are explained by Hawaiians using bodily directions, the front of the body faces the 'past' while the back faces 'future'. Hawaiians 'face' their 'future' with their backs because the future is an unknown. On the other hand, 'past' is knowable; it can be 'seen' in front of each of us, shaping our character and consciousness. Hawaiians believe that knowing who they are, genealogically, and where they came from, geographically and metaphysically, makes them capable of making more informed decisions about the direction to move in the future. By allowing this concept to guide our work we are focusing our attention first on Western cartography's history, including its role in creating and perpetuating European colonialism (Harley, 1992, 532) and second on the history of Indigenous cartographies (82).

The ongoing collaborative project to create the Lake Huron Treaty Atlas

'looks in all directions', spatializing history by creating geonarratives that:

1. participate in the critical cartographic movement;

2. assume a relational approach to space that emphasizes the performative;

3. are multidimensional;

4. give rise to emergent knowledge (i.e. are non-teleological);

5. bring together past and present;

6. emphasize context;

7. involve a holistic view of development; and

8. make knowledge accessible for people in a way that allows for personal discovery.

The cartographic dimension of the Lake Huron Treaty Atlas alone contributes to its ability to spatialize history by sheer virtue of its concern with where events occurred. The critical cartographic approach contributes further by dissolving the binary between object and action, between maps as objects and 
practices related to the making, use and interpretation of maps. In this vein, viewing the Atlas as a performance provides a further way to spatialize history. One example of this is the way the stories describing the making of the Atlas are integral to understanding the stories presented in the various maps. Together, these stories comprise the overall geonarrative, which includes the online Atlas format and extends to people's conversations and memories of interactions concerning aspects of the atlas project, conference presentations, journal articles and book chapters and other written material (see Appendixes B and E).

The Lake Huron Treaty Atlas is engaged in development along many dimensions through a variety of cybercartographic mapping practices. Cybercartography itself is rooted in Fraser Taylor's early work in Kenya, during which time a 'development from within' approach emerged. The approach I have taken to atlas 'development' has been a similar, get your feet in the mud, transformative approach (Carmen, 2000), which emphasizes non-hierarchical, holistic relationships; and, involves meeting people in their knowledge spaces, finding common ground and participating in emergent mapping practices. These practices in turn feed back into the cybercartographic atlas framework, reinforcing the mutually interdependent relationship that exists between the framework and the Lake Huron Treaty Atlas.

Considering the Atlas project from a capability approach perspective of development (Sen, 1999), it is a capabilities-rich enterprise in a variety of respects. For example, diverse knowledge bases (capabilities or potential functionings) are drawn from in order design and develop (functionings) the Atlas maps. While the Atlas maps themselves are incomplete, some being mere sketches of cartographic possibility (capabilities). There remains a volume of media and 
narrative description to add to the Atlas maps (capabilities). However, their inclusion is in part awaiting the completion of the conversion of the Atlas in phase III to a new software framework, which will have more options for people to interact with maps and contribute to their creation (functionings).

There are two more reasons for waiting to upload new media and narrative description to the Atlas. First, similar to the capability and cybercartographic approaches, the approach to designing and developing the Lake Huron Treaty Atlas resists over-specifying the Atlas maps, their content and direction. The intention for the Lake Huron Treaty Atlas, as part of my thesis project, was that it serve as an invitation to others to participate further in a variety of mapping practices (Kitchin and Dodge, 2007), including uploading media and narrative descriptions, adding critical commentaries to map entries (Brauen et al. 2011), and in some cases making maps of their own.

The cybercartographic framework makes mapping practices possible that are both responsive to and part of reconciliation processes along a variety of dimensions. A good start has been made with regard to the historical research aspect of the atlas project, but a great deal of research remains to be conducted and integrated into the Atlas. Drawing on the thoughts of the late Blaine Belleau Senior from Garden River Nation, one of the first contributors to the Atlas and a descendant of Gimaah Shingwaukonse: Decolonizing history is like "peeling an onion". There are layers upon layers of broken relationships extending through time and across space that need healing. At the same time, there have been some healthy intercultural relationships sprinkled throughout time, whose inclusion could enhance this decolonization process. 
Over the past seven years, a growing sense of community familiarity has developed in relation to the Lake Huron Treaty Atlas, the expertise of the research team and the resources of the Geomatics and Cartographic Research Centre. This development reflects the importance of longevity and continuity in a participatory atlas project such as the Lake Huron Treaty Atlas, which is becoming an effective knowledge dissemination tool through the active participation of a diverse array of contributors.

In this thesis, I have viewed the incommensurability barrier as a permeable barrier. I have been thinking about David Turnbull's (2007) statement regarding the "dialogical tension" that exists between worldviews for some time. Being of a bridging mentality, while I agree with Turnbull regarding the existence of incommensurabilities, at the same time, I have encountered many individuals (either in person or via the writing in their papers) who are breaking chinks in that armor. Events such as the International Forum on Indigenous Mapping for Indigenous Advocacy and Empowerment, held in 2004 in Vancouver, British Columbia provide an example of bringing together "Indigenous peoples engaged in mapping, as well as individuals who assist them in these efforts" (the International Forum on Indigenous Mapping, 2004).

\subsection{Future Implementation: Potential and Challenges}

Issues related to the politics of cyberspace (Crampton, 2003), including privacy, uneven distribution of resources, (cognitive) accessibility, and epistemological and ontological commensurability, are always close on the horizon, and solutions to cognitive accessibility are currently being pursued in technological research, but could also involve training individuals in varying degrees of technical expertise. 
A related issue is the politics of technology, which Eddie Benton Banai (1988) comments on in his telling of the Seven Fires Prophecy. Banai is not alone in casting technology in a negative light. It is true that there are many negative aspects associated with technology; but if it is brought into balance with "life", it may not be such a bad thing after all. There may seem to be a paradox in stating this work is part of the Seven Fires Prophecy in terms of finding our way back, while at the same time it makes use of and works with the 'technology'. The drawbacks associated with technology are well known, and virtually all of them can be mitigated for, including more environmentally sustainable ways of powering and creating technology and appropriate protocols for "healthy" technology use. As Thrift (2004a and b) has pointed out, and as the Anishinaabe language carries inherently, material "objects" can be animate entities, and we exist in relationship to them. Elder Jacob Wawatie's simple yet profound teaching that the entire world we have constructed around us comes ultimately from Mother Earth. Thinking about my aging car, which has been my trusty steed on many a ride through the Treaty region, I have reflected on how much of it is transformed dinosaur bones and sand.

\subsection{Turtle Speed}

Helga Nowotny (2009) describes a new transdisciplinary form of knowledge production that highlights sharing across knowledge spaces. In addition to reminding us this approach to research involves patience, Nowotny discusses the benefits of new two-way communications between science and society, and answers the question concerning the quality of this new knowledge production by recommending robustness as the standard by which it should be assessed. The atlas project has aimed at producing this sort of robust knowledge. 
Patience is a virtue in transdisciplinary research. Part of reason for taking time in this project has been the need - and the desire - to get to know the people and the land - to spend time - and to get to know myself in the process. A related reason is the need to the time it takes to integrate knowledge from many different knowledge spaces into geonarratives, since this ultimately involves collaborating with a diverse range of contributors from a variety of intersecting knowledge spaces.

Reflecting on a conversation I had with Elder Gordon Waindubence from Sheguiandah First Nation on Mnidoo Mnising in the early stages of putting this thesis draft together, Gordon talked about how he is from the Turtle Clan and often takes a good long time to reflect and come to a determination. Considering the many dimensions of the Lake Huron Treaty relationship process over time and across space, and the many working maps that have been created to begin to reflect these dimensions, it has been necessary to take the turtle's pace in many respects. In some cases, further development of mapping ideas has had to wait until the technological developments are in place to implement them; in other cases, further time for conceptual and other development has been necessary.

\subsection{Shifting the Research Focus}

As I was going through the process of completing the ethics forms at the beginning of phase II in the spring of 2009, I realized the extent to which the atlas project did not conform to the standard idea of research. The most significant contrast concerned the lack of research subjects in this project, and therefore, the failure of the project to study them. In shifting the focus to the production of a cybercartographic atlas product based on the incorporation of multiple perspectives, both in the production process and in the resulting content 
presentation, this project emphasizes the roles of individuals as atlas contributors, engaged in various collaborative mapping activities, instead of being research 'subjects'.

Moreover, throughout the knowledge-making process, "community" has been construed broadly to include all interested people who have become involved in one way or another in the project and who have been sympathetic to a non-dominatory approach to knowledge construction, including Lake Huron Anishinaabek, academics, technical specialists, and people at the grassroots level. These collaborative efforts have been deeply relational, in that they have involved the emergence of a new network of "investigative colleagues," in contrast to the more traditional "researcher/researched" paradigm of investigation. Moreover, the investigations have been relational in nature, in that they have centered on the goal of coming to a better understanding of the evolving historical geography of Lake Huron Treaty-based relationships instead of "studying" one particular group of people or the other as research subjects (Caquard et al., 2009, 88).

\subsection{Benefits of the Cybercartographic Atlas Framework for Research}

Conducting my $\mathrm{PhD}$ research within the cybercartographic atlas framework gave me freedom to do field research over an extended amount of time that continues to this day, and to integrate this practice with academic writing and the preparation of presentations. Working with framework has also allowed me to take a holistic approach to research. An example of this is the way the thesis and the atlas are holistically interrelated. In part IV, I use "the map" to guide the narrative structure of the storytelling. As they stand, the maps are just sketches and are incomplete in terms of scope and content. In writing the 
narrative chapters (and the thesis more broadly), I have created new content, which can in turn be added to the maps.

Taylor and Pyne (2010) emphasize how intuition and practice preceded the theoretical development of cybercartography. While this has also been the case with the development of the Atlas, it must be remembered that neither Fraser Taylor nor I were a complete stranger to the world of theory at the time we embarked on our respective projects. This emphasis on the importance of intuition and practice has made the cybercartographic atlas framework an ideal match for the emergent, two-pronged approach practiced in the Lake Huron Treaty Atlas.

\subsection{The Lake Huron Treaty Atlas and Development}

In implementing a holistic approach to development, the atlas project could function as a model of development similar to that presented by Sara Ruddick in Maternal Thinking: A Politics of Peace (1989), in which she suggests that the holistic discipline of maternal rationality could be a model for development.

In addition to the manner in which taking a holistic approach to development has given rise to emergent knowledge, 'personal' development has been effected as well. For example, between January and June 2010, a high school student who was experiencing difficulties succeeding in the school system became a co-op student with the atlas project. In addition to having a rich and challenging learning experience, the student arrived at a new awareness of Anishinaabe worldviews and contributed to the project in a way that reflected his skills and interests. He also earned the final credits he needed to graduate with honours, and gained the self-esteem and direction to proceed to college in a program related to his co-op assignment. The following text from this student's 
final report provides an indication of the positive impacts of the co-op component of the atlas project, in addition to providing a glimpse into some details relevant to the making of the Atlas, including the Travels in the Making of the Atlas Map:

The last few months I've had the opportunity to gain knowledge and experience working in the post secondary research environment. I've carried out my co-op term at the (Geomatics and Cartographic Research Centre) GCRC at Carleton University. I was encouraged to use the skills I had previously obtained through my high school studies as well as those I have learned working at GCRC to aid in the development of a cybercartographic (online interactive multimedia) atlas. This co-op assignment has been an exceptional experience and I have learned quite a bit about the processes involved in creating cybercartographic atlases.

Overall I was amazed at how much I was able to contribute as a co-op student. In the first few weeks I started out with simpler jobs, such as transcribing surveyor J.S. Dennis' Field notes and Diaries, which were written in the years following the signing of the Robinson Treaties in 1850. I then had the opportunity to work with multimedia making a slide show with some pictures I had taken the previous summer when I was in the field. Creating the slide show also involved some photo editing, a project that allowed me the freedom to use my own judgment and creativity. The level of freedom I had to preform these tasks made it very enjoyable.

When I began to get used to working with atlas content, I was given the data to populate several interactive maps, for example, Season 2 Leg 2 of the atlas. I was able to create points using coordinates from each of the surveyor's stops. Each stop needed to have the corresponding diary entries, audio files, image files and proper information inputted. I was able to input all the data and attach all the media myself. It was rewarding seeing it all come together and beginning to understand the concepts of the interactive atlas and how it is much more than a mere map.

After uploading and creating the initial season, we had to make some revisions. Many of the audio files weren't split up for each day (each diary entry). So I used audio editing tools to split the files appropriately then I re-uploaded them. The issues weren't just with the audio; some of the pictures had improper formats or files names so they would not appear on the site. I investigated into the individual error for each file and corrected it. A few formatting problems remained so we met with Glenn to discuss them and see what he was able to do.

Aside from working directly with the atlas a lot of research was involved with the project. Since community involvement is a large focus, the idea of getting schools involved seems only fit. I 
researched Ontario schools and found schools in the towns near reserves and schools that had Native Studies courses and made up a report with contact information. I also learned a lot from my research into Documentary Making, Interactive Maps and Mapping in the Digital Age and also about the general Native Treaty history in Northern Ontario.

Later into the term I was able to go on a weeklong field trip, which also gave me the opportunity to learn about travel planning, and which I was able to do quite a bit of. We met with some different people and did a lot of promoting the atlas and promoting community involvement. Our first stop was Toronto where we met a man named Isadore Toulouse and his technical assistant Nathan Big Canoe, who have started a weekly online Ojibwe language class. We then continued up to Manitoulin Island where we met a man named Joseph Laford, who had a lot of knowledge and a deep perspective of Anishinaabe culture and society. When we finally made it to Sault Ste. Marie we visited Garden River First Nation where we spent two days with a Band Counsellor named Blaine Belleau Sr., who had a lot to say about the history and politics of treaty-based relationships. Along with our meetings, some of which were filmed and recorded, we took photos and used a GPS device to track our route. The trip proved to be beneficial and it was also a really engaging learning experience for me.

The weeks following the trip I had to organize all the media and information we collected in the field. We were given some notes on Treaty history and Native history in Ontario so I transcribed those. I continued on to edit the pictures, videos, images of documents and maps. I organized everything from our trip and made sure the video files were backed up on a few hard drives. Now it's my last week and I'm going to attempt to create a logo for the GCRC. Overall I really enjoyed my placement and would highly suggest it to other co-op students who are interested (Jesse Young, June 16, 2010).

This development model through mapping practices has been extended in

phase 3 to another important mapping project in the work that has begun to involve a Gitigaan-ziibing (Garden River) Adults in Motion co-op student in the eventual creation of the Garden River Natahwegidjig Mahjeeshkahwaun Map, based on the Garden River Natahwegidjig Mahjeeshkahwaun "Adults in Motion" Archives.

11.6 The Lake Huron Treaty Atlas and the Seven Fires Prophecy-A Map Proposal 
In the spring of 2010, I began to think about the twin prophecies of the fourth fire, the fire of two possibilities. This thinking led to the emergence of the idea for the Tracings map, which would use the Seven Fires Prophecy as the interpretive framework or background for understanding the genealogy of western thought, perspectives and assumptions: Turning the tables, so to speak. This map idea was based on an insight I had gleaned from my reflections on Elder Commanda's reading of the belt after the launch of the Treaties Module in April 2008: The seeds for the positive possibility, which has not yet come to fruition, were latent in the newcomer, perhaps from the Beginning. David Turnbull (2000) supports this thinking when he maintains that Western technoscientific knowledge does not rest on a purely neutral and objective basis; and that further, it is only one of many knowledge systems. According to Turnbull, "technoscientific knowledge, upon which the concept of modernity is based, epitomizes planning, rationality and order ... Rather than being governed by logic and method, modernity's drive for order conceals its messy, contingent, unplanned and arational character" $(2000,1)$. The brief account given in chapter 7 of Turnbull's performative "talk, template and tradition" analysis of the multigenerational collaborative project to construct the Chartres cathedral illustrates this as well.

If we look at some aspects of the evolution of the Western worldview and thought in the context of the fourth fire prophecies delivered by the two twins, we can see that the two possibilities were latent in the West. Indeed, in the writings of many notable Western thinkers lie two paths. For the purposes of the initial map design, the genealogy extends as far back as the Ancient Greeks, although, it is important to acknowledge that they too "came from" somewhere. 
It begins with a basic split in ethics of Plato and Aristotle. Whereas Aristotle's ethics was contextualist and particularlistic, emphasizing the need to find balance between emotions, thought and actions, Plato's ethics tended toward universalism, privileging thought over emotions, which were primarily seen to interfere with thought and promoting the idea that rules or principles could be derived to "govern" thought and action. Recognizing the breadth of the philosophies of these two individuals, this contrast between the two thinkers is intended to illustrate two threads of thought that carried on into the future and influenced Western thought in profound ways. Both thinkers were no doubt influenced by the worldviews of the societies in which they lived, and in turn influenced those societies, and the assumptions that underpinned their perspectives carried forward to influence others in the "Western tradition" through time and across space.

Further thinkers to be included in the Tracings Interactive Map include individuals such as Gerald of Cambrensis (1147-1220), whose writings on "civilizing the Irish" in the twelfth century were drawn upon for the following five hundred years in further imperial efforts to "civilize" others (McVeigh and Rolston, 2009); Francis Bacon (1561-1626), a key figure in both the "scientific revolution" and colonial thought whose quest to establish a material science based on neutral and objective observation overshadowed the ways in which his spirituality and emotionality contributed to that quest (Irving 2006; Varvis 1983); Rene Descartes (1596-1650), the "father of dualism who was nevertheless inspired to his "I think therefore I am" epiphany by a dream (Withers 2008); John Locke (1632-1704), whose thoughts on liberty and freedom were skewed by the development of his thinking on individual property rights (Armitage 2004; Farr 
2008); and, J.S. Mill (1806-1873) who, similar to Locke, had a direct effect on colonial policy, and whose writings on settler colonialism in his later life reflected a disdain for "colonial violence" and a growing "melancholia" with respect to the manner in which colonial projects were being implemented (Bell 2010, 31) . These thinkers both influenced and were influenced by the societies in which they lived, and had both direct and indirect effects on government policies, including colonial policy. Although all of these thinkers played a role in the evolution of the "Western worldview", aspects of their thought belied what Turnbull has referred to as an 'arational' nature, which is not to be confused with “irrational".

It is important to draw attention to the two possibilities spoken of by the prophets at the fourth fire, the idea being that the potential for either intercultural friendship or intercultural "foeship" was linked to worldviews that grew and changed over time. The seeds for both possibilities had been planted in the West. However, one of the seeds was watered more than the other resulting in Western "culture" that was predominantly Eurocentric and imperialistic, in addition to becoming increasingly fragmented with respect to fundamental epistemological and ontological assumptions that privileged binary opposition and dualism rather than holism.

Although it may seem that including a map such as the Tracings Interactive Map in an atlas designed to tell the story of the Lake Huron Treaty Relationship Process is a little far afield, a broad multidimensional approach is necessary, one that extends to the scale of worldview and seeks to understand the roots of its genealogy, in order to contribute to the removal of barriers related to misunderstandings that have resulted in colonial exploitation not only in the 
particular case of Lake Huron Treaty-based relationships, but extending beyond as well. In order to accomplish this goal, it is important to overcome challenges associated with misappropriation of Indigenous knowledge and related challenges associated with incommensurability of knowledges in the design and development of the atlas, and in the Tracings Interactive Map, in particular. Presenting the Seven Fires Prophecy as the interpretative framework for understanding one possible thread in the genealogy of the worldview underlying colonialism, and inviting Anishinaabe input in the design and development process, is one way to begin to do this, and to depart from centuries of misappropriation and exploitation of Indigenous knowledges in a colonial context (Belyea, 1992; Jolly 2007).

An important aspect of this atlas work in general is its concern with intercultural relationships where it does not study one culture or another ethnographically, but engages with all (Caquard et al., 2009). It avoids the criticism often directed at efforts to apply geospatial technologies in the presentation of Indigenous knowledges, and phenomena extending to experience, by emphasizing the importance of trails and journeys rather than particular points on a map; and it follows Turnbull (2007) and others referred to in this paper in "rethinking knowing and mapping - where the key questions relate to the similarities and differences in the ways space, time and movement are performed and to how those similarities and differences are handled" (Turnbull, 2007, 141). In the work to design and develop the Tracings Interactive Map, and in the atlas work more broadly, mapping is repurposed: Instead of providing reinforcement for colonial or other hegemonic forms of domination by one group of people and institutions over others, the purpose of this mapping is 
to track the development of knowledge over time and space, in an effort to contribute to a new space of mutual understanding (Turnbull 2007).

\subsection{Energy of the Dance and Performativity - Concluding Remarks}

A performative or processual approach to mapping and atlas making is central to this project (Caquard et al., 2009). For example, the process of writing this thesis goes beyond the thesis itself and is part of the development of the Atlas. In my June 2013 conversation with Gordon Waindubence he told me that many students write theses and then disappear: No one ever gets to see what they wrote. When he asked me what I was going to do with the Atlas, I replied immediately, "I'm going to put it in the Atlas". And indeed I will. Of course, I will find a way to incorporate the elaborations, reflections and commentaries in a way that works with the geonarrative construction in doing so. Practicing my Anishinaabe Grandmother's wisdom of not wasting anything, which she passed down to me over many visits and many stories of the past, the reflexive and iterative nature of the Atlas development process allows me the flexibility to work with others to create unfinished geonarratives, to mix and blend, to share and learn, to be open to possibilities, including inviting the Ancestors to be a part of the process: To bring them into heart and memory in a way that allows them to guide and share in the process.

The energy of this process is similar to what I have witnessed in the energy of the hoop dance. Blain Commanda from Serpent River First Nation told me a story in June 2013 about the hoop dance. The dancer is the loon spirit and the dance is the story of creation in which the loon reproduces creation by combining and re-combining her many hoops. Lisa Odjig from Wausauksing First Nation in the Lake Huron Treaty region is a world champion hoop dancer 
who has performed the hoop dance many times. ${ }^{30}$ Similar to this dance, my part in the Lake Huron Treaty Atlas geonarrative design and development involves reflecting the multiple dimensions of social and cultural, political and economic 'reality' that we have created for ourselves; it involves proceeding in a circular or holistic fashion; it takes time and involves stamina, strength and coordination to balance multiple objects through interlinking them. And the result through this process is energy: the energy of the dance, propelled by the drum, the heartbeat.

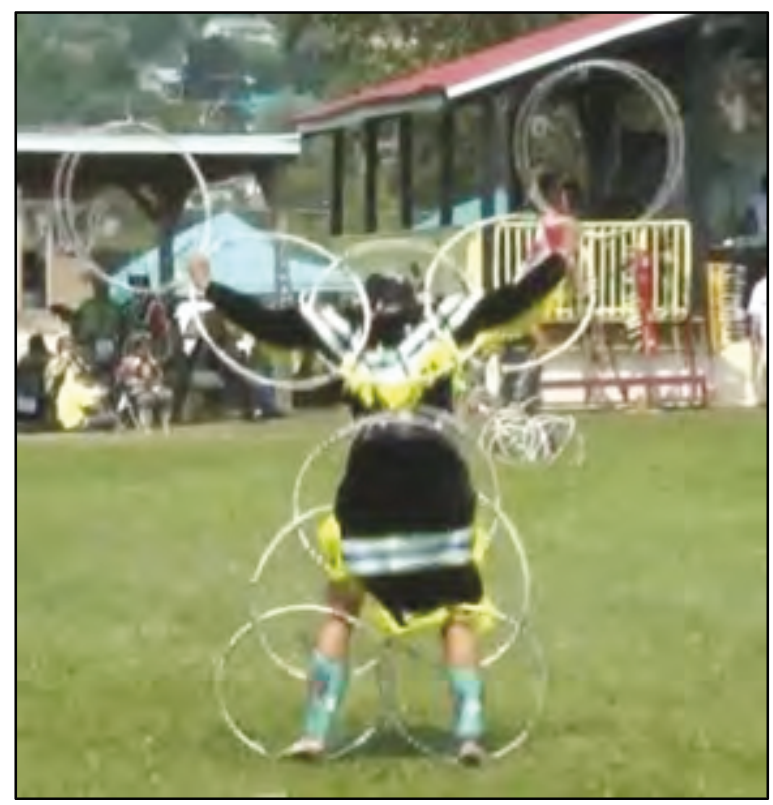

Figure 11.1. Lisa Odjig performing the hoop dance at the Wikwemikong First Nation 2012 Powow.

The atlas development process involves a variety of interpersonal interactions, knowledge exchanges and actions related to the Lake Huron Treaty "story" and to atlas design, development and use. The line between the Atlas as a material object and the design and development processes involved in its making is blurred; the dichotomy is diminished. Insofar as the Atlas is being designed to

30 I first saw Lisa Odjig performing the hoop dance at the Banff Centre in 2004. See http:/ / www.youtube.com/watch?v=TwBtOsdWsyI for a video with Lisa Odjig hoop dancing at the Wikwemiking Unceded First Nation 2012 Powwow. 
allow for ongoing critical input and contributions, the map user can also become the mapmaker. In this respect, designing, developing and using the atlas are all intertwined, and work is progressing to design the digital architecture that will allow for critical comments to be overlain on maps such as the Tracings Interactive Map (Brauen et al., 2011).

The specific purposes for cartographically interpreting the Western worldview from an Anishinaabe perspective by incorporating Anishinaabe ways into the making of the Atlas are related to (1) the broader purposes of the Atlas, (2) the purposes of critical cartography and Indigenous methodologies, and (3) the purposes of the Seven Fires Prophecy itself. These purposes in turn relate to the reasons the tables need to be turned: not only to respect and revitalize Indigenous knowledges, not only to contribute to increased awareness of unique knowledges in an effort to enhance mutual understanding, but also to acknowledge the significance of the traditional Anishinaabe perspective; an approach that is grounded in a broad and inclusive holistic cosmology: "It is a holism that goes beyond the empirically based concept of a unified physical universe ... [and] that incorporates the unity of spiritual and physical worlds" (Louis 2007, 133-4) in a way that aims for balance as an end in understanding and relationships. In contrast, the hegemonic cosmology of the West, including the scientific revolution and colonialism, has tended to compartmentalize the unity envisioned by Indigenous peoples in a way that aims for prediction and control as an end in understanding and relationships (Rostow, 1971; Johnston and Murton, 2007). It is important to resist the tendency to conceive of the distinction between Indigenous and Western cosmologies as a binary opposition. The thought, dialogue and writing of most of the authors referenced in this thesis, 
and the thesis itself, represent a growing undercurrent of change in the conventional flow of Western hegemony, providing a glimmer of hope that true bridge building between cultural worldviews is achievable. 


\section{Afterword: Initial Reflections on the Thesis-writing Process}

I have been working on the writing toward this thesis for many years, and have produced a variety of authored 'outputs'. However, it was not until June 21, 2013 that I finally settled on my thesis template and approach. Not long before, I had decided to 'go out on a limb' and take an emergent approach to the dissertation preparation process.

It has often occurred to me since the time I decided to have the Rainbow Raven and the Fly in the Bottle Story illustrated, that this story is not only the spirit and intent - the aim - of the atlas project; it is also the method. At least, it is the method I have taken as the geonarrative and content editor. Although it is not written in the story at the beginning of this thesis, the raven first flies in wide circles, high in the sky, surveying a broad territory; she picks up shiny objects and brings them back to her next; she sorts them, arranges them and otherwise examines them; then she selects; and, flying around again, and again and again, she finally settles on the placement of the object, with the purpose of showing the fly the way out of the bottle. This has been my experience in the preparation of this thesis, which has been the same sort of experience I have had as the geonarrative and content editor for the Lake Huron Treaty Atlas. Following the circle in this way during the thesis writing process, I was never entirely sure what was going to emerge. My talks with spiritual guide and Elder Joe Laford during this process, and his gift of a 'hoop dancer' eagle feather, confirmed my own feelings that I had all the pieces I needed to put the thesis together. And in reality, it was true. The challenge was that I had more than enough after seven 
years of working on the project, with much prior writing and thought to draw on.

There are many stories that remain to be told about my experiences working on the atlas project. In writing this thesis, I have had to leave many stories, ideas, insights, quandaries and qualifications aside. I have resisted on many occasions the temptation to get into the details of Lake Huron Treaty historical geography, in part because it is covered to some extent in previous writing and because it is covered - and will continue to be covered cartographically in the Atlas itself. As far as future writing goes, I plan to continue on this reflexive journey by telling the story behind the story in more detail. For example, this afterword provides only the merest of sketches to illustrate the extent to which I incorporated ceremony and "talk" with people of all walks of life, including Elders and youth, in my thesis-writing quest. The fuller story remains to be told.

In the process of writing this thesis, I have had the chance to review and reflect on much media, and was fortunate to have my brother, Jason Pyne, transcribe a large portion of Anishinaabe wisdom reflected in it, including the quoted text below. As a prelude to what lies ahead in terms of incorporating the Anishinaabe perspective in the making of the Atlas of the Lake Huron Treaty, I end with the words of Elder Lewis Debassige from M'Chigeeng First Nation at the May 2012 opening ceremony for the Atlas, which was officially opened with a prayer and a welcome to Algonquin Territory by Elder Jo Wawatie (see Appendix D for video clip). Since I first met Lewis in February 2010, he has kindly offered his time on many occasions to share his knowledge and 
participate in engaging conversations that have both enriched my perspective and allowed me to express it more clearly:

Bringing together what she has found, and gathering, and preparing it in such a way that the next generation will taste that past, and enjoy savoring the stories of the relevance of place names, and what happened there. Even from difficult history, like the Residential Schools Experience come lessons that will help us now and people who will, soon enough, step in our shoes. That was one of my Grandfather's Teachings. He says that sorrow, that difficulty, that great trial that we go through, only tempers our spirit so we can better meet the future, and for several generations thereafter.

So five years to rebuild this collection of knowledge from the past is not a long time. And I reminded Stephanie that it has taken Government-sponsored Institutions like the Church, the Indian Agents and the System, decades and centuries to disassociate ourselves or disinherit us from our past. And it's going to take a good long time to recover from that. And that's the work that's being done by our students and our young people in institutions like Carleton. And I'm glad. I mean, I've been involved with Alan who's dragged me to sessions over here. I feel enriched by that experience, having to share my off-the-wall stories at times with scholars and academics.

The thing is, we all have our own path - as Joe said in his words that opened this gathering - a path of our own. And I want to share a story with you that I heard from Dan Pine, years ago. I was doing pretty much the same thing as Stephanie is doing: gathering stories and the relevance that they might have today.

He told of an aging Elder that lived at the foot of a mountain. He was well respected. He was an accomplished herbalist and healer, and people who were sick would come from a great distance to visit him so that they could be favoured with his treatment. And then he let it be known that he was getting up in years so he needed a young helper who would help continue his practice that he had established. So, many came. Many young men came. But one day there was a particularly interesting young man, thirteen years old- very robust, obviously athletic - and eager - who showed up first.

He said to the Elder, "I've been sent by my Father to come and learn from you." He says, "I learn fast. I listen well. And I'll do any chores or hunting or fishing for you, so that we can spend more time together, in this supposed great exchange that is supposed to happen".

So the old man said, "Okay, meanwhile, you can go and cut my wood and prepare a meal".

And in comes this second one. Not as athletic, not as robust: kind of reserved, with his head down.

And he too, says, "I was sent by my Father to learn from you. But obviously my competition is a lot better suited than I am". So he says, "Dismiss me now so I can go back and tell the disappointing news to my father."

The old man replied, "No, you stay here with us because I have a task for both of you to do." So they had a meal and that afternoon he said, "I want both of you to climb that mountain. And it takes a healthy man half a day to climb, and get back. So you should, as men-to-be, spend the night on top of the mountain, 
and you'll see the big Pine trees. And on top of the Pine trees are Eagles nests. I want you to bring an eaglet's down as proof that you reached the nests."

So, off they went. Evening fell. Next morning came.

Sure enough, the athletic young boy returned first. He told the old man, he says, "Look, I reached the Eagles' nest. Here's the eaglet's down, and I could have gotten back here before nightfall! But I decided to follow your advice and spend the night on the mountain."

And the Elder asks, "So, what did you see?"

"Well, it's a majestic sight on top of the mountain. And the Eagles spoke to me", he says. "They made me look; and on the other side, there are other mountains. It made me wonder who lives there. And what medicine, what knowledge they have to share with us? And I'd like to go and see, and visit those people that live there."

So the Elder accepted this. He says, "That you must do. That is your calling, to visit those people, learn from them, and bring back those good things from them".

So, just before noon the less-athletic kid wanders in, with his head down, and he says, "I didn't even get to half-way up the mountain. I wandered, meandered and followed the stream; as a matter of fact I came back down to the stream." He says, "In fact, I was entertained by the birds, and the bugs, and insects. And wondered, what medicine they have? And what knowledge of flora they could teach me? Underneath my very feet a miracle is happening! And I still wonder about that."

And the Elder says, "You shall be my student."

And the young man was quite surprised, and says, "How is that?"

And the Elder said, "Seventy years ago I came to visit an Elder that lived here who was very accomplished in healing. And this is how far I got. I never got to the top of the mountain."

So the lesson there is each of us have our purpose. Some of us have to stay right here and discover what is beneath our feet, some of us have to reach out. That's my story for you, on this occasion. Miigwetch Stephanie Pyne.

Aho. 


\section{Bibliography}

Akerman, J. (1993). From Books with Maps to Books as Maps: The Editor in the Creation of the Atlas Idea. In Editing Early and Historical Atlases: Papers Given at the Twenty-ninth Annual Conference on Editorial Problems, University of Toronto, November 5-6.

Akerman, D. (1991). On the Shoulders of a Titan: Viewing the World of the Past in Atlas Structure. Unpublished PhD Dissertation, Pennsylvania State University.

Aitken, S. C. (2002). Public Participation, Technological Discourses and the Scale of GIS. In W. Craig, T. Harris and D. Weiner (Eds.), Community Participation and Geographic Information Systems. London: Taylor \& Francis, 357-66.

Aitken, S. C. and Michel, S.M. (1995). Who Contrives the "Real" in GIS? Geographic Information, Planning and Critical Theory. Cartography and Geographic Information Systems, 22(1), 17-29.

Alkire, S. (2002). Dimensions of Human Development. World Development, 30(2), 181-205.

Allen, J. (2004). The Whereabouts of Power: Politics, Government and Space. Geografiska Annaler, 86B, 19-32.

Allen, W.A. (2010). Anishinaabemowin: Traditional Language in the Naming of Archaeological Sites. Arch Notes, 15(1), 5-10.

Allen, W.A. (2006). Akikpautik in the Reconciliation of Worldviews about Archaeological Research. Presentation at Canadian Archaeological Association Conference, Toronto, Ontario, May 26.

Allen, W.A. (2002). No Longer a Terra Incognita. Paper presented at the 35th Annual Meeting of the Canadian Archaeological Association, Ottawa.

Allen, W.A., L.M. Brady, and P. Decontie. 2008. Manaadjiyindj iyaa manidoo nayaagadjitoodj kije-asin mazinaakobiihiganan - Honouring the Spirits of Sacred Pictographs. Pp. 277-289, in C. Dignard, K. Helwig, J. Mason, K. Nanowin, and T. Stone (Eds.). Preserving Aboriginal Heritage: Technical and Traditional Approaches, Proceedings of Symposium 2007. Canadian Conservation Institute, Ottawa, Canada.

Alvesson, M. and Sköldberg, K. (2000). Reflexive Methodology: New Vistas for Qualitative Research Towards a Reflexive Methodology. London: Sage.

Amin, A. (2004). Regions Abound: Towards a New Politics of Place. Geografiska Annaler, 86B, 33-44. 
Archibald, J. (Q'um Q'um Xiiem) (2008). Indigenous Storywork: Educating the Heart, Mind, Body, and Spirit. Vancouver: UBC Press.

Armitage, D. (2004). John Locke, Carolina, and the Two Treatises of Government. Political Theory, 32(5), 602-627.

Arnold, K. (2002). Anti-epiphany and the Jungian Manikin: Toward a Theory of Prepsychotic Perceptual Alterations. Journal of Phenomenological Psychology, 33(2), 245-275.

Ashmore, M. (1989). The Reflexive Thesis: Wrighting a Sociology of Scientific Knowledge. Chicago: University of Chicago Press.

Asmal, K., Asmal, L. and Suresh Roberts, R. (1996). Reconciliation through Truth: A Reckoning of Apartheid's Criminal Governance. Cape Town: David Philip.

Baldwin, D., Daubs, M., Tomasino, J. (2004). Infusing Traditional Storytelling and Design Techniques into Multimedia Interface Design, International Conference on Education and Information Systems: Technologies and Applications, Volume 1, Proceedings, 308-312.

Barnes, T. and Duncan, J. (1992). (Eds) Writing Worlds: Discourse, Text and Metaphor in the Representation of Landscape. London: Routledge.

Barreiro, José. (2004). Indigenous Geography As Discipline Arrives. Indian County Today, Available at http:/ / indiancountrytodaymedianetwork.com/ictarchives/2004/03/31/indigen ous-geography-as-discipline-arrives-93411

Belfy, P. (2011). Three Fires Unity: The Anishnaabeg of the Lake Huron Borderlands. Lincoln: University of Nebraska Press.

Bell, D. (2010). John Stuart Mill on Colonies. Political Theory, 38(1), 34-64.

Belyea, B. Images of Power: Derrida, Foucault, Harley. Cartographica, 29(2), 1-9.

Ben-Ari1, A. and Enosh, G. (2010). Processes of Reflectivity Knowledge Construction. Qualitative Social Work, 10(2), 152-171.

Benton-Banai, E. (1988). The Mishomis Book: The Voice of the Ojibway. St. Paul, Minn.: Indian Country Press.

Beyersdorff, M. (2007). Covering the Earth: Mapping the Walkabout in Andean Pueblos de Indios. Latin American Research Review, 42(3), 129-160.

Bhandar, B. (2004). Anxious Reconciliation(s): Unsettling Foundations and Spatializing History. Environment and Planning D. Society and Space, 22, 831-845. 
Bodily, C.L. (1994). Ageism and the Deployments of Age: A Constructionist View. In T.R. Sarbin \& J.I. Kitsuse (Eds.), Constructing the Social. London: Sage, 1994, 174-94.

Borges, L. (1935). A Universal History of Infamy. Argentina: Editorial Tor.

Bourdieu, P. and Wacquant, L. (1992). An Invitation to Reflexive Sociology. Chicago: University of Chicago Press.

Borrows, J. (2002). Recovering Canada: The Resurgence of Indigenous Law. Toronto: University of Toronto Press.

Borrows J. (1999). Sovereignty's Alchemy: An Analysis of Delgamuukw v. British Columbia. Osgoode Hall Law Journal, 37(3), 537-596.

Brauen, G., Pyne, S., Hayes, A., Fiset, J.P. and Taylor, D.R.F. (2011). Encouraging Transdisciplinary Participation using an Open Source Cybercartographic Toolkit: The Atlas of the Lake Huron Treaty Relationship Process, Geomatica, 65(1), 27-45.

Brauen, G. (2006). Designing Interactive Sound Maps Using Scalable Vector Graphics, Cartographica, 41(1), 59-71.

Brealey, K. (1995). Mapping Them 'Out': Euro-Canadian Cartography and the Appropriation of the Nuxalk and Ts'ilhqot'in First Nations' Territories, 17931916. The Canadian Geographer. 39(2), 140-156.

Brown, W. (2001). Politics Out of History. Princeton, NJ: Princeton University Press.

Buber, M. (1996). I and Thou. New York: Touchstone.

Burrell, G. \& Morgan, G. (1979). Sociological Paradigms and Organizational Analysis. Aldershot: Gower.

Buttimer, A. (1974). Values in Geography. Washington: Association of American Geographers.

Buttimer A, Seamon D. (Eds). (1980). The Human Experience of Space and Place. London: Croom Helm.

Calás, M. and Smircich, L. (1999). Past Postmodernism? Reflections and Tentative Directions. Academy of Management Review, 24(4), 649-71.

Camerini, J. (1993). Evolution, Biogeography, and Maps: An Early History of Wallace's Line. Isis, 84, 700-27.

Caquard, S. (2011). Cartography 1: Mapping Narrative Cartography, Progress in Human Geography,37(1), 135-144. 
Caquard, S., Pyne, S., Igloliorte, H., Mierins, K., Hayes, A. and Taylor, D.R.F. (2009). A "Living" Atlas for Geospatial Storytelling: The Cybercartographic Atlas of Indigenous Perspectives and Knowledge of the Great Lakes Region, Cartographica, 44(2), 83-100.

Caquard, S. and Taylor, F. (2005), Art, Maps and Cybercartography: Stimulating Reflexivity among Map-Users, in Taylor, D. R. F. (Ed.) Cybercartography: Theory and Practice, Volume 4 in Modern Cartography Series, Amsterdam: Elsevier, chapter 12, 285-307.

Carmen, R. (2000). 'Prima Mangiare Poi Filosofare'. Journal of International Development, 12, 1019-1030.

Carver, S. (2003). The Future of Participatory Approaches using Geographic Information: Developing a Research Agenda for the 21st Century. Urban and Regional Information Systems Association (URISA) Journal, 15, APA I, 61-72.

Carver, S., Evans, A., Kingston, R. and Turton, I. (2001). Public Participation, GIS, and cyberdemocracy: Evaluating on-line Spatial Decision Support Systems. Environment and Planning B, 28(6), 907-21.

Chamberlain, B. (1891). Indian Treaties and Surrenders, from 1680 to 1890. Ottawa.

Chambers, K., Corbett, J., Keller, C., Wood, C. (2004). Indigenous Knowledge, Mapping and GIS: A Diffusion of Innovation Perspective. Cartographica, 39(3), 1932.

Chia, R. (1996). The Problem of Reflexivity in Organizational Research: Towards a Postmodern Science of Organization. Organization, 3(1), 31-59.

Chute, J. (1998). The Legacy of Shingwaukonse: A Century of Native Leadership. Toronto; Buffalo: University of Toronto Press.

Circle of All Nations, (2010). A Report on the Vision for the Asinabka National Indigenous Centre. Community and Protective Services Committee Report, 60, 19 November.

Clandinin, D. J. and Connelly, F. M. (2000). Narrative Inquiry: Experience and Story in Qualitative Research. San Francisco: Jossey-Bass.

Clifford, J. \& Marcus, G. (1986). Writing Culture: The Poetics and Politics of Ethnography. Berkeley: University of California Press.

Connerton, P. (1989). How Societies Remember. New York: Cambridge University Press

Cooper, R. (1990). Organization/Disorganization. In J. Hassard and D. Pym (Eds), The Theory and Philosophy of Organizations. London: Routledge, 167-197. 
Corner, J. (1999.) The Agency of Mapping: Speculation, Critique and Invention. In D. Cosgrove (Ed.), Mappings. London: Reaktion Books, 213-252.

Cosgrove, D. (2008). Geography and Vision: Seeing, Imagining, and Representing the World. London and New York: I. B. Taurus.

Cosgrove, D. (2005). Maps, Mapping, Modernity: Art and Cartography in the Twentieth Century, Imago Mundi, 57(1), 35-54.

Couclelis, H. (2004). The Third Domain: The Spread and Use of GIS within Social Science, Cartographica, 39(1), 17-24.

Couclelis, H. (1996). The Death of Distance. Environment and Planning B: Planning and Design, 23, 387-389.

Cowen M.P. and Shenton, R.W. (1996). Doctrines of Development. London; New York: Routledge.

Craig, W. J. and Elwood, S. (1998). How and Why Community Groups Use Maps and Geographic Information. Cartography and Geographic Information Systems, 25(2), 95-104

Craig, W., Harris, T. and Wiener, D. (2002). Community Participation and Geographic Information Systems. Boca Raton, FL: CRC Press.

Crampton, J. (2010). Cartographic Calculations of Territory. Progress in Human Geography, 35(1) 92-103.

Crampton, J. W. (2009). Cartography: Performative, Participatory, Political. Progress in Human Geography, 33(6), 840-848.

Crampton, J. (2003). The Political Mapping of Cyberspace. Chicago: University of Chicago Press.

Crampton, J. (2001). Maps as Social Constructions: Power, Communication and Visualization. Progress in Human Geography, 25(2), 235-252.

Crampton, J. and Krieger, J. (2006). An Introduction to Critical Cartography. ACME: An International E-Journal for Critical Geographies, 4(1), 11-33. Crocker, D.A. (2008). Ethics of Global Development: Agency, Capability, and Deliberative Democracy. New York: Cambridge University Press.

Crocker, D. (2006). Sen and Deliberative Democracy. In Alexander Kaufman (ed.), Capabilities Equality: Basic Issues and Problems. New York: Rouledge.

Crocker, D.A. (2003). Reckoning with Past Wrongs: A Normative Framework. In Carol A.L. Prager and Trudy Govier, Dilemmas of Reconciliation: Cases and Concepts. Waterloo, ON: Wilfrid Laurier University Press. 39-63. 
Crocker, D. (1991). Toward Development Ethics. World Development, 19(5), 457483.

Cunliffe, A. (2003). Reflexive Inquiry in Organizational Research: Questions and Possibilities. Human Relations, 56(8), 983-1003.

Damasio, A. (1994). Descartes' Error: Emotion, Reason, and the Human Brain. New York: G.P. Putnam and Sons.

Davies, P. (2012). 'Me', 'Me', 'Me': The Use of the First Person in Academic Writing and Some Reflections on Subjective Analyses of Personal Experiences. Sociology, 46(4), 744-752.

Debassige, L. and Pyne, S. (2012). Devilotion/Devolution: Profits from the Wilderness. In A. Corbiere, D. McGregor and C. Migwans (Eds.) Anishinaabewin NIIZH: Culture Movements, Critical Moments, 2011. M'Chigeeng, Ontario: Ojibwe Cultural Foundation.

Del Casino, V.J., and Hanna, S.P. (2006). “Beyond the 'Binaries': A Methodological Intervention for Interrogating Maps as Representational Practices." ACME: An International E-Journal for Critical Geographies, 4(1), 34-56.

Dennis, J.S. (1853). Report, Diary and Field Notes, Survey of the Indian Reserves on Lake Huron. Ministry of Natural Resources (Toronto), Ontario Crown Survey Records, Field Note Book (FNB) 832, Vol. 1.

Dennis, J.S. (1851). Report, Diary and Field Notes, Survey of the Indian Reserves on Lake Huron. Ministry of Natural Resources (Toronto), Ontario Crown Survey Records, Field Note Book (FNB) 832, Vol. 1.

Denzin, N. (2012). Triangulation 2.0. Journal of Mixed Methods Research, 6(2), 80-88.

Denzin, N.K. (2001). The Seventh Moment: Qualitative Inquiry and the Practices of a More Radical Consumer Research. The Journal of Consumer Research, 28(2), 324-330.

Denzin, N.K., Lincoln, Y.S. and Tuhiwai Smith, L. (2008). Handbook of Critical and Indigenous Methodologies. Thousand Oaks California: Sage.

Denzin N.K. and Lincoln, Y.S. (2005). The SAGE Handbook of Qualitative Research. Thousand Oaks: Sage Publications, 3rd ed.

Derrida, J. (2002). Force of Law: The 'Mystical Foundation of Authority'. In G. Anidjar (Ed.), Acts of Religion, New York: Routledge, 228-298.

Derrida, J. (1976). Of Grammatology. Baltimore: Johns Hopkins University Press.

De Saussure, F. (1959). Course in General Linguistics (trans. W. Baskin). New York: McGraw-Hill. 
Dewsbury, J.D. (2003). Witnessing Space: 'Knowledge without Contemplation. Environment and Planning A, 35, 1907-1932.

Dowd, G.E. (2002). War under Heaven: Pontiac, the Indian Nations $\mathcal{E}$ the British Empire. Baltimore: Johns Hopkins University Press.

Dower, N. (2000). Human Development - Friend or Foe to Environmental Ethics? Environmental Values, 9, 39-54.

Dunn, C. (2007). Participatory GIS: A People's GIS? Progress in Human Geography, 31, 617-638.

Durdu, P. O., N. Yalabik, and K. Cagiltay. (2009). A Distributed Online Curriculum and Courseware Development Model. Educational Technology and Society, 12(1), 230-248.

Dwyer, S. (1999). Reconciliation for Realists. Ethics and International Affairs, 13, 8198.

Edney, M.H. (2005). The Origins and Development of J. B. Harley's Cartographic Theories. Cartographica, Monograph, nos. 1 \& 2. Toronto: University of Toronto Press.

Egan, B. (2012). Sharing the Colonial Burden: Treaty-making and Reconciliation in Hul'qumi'num Territory. The Canadian Geographer, 56(4), 398-418.

Ellingson, L. (2009). Engaging Crystallization in Qualitative Research. Thousand Oaks, CA: SAGE.

Elwood, S. (2008). Volunteered Geographic Information: Future Research Directions Motivated by Critical, Participatory, and Feminist GIS. Geo Journal, 72,173-183.

Elwood, S. (2002a). GIS and Collaborative Urban Governance: Understanding their Implications for Community Action and Power. Urban Geography, 22(8), 73759 .

Elwood, S. (2002b). GIS use in Community Planning: A Multidimensional Analysis of Empowerment. Environment and Planning A, 34, 905-22.

Elwood, S., and Ghose, R. (2004). PPGIS in Community Development Planning: Framing the Organizational Context. Cartographica, 38(3-4), 19-33.

Elwood, S. and Leitner, H. (2003). GIS and Spatial Knowledge Production for Neighborhood Revitalization: Negotiating State Priorities and Neighborhood Visions. Journal of Urban Affairs, 25(2), 139-57. 
Elwood, S. and Leitner, H. (1998). GIS and Community-based Planning:

Exploring the Diversity of Neighborhood Perspectives and Needs. Cartography and Geographic Information Systems, 20(2), 77-88.

Ettlinger, N. (2004). Toward a Critical Theory of Untidy Geographies: The Spatiality of Emotions in Consumption and Production. Feminist Economics, 10(3), 21-54.

Evans, Dylan, (2002). The Search Hypothesis of Emotions. British Journal for the Philosophy of Science. 53, 497-509.

Farr, J. (2008). Locke, Natural Law, and New World Slavery. Political Theory, $36(4), 495-522$.

Finlay, L. and Gough. B. (Eds.) (2003). Reflexivity: A Practical Guide for Researchers in Health and Social Science, Oxford, Blackwell Publishing.

Fox, J., Suryanata, K. and Hershock, P. (2005). Mapping Communities: Ethics, Values, Practice. Honolulu, Hawaii: East-West Center

Friere, P. (2000). Pedagogy of the Oppressed. New York: The Continuum International Publishing Group.

Friere, P. and Faundez, A. (1989). Learning to Question. A Pedagogy of Liberation. New York: Continuum.

Game, A. and Metcalfe, A. (1996). Passionate Sociology. London: Sage.

Garfinkel, H. (1967). Studies in Ethnomethodology. Englewood Cliffs, NJ: PrenticeHall.

Gasper, D. (2002). Is Sen's Capability Approach an Adequate Basis for a Theory of Human Development? Review of Political Economy, 14(4), 435-61.

Genette, G. (1997). Paratexts: Thresholds of Interpretation. Cambridge: Cambridge University Press.

Gergen, K.J. \& Gergen, M.M. (1991). Toward Reflexive Methodologies. In F. Steier, (Ed.), Research and Reflexivity. Beverly Hills, CA: Sage, 76-95.

Ghose, R. (2001). Use of Information Technology for Community Empowerment: Transforming Geographic Information Systems into Community Information Systems. Transactions in GIS, 5(2), 141-63.

Ghose, R. and Elwood, S. (2003). Public Participation GIS and Local Political Context: Propositions and Research Directions. Urban and Regional Information Systems Association (URISA) Journal, 15 APA II:17-24. 
Giri, A. (2000). Rethinking Human Well-being: A Dialogue with Amartya Sen. Journal of International Development, 12, 1003-1018.

Goodchild, M. (2007a). Citizens as Voluntary Sensors: Spatial Data Infrastructure in the World of Web 2.0. International Journal of Spatial Data Infrastructures Research, 2, 24-32.

Goodchild, M. (2007b). Citizens as Sensors: The World of Volunteered Geography. GeoJournal, 69, 211-221.

Gore, C. (1997). Irreducible Social Goods and the Informational Basis of Amartya Sen's Capability Approach. Journal of International Development, 9(2), 235-250.

Gouldner, A.W. (1970). The Coming Crisis in Western Sociology, New York: Basic Books.

Gross, L. (2002). Bimaadiziwin, or the "Good Life," as a Unifying Concept of Anishinaabe Religion. American Indian Culture and Research Journal, 26(1), 15-32.

Hagerstrand, T. (1982). Diagram, Path and Project. Tijdschift voor Economische en Sociale Geografie, 73, 323-339.

Hagerstrand, T. (1976). Geography and the Study of Interaction between Nature and Society. Geoforum, 7, $329-334$.

Hagerstrand, T. (1975). Space, Time and Human Conditions, in A. Karlqvist, L. Lundquist, and F. Snickars (Eds.), Dynamic Allocation of Urban Space.

Farnborough, Hants: Saxon House.

Haq, ul M. (1995). Reflections on Human Development. New York: Oxford University Press.

Harley, J.B. (2001). The New Nature of Maps: Essays in the History of Cartography. Baltimore, Md.: Johns Hopkins University Press.

Harley, J. B. (1992). Rereading the Maps of the Columbian Encounter. Annals of the Association of American Geographers, 82(3), 522-542.

Harley, B. (1990). Cartography, Ethics and Social Theory. Cartographica, 27, 1-23.

Harley, B. (1989). Deconstructing the Map. Cartographica, 26, 1-20.

Harley, B. (1988). Secrecy and Silences: The Hidden Agenda of State Cartography in Early Modern Europe. Imago Mundi, 40, 57-76.

Harley, B. and Woodward, D. (1987). History of Cartography, Volume I. Chicago: University of Chicago Press. 
Harmon, K. (2003). You Are Here: Personal Geographies and Other Maps of the Imagination. New York, N.Y.: Princeton Architectural Press. [part 2, chp 4]

Harper, S. (2011). Statement of Apology - to Former Students of Indian Residential Schools on Behalf of the Government of Canada. Ottawa, 11 June. Available at http:/ / www.aadnc-aandc.gc.ca/eng/1100100015644

Harris, C. (2004). How Did Colonialism Dispossess? Comments from the Edge of Empire. Annals of the Association of American Geographers, 94, 165-182.

Harris, L. and Hazen, H. (2006). Power of Maps: (Counter) Mapping for Conservation. ACME: An International E-Journal for Critical Geographies, 4(1), 99130.

Harris, T. and Weiner, D. (2002). Implementing a Community-integrated GIS: Perspectives from South African Fieldwork. In W. Craig, T. Harris, and D. Weiner (Eds.), Community Participation and Geographic Information Systems. London: Taylor \& Francis, 246-58.

Harris, T. and Weiner, D. (1998). Empowerment, Marginalization and "Community-integrated" GIS. Cartography and Geographic Information Systems, 25(2): 67-76.

Harvey, F. (2000). The Social Construction of Geographical Information Systems. International Journal of Geographical Information Science, 14(8), 711-13.

Harvey, D. (1973). Social Justice and the City. Baltimore: Johns Hopkins University Press.

Harvey, F. and Chrisman, N. (2004). The Implication of Geography and Technology: The Social Construction of Geographic Information Systems. In S. D. Brunn, S. L. Cutter and J. W. Harrington, (Eds.), Geography and Technology, Dordrecht: Kluwer, 65-80.

Hatch, M.J. (1997). Irony and the Social Construction of Contradiction in the Humor of a Management Team. Organization Science, 8(3), 275-88.

Hayner, P. (2000). Unspeakable Truths: Confronting State Terror and Atrocities. Routledge: New York.

Heidegger, M. (1966). Discourse on Thinking: A Translation of Gelassenheit, by J.M. Anderson and E. Hans Freund. New York: Harper and Row.

Helgerson, R. (1993). Nation or Estate?: Ideological Conflict in the Early Modern Mapping of England. Cartographica, 301, 68-74.

Herb, G.H., Hakli, J., Corson, M.W., Mellow, N., Cobarrubias, S. and CasasCortes, M. (2009). Intervention: Mapping Is Critical! Political Geography, 28 (6). 
Herlihy, P.H. and G. Knapp. (2003). Maps of, by, and for the Peoples of Latin America. Human Organization 62, 303-14.

Hess, M. (2004). Spatial Relationships? Towards a Reconceptualization of Embeddedness. Progress in Human Geography, 28(2), 165-186.

Hirt, I. (2012). Mapping Dreams / Dreaming Maps: Bridging Indigenous and Western Geographical Knowledge, Cartographica, Special issue on Indigenous Cartography and Counter Mapping 47(2), 105-120.

Hoggett and Thompson (2002). Toward a Democracy of the Emotions. Constellations, Vol. 9, No. 1, 106-126.

Holland, R. (1999). Reflexivity. Human Relations, 52(4), 463-84.

Holmes, M. (2010). The Emotionalization of Reflexivity. Sociology, 44(1), 139-54.

Hughes, G. D., and D. C. Chafin. (1996). Turning New Product Development into a Continuous Learning Process. Journal of Product Innovation Management, 13(2), 89-104.

Irving, S. (2006). 'In a pure soil': Colonial anxieties in the work of Francis Bacon. History of European Ideas, 32: 249-262.

Irwin, R., Bickel, B., Triggs, V., Springgay, S., Beer, R., Grauer, K., Xiong, G. and Sameshima, P. (2009). The City of Richgate: A/r/tographic Cartography as Public Pedagogy, Jade, 28.1, 61-70.

Jankowski, P. and Nyerges, T. (2003). Toward a Framework for Research on Geographic Information-supported Participatory Decision-making. Urban and Regional Information Systems Association (URISA) Journal, 15 APA I: 9-18.

Jankowski, P. and Nyerges, T. (2001). GIS-supported Collaborative Decisionmaking: Results of an Experiment. Annals of the Association of American Geographers, 91(1), 48-70.

Jarvis, K. A. and Spearman, A.M. (1995). Geomatics and Political Empowerment: The Yaqui. Cultural Survival Quarterly, 18(4), 58-61.

Johnson, J., Cant, G., Howitt, R., Peters, E. (2007). Guest Editorial Creating Anticolonial Geographies: Embracing Indigenous Peoples' Knowledges and Rights. Geographical Research, 45(2), 117-120.

Johnson, J. and Murton, B. (2007). Re/placing Native Science: Indigenous Voices in Contemporary Constructions of Nature. Geographical Research, 45(2), 121-129.

Johnson, J., Louis, R. and Pramono, H. (2006). Facing the Future: Encouraging Critical Cartographic Literacies in Indigenous Communities. ACME: An International E-Journal for Critical Geographies, 4(1), 80-98. 
Jolly, M. (2007). Imagining Oceania: Indigenous and Foreign Representations of a Sea of Islands. The Contemporary Pacific, 19(2), 508-545.

Kamanamaikalani Beamer, B. and Kaeo Duarte, T. (2008). I Palapala No Ia Aina Documenting the Hawaiian Kingdom: A Colonial Venture? Journal of Historical Geography, 35, 66-86.

Kaul, S. (2001). The Partitions of Memory: The Afterlife of the Division of India. Bloomington, Indiana: Indiana University Press.

Kelly, J. (1999). Maori Maps. Cartographica, 36(2), 1-30.

Kelty, C. M. (2008). Two Bits: The Cultural Significance of Free Software. Experimental Futures: Technological Lives, Scientific Arts, Anthropological Voices, Durham, NC; London: Duke University Press.

Kitchin, R. (2008). The Practices of Mapping. Cartographica, 43(3), 211-216.

Kitchin, R. and Dodge, M. (2007). Rethinking Maps. Progress in Human Geography, 31(3), 331-344.

Klinsky, S., Sieber, R. and Meredith, T. (2008). Connecting Local to Global: Geographic Information Systems and Ecological Footprints as Tools for Sustainability. The Professional Geographer, 62(1), 84-102.

Korzybski, A. (1941). Science and Sanity: An Introduction to Non-Aristotelian Systems and General Semantics. Lakeville, Conn., International Non-Aristotelian Library Pub. Co.

Krygier, J. (2002). A Praxis of Public Participation GIS and Visualization. In W. Craig, T. Harris and D. Weiner (Eds.), Community Participation and Geographic Information Systems. London: Taylor \& Francis, 330-45.

Kwan, M. (2007). Affecting Geospatial Technologies: Toward a Feminist Politics of Emotion. The Professional Geographer, 59(1), 22-34.

Kwan, M. (2002). Feminist Visualization: Re-envisioning GIS as a Method in Feminist Geographic Research. Annals of the Association of American Geographers, 92(4), 645-61.

Labelle, W. (2006). Dokis: Since Time Immemorial. Field, Ontario: WFL Communications.

Laidler, G. J., Elee, P., Ikummaq, T., Joamie, E., and Aporta, C. (2010). Mapping Sea-ice Knowledge, Use, and Change in Nunavut, Canada (Cape Dorset, Igloolik, Pangnirtung). In I. Krupnik, C. Aporta, S. Gearheard, G.J. Laidler, and L. KielsenHolm, (Eds.), SIKU: Knowing Our Ice, Documenting Inuit Sea-Ice Knowledge and Use. Dordrecht: Springer. 
Laituri, M. and Harvey, L. (1995). Bridging the Space between Indigenous Ecological Knowledge and New Zealand Conservation Management using GIS. In D. Saunders, J. Craig, and E. Mattiske (Eds.), Nature Conservation: The Role of Networks, 122-31. New South Wales, Australia: Surrey Beatty.

Latham, A. and Conradson, D. (2003). Guest Editorial. Environment and Planning A, 35, 1901-1906.

Latour, B. (1988). The Politics of Explanation: An Alternative. In S. Woolgar (Ed.), Knowledge and Reflexivity: New Frontiers in the Sociology of Knowledge. London: Sage, 155-76.

Lauriault and Taylor (2005). Cybercartography and the New Economy: Collaborative Research in Action. In D.R. Fraser Taylor, (Ed.). Cybercartography Theory and Practice, Volume 4 in Modern Cartography Series, Amsterdam: Elsevier, chapter 8, 181-210.

Lawrence, R. J., and Despreés, C. (2004). Introduction: Futures of Transdisciplinarity. Futures, 36, 397-405.

Leakey, R. (2002). The Future of Conservation and Wildlife. Guy Stanton Ford Memorial Lecture, University of Minnesota, Minneapolis, November 1.

Ley, D. and Samuels, M. (1978). Humanistic Geography: Prospects and Problems. London: Croom Helm.

Lewis, G. M. (1998). Cartographic Encounters: Perspectives on Native American Mapmaking and Map Use. In Kenneth Nebenzahl, Jr., (Ed.), Lectures in the History of Cartography. Chicago: University of Chicago Press.

Louis, R. P. (2007). Can You Hear Us Now? Voices from the Margin: Using Indigenous Methodologies in Geographic Research. Geographical Research, 45(2), 130-139.

Louis, R.P. (2004). Indigenous Hawaiian Cartographer: In Search Of Common Ground. Cartographic Perspectives, 48, 7-22.

Louis, R.P., Johnson, J. T. and Pramono, A.H. (2012). Introduction: Indigenous Cartographies and Counter-Mapping, Cartographica Special Issue, 44, 77-79.

Lynch, M. (2000). Against Reflexivity as an Academic Virtue and Source of Privileged Knowledge. Theory. Culture \& Society, 17(3), 26-54.

McCall, M. K. (2003). Seeking Good Governance in Participatory-GIS: A Review of Processes and Governance Dimensions in Applying GIS to Participatory Spatial Planning. Habitat International, 27, 54-73. 
McCall, M.K. and Minang, P. (2005). Assessing Participatory GIS for Communitybased Natural Resource Management: Claiming Community Forests in Cameroon. Geographical Journal, 171, 340-56.

McGregor, A. (1999). Wiiswaaskingaa (Land of the Birch Trees). Whitefish River First Nation, Birch Island, Ontario Blue Moon Publishing.

McDonald, M.G. (2008 ). The Nature of Epiphanic Experience. Journal of Humanistic Psychology, 48(1), 89-115.

McNab, D. (1998). Who is on Trial? Teme-augama Anishnabai Land Rights and George Ironside Junior: Re-considering Oral Tradition. The Canadian Journal of Native Studies, XVIII(1), 117-133.

McVeigh, R. and Rolston, B. (2009). Civilising the Irish. Race and Class 51(1), 2-28.

Mandelbrot, B. (1983). The Fractal Geometry of Nature. New York: W.H. Freeman.

Marlatt, M. (2004). The Calamity of the Initial Surveys under the Robinson Treaties. In H. C. Wolfart (Ed.), Papers of the Thirty-fifth Algonquian Conference. Winnipeg: University of Manitoba Press. 281-335.

Martínez, E. and Reyes, C. (2005). Cybercartography and Society. In D.R. Fraser Taylor, (Ed.). Cybercartography - Theory and Practice, Volume 4 in Modern Cartography Series, Amsterdam: Elsevier, chapter 5, 99-121.

Massey, D. (2004). Geographies of Responsibility. Geografiska Annaler, 86B, 5-18.

Mobjörk, M. (2010). Consulting Versus Participatory Transdisciplinarity: A Refined Classification of Transdisciplinary Research. Futures, 42, 866-873.

Monmonier, M. (2006). From Squaw Tit to Whorehouse Meadow: How Maps Name, Claim, and Inflame. Chicago: University of Chicago Press.

Monmonier, M. (2004). Rhumb Lines and Map Wars: A Social History of the Mercator Projection. Chicago: University of Chicago Press.

Monmonier, M. (1995). Drawing the Line: Tales of Maps and Cartocontroversy. New York: Holt.

Monmonier, M. (1991). How to Lie with Maps. Chicago, IL: University of Chicago Press

Neverdeen Pieterse, (2009). Critical Holism and the Tao of Development. In, J. Nederveen Pieterse (Ed.), Development Theory: Deconstructions / Reconstructions. London: SAGE.

Nicholls, R. (2009). Research and Indigenous Participation: Critical Reflexive Methods. International Journal of Social Research Methodology, 12 (2), 117-126. 
Nowotny, H. (2009). Frontier Research in the Social Sciences and Humanities: What Does it Mean, What Can it Mean? ISSC-ICPHS workshop, Bergen 11 May 2009, "Humanities and Social Sciences: Intersections and Joined Paradigms", 1-8. Nussbaum, M. (2000). Women and Human Development. New York: Cambridge University Press.

Nussbaum, M. (2001). Upheavals of Thought: The Intelligence of Emotions. Cambridge; New York: Cambridge University Press.

Oakley, A. (2007). Fracture: Adventures of a Broken Body. Bristol: Policy Press.

Obermeyer, N. J. (1998a). The Evolution of Public Participation GIS. Cartography and Geographic Information Systems, 25(2), 65-66.

Obermeyer, N. J. (1998b). HUD's Community Connection for Local Empowerment. Paper presented at the NCGIA Special Meeting: Empowerment, Marginalization and Public Participation GIS, Santa Barbara, CA. Available at http: / / www.ncgia.ucsb.edu/varenius / ppgis / papers /

Offen, K. H. (2007). Creating Mosquitia: Mapping Amerindian Spatial Practices in Eastern Central America, 1629-1779, Journal of Historical Geography, 33, 254-282.

O'Riordan, T. and Voisey, H. (1998). The Political Economy of the Sustainability Transition. In T. O'Riordan and H. Voisey (Eds.), The Transition to Sustainability: The Politics of Local Agenda 21 in Europe, 3-30, London: Earthscan.

Palmer, M. (2012). Theorizing Indigital Geographic Information Networks, Cartographica, Special Issue on Indigenous Cartography and Counter Mapping, 47(2), 80-91.

Palmer, M. (2009). Engaging with Indigital Geographic Information Networks. Futures, 41, 33-40.

Palmer, M., Elmore, D., Watson, M., Kloese, K., Palmer, K. (2009). Xoa:dau to Maunkaui: Integrating Indigenous Knowledge into an Undergraduate Earth Systems Science Course. Journal of Geoscience Education, 57(2), 137-144.

Paris, G. (1997). Everyday Epiphanies. In P. Clarkson (Ed.), On the Sublime: In Psychoanalysis, Archetypal Psychology and Psychotherapy. London: Whurr, 85-95.

Parker, B. (2006). Constructing Community Through Maps? Power and Praxis in Community Mapping, The Professional Geographer, 58(4), 470-484.

Parks, L. (2001). Cultural Geographies in Practice: Plotting the Personal: Global Positioning Satellites and Interactive Media. Cultural Geographies, 8, 209-222.

Pahl, G., and Grote, K.H. (1996). Interdisciplinary Design: Knowledge and Ability Needed. Interdisciplinary Science Reviews, 21(4), 292-303. 
Paulus, T.M., Woodside, M. and Ziegler, M. F. (2010). "I Tell You, It's a Journey, Isn't It?" Understanding Collaborative Meaning Making in Qualitative Research. Qualitative Inquiry, 16(10) 852-862.

Pearce, M.W. (2008). Framing the Days: Place and Narrative in Cartography. Cartography and Geographic Information Science, 35, 17-32.

Pearce, M. and Louis, R. (2007). Mapping Indigenous Depth of Place. American Indian Culture and Research Journal, 32(3), 107-126.

Peluso, N. L. (1995). Whose Woods are These? Counter-Mapping Forest Territories in Kalimantan, Indonesia. Antipode, 27(4), 383-406.

Perkins, C. (2003). Cartography: Mapping Theory. Progress in Human Geography. 27, 341-351.

Perkins and Dodge, (2009). How Do We Map Geographies of Cartography? Paper presented to the International Cartographic Association Conference, Santiago, Chile, 15-21st November.

Peterson, R. (1999). Central African Voices on the Human-environment Relationship. Cultural and Spiritual Values of Biodoversity, UNEP, London, England: Intermediate Technology Publications.

Pickles, J. (2004). A History of Spaces: Cartographic Reason, Mapping and the Geocoded World. London; New York: Routledge.

Pickles, J. (1995a). Ground Truth: the Social Implications of Geographic Information Systems. New York: Guilford Press.

Pickles, J. (1995b). Representations in an Electronic Age: Geography, GIS, and Democracy. In J. Pickles (Ed.), Ground Truth: The Social Implications of Geographic Information Systems. New York: Guilford Press, 1-30.

Pollner, M. (1991). Left of Ethnomethodology: The Rise and Decline of Radical Reflexivity. American Sociological Review, 56, 370-80.

Poole, P. (1995). Indigenous Peoples, Mapping and Biodiversity Conservation: An Analysis of Current Activities and Opportunities for Applying Geomatics Technologies. Biodiversity Support Program Discussion Paper Series. Washington, DC: WWF, The Nature Conservancy, World Resources Institute.

Pressman, S. and Summerfield, G. (2002). Sen and Capabilities. Review of Political Economy, 14(4), 429-434.

Pulsifer, P., Laidler, G., Taylor, D.R.F. and Hayes, A. (2011). Towards an Indigenist Data Management Program: Reflections on Experiences Developing an Atlas of Sea Ice Knowledge and Use. The Canadian Geographer, 55(1), 108-124. 
Pulsifer, P. L., Hayes, A., Fiset, J-P., Taylor, D. R. Fraser (2008). An Open Source Development Framework in Support of Cartographic integration. In Peterson, M. (Ed.). International Perspectives on Maps and the Internet, Berlin: Heidelberg: Springer, 165-183.

Pulsifer, P. L., and D. R. F. Taylor. (2005). The Cartographer as Mediator: Cartographic Representation from Shared Geographic Information. In D. R. F. Taylor (Ed.), Cybercartography: Theory and Practice. Amsterdam: Elsevier, 149-179.

Pyne, S. (2013). The Role of Experience in the Iterative Development of the Lake Huron Treaty Atlas. In Taylor, D. R. F. (Ed.), Developments in the Theory and Practice of Cybercartography: Applications and Indigenous Mapping, Amsterdam: Elsevier (in press).

Pyne, S. (2013). Spatializing History: The Cybercartographic Atlas of the Lake Huron Treaty Process, in Karl Hele (Ed.), This is Indian Land: The Robinson Huron Treaties of 1850, (in press).

Pyne, S. (2009). A Cybercartographic Case Study of Lake Huron Treaty Relationships: The Treaties Module as a Reconciliation Tool, unpublished Ph.D. Thesis Proposal, Carleton University.

Pyne, S. (2008). Outline for a Postfoundational Rendering of the Lake Huron Treaty Relationship Process, unpublished Ph.D. comprehensive paper, Carleton University.

Pyne, S. (2007). The Political Economy of Relational Space, unpublished coursework paper, Carleton University.

Pyne, S. (2006). Full Responsible Reason and Good Development. Master's Thesis, unpublished, Carleton University.

Pyne, S. and Taylor, D.R.F. (2012). Mapping Indigenous Perspectives in the Making of the Cybercartographic Atlas of the Lake Huron Treaty Relationship Process, Cartographica, Special Issue on Indigenous Cartography and Counter Mapping, 47(2), 92-104.

Ramadier, T. (2004). Transdisciplinarity and its Challenges: The Case of Urban Studies. Futures 36, 423-439.

Rawls, J. (1957). Justice as Fairness. Journal of Philosophy, 54(22), 653-662.

Rawls, J. (1963). The Sense of Justice. Philosophical Review, 72(3), 281-305.

Rawls, J. (1968) Distributive Justice: Some Addenda. Natural Law Forum, 13, 51-71.

Rawls, J. (1971). A Theory of Justice. Cambridge, Massachusetts: Belknap Press. 
Richardson, L. (2000). Writing: A Method of Inquiry. In N. K. Denzin \& Y. S. Lincoln (Eds.), Handbook of Qualitative Research, 2nd ed. Thousand Oaks, CA: SAGE, 923-948.

Roberts, B. (2002). Biographical Research. Buckingham: Open University Press.

Robeyns, I. (2003). The Capability Approach: An Interdisciplinary Introduction Training Course preceding the 3rd International Conference on the Capability Approach, Pavia, Italy, on 6 September.

Robinson, J. (2004). Squaring the Circle? Some Thoughts on the Idea of Sustainable Development. Ecological Economics, 48(4), 369-84.

Rostow, W. (1971). The Stages of Economic Growth: A Non-Communist Manifesto. Cambridge, England: University Press.

Roth, R. (2009). The Challenges of Mapping Complex Indigenous Spatiality: From Abstract Space to Dwelling Space. Cultural Geographies, 16 (2), 207-227.

Ruddick, S. (1989). Maternal Thinking: Toward a Politics of Peace. Boston: Beacon Press.

Ruddick, S. (2009). Maternal Thinking: Philosophy, Politics, Practice. Toronto: Demeter Press

Rundstrom, R. A. (1995). GIS, Indigenous Peoples, and Epistemological Diversity. Cartography and Geographic Information Systems, 22(1), 45-57.

Rundstrom, R. (1993). The Role of Ethics, Mapping and the Meaning of Place in Relations Between Indians and Whites in the United States. Cartographica, 30(1), 21-29.

Rundstrom, R. (1991). Mapping, Postmodernism, Indigenous People and the Changing Direction of North American Cartography. Cartographica, 28(1), 1-12.

Sanford, C. and Rose, J. (2007). Characterizing eParticipation. International Journal of Information Management, 27(6), 406-421.

Saul, J. R. (2008). A Fair Country: Telling Truths about Canada. Toronto: Viking Canada.

Schlossberg, M. A., and Shuford, E. (2005). Delineating "Public" and "Participation" in PPGIS. Urban and Regional Information Systems Association (URISA) Journal, 16(2), 15-26.

Schuurman, N. (2001). Critical GIS: Theorizing an Emerging Discipline. Cartographica, 36(4), 1-108. 
Schuurman, N. (2000). Trouble in the Heartland: GIS and its Critics in the 1990s. Progress in Human Geography, 24(4), 569-90.

Schuurman, N. and Kwan, M. (2004). Introduction: Taking a Walk on the Social Side of GIS. Cartographica, 39(1), 1-4.

Seeger, C.J. (2008). The Role of Facilitated Volunteered Geographic Information in the Landscape Planning and Site Design Process, Geojournal, 72, 199-213.

Semali, L.M. and Kincheloe, J.L. (1999). What is Indigenous Knowledge: Voices from the Academy. New York: Falmer Press.

Sen, A. (1999). Development as Freedom. Oxford: Oxford University Press.

Sen, A. (1992). Inequality Re-examined. Cambridge, Mass.: Harvard University Press.

Shaule, S. (2002). “The Disputed Boundaries of the 1923 (Williams) Treaties. Unpublished MA thesis, Trent University.

Shaule, S. (2013). The Constellations Reveal Themselves One Star at a Time: The Mississauga \& Chippewa Land Claim, 1890-1900. In K. Hele (ed). This is Indian Land: The Robinson Huron Treaties of 1850, (in press).

Sheppard, E. (1995). GIS and Society: Towards a Research Agenda. Cartography and Geographic Information Systems, 22(1), 5-16.

Shotter, J. Conversational Realities: Constructing Life through Language. Thousand Oaks, CA: Sage, 1993.

Sieber, R. E. (2007). Spatial Data Access by the Grassroots. Cartography and Geographic Information Science, 34(1), 47-62.

Sieber, R. (2006). Public Participation Geographic Information Systems: A Literature Review and Framework. Annals of the Association of American Geographers, 96(3), 491-507.

Sieber, R. (2004). A Public Participation GIScience? Cartographica, 38 (3\&4), 1-7.

Sills, D. (1968). International Encyclopedia of the Social Sciences. MacMillan Co. and Free Press.

Sletto, B. (2009). Indigenous People Don't have Boundaries': Reborderings, Fire Management, and Productions of Authenticities in Indigenous Landscapes, Cultural Geographies, 16 (2), 253-277.

Smith, A. D. (2004). House Arrest and Piano. New York, NY: Anchor. 
Smith, A. (1976). An Inquiry into the Nature and Causes of the Wealth of Nations. Oxford (Oxfordshire): Clarendon Press; New York: Oxford University Press.

Sparke, M. (2005). In the Space of Theory: Postfoundational Geographies of the Nationstate. Minneapolis: University of Minnesota Press.

Sparke, M. (1998). A Map that Roared and an Original Atlas: Canada, Cartography, and the Narration of Nation. Annals of the Association of American Geographers, 88(3), 463-495.

Steier, F. (Ed.) (1991). Research and Reflexivity. Beverly Hills, CA: Sage.

Swyngedouw, E. (1997). Neither Global nor Local. Glocalisation and the Politics of Scale. In K. Cox, Spaces of Globalization: Reasserting the Power of the Local, 137166. New York: Guilford Press.

Talen, E. (2000). Bottom-up GIS: A New tool for Individual and Group Expression in Participatory Planning. Journal of the American Planning Association, 66(3), 279-94.

Taylor, D. R. F. (forthcoming 2013). Fifty Years of Cartography. The Cartographic Journal, 50(2).

Taylor, D. R. F. (2009). Maps, Mapping and Society: Some New Directions. In Proceedings of Global Map Forum, Tskuba, Japan: Geographical Survey Institute, 32-35.

Taylor, D. R. F. (2005). Cybercartography: Theory and Practice, Volume 4 in Modern Cartography Series, Amsterdam: Elsevier, 574.

Taylor, D. R. F. (2003). The Concept of Cybercartography. In M. P. Peterson (ed.), Maps and the Internet, Amsterdam: Elsevier, 405-420.

Taylor D.R.F. (1997). Maps and Mapping in the Information Era, Keynote address to the 18th ICA Conference, Stockholm. In L. Ottoson (Ed.), Proceedings, Vol. 1, 110 .

Taylor, D. R. F. (1991). A Conceptual Basis for Cartography/New Directions for the Information Era. Cartographica, 28(4), 1-8.

Taylor, D. R. F. (1985). Education and Training in Contemporary Cartography. Wiley, Chichester.

Taylor, J. (2008). Naming the Land: San Countermapping in Namibia's West Caprivi. Geoforum, 39, 1766-1775.

Taylor, D.R.F. and Pyne, S. (2010). The History and Development of the Theory and Practice of Cybercartography. International Journal of Digital Earth, 3(1), 1-14. 
Taylor, D.R.F. and McKenzie, F. (1992). Development from Within: Survival in Rural Africa. London; New York: Routledge.

Taylor, D. R. F. and Caquard, S. (Guest Eds.) (2006). Special Issue on Cybercartography, Cartographica, 41(1), 1-5.

Telford, R. M. 1997. The Nefarious and Far-ranging Interests of Indian Agent and Surveyor John William Keating, 1837 to 1869. In D. H. Pentland (Ed.) Papers of the Twenty-eighth Algonquian Conference. Winnipeg: University of Manitoba Press, 372-402.

Thrift, N. (2004a). Intensities of Feeling: Towards a Spatial Politics of Affect. Geografiska Annaler, 86B, 57-78.

Thrift, N. (2004b). Performance and Performativity: A Geography of Unknown Lands. In Duncan, J., Johnson, N. and Schein, R. (Eds.) A Companion to Cultural Geography. Blackwell, Oxford, 121-136.

Thrift, N. (2000). Afterword. Environment and Planning D: Society and Space, 18, 213-255.

Thrift, N. (1996). Spatial Formations. London: Sage Publications.

Tobias, T. N. (2000). Chief Kerry's Moose: A Guidebook to Land Use and Occupancy Mapping, Research Design, and Data Collection. Vancouver: Union of BC Indian Chiefs: Ecotrust Canada.

Topfer, K. (1999). Opening address, in UNEP (1999). Cultural and Spiritual Values of Biodoversity. London, England: Intermediate Technology Publications.

Treuer, A. (2011). The Assassination of Hole in the Day. Saint Paul: Minnesota Historical Society Press.

Tuan, Y-F. (1977). Space and Place: The Perspective of Experience. London: Edward Arnold.

Tuhiwai-Smith, L. (1999). Decolonizing Methodologies; Research and Indigenous Peoples. London: Zed Books.

Turnbull, D. (2007). Maps, Narratives, and Trails: Performativity, Hodology, and Distributed Knowledges in Complex Adaptive Systems: An Approach to Emergent Mapping. Geographical Research, 45, 140-149.

Turnbull, D. (2000). Masons, Tricksters, and Cartographers: Comparative Studies in the Sociology of Scientific and Indigenous Knowledge. Amsterdam: Harwood Academic.

Turnbull, D. (1993). Maps are Territories: Science is an Atlas: A Portfolio of Exhibits. Chicago: University of Chicago Press. 
UNEP (1999). Cultural and Spiritual Values of Biodoversity. London, England: Intermediate Technology Publications.

van Staveren, I. (2001). The Values of Economics: An Aristotelian Perspective. London: Routledge.

Varvis, S. (1983). Humanism and the Scientific Revolution: Bacon's Rejection of Aristotle. Comitatus: A Journal of Medieval and Renaissance Studies, 14(1), 60-79.

Verganti, R. (2011). Radical Design and Technology Epiphanies: A New Focus for Research on Design Management. Journal of Product Innovation Management, 28, 384-388.

Wainwright, J. and Bryan, J. (2009). Cartography, Territory, Property: Postcolonial Reflections on Indigenous Counter-mapping in Nicaragua and Belize, Cultural Geographies, 16, 153-178.

Walker, B. L. (2007). Mamiya Rinzo and the Japanese Exploration of Sakhalin Island: Cartography and Empire. Journal of Historical Geography, 33, 312.

Watson, T. (1995). Rhetoric, Discourse and Argument in Organizational Sense Making: A Reflexive Tale. Organizational Studies, 16(5), 805-21.

Winichakul, T. (1997). Siam Mapped: A History of the Geo-Body of a Nation, Honolulu, 1997, 131.

Winters, N., and Y. Mor. (2008). IDR: A Participatory Methodology for Interdisciplinary Design in Technology Enhanced Learning. Computers $\mathcal{E}$ Education, 50, 579-600.

Withers, R. (2008). Descartes' Dreams. Journal of Analytical Psychology, 53, 691-709.

Wittgenstein, L. (1980). Culture and Value. Translated by Peter Winch. Chicago: The University of Chicago Press.

Wittgenstien, L. (1953). Philosophical Investigations. Translated by G.E.M. Anscombe. Oxford: Blackwell.

Wood, D. (1992). The Power of Maps. New York: Guilford.

Wood, D. and Fels, J. (2008). The Natures of Maps: Cartographic Constructions of the Natural World. Chicago: University of Chicago Press.

Wood, D. and Fels, J. (1986). Designs on Signs: Myth and Meaning in Maps. Cartographica, 23(3), 54-103.

Woodward, D. and Lewis, G.M. (2000). Cartography in the Traditional African, American, Arctic, Australian, and Pacific Societies. Baltimore: University of Chicago Press. 
Woodward, D. and Lewis, G. M. (1998). The History of Cartography: Cartography in the Traditional African, American, Arctic, Australian, and Pacific Societies. Vol. 2, Book 3. Chicago: The University of Chicago Press.

Wright, D. J., Goodchild, M.F. and Proctor, J.D. (1997). Demystifying the Persistent Ambiguity of GIS as "Tool" versus "Science". Annals of the Association of American Geographers, 87(2), 346-62.

Wu, C.J. and Isaksson, K. (2008). Participatory Mapping as a Tool for Capturing Local Perspectives on Cultural Landscape: A Case Study of Ostlänken. Report for School of Architecture and the Built Environment Urban Planning and Environment, Stockholm. Available at: www.includemistra.org.

Vidal, A., and T.G. Anderson. (1849). Report of Commissioners A. Vidal \& T.G. Anderson on visit to the Indians on the north shores of lakes Huron and Superior for purposes of investigating their claims to territory bordering on those Lakes. Library and Archives Canada (Ottawa). RG 1, Vol. 266, Reel C-12652, 4.

Young, I. (2000). Inclusion and Democracy. Oxford; New York: Oxford University Press.

Young, J. (2010). Final Co-op Student Report, Geomatics and Cartographic Research Centre, Carleton University, unpublished report.

Zagzebski, L. (1996). Virtues of the Mind: An Inquiry into the Nature of Virtue and the Ethical Foundations of Knowledge. New York: Cambridge University Press. 
Appendices 


\section{Appendix A}

\section{Full Text of the Seven Fires Prophecy}

Seven prophets came to the Anishinabe. They came at a time when the people were living a full and peaceful life on the North Eastern coast of North America. These prophets left the people with seven predictions of what the future would bring. Each of the prophecies was called a fire and each fire referred to a particular era of time that would come in the future. Thus, the teachings of the seven prophets are now called the "Seven Fires."

The first prophet said to the people, "In the time of the First Fire, the Anishinabe nation will rise up and follow the sacred shell of the Midewiwin Lodge. The Midewiwin Lodge will serve as a rallying point for the people and its traditional ways will be the source of much strength. The Sacred Megis will lead the way to the chosen ground of the Anishinabe. You are to look for a turtle shaped island that is linked to the purification of the earth. You will find such an island at the beginning and at the end of your journey. There will be seven stopping places along the way. You will know the chosen ground has been reached when you come to a land where food grows on water. If you do not move you will be destroyed."

The second prophet told the people, "You will know the Second Fire because at this time the nation will be camped by a large body of water. In this time the direction of the Sacred Shell will be lost. The Midewiwin will diminish in strength, a boy will be born to point the way back to the traditional ways. He will show the direction to the stepping stones to the future of the Anishinabe people.

The third prophet said to the people. "In the Third Fire, the Anishinabe will find the path to their chosen ground, a land in the west to which they must move their families. This will be the land where food grows on water.

The Fourth Fire was originally given to the people by two prophets. They come as one. They told of the coming of the Light Skinned race.

One of the prophets said, "You will know the future of our people by the face the Light Skinned race wears. If they come wearing the face of brotherhood then there will come a time of wonderful change for generations to come. They will bring new knowledge and articles that can be joined with the knowledge of this country, in this way, two nations will join to make a mighty nation.

This new nation will be joined by two more so that four will form the mightiest nation of all. You will know the face of the brotherhood if the light skinned race comes carrying no weapons. If they come bearing only their knowledge and a hand shake."

The other prophet said," Beware if the Light Skinned race comes wearing the face of death. You must be careful because the face of brotherhood and the face of 
death look very much alike. If they come carrying a weapon...beware. If they come in suffering... They could fool you. Their hearts may be filled with greed for the riches of this land. If they are indeed your brothers, let them prove it. Do not accept them in total trust. You shall know that the face they wear is one of death if the rivers run with poison and the fish become unfit to eat. You shall know them by these many things.

The Fifth Prophet said, "In the time of the Fifth Fire there will come a time of great struggle that will grip the lives of all Native people. At the warning of this Fire there will come among the people one who holds a promise of great joy and salvation. If the people accept this promise of a new way and abandon the old teachings, then the struggle of the Fifth Fire will be with the people for many generations. The promise that comes will prove to be a false promise. All those who accept this promise will cause the near destruction of the people."

The prophet of the Sixth Fire said, "In the time of the Sixth Fire it will be evident that the promise of the Fifth Fire came in a false way. Those deceived by this promise will take their children away from the teachings of the Elders, grandsons and grand-daughters will turn against the Elders. In this way, the Elders will lose their reason for living... they will lose their purpose in life. At this time a new sickness will come among the people. The balance of many people will be disturbed. The cup of life will almost be spilled. The cup of life will almost become the cup of grief."

At the time of these predictions, many people scoffed at the prophets. They then had medicines to keep away sickness. They were then healthy and happy as a people. These were the people who chose to stay behind in the great migration of the Anishinabe. These people were the first to have contact with the Light Skinned race. They would suffer the most.

When the Fifth Fire came to pass, a great struggle did indeed grip the lives of all Native people. The Light Skinned race launched a military attack on the Indian people throughout the country aimed at taking away their land and their independence as a free and sovereign people. It is now felt that the false promise that came at the end of the Fifth Fire was the materials and riches embodied in the way of life of the light skinned race. Those who abandoned the ancient ways and accepted this new promise were a big factor in causing the near destruction of the Native people of this land.

When the Sixth Fire came to be, the words of the prophet rang true as the children were taken away from the teachings of the Elders. The boarding school era of "civilizing" Indian Children had begun. The Indian language and religion were taken from the children. The people started dying at an early age... they had lost their will to live and their purpose in living.

In the confusing times of the Sixth Fire, it is said that a group of visionaries came among the Anishinabe. They gathered all the priests of the Midewiwin Lodge. They told the priests that the Midewiwin Way was in danger of being destroyed. They gathered all the sacred bundles. They gathered all the scrolls that recorded 
the ceremonies. All these things were placed in a hollowed out log from the ironwood tree. Men were lowered over a cliff by long ropes. They dug a hole in the cliff and buried the log where no one could find it. Thus the teachings of the Elders were hidden out of sight but not out of memory. It was said that when the time came that the Indian people could practice their religion without fear that a little boy would dream where the Ironwood log, full of the Sacred Bundles and Scrolls were buried. He would lead his people to the place.

The Seventh Prophet that came to the people long ago was said to be different from the other prophets. He was young and had a strange light in his eyes. He said, " In the time of the Seventh Fire, New People will emerge. They will retrace their steps to find what was left by the trail. Their steps will take them to the Elders who they will ask to guide them on their journey. But many of the Elders will have fallen asleep. They will awaken to this new time with nothing to offer. Some of the Elders will be silent out of fear. Some of the Elders will be silent because no one will ask anything of them. The New People will have to be careful in how they approach the Elders. The task of the New People will not be easy.

If the New People will remain strong in their Quest, the Water Drum of the Midewiwin Lodge will again sound its voice. There will be a Rebirth of the Anishinabe Nation and a rekindling of old flames. The Sacred Fire will again be lit.

It is at this time that the Light Skinned race will be given a choice between two roads. If they choose the right road, then the Seventh Fire will light the Eighth and final Fire, an eternal Fire of peace, love, brotherhood and sisterhood. If the light skinned race makes the wrong choice of roads, the destruction which they brought with them in coming to this country will come back at them and cause much suffering and death to all the Earth's people.

Author: Edward Benton Banai, a Midewiwin teacher and Grand Chief of the Three Fires Midewiwin Lodge, from Lac Courte Oreilles, Wisconsin.

Available at: http:// www.manataka.org/page20.html. 


\section{Appendix B}

\section{List of Presentations}

Pyne, S. (2008). The Treaties Module of the 'Living' Cybercartographic Atlas of Indigenous Perspectives and Knowledge: Bringing Memories to Life. Presented by Stephanie Pyne at the 3rd Annual Conference of the Communication Graduate Caucus, School of Journalism and Communication, Memory as Medium: Experience, Exchange, Representation, Carleton University, Ottawa, March 13-14 (See Presentation 1, Appendix D).

Pyne, S. (2008). Linking Cybercartography and Indigenous Tourism: The Reconciliation Trail. Presented by Stephanie Pyne at the Canadian Association of Geographers Annual Meeting, Quebec City, May, (received Student Study Group Award; see Presentation 2, Appendix D).

Pyne, S., (2008). A Guided Tour of the Treaties Module: Building Awareness and Bridging Relationships. Presented by Stephanie Pyne at the GeoTec Conference: People, Policies and Progress. Bringing together Academia, Government and Industry, Ottawa, June (see Presentation 3, Appendix D).

Pyne, S. (2009). Designing Cybercartographic Atlases as Reconciliation Tools. Presented by Stephanie Pyne at the Circle of All Nations Workshop Sustainable Relationships: Reconciliation and Integration, Victoria Island, Ontario, May 2224.

Pyne, S. (2009). A Cybercartographic Case Study of the Lake Huron Treaty Relationship Process: Building Awareness to Bridge Relationships. Presented by Stephanie Pyne at the Canadian Association of Geographers Annual Meeting, Carleton University, Ottawa, May 27.

Pyne, S. (2009). The Cybercartographic Atlas of the Lake Huron Treaty. Presented by Stephanie Pyne at Manitoulin High School, Manitoulin Island, Ontario, October 19.

Pyne, S. (2009). The Cybercartographic Atlas of the Lake Huron Treaty. Presented by Stephanie Pyne at M'Chigeeng Adult High School, M'Chigeeng First Nation, Manitoulin Island, Ontario, October 19.

Pyne, S. (2009). The Little Land We Yet Possess: Surveying the Robinson Huron Reserves. Ojibwe Cultural Foundation Special Presentation by Stephanie Pyne along the theme of the month's Exhibit "Creation, Land, Treaty: From Sacred to Profane", M'Chigeeng First Nation, Manitoulin Island, Ontario, October 19, 2009.

Pyne, S. (2009). The Making of the Cybercartographic Atlas of the Lake Huron Treaty. Presented by Stephanie Pyne at the National Collaborating Centre for Aboriginal Health Atlas Information Session, Vancouver, October 30. 
Pyne, S., Taylor, D.R.F., and Caquard, S. (2009). The Emerging Role of Art in Cybercartography: Conveying Indigenous and Critical Perspectives in the Treaties Module. Presented by D. R. F. Taylor at ICC 2009 24th International Cartography Conference, November 15 to 21, 2009, Santiago,

Chile.

Pyne, S. (2009). Designing and Developing the Cybercartographic Atlas of the Lake Huron Treaty. Presented by Stephanie Pyne at the MNR Ontario Aboriginal Working Group Meeting, North Bay, Ontario, December 11.

Pyne, S. (2010). Getting Colonialism at the Roots: The Cybercartographic Atlas of the Lake Huron Treaty. Presented by Stephanie Pyne at the American Association Of Geographers Annual Meeting, Washington, D.C., April 14 to 18.

Pyne, S. (2010). Creating New Spaces of History in the Cybercartographic Atlas of the Lake Huron Treaty. Presented by Stephanie Pyne at the Canadian Association of Geographers Annual Meeting, Regina, Saskatchewan, June 2-5.

Brauen, G. and Pyne, S. (2010). Reflections on Project Roles and Technologies: The Cybercartographic Atlas of the Lake Huron Treaty. Presented by Glenn Brauen and Stephanie Pyne at the Canadian Association of Geographers Annual Meeting, Regina, Saskatchewan, June 2-5, 2010.

Pyne, S. (2010). The Cybercartographic Atlas of the Lake Huron Treaty: Mapping the History. Presented by Stephanie Pyne at the Engaging Indigenous Communities: Resources, Rebellions, and Resurgence Conference, Sault Ste. Marie, Ontario, August 10-13 (see Appendix D for video recordings of conference event - Teddy Syrette reading 1849 petition).

Pyne, S. (2011). Incorporating Artists' Perspectives into the Atlas of the Lake Huron Treaty. Presented by Stephanie Pyne at The Great Lakes Research Alliance for the Study of Aboriginal Arts and Cultures (GRASAC) Research Conference 2011, June 9-11, Ojibwe Cultural Foundation (OCF) M'Chigeeng First Nation, Manitoulin Island.

Pyne, S. and Taylor, D.R.F. (2011). Reconciliatory Ethics in the Project to Create the Cybercartographic Atlas of the Lake Huron Treaty Relationship Process. Paper presented by D. R. F. Taylor at the Mapping Processes and Practices: Arts, Maps and Society Workshop, International Cartographic Association Commission on Maps and Society Working Group on Art and Cartography, Paris, France July 2.

Pyne, S. (2012). Unveiling the Working Atlas of the Lake Huron Treaty. Presented by Stephanie Pyne at the official Atlas Launch Gathering, Carleton University, Ottawa, May 28. 


\section{Appendix C}

\section{Phase II and Phase III \\ Selected Fieldwork Summary}

\section{Phase I}

Date:

July $5-7,2009$

Place:

Shebeshekong Bay in Shawanaga Traditional Territory

Fieldwork:

Together with Atlas volunteers, Jesse Young and Jenica Shivkumar, we visited with, regional historian and archaeologist, Bill Allen, and gathered photographic media for Survey Journeys interactive maps (see Appendix D for digital slideshow of photographic media).

Date:

July 8-9, 2009

Place:

Batchewana First Nation

Fieldwork:

Attended Chiefs of Ontario AGM and spoke with several chiefs from the treaty region and from elsewhere in Ontario (community leaders) to inform them about the commencement of phase II of the Lake Huron Treaty Atlas project and to invite participation.

Date:

July 10, 2009

Place:

Shingwauk University, Bawating (Sault Ste. Marie)

Fieldwork: $\quad$ Met with Darrell Boisseneau (Director) and the late Blaine Belleau (both from Gitigaan-ziibing or Garden River First Nation) to inform them of the commencement of the Lake Huron Treaty Atlas and to discuss potential directions for Atlas design and development.

Date:

July 10, 2009

Place:

Serpent River First Nation

Fieldwork:

Meeting with Chief Isadore Day (Wiindawtegowinini) in his capacity as Lake Huron Treaty Commissioner (community leader) to inform him of the commencement of the Lake 
Huron Treaty Atlas and to discuss directions for Atlas design and development.

Date: $\quad$ July 31 - August 2, 2009

Place: $\quad$ Kitigan Zibi, Quebec

Fieldwork: $\quad$ Informed people at the Circle of All Nations Annual Spiritual Gathering about the commencement of the Lake Huron Treaty Atlas and discussed directions for Atlas design and development.

Date:

Place:

Fieldwork:

Date:

Place:

Fieldwork:

Date:

Place:

Fieldwork:

Date:
August 3 to 5, 2009

Serpent River First Nation

Visited for a day during Community Days, informed community members and band council employees about the commencement of the Lake Huron Treaty Atlas and discussed directions for Atlas design and development. Also met with and Lake Huron Treaty Commissioner, Gimaah Isadore Day (Wiindawtegowinini), to provide updates on atlas development progress and discuss future directions.

August 6, 2009

North Bay, Ontario

Meeting with Norm Dokis to discuss the Atlas project and construction of a potential Gimaah Michael (Eagle) Dokis Interactive Biography Map.

August 28-30, 2009

Gitigaan-ziibing (Garden River First Nation)

Participated in Three Fires Society Midewiwin Lodge 2009 summer ceremonies. Met with the late Blaine Belleau and Wilmer Noganosh and discussed Atlas design and development. Informed community members about Atlas work and invited participation. Planned with Blaine Belleau to return in the winter and begin construction of the potential "Reading Between the Lines" Interactive Map. 
Place:

Fieldwork:

Date:

Place:

Fieldwork:

Date:

Place:

Fieldwork:

Date:

Place:

Fieldwork:

Date:
Dokis First Nation

Atlas content development. Together with Norm Dokis, gathered geo-referenced photographic media for Michael (Eagle) Dokis Interactive Biography map. Informed community members about atlas and invited participation.

February 26-27, 2010

Nsawakamok (Sudbury), Ontario

Gathered video footage and photos of presenters at Anishinaabewin: Modes of Knowing, Ways of Life (conference hosted by the Ojibwe Cultural Foundation) for potential inclusion in the Atlas, in addition to making new community-based atlas contributor contacts, disseminating information about the Atlas project and engaging in some Atlas design discussions.

May 24, 2010- May 30, 2010

Gitigaan-ziibing (Garden River) and Batchewana First Nations

Visited and worked with several community members gathering video footage and photos for potential inclusion in the Atlas, including the late Blaine Belleau; disseminated information about the Atlas project, and discussed potential contributions.

February 25 - 26, 2011

Sudbury, Ontario

Gathered video footage and photos of presenters Anishinaabewin: Culture Movements, Critical Moments (hosted by the Ojibwe Cultural Foundation) for potential inclusion in the Atlas, in addition to making new community-based atlas contributor contacts, disseminating information about the Atlas project and engaging in atlas design discussions.

March 11, 2011 
Place:

Fieldwork:

Date:

Place:

Fieldwork:

Date:

Place:

Fieldwork:

Date:

Place:

Fieldwork:

Date:

Place:
Union of Ontario Indians (UOI Head Office, Nipissing First Nation)

Video documentation of Mother Earth Water Walk Presentation by Elder Josephine Mandamin (hosted by Akiing-bemosejig Environmental Committee of the Union of Ontario Indians (UOI)), in addition to making new community-based atlas contributor contacts, disseminating information about the Atlas project and engaging in some atlas design discussions.

August 3-8, 2011

Bawating (Sault Ste. Marie)

Met with various individuals, including Shingwauk Residential Schools Centre collaborators, Phil Belfy and the late Blaine Belleau, to discuss Atlas design and development and to gather content (see Appendix D. for video clip of helicopter ride over Bawating).

August 9, 2011

Manitowaning

Recorded video footage of the Bondhead Treaty Commemorative Event for inclusion in various Atlas maps. Disseminated information about the Atlas project and engaged in some atlas design-related discussions.

October 26-29, 2011

Ottawa, Ontario

Video and photographic documentation of presentations at Native American Art Studies Association Biennial Conference and information dissemination to various delegates regarding Atlas project.

November 10, 2011

Ottawa, Ontario 
Fieldwork:

Video documentation of Unveiling the Three Watchmen sculpture by James Hart at the National Art Gallery for eventual inclusion in the Gathering Places Map.

Date:

Place:

Fieldwork:

Date:

Place:

Fieldwork:

Phase III

Date:

Place:

Fieldwork:
April 12, 2012

Ottawa, Ontario

Video documentation of "Landscapes of Desire: Anna's Sketches in Upper Canada as the Colonial Eye turns north by Crystal Migwans at the Carleton University Art History Student Conference" (see Appendix D for digital video recording of presentation).

July $7-23,2012$

Lake Huron Treaty region, including Manitoulin Island, Thessalon and Sagamok First Nation

In-the-field Atlas launch, including information dissemination; video documentation and informing people about Atlas project and purpose at the International Joint Commission public meeting in Wewebijiwang (Little Current) on July 15, 2012.

September 13, 2012

Adults in Motion (Garden River First Nation Education Unit), Gitigaan-ziibing (Garden River First Nation)

Joanne Thiessen (Co-op Co-ordinator), Bonnie Pregent (teacher) and I met for an initial outreach meeting to discuss a potential map project involving high school co-op students. At this meeting, I provided an introduction to the Lake Huron Treaty Atlas, highlighting its collaborative learning potential. The teachers remembered my presentation at "Engaging Indigenous Communities: Resources, Rebellions, and Resurgence" held in Bawating in August 2010, during phase II of the Atlas project under a SSHRC Standard Research Grant. The teachers and manager were quite enthusiastic and put forward some initial ideas regarding a collaborative mapping project. The teachers said that they would follow up on the meeting by selecting a student who they felt 
would work well on the project and who would be able to earn co-op credits for their participation.

Date:

Place:

Fieldwork:

Date:

Place:

Fieldwork:

Date:

Place:

Fieldwork:

Date:

Place:

Fieldwork:
September 25, 2012

Central Algoma High School, Desbarats, Ontario

At this meeting with Charlie Cook (guidance counselor), I provided an introduction to the Lake Huron Treaty Atlas, highlighting its collaborative learning potential. We discussed Atlas and the outreach project ideas and learned about some of the high school learning programs that could apply, including the Native Arts and co-op programs. Charlie put forward the idea of initiating some form of class mapping project.

October 6, 2012

Ojibwe Cultural Foundation, M'Chigeeng, Manitoulin Island, Ontario

Video recorded presentation by Elder Terry Debassige at "150 Years Later - Attempting to Understand the 1862 Manitoulin Treaty" and made outreach connections with students from Lakeview School, elders, and historians who attended the event. Met with Elder Lewis Debassige, atlas contributor, following symposium.

October 11, 2012

Ottawa, Ontario

Patricia Kennedy and I discussed ongoing Atlas design, development and content-related issues, including potential for Patricia to mentor students and other collaborators in the area of archival research and cartographic approaches to the creation of finding aids in the design of the Governance Paper Trail Map.

October 11, 2012

Ottawa, Ontario

Video recorded the following event for inclusion in the Atlas: Roundtable - The Four Wars of 1812: Dr. Sid Hart, Senior 
Historian at the Smithsonian's National Portrait Gallery (American perspective), Dr. Andrew Lambert, Laughton Professor of Naval History in the Department of War Studies at Kings College, London. (British perspective), Mr. Alan Corbiere, Program Coordinator for the Anishinaabemowin Revitalization Program at Lakeview Elementary School in M'Chigeeng (Native-American perspective), Dr. Peter MacLeod, pre-Confederation historian at the Canadian War Museum and curator of 1812 (Canadian perspective). Attended post event gathering and discussed Atlas project with attendees.

Date:

October 12, 2012

Place:

Carleton University, Ottawa, Ontario

Fieldwork:

Atlas Design and Development - Conversion of Atlas to Couch DB atlas software framework. Glenn Brauen and I discussed issues related to timing of conversion, tasks to be completed and implications for scope of Outreach activities.

Date: $\quad$ October 13, 2012

Place: $\quad$ Gatineau, Quebec

Fieldwork: $\quad$ Stephanie Pyne and Jacob Wawatie discussed issues at the intersection of Atlas design and development and Algonquin traditional knowledge, with a focus on the Pike Head Teaching.

Date: $\quad$ October 20, 2012

Place: $\quad$ Algoma University, Sault Ste. Marie, Ontario

Fieldwork: $\quad$ Video recorded presentations by Isabelle Meawasige, Alan Corbiere, Barbara Nolan and Guy Dumas at Elders Gathering and made connections with Anishinaabe Elders and other north shore Lake Huron community members.

Date: $\quad$ October 25, 2012

Place: Shingwauk Residential Schools Centre (SRSC), Algoma University, Sault Ste. Marie, Ontario 
Fieldwork:

Together with Don Jackson (former Director) and Krista McKracken (Archivist), shared information and discussed ideas for further Residential Schools Map development in the broader Atlas-making context.

Date: $\quad$ November 1,2012

Place: $\quad$ Adults in Motion (Garden River First Nation Education Unit), Gitigaan-ziibing (Garden River First Nation)

Fieldwork: $\quad$ At this second outreach meeting, Joanne Thiessen (Co-op Coordinator) and I discussed potential Gitigaan-ziibing (Garden River First Nation) map ideas in more detail in conjunction with constructing a preliminary outline of tasks for the co-op student.

Date:

November 21, 2012

Place:

Adults in Motion (Garden River First Nation Education Unit), Gitigaan-ziibing (Garden River First Nation)

Fieldwork: Stephanie Pyne, Joanne Thiessen, Irene Gray and the potential high school co-op student began initial mapspecific design planning, including a preliminary work plan.

Date:

November 29, 2012

Place:

Wewebijiwang (Little Current), Manitoulin Island, Ontario

Fieldwork: $\quad$ Following up on our first meeting in Regina at the Canadian Association of Geographers AGM conference, I provided an overview of the evolution of the Atlas project and Sophie Edwards described her doctoral research. An introductory discussion commenced concerning the collaborative construction of a potential map in the Atlas based on Sophie's research.

Date: $\quad$ November 30, 2012

Place: Wikwemikong Unceded Indian Reserve, Manitoulin Island, Ontario

Fieldwork: $\quad$ I provided an overview of the evolution of the Atlas project and Naomi Recollet described several community-oriented historical research initiatives. Some preliminary discussions 
Date:

Place:

Fieldwork:

Date:

Place:

Fieldwork:

Date:

Place:

Fieldwork:

Date:

Place:

Fieldwork: about potential map projects involving the Wikwemikong Archives were initiated.

November 30, 2012

Manitowaning, Manitoulin Island, Ontario

Brief discussion with Joe Osawabine (Artistic Director Theatre) and Bruce Naokwegijig (Artistic Director - School) following up on initial discussions with Joahnna Berti (Arts Animator) during phase II of the Atlas project. I provided an overview of the evolution of the Atlas project, including some initial ideas for map-making collaborations with Debajehmujig. Joe and Bruce Naokwegijig described some of their ongoing initiatives, and they agreed to meet again to discuss further collaboration.

December 1, 2012

M'Chigeeng, Manitoulin Island, Ontario

Follow-up on Alan Corbiere's presentation on Anishinaabeg of the Lake Huron Treaty Region's participation in the War of 1812 at the Four Wars of 1812 event in Ottawa on October 12,2012 . I gave Alan a copy of the video I made of the event and we discussed the evolution of the Atlas project and the potential for including geonarratives in Anishnaabemowin.

December 1, 2012

Silver Water, Manitoulin Island, Ontario

I introduced Joseph and Elizabeth Laford to the Atlas and the outreach project ideas. Joe shared stories related to culture, spirituality, language and Anishinaabe community history that could potentially be included in the Atlas. Former Chief of nearby Sheshegwaning First Nation, Elizabeth Laford, shared stories from her past.

December 2, 2012

Wewebijiwang (Little Current), Manitoulin Island, Ontario

More detailed discussions with Sophie Edwards concerning the collaborative development of a geonarrative linked to Sophie's PhD research on the historical figure Anna Jameson 
who travelled throughout the Lake Huron Treaty region in the 1830's and recorded her experiences.

Date:

Place:

Fieldwork:

Date:

Place:

Fieldwork:

Date:

Place:

Fieldwork:
December 13, 2012

Shingwauk Residential Schools Centre (SRSC), Algoma University, Bawating (Sault Ste. Marie, Ontario)

While collaborative work had already begun with the SRSC under the previous directorship of Don Jackson, this was the first time that newly appointed Director, Jonathan Dewar was provided with a comprehensive overview of the evolution of the collaborative Atlas-making project. During the same meeting, Stephanie Pyne and Jonathan Dewar discussed issues related to future collaborations, especially in relation to the Residential Schools Map and E.F. Wilson Biography Map.

In addition, I accompanied the Adults in Motion high school potential co-op student to the SRSC archives for an archival research orientation session with SRSC archivist Krista McCracken.

December 19, 2012

Concordia University, Montreal, Quebec

Together with Dr. Sebastien Caquard (SSRCH Outreach Grant co-applicant), Tom McGurk (MA Student Research Assistant), I provided a comprehensive overview of the evolution of the collaborative Atlas-making project for Algonquin artist, Nadia Myre. This was followed by a group discussion concerning the design and development of a concept-style map to be made in collaboration with Nadia based on her Scar Project work.

December 20, 2012

Carleton University, Ottawa

I met with Dr. Glenn Brauen and Amos Hayes (GCRC team members working on the technological dimension of Atlas design and development) to discuss issues related to the conversion of the Atlas to the Couch DB atlas software framework. 
Date: January $1-18,2013$

Place: $\quad$ Ottawa, Ontario

Fieldwork: Several meetings to discuss issues related to Atlas conversion the Couch DB atlas software framework.

Date:

January 3, 2013

Place:

Victoria Island, Ottawa

Fieldwork:

Video documented Radio Interview with Jacob Wawatie.

Date:

January 11, 2013

Place:

Victoria Island / Parliament Hill

Fieldwork:

Video documented Idle no More Global Day of Action March and Public Rally, and discussed Atlas project with various individuals.

Date:

January 28, 2013

Place:

Serpent River First Nation

Fieldwork: $\quad$ Video-documented Serpent River Youth Information Rally held at the Serpent River Trading Post. Made connections with youth, community Elder, Art Meawasige and others. Jason Pyne, volunteer photographer, also participated.

Date:

February 12, 2013

Place:

Serpent River First Nation

Fieldwork: $\quad$ Provided the Steve and Donna Meawasige with an overview of the Atlas; shared stories and began introductory design discussions for a biography map Introduction to the Lake Huron Treaty Atlas.

Date:

February 12, 2013

Place:

4001 Berri, Studio 5, Montreal, Quebec 
Fieldwork:

Initial meeting between Tom McGurk (research assistant) and Nadia Myre (artist) to discuss mapping options for the Scar Project.

Date:

Place:

Fieldwork:

Date:

Place:

Fieldwork:

Date:

Place:

Fieldwork:

Date:

Place:

Fieldwork:

Date:

Place:

Fieldwork:
February 15, 2013

Kenjgewin Teg Educational Institute, M'Chigeeng First Nation, Ontario

Video documented presentations at public teach-in, discussed Atlas project with individuals.

February 24, 2013

Serpent River First Nation and Bawating (Sault Ste. Marie)

In a phone conversation, provided overview of Atlas and discussed initial map design idea with Joanne Robertson for the potential Four Directions Water Walkers Map.

February 27, 2013

Serpent River First Nation and Bawating (Sault Ste. Marie)

Residential Schools Maps development discussion with Don Jackson.

March 1, 2013

2013 Concordia University EV Building, Montreal, Quebec

Research assistant, Tom McGurk demonstrated proof of concept images for artist Nadia Myre in addition to map design and development discussions.

March 16 - March 232013

Serpent River First Nation

Photographed, video recorded and participated in activities at the Maple Sugar Camp. Volunteer photographer, Jason Pyne, also participated. 
Date:

Place:

Fieldwork:

Date:

Place:

Fieldwork:

Date:

Place:

Fieldwork:

Date:

Place:

Fieldwork:

Place:

Fieldwork:
March 20, 2013

Serpent River First Nation and Wewebijiwang (Little Current)

Introduced Heather Thoma (4Elements Living Arts) to Atlas project and began introductory discussion concerning map ideas for 4Elements Living Arts.

March 26, 2013

Wewebijiwang (Little Current)

Follow-up with Heather Thoma and Sophie Edwards regarding map design and development ideas. Also discussed possibilities for Sophie Edwards to construct an Anna Jameson biography map based on her PhD thesis research.

April 17, 2013

Ottawa, Ontario

Met with Algonquin artist Nadia Myre to discuss progress to date and longer range plans for Scar project map development.

April 19, 2013

Ottawa, Ontario

Initial discussions with Trina Bolam (Aboriginal Healing Fund or AHF), Kathy Kettler (Assembly of First Nations or AFN) and myself, concerning future collaborations around the Residential Schools Map.

April 26, 2013

Ottawa, Ontario

Second discussion with Trina Bolam (AHF), Kathy Kettler (AFN) and myself, concerning future collaborations around the Residential Schools Map. 
Date:

Place:

Fieldwork:

Date:

Place:

Fieldwork:

Date:

Place:

Fieldwork:

Date:

Place:

Fieldwork:

Date:

Place:

Fieldwork:

Date:

Place:
May 9, 2013

Ottawa, Ontario

Discussions with Trina Bolam (AHF), Glenn Brauen, representatives from Tristan Interactive and myself concerning collaborations around the Residential Schools Map.

May 23, 2013

Ottawa, Ontario

Glenn Brauen and I discussed details related to Atlas content and technology development.

June 8-16, 2013

Manitoulin Island (various locations, including Wewebijiwang (Little Current), M'Chigeeng and Silver Water)

Discussed ongoing ideas related to Atlas development and relationship to $\mathrm{PhD}$ thesis.

June 17, 2013

Serpent River First Nation Territory

Walked up part of Orville Commanda's trail with Orville and Blaine Commanda, gathered media, and discussed plans for future trail map.

July 19, 2013

Ottawa, Ontario

Met with Trina Bolam (AHF) to discuss ongoing collaborations related to the Residential Schools Map.

July 26, 2013

Ottawa, Ontario 
Fieldwork: $\quad$ Met with Trina Bolam (AHF) met to discuss ongoing collaborations related to the Residential Schools Map.

Date:

July 27, 2013- August 25

Place:

Gjoa Haven, Nunavut

Fieldwork:

Discussed the Lake Huron Treaty Atlas project with some of the participants at the Gjoa Haven Elder-youth land camp and possibilities for future related mapping projects linked to education, culture and language.

Date:

September 3, 2013

Place:

Ottawa, Ontario

Fieldwork:

Stephanie Pyne and Patricia Kennedy discussed ongoing ideas related to the Governance Paper Trail Map.

Date:

September 9, 2013

Place:

Ottawa, Ontario

Fieldwork:

Glenn Brauen and I discussed ongoing ideas and issues at the intersection of content and technology related to Atlas design and development. 


\title{
Appendix D
}

\author{
Selected Media
}

\section{Selected Digital Media Samples Referenced in Thesis}

Clip

File

Page

Clip 1. 1. Guy Dumas Presentation Audio

Clip 2. 2. Dokis First Nation River Trip Slideshow

Clip 3.

3. Art Petahtegoose Video Clip

Clip 4.

4. Archie McGregor Audio - Part 1

162

Clip 5.

5. Archie McGregor Audio - Part 2

162

Clip 6.

6. Perry Bebaamaash Audio - Part 1

164

Clip 7.

7. Perry Bebaamaash Audio - Part 2

Clip 8.

8. Blaine Belleau Video Clip

Clip 9.

9. Four Wars of 1812 Sample Audio

Clip 10.

10. Gitigaan-ziibing Slideshow

215

Clip 11.

11. Lewis Debassige Atlas Launch Video Clip

Clip 12.

12. Shebeshekong Bay Slideshow

292

Clip 13. 13. Teddy Syrette Reading 1849 Petition - Part 2

291

Clip 14. 14. Teddy Syrette Reading 1849 Petition - Part 2

291

Clip 15.

15. Crystal Migwans on Anna Jameson Video

296

Clip 16. 16. Helicopter ride over Bawating

295 


\section{Selected Presentations Media and Documents Folder}

$\begin{array}{llc}\text { Name } & \text { Subfolder Name } & \text { Page } \\ \text { Presentation 1. } & \text { 1. Memory as Medium - March 2008 } & 290 \\ \text { Presentation 2. } & \text { 2. CAG Quebec2008 - June } & 290 \\ \text { Presentation 3. } & \text { 3. GeoTec2008 - August 2008 } & 290 \\ \text { Presentation 4. } & \begin{array}{l}\text { 4. Circle of All Nations Workshop } \\ \text { May 2009 }\end{array} & 290 \\ \text { Presentation 5. } & \text { 5. OCF M'Chigeeng - October 2009 } \\ \text { Presentation 6. } & \text { 6. ICC Chile2009 - November } & 290 \\ \text { Presentation 7. } & \text { 7. CAGRegina2010 - June } & 290 \\ \text { Presentation 8. } & \text { 8. ICC France2011 - July } & 291\end{array}$




\section{Appendix E}

\section{Thesis-related Publications}

Pyne, S. (2006). Full Responsible Reason and Good Development. Master's Thesis, unpublished, Carleton University.

Pyne, S. (2007). The Political Economy of Relational Space, unpublished coursework paper, Carleton University.

Pyne, S. (2008). Outline for a Postfoundational Rendering of the Lake Huron Treaty Relationship Process, unpublished Ph.D. comprehensive paper, Carleton University.

Pyne, S. (2009). A Cybercartographic Case Study of Lake Huron Treaty Relationships: The Treaties Module as a Reconciliation Tool, unpublished Ph.D. Thesis Proposal, Carleton University.

Caquard, S., Pyne, S., Igloliorte, H., Mierins, K., Hayes, A. and Taylor, D. R. F. (2008). A"Living" Cybercartographic Atlas of Indigenous Perspectives and Knowledge of the GreatLakes Region, Cartographica, 44 (2), 83-100).

Taylor, D.R.F. and Pyne, S. (2010). The History and Development of the Theory and Practice of Cybercartography. International Journal of Digital Earth, 3(1), 1-14.

Wawatie, J. and Pyne, S. (2010). Tracking in Pursuit of Knowledge. In Nathan Kowalsky (Ed.), Hunting - Philosophy for Everyone: In Search of the Wildlife, Blackwell: West Sussex, U.K., Chapter 8.

Brauen, G., Pyne, S., Hayes, A., Fiset, J.P. and Taylor, D.R.F. (2011). Encouraging Transdisciplinary Participation using an Open Source Cybercartographic Toolkit: The Atlas of the Lake Huron Treaty Relationship Process, Geomatica, 65(1), 27-45.

Debassige, L. and Pyne, S. (2012). Devilotion/Devolution: Profits from the Wilderness. In Alan Corbiere, Deborah McGregor and Crystal Migwans (Eds.), Anishinaabewin Niizh: Culture Movements, Critical Moments, M'Chigeeng First Nation: Ojibwe Cultural Foundation, Chapter 1.

Pyne, S. (2012). Spatializing History: The Cybercartographic Atlas of the Lake Huron Treaty Process, in Karl Hele (Ed.), This is Indian Land: The Robinson Huron Treaties of 1850 (in press).

Pyne, S. And Taylor, D.R.F. (2012). Mapping Indigenous Perspectives in the Making of the Cybercartographic Atlas of the Lake Huron Treaty Relationship Process: A Performative Approach in a Reconciliation Context. Cartographica, Special Issue on Indigenous Cartographies and Counter-mapping, 47(2), 92-104 
Pyne, S. (2013a). The Role of Experience in the Iterative Development of the Lake Huron Treaty Atlas. In Taylor, D. R. F. (Ed.), Developments in the Theory and Practice of Cybercartography: Applications and Indigenous Mapping, Amsterdam: Elsevier (in press).

Pyne, S. (2013b). Sound of the Drum, Energy of the Dance: Making the Lake Huron Treaty Atlas the Anishinaabe Way. Unpublished PhD Dissertation, Carleton University. 